\title{
INVESTIGATION ON THE PHYSIOLOGICAL AND PATHOLOGICAL ASPECTS OF THE PROLINE-RICH REGION OF THE MICROTUBULE-ASSOCIATED PROTEIN TAU
}

\author{
Dissertation \\ to acquire the doctoral degree in mathematics and natural science \\ 'Doctor rerum naturalium' \\ At the Georg-August-Universität Göttingen
}

In the doctoral degree program of Biology

At the Georg-August University School of Science (GAUSS)

Submitted by

Adriana Savastano

From Rome, Italy

Göttingen, 2019 


\section{Thesis Committee}

Prof. Dr. Markus Zweckstetter

Structure Determination of Proteins Using NMR, Max-Planck-Institute for Biophysical Chemistry, Göttingen

Translational Structural Biology in Dementia, German Center for Neurodegenerative Disease, Göttingen

Prof. Dr. Tiago Outeiro

Department of Experimental Neurodegeneration, University Medical Center (UMG), Göttingen

Prof. Dr. Kai Tittmann

Department of Molecular Enzymology, Center of Molecular Biosciences, Göttingen

Department of Structural Dynamics, Max-Planck-Institute for Biophysical

Chemistry, Göttingen

\section{Members of the examination board}

Referee: Prof. Dr. Markus Zweckstetter

Co-referee: Prof. Dr. Tiago Outeiro

\section{Other Members of the examination board}

Prof. Dr. Thomas A. Bayer

Division of Molecular Psychiatry, University Medical Center (UMG), Göttingen

Ph.D. Loren B.Andreas

Structure Determination of Proteins Using NMR, Max-Planck-Institute for Biophysical Chemistry, Göttingen

Prof. Dr. Stefan Jakobs, Department of NanoBiophotonics, Max-Planck-Institute for Biophysical Chemistry, Göttingen

Clinic for Neurology, University Medical Center (UMG), Göttingen

Prof. Dr. Johannes Söding,

Computational biology and bioinformatics, Max-Planck-Institute for Biophysical Chemistry, Göttingen

Date of the oral examination: December 13, 2019 


\section{Affidativ}

I hereby declare that the doctoral thesis entitles "Investigation on the physiological and pathological aspects of the proline-rich region of the microtubule-associated protein tau" has been written independently, with no other sources and aids than quoted.

Adriana Savastano

Göttingen, October 2019 


\section{Abstract}

The microtubule-associated protein tau is involved in several neurodegenerative diseases including Alzheimer's disease (AD), Pick's disease (PiD), Progressive Supranuclear Palsy (PSP) and others. The aggregation and fibrillization of hyperphosphorylated tau are considered disease-causing agents in these diseases, which are therefore termed tauopathies.

A major physiological activity of tau is its interaction with microtubules and the regulation of their dynamic rearrangement. Tau phosphorylation regulates its affinity to microtubules and is linked to pathological conditions when aberrant. Phosphorylation is mediated by several kinases and occurs in the proline-rich region as well as in the pseudorepeat domain of the tau sequence. In cryo-electron microscopy studies of tau fibrils purified from patients with $A D$ and $\mathrm{PiD}$, as well as in vitro studies of tau bound to microtubules, structural information regarding the pseudo-repeats was obtained. In contrast, little is known about the structural properties of the proline-rich region when tau is bound to microtubules or aggregated into amyloid fibrils. In this work both physiological and pathological aspects of the structure of tau have been addressed, with a specific focus on the proline-rich region sequence of tau.

In the first project reported in this work, solid-state nuclear magnetic resonance (ssNMR) was used to investigate the contribution of the proline-rich region to the structure of tau fibrils. In vitro fibrils obtained from the tau construct K32, comprising the $\mathrm{P} 2$ domain and the $\mathrm{R} 1, \mathrm{R} 2, \mathrm{R} 3, \mathrm{R} 4$ and $\mathrm{R}^{\prime}$ domains of the pseudo-repeat region, and from two simplistic models, the peptides P2R2 and P2R3, were studied using a combination of ${ }^{13} \mathrm{C}-{ }^{13} \mathrm{C}$ correlation experiments and INEPT-based transfer experiments, which probe the flexible regions of the fibrils. The use of simplistic models, such as the peptides P2R2 and P2R3, improved the quality of the ssNMR spectra and facilitated the identification of residues partially recruited into the core of the fibrils. The analysis of the ssNMR spectra indicated a partial recruitment of the P2 domain within the fibrils, especially of the most hydrophobic patch of the domain, i.e. the ${ }^{225} \mathrm{KVAVVRT}^{231}$ sequence. In the INEPT spectra,the absence of cross peaks from this region suggested a loss of flexibility, most 
likely due to the formation of hydrophobic interactions between the ${ }^{225} \mathrm{KVAVVRT}^{231}$ sequence and the hexapeptides in the R2 and the R3 domains.

Liquid-liquid phase separation (LLPS) of intrinsically disordered proteins (IDPs) into compartments without surrounding membranes is implicated in the regulation of biological processes. However, little is known regarding the molecular mechanisms that influence biological processes in condensed phases. In the second project, I therefore studied LLPS of tau and its connection to the polymerization of tubulin into microtubules. The studies showed that LLPS of tau, its phosphorylation, and conformational changes upon binding to microtubules are functionally linked. Tau phosphorylated at diseaseassociated epitopes condensed into liquid-like compartments, tubulin partitioned into these drops, but it was unable to grow into microtubules. The functional link between LLPS and tubulin polymerization was provided by a conformational change in the prolinerich region of tau upon binding to tubulin. Phosphorylation blocks the functionally required conformational change through formation of salt bridges between phosphate groups and conserved arginine residues in the proline-rich region of tau. The data established a mechanistic framework in which LLPS and conformational changes in tau cooperate to drive formation of cytoskeletal tracks. 


\section{Acknowledgements}

First and foremost I would like to thank Prof. Dr. Markus Zweckstetter for his supervision and guidance during these years. Thank to him I had the opportunity to work on many exciting, sometimes challenging, projects, in an amazingly inspiring scientific environment.

I would also like to thank Prof. Dr. Tiago Outeiro and Prof. Dr. Kai Tittman for being part of my thesis committee and always giving interesting points of discussion during our meetings.

I would like to thank Ph.D Loren Andreas and Dr. Riza Dervisoglu for their assistance and support during the acquisition of the ssNMR experiments presented in this work.

Many thanks go to Kerstin Overkamp, for the synthesis of the peptides used in this work, and to Gudrun Heim for the EM picture of the fibrils samples.

My deepest gratitude goes to Dr. Filippo Favretto for rescuing at my very, goofy, beginning in the lab and teaching me literally all that he knows. Thank you for never stepping back when I needed you and always being there, eager to help unconditionally. You have been such an amazing friend, colleague and a role model to aim to.

During these four years, our laboratory went through a complete rearrangement of its members. I have found my self in the middle of these changings and had therefore the possibility to meet many, amazing people. I would like to thank the "old" members of the AK Zweckstetter group Dr. Garima Jaipuria, Dr. Javier Oroz, Dr. Harindranath Kadavath, Dr. Yunior Cabrales, Dr. Aldo Camacho, Dr. Susmitha Ambadipudi and Dr. Luis Fonseca. Thank you all, for welcoming me in the lab and supporting me at the beginning of my PhD.

To the members and friends of the "new" AK Zweckstetter I owe much gratitude for representing a sort of small family and creating an amazing working environment. I would like to thank Antonia, Marjia, Timo, Conny, Sol, Alain, Tae-kyung, Hessam, Pjiush, David, Glwadys, Matthew, Gunnar and Tina. A big thank goes to my amazing officemates, Maria and Crhistian, for creating such a cheerful atmosphere. 
I would like to thank the friends and colleagues at the NMR || department at the MaxPlanck-Institute for Biophysical chemistry Ezster, Cecily, Kumar, Leif, Sebastian, Mookyong, Supriya, Riza, Marcel not only for the help in front of the spectrometers but also for the "small" and the "not so small" talks in the coffe room.

A mia madre e mio padre devo molto. Grazie per aver camminato con me lungo questo tortuoso percorso. A volte é stato difficile, cosí difficile da non trovare nemmeno le parole per descrivere come mi sentissi. Grazie per aver creduto, forse anche piú di me, che sarei riuscita a portare a termine questo progetto e per avermi insegnato i valori che fanno di me la persona che sono oggi. Lucio, grazie per le lunghe telefonate, gli sfoghi e le interminabili conversazioni che mi hanno dato sicurezza e forza nelle giornate piú pesanti. Al mio speciale Nonno Yoda, grazie per avermi seguito da lontano e con tanto cuore, per le e-mail che mi hanno tenuto compagnia in questi anni e per non avermi mai fatto sentire sola.

Niels, grazie per essere stato il mio punto di riferimento piú solido. Mi hai spinto a dare il meglio di me e allo stesso tempo mi hai confortato quando ero priva di energia.Grazie per le tante, piccole lezioni di teoria di NMR e di vita.

Surviving to these years would have never been possible without the support of my friends, those in Göttingen and those in Italy. Yagmur, thank you for being the closest, most sincere friend I could find during this important period of my life. Beate, Melanie, Socrates, Meike, Joana and Kerstin thank you the amazing time in Göttingen. I would like to thank Julia and Stephan with whom I shared two amazing years. Nicola, Marsha and Marie you have been an import reference in these years, I knew I could always count on you, for professional and personal issues.

Valeria, Beatrice, Ambra, Michela, Vanessa, Federica and Giulia, thank you for the precious friendship we share and for the life advices you constantly give to me.

I want to express my endless gratitude to the RNA tie Club members, Valentina, Francesco e Alessandro: you guys have been a solid column, I have missed you and I miss you very much, every day. 
Finally, if this time spent in Göttingen have given to me so much, it has also taken a lot back. People came and people left, from the city, from my life. I want to thank all those people I have lost in these years for teaching me great lessons of life. I am just grateful I have met all of you, whether it was for a mere second, for a month or for some years. Thank you. 


\section{Table of contents}

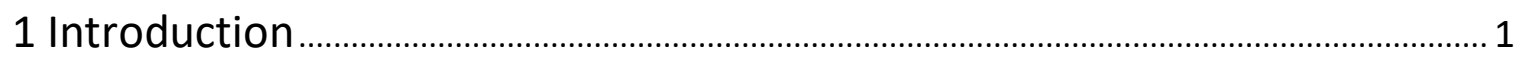

1.1 NEURODEGENERATION AND NEURODEGENERATIVE DISEASES........................................

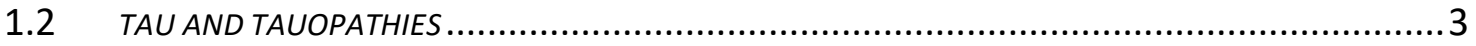

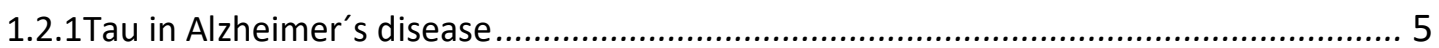

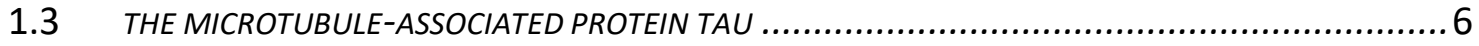

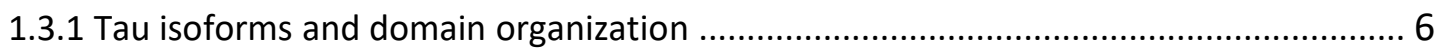

1.3.2 The intrinsically disordered properties of Tau ............................................................. 9

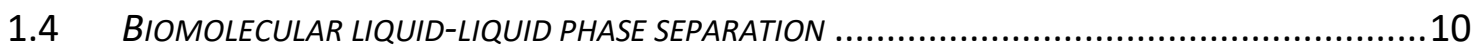

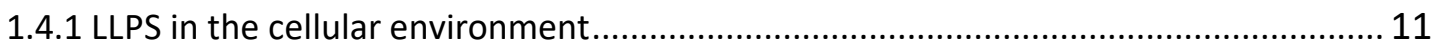

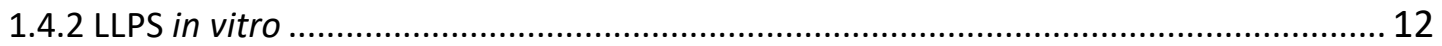

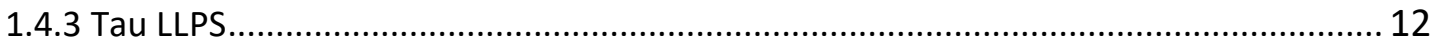

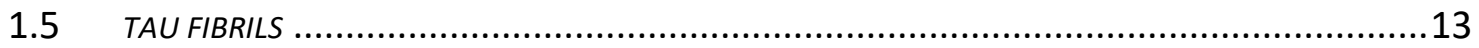

1.5.1 Amyloid fibrils and their polymorphism .............................................................. 14

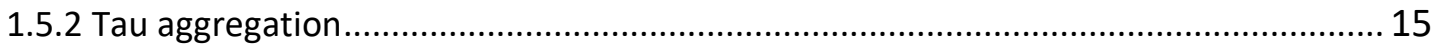

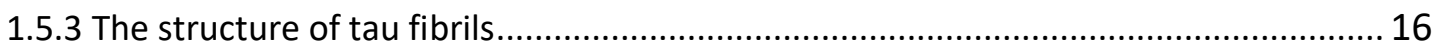

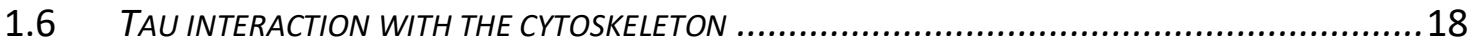

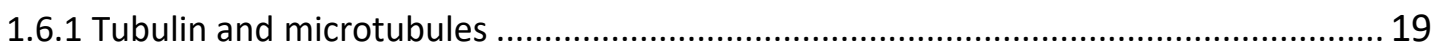

1.6.2 Tau regions involved in the interaction with tubulin and microtubules ......................20

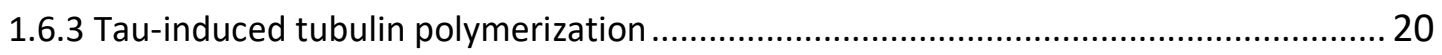

1.6.4 The role of charges in tau-tubulin/microtubule interaction........................................ 21

1.6.5 Structural insights into the binding of tau to tubulin and microtubules ...................... 22

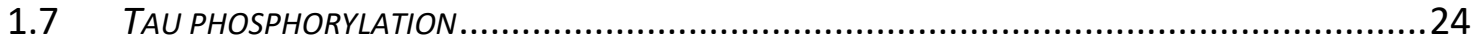

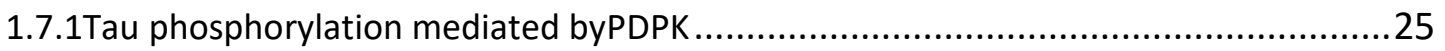

1.7.2 Tau phosphorylation mediated by non-PDPKs ......................................................... 26

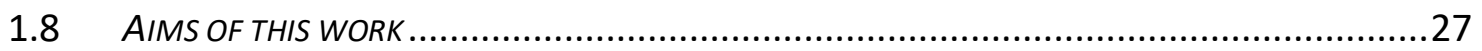

2 Materials and methods ..................................................................................................2

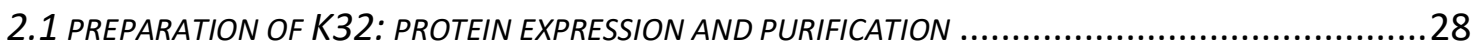

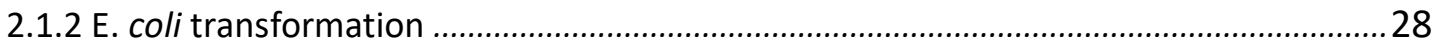




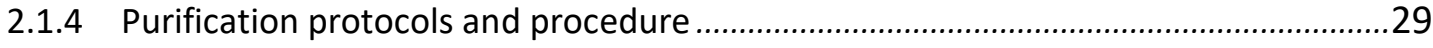

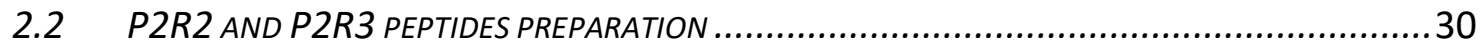

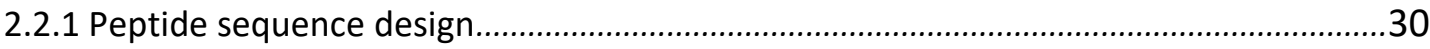

2.2.2 PCR

2.2.3 Restriction enzyme double digestion and ligation reaction ..........................................32

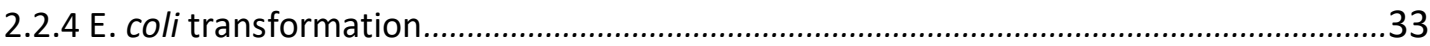

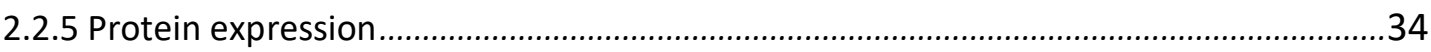

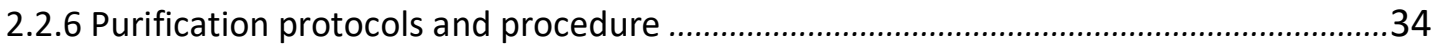

2.3 UNIFORM ISOTOPE LABELING OF PROTEINS FOR SOLUTION AND SOLID-STATE NMR .................35

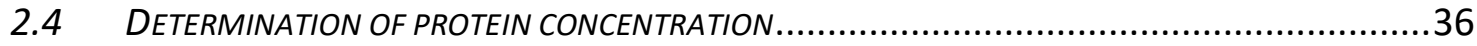

2.5 CIRCULAR DICHROISM OF P2R2 AND P2R3 PEPTIDES IN THE MONOMERIC FORM ..................37

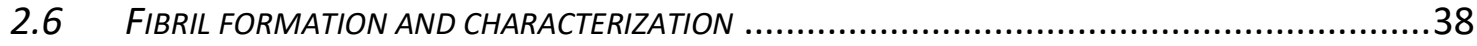

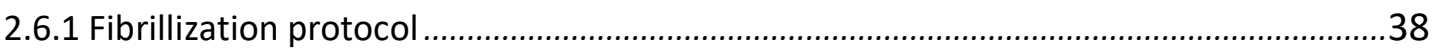

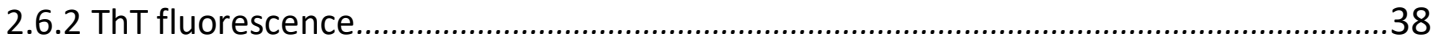

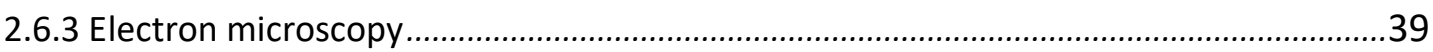

2.6.4 Circular dichroism of fibrils obtained from K32 and the P2R2 and P2R3 peptides.......39

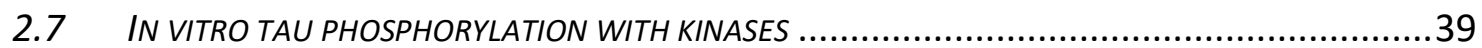

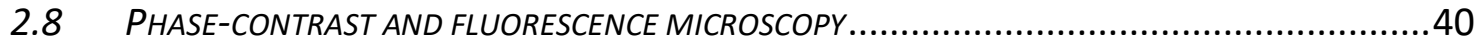

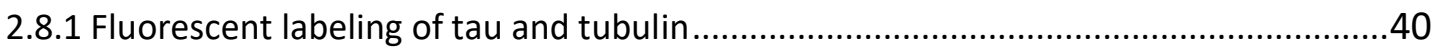

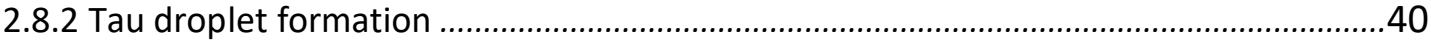

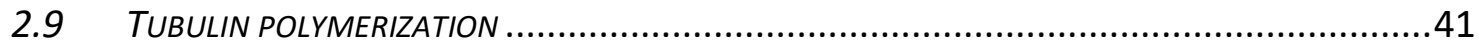

2.9.1 Assemblyof paclitaxel-stabilized microtubules..............................................................

2.9.2 Tubulin polymerization from tau droplets .........................................................................

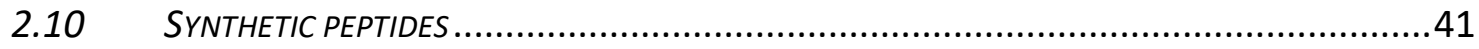

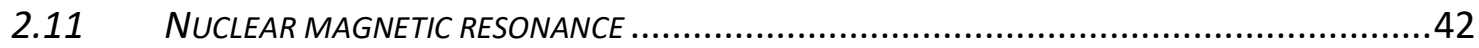

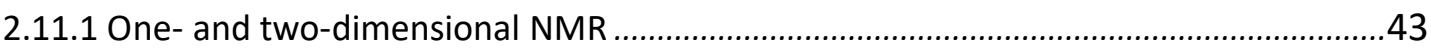

2.11.2 2D heteronuclear single quantum coherence (HSQC) .................................................44

2.11.2.1HSQC experiment settings and data analysis for the project I.......................................45

2.11.2.2HSQC experiment settings and data analysis for the project II.....................................46 
2.11.3.1 TROSY experiment settings and data analysis.........................................................49

2.11.4 3D NMR experiments for resonance assignment ..................................................49

2.11.4.1 3D experiments settings and data analysis.............................................................50

2.11.5 The Nuclear Overhauser Effect ................................................................................ 52

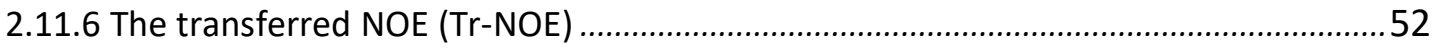

2.11.6.1 2D NOE experiments for the structure determination of Tau(P2) ...............................52

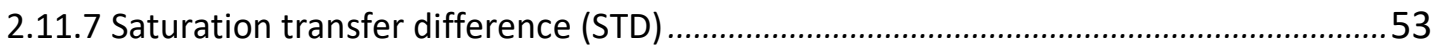

2.11.7.1 STD NMR experiments setting and data analysis...................................................54

2.11.8 Characterization of protein diffusion via NMR ............................................. 57

2.11.8.1 NMR diffusion experiments settings and data analysis..............................................57

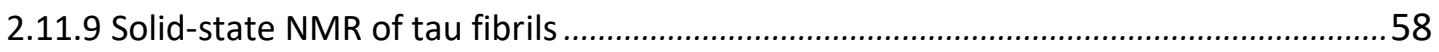

2.11.9.1 Differences between liquid-state and solid-state NMR ..............................................58

2.11.9. ${ }^{13} \mathrm{C}-{ }^{13} \mathrm{C}$ correlation obtained via proton-driven spin diffusion (PDSD)................................59

2.11.9.3 2D INEPT transfer-based ${ }^{1} \mathrm{H}-{ }^{13} \mathrm{C}$ through-bond correlation............................................60

2.11.9.4 Preparation of solid-state NMR samples and experimental settings................................60

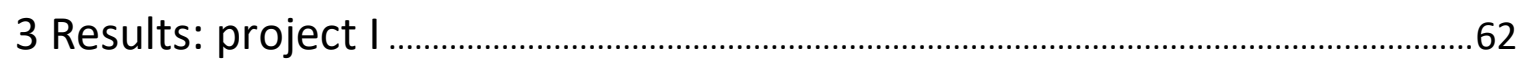

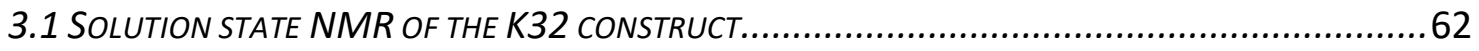

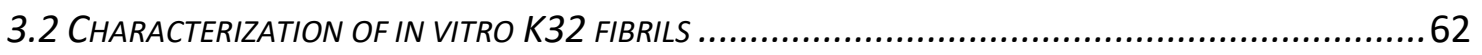

3.3 PROTON DRIVEN SPIN DIFFUSION (PDSD) EXPERIMENT ON K32 FIBRILS ...............................64

3.4 DETECTION OF THE FLEXIBLE REGIONS IN K32 FIBRILS ...........................................67

3.5 PREPARATION OF P2R2 AND P2R3 POLYPEPTIDES FOR A SIMPLISTIC MODEL OF TAU FIBRILS.........70

3.6 CHARACTERIZATION OF THE INTRINSICALLY DISORDERED PROPERTIES OF P2R2 AND P2R3 ...........70

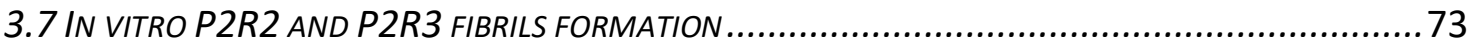

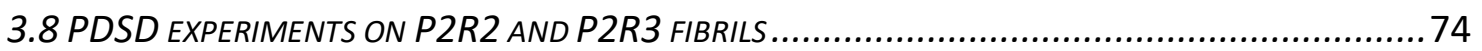

3.9 DETECTION OF THE FLEXIBLE REGIONS IN P2R2 AND P2R3 FIBRILS ................................. 78

3.10 COMPARISON OF THE PDSD SPECTRA OF P2R2, P2R3 AND K32 ...............................83 
4.1 THE RELEVANCE OF THE TAU PROLINE-RICH REGION IN PATHOLOGICAL CONDITIONS ... 84

4.2 STRUCTURAL PROPERTIES OF THE RIGID CORE OF K32 FIBRILS......

4.3 THEFLEXIBLE REGIONS IN K32 FIBRILS. 86

4.4 THE USE OF SIMPLISTIC MODELS FOR THE STUDY OF TAU FIBRILS.

4.5 BIOPHYSICAL CHARACTERIZATION OF P2R2/P2R3 FIBRILS.

4.6 CONTRIBUTION OF THE P2 DOMAIN TO THE CROSS-BSTRUCTURE OF P2R2 AND P2R3 FIBRILS......88

5 Results:project II 91

5.1 TAU INTERACTION WITH MICROTUBULES AT DIFFERENT CONCENTRATIONS AND TEMPERATURES ..... 91

5.2 TAU INTERACTION WITH SOLUBLE TUBULIN .93

5.3 TAU PHOSPHORYLATION WITH CDK2/CYCA2 94

5.4 INTERACTION OF THE PHOSPHORYLATED TAU WITH TUBULIN/MICROTUBULES. 96

5.5 TAU LLPS AND TUBULIN POLYMERIZATION. 98

5.6 IMPACT OF PHOSPHORYLATION OF TAU'S PROLINE-RICH REGION ON LLPS-MEDIATED TUBULIN POLYMERIZATION..... 100

5.7 TAU PHOSPHORYLATION BYMARK2 AND INTERACTION WITH MICROTUBULES 101

5.8IMPACT OF PHOSPHORYLATION OF TAU'S PSEUDO-REPEAT REGION ON LLPS-MEDIATED TUBULIN POLYMERIZATION.. 103

5.9 BINDING OF TAU'S PROLINE-RICH REGION TO SOLUBLE TUBULIN. 105

5.10 STRUCTURAL INSIGHTS INTO THE INTERACTION OF TAU(P2) WITH MICROTUBULES 106

5.11 EFFECT OF TAU(P2) PHOSPHORYLATION AT T231 ON THE INTERACTION WITH TUBULIN/MICROTUBULES 108

5.12 STRUCTURAL CHANGES INDUCED BY THE PHOSPHORYLATION IN THE PROLINE-RICH REGION OF TAU

6 Discussion: project II

6.1 TAU INTERACTION WITH PACLITAXEL-MICROTUBULES AND SOLUBLE TUBULIN.....

6.2 TAU PHOSPHORYLATION IN DIFFERENT REGIONS OF THE SEQUENCE.

6.3 TUBULIN POLYMERIZATION IN TAU PHASE SEPARATION CONDITIONS

6.4 INTERACTION BETWEEN THE PROLINE-RICH REGION OF TAU AND TUBULIN/MICROTUBULES 
7 Bibliography 118

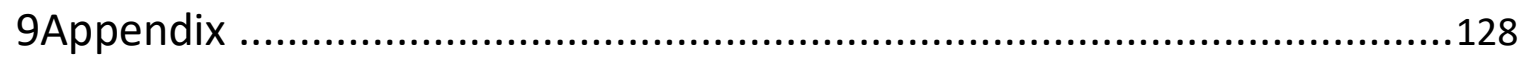

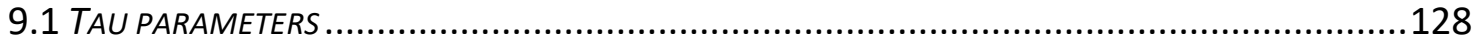

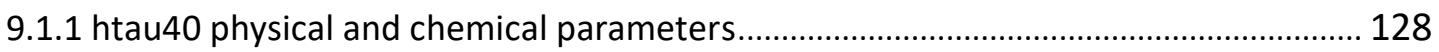

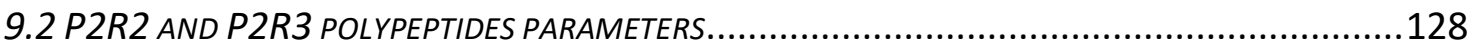

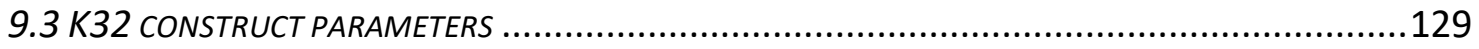

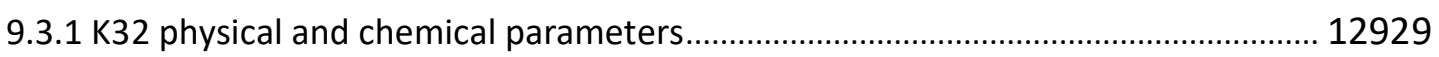

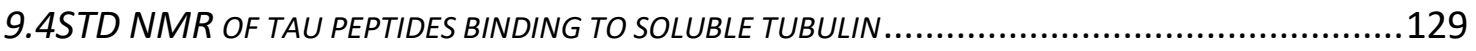

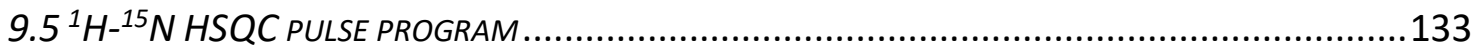

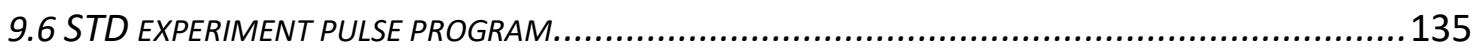

9.7 NMR DIFFUSION PGSTE-WATERGATE PULSE PROGRAM ................................................139

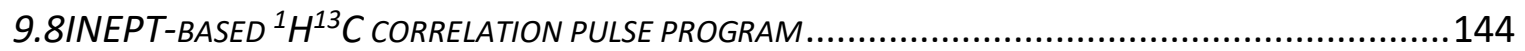




\section{Table of Figures}

Figure 1.1 Brain areas affected by neuronal loss in selected neurodegenerative diseases..

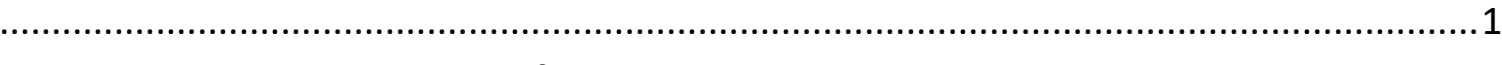

Figure 1.2. Global distribution of neurodegenerative diseases and dementia ..................3

Figure 1.3. Tau in physiological and pathological conditions. ........................................4

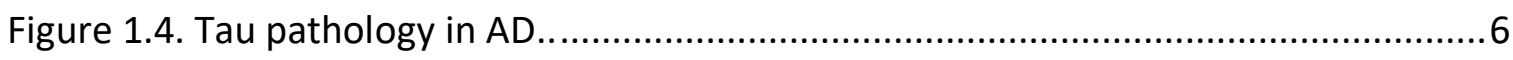

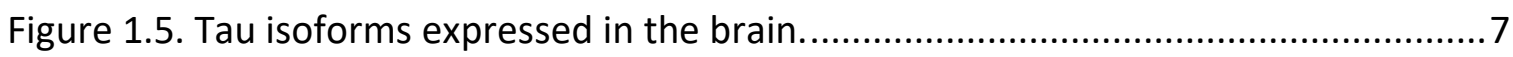

Figure 1.6. Tau sequence regions and their role in tau activity. ..................................... 8

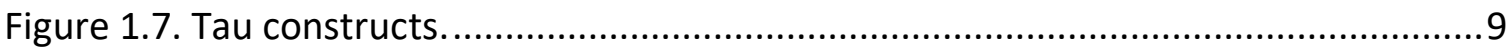

Figure 1.8. Charge distribution in the tau sequence. ..................................................

Figure 1.9. Tau LLPS in physiological and pathological conditions.................................13

Figure 1.10. Cross- $\beta$ structure of amyloid fibrils. ........................................................ 14

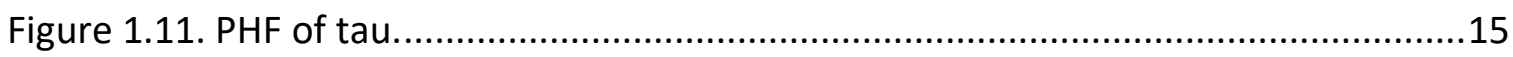

Figure 1.12.Cryo-EM structures of tau fibrils purified from AD and PiD brain..................17

Figure 1.13. Schematic representation of the microtubule filament organization...........19

Figure 1.14. Modelling of tau bound to the microtubule surface...................................23

Figure 1.15. Tau residues phosphorylated by Cdk2, Cdk5/GSK3 $\beta$ and MARK2................24

Figure 2.1. P2R2 and P2R3 domain organization and sequence.................................. 31

Figure 2.2. Schematic representation of the energy level splitting in an external magnetic

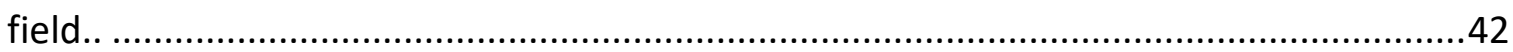

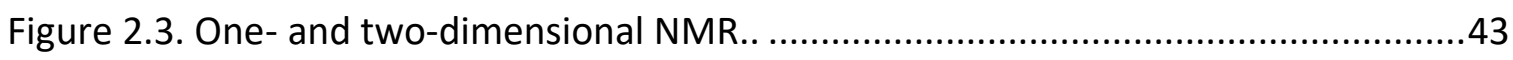

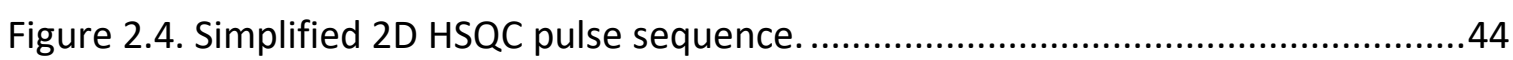

Figure 2.5. Schematic representation of the STD NMR experiment...............................54

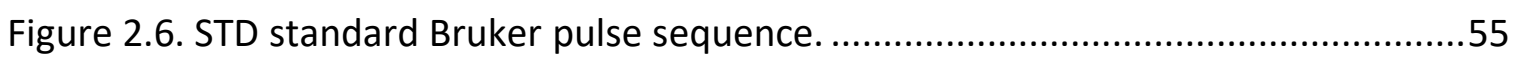

Figure 2.7. Transfer of magnetization in a PDSD experiment. .......................................59

Figure 3.1. Backbone and side chain assignment of $\mathrm{K} 32$ in solution..............................62

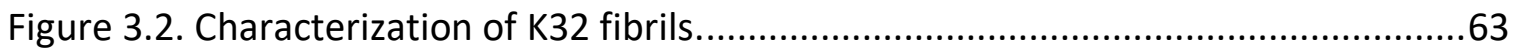

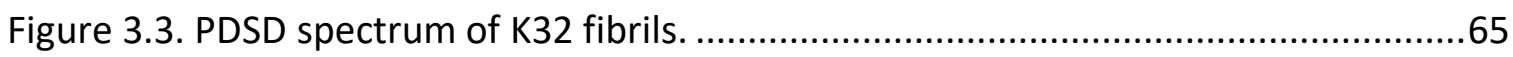

Figure 3.4. Analysis of the PDSD spectrum of K32 fibrils...............................................66

Figure 3.5 Detection of the flexible regions in K32 fibrils............................................68

Figure 3.6. Selected regions of the SSNMR INEPT spectrum of K32..............................69

Figure 3.7 Purification of P2R2 and P2R3 polypeptides by size exclusion chromatography.

Figure 3.8. Intrinsically disordered properties of the P2R2 and P2R3 polypeptides.........71

Figure 3.9. C $\alpha$ chemical shift difference between htau40 and P2R2 or P2R3. ................72

Figure 3.10. Decay of NMR signals of P2R2 and P2R3 in NMR diffusion experiments. ....73

Figure 3.11. Characterization of the in vitro fibrils of P2R2 and P2R3 ..........................74

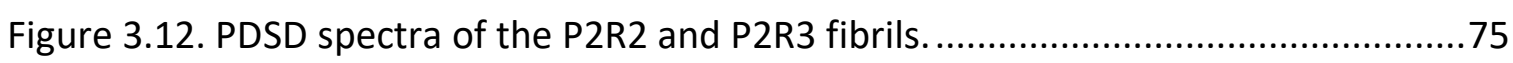

Figure 3.13. The threonine resonance region in the PDSD spectra of P2R2 and P2R3....76 
Figure 3.14. Comparison of the P2R2 and the P2R3 PDSD spectra with a previously reported spectrum of $\mathrm{K} 19$ and resonance assignments of htau40 fibrils. ........................77

Figure 3.15. Detection of the flexible regions in P2R2 fibrils........................................79

Figure 3.16. Detection of the flexible regions in P2R3 fibrils. ...................................... 80

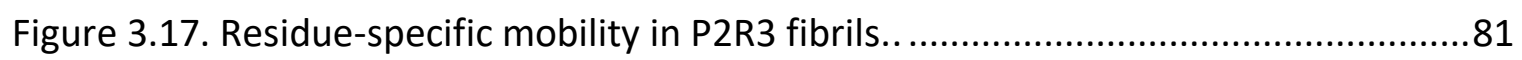

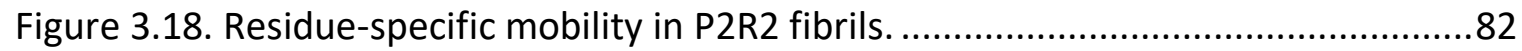

Figure 3.19. Superposition of the threonine region of K19, K32, P2R2 and P2R3 PDSD

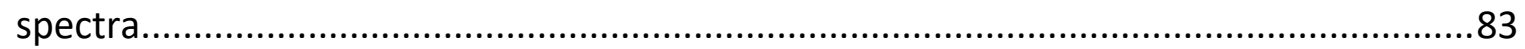

Figure 5.1. NMR profile of tau interacting with paclitaxel-stabilized microtubules at

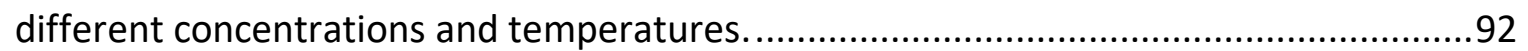

Figure 5.2. NMR profile of htau40 interacting with soluble tubulin. ..............................94

Figure 5.3. Phosphorylation of the proline-rich region of tau by CDK2/CycA2 ................95

Figure 5.4. Quantification of tau phosphorylation mediated by Cdk2/CycA2 .................96

Figure 5.5. Interaction of tau phosphorylated by Cdk2/CycA2 with soluble tubulin. .......97

Figure 5.6. Microtubule-interaction of tau phosphorylated by Cdk2/CycA2...................98

Figure 5.7. Differential interference contrast (DIC) microscopy and fluorescence

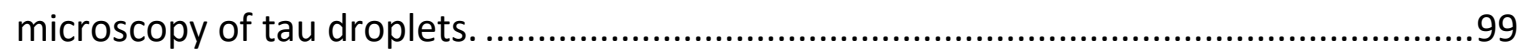

Figure 5.8. Differential interference contrast (DIC) microscopy and fluorescence microscopy of tau droplet-induced tubulin polymerization...........................................99

Figure 5.9 Differential interference contrast (DIC) microscopy and fluorescence microscopy of pTau(Cdk2/CycA2) droplets upon addition of tubulin.............................100

Figure 5.10. Phosphorylation of tau mediated by the kinase MARK2 ...........................102

Figure 5.11. Quantification of tau phosphorylation mediated by MARK2. ......................103

Figure 5.12. Interaction of MARK2-phosphoryated tau with microtubules....................103

Figure 5.13. Differential interference contrast (DIC) microscopy and fluorescence microscopy of pTau(MARK2) droplet-induced tubulin polymerization. ...........................104

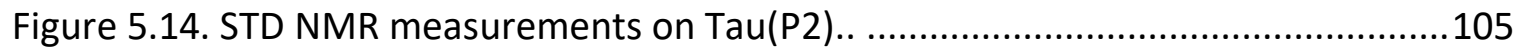

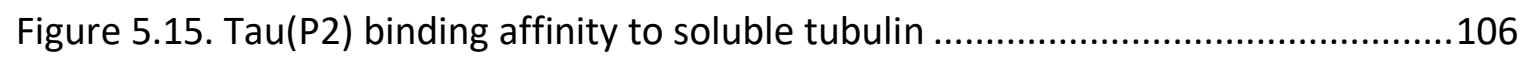

Figure 5.16. Structure of the proline-rich region of Tau bound to MTs. .......................107

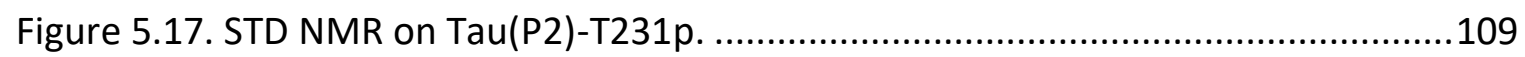

Figure 5.18. Effect of the phosphorylation of T231 on Tau(P2).................................110

Figure 5. 19. Structural impact of tau phosphorylation mediated by Cdk2/CycA2 .........111

Figure 9.1. Tau peptides used for additional STD NMR experiments..............................130

Figure 9.2. Selection of peaks in the 1H 1D STD-NMR spectra for the analysis of the binding affinites.

Figure 9.3. Binding affinities of selected amino acid residues in Tau(P2)short and $\operatorname{Tau}(\mathrm{P} 2-\mathrm{R} 3)$ 


\section{List of Tables}

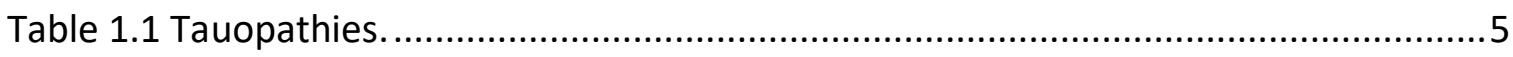
Table 2.1 Gyromagnetic ratios, NMR frequencies (in a $9.4 \mathrm{~T}$ field $=400 \mathrm{MHz}$ ) and natural abundancies of the nuclei commonly observed in protein NMR experiments.

Table 2.2. Parameters for $1 \mathrm{H}-15 \mathrm{~N}$ HSQC and $1 \mathrm{H}-13 \mathrm{C}$ HSQC experiments related to the project I.

Table 2.3 Selected parameters for $1 \mathrm{H}-15 \mathrm{~N}$ HSQC experiments related to the project II .48

Table 2.4. List of 3D experiments recorded on K32, P2R2 and P2R3. .51

Table 2.5. Selected parameters for ssNMR experiments performed on tau fibrils. 61

Table 3.1 Secondary structure content calculated with the Dichroweb software from

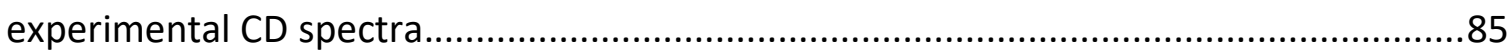

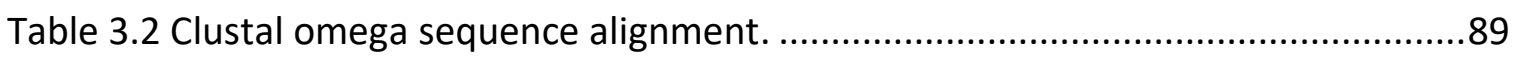

Table 5.1. $K_{D}$ values for selected residues of the proline-rich region.............................106

Table 5.2 Structural statistics for the conformation of Tau(P2) bound to microtubules.

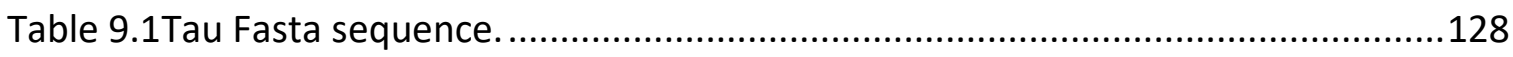

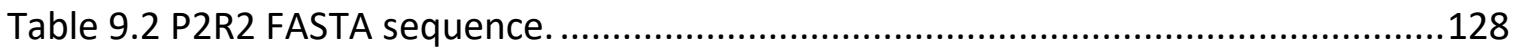

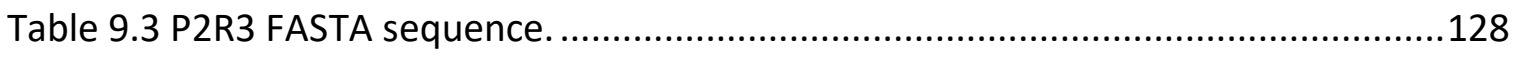

Table 9.4 P2R2 and P2R3 physical and chemical parameters. ....................................129

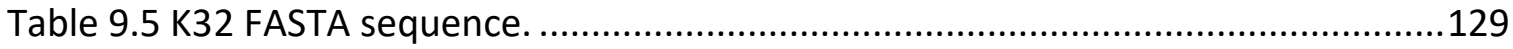

Table 9.6 KD values reported for selected residues located in two different domains of tau. 


\section{Abbreviations}

$A D$

PD

HD

ALS

CNS

NFTs

SPS

MAPS

PiD

NT

PB

PHFs

MTs

PRR

MTBR

IDPs

NMR

LLPS

DIC

PEG

SSNMR

EPR

Cryo-EM

SFS

NPFS

WPFs

HSQC

CSF

MTCO

MARK2

PTM

PDPK

Cdk

GSK

TTBK 1/2

CaMKII

C-abl

CD

ThT

TROSY

INEPT

SW

NS

TD
Alzheimer's disease

Parkinson's disease

Huntington disease

Amyotrophic lateral schlerosis

Central nervous system

Neurofibrillary tangles of tau in $A D$

Senile plaques of tau in $A D$

Microtubule-associated proteins

Pick's disease

Neuropil treads

Pick's bodies

Paired helical filaments of tau in $A D$

Microtubules

Proline-rich region of tau

Microtubule-binding region of tau

Intrinsically disordered proteins

Nuclear magnetic resonance

Liquid-liquid phase separation

Difference interference contrast

Polyethylene glycol

Solid-state NMR

Electron paramagnetic resonance

Cryogenic electron microscopy

Straight filaments of tau in $A D$

Narrow pick filaments of tau in PiD

Wide pick filaments

Heteronuclear single quantum coherence/correlation

Cerebrospinal fluid

Microtubules organizing centers

Microtubule affinity-regulating kinase 2

Post-translational modification

Proline-directed protein kinase

Cyclin dependent kinase

Glycogen synthase kinase

tau-tubulin kinases $1 / 2$

$\mathrm{Ca}^{2+} /$ calmodulin-dependent protein kinase II

Cytoplasmic Abelson leuckemia

Circular dichroism

Thioflavin $T$

Transverse Relaxation-Optimized Spectroscopy

Insensitive Nuclei Enhancement by Polarization Transfer

Spectral widths in NMR experiments

Number of scans in NMR experiments

Time domain in NMR experiments 
Nuclear Overhauser Effect

STD

Saturation Transfer Difference 


\section{Introduction}

\subsection{Neurodegeneration and neurodegenerative diseases}

Neurodegeneration is the pathological process which causes the loss of structure and/or function of neuronal cells and ultimately their death. In neurodegenerative diseases, such as Alzheimer's disease (AD), Parkinson's disease (PD), Huntington disease (HD), amyotrophic lateral sclerosis (ALS) and many others, a specific area of the central nervous system (CNS) is affected by neuronal cell death (Figure 1.1). The neuronal loss is responsible for the progressive decline of cognitive and physical functions of the patients ${ }^{1}$.

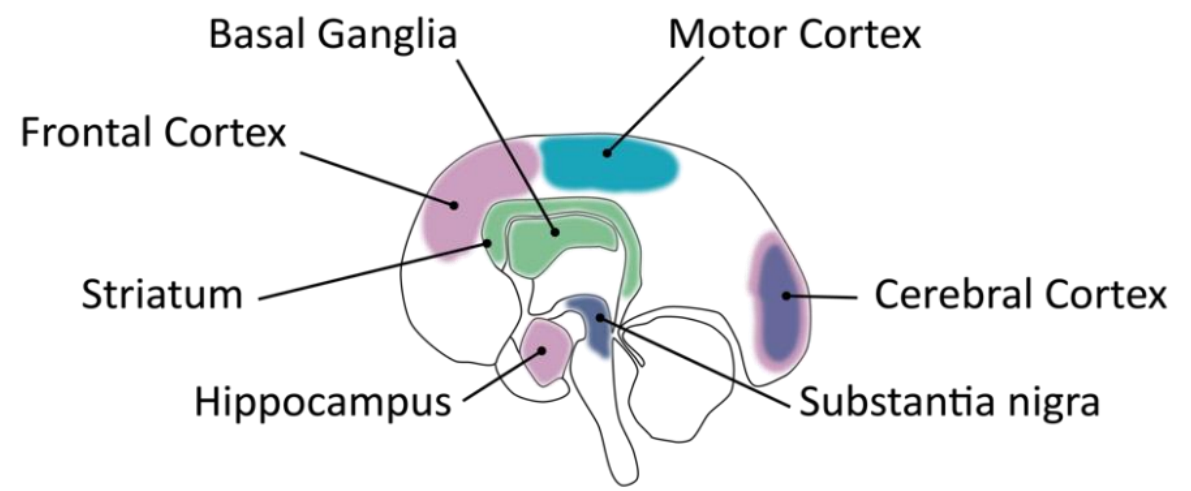

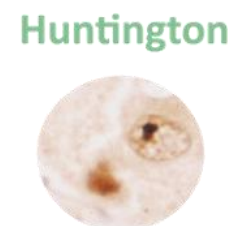

Alzheimer's Disease

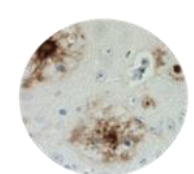

Neurofibrillary tangles

(tau)

Senile plaques

$(A \beta)$

\section{Parkinson's Disease}

Neuronal inclusions

(huntingtin)

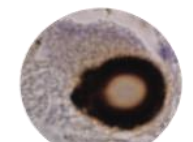

Lewy bodies ( $\alpha$-synuclein)
Amyotrophic Lateral Sclerosis

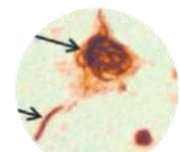

Protein inclusions

(TDP-43)

Protein inclusions

(FUS)

Figure 1.1 Brain areas affected by neuronal loss in selected neurodegenerative diseases. The misfolding and aggregation of specific proteins are considered pivotal events triggering neurodegeneration in different areas of the brain. In AD, accumulation of Neurofibrillary Tangles (NFTs) and Senile Plaques (SPs) is observed in the frontal cortex, cerebral cortex and in the hippocampus (regions marked in pink); these proteinaceous aggregates are mainly constituted of tau and A $\beta$ protein. In ALS, many proteins, including 
FUS and TDP-43, are found in intracellular inclusions in neurons of the motor cortex. Brains from patients affected by HD show accumulation of mutated forms of the huntingtin protein in the striatum and the basal ganglia regions. The substantia nigra and the cerebral cortex are affected in PD, where aggregated forms of $\alpha$-synuclein are found in Lewy bodies. Adapted from Shamsi et al., Int J Biol Macromol, 2017. NFTs taken from Kovacs, Handb Clin Neurol, 2017; SPs taken from Savastano et al., J Alzheimers Dis, 2015; FUS and TDP43 protein inclusion taken from Droppelmann et al., Amyotroph Lateral Scler Frontotemporal Degener, 2014; Lewy bodies taken from Wakabayashi et al., Neuropathology, 2007; Huntingtin inclusion taken from Sieradzan et al., Exp Neurol, 1999.

The etiology of neurodegeneration is still a matter of debate. Many mechanisms have been identified as causing neuronal dysfunction and cell death, including oxidative stress, protein misfolding, mitochondrial dysfunction, DNA damage and many more ${ }^{2,3}$. Protein misfolding, by which a protein loses its normal three-dimensional structure ${ }^{4}$, and aggregation are considered key events in the onset of neurodegenerative diseases ${ }^{5}$. Many neurodegenerative diseases are associated with the misfolding and/or aggregation of specific proteins, e.g. the huntingtin protein (in $\mathrm{HD}^{6}$, the microtubuleassociatedproteintauand the amyloid- $\beta$ peptide (in $A D)^{7}, \alpha$-synuclein (in PD) ${ }^{8}$ (Figure 1.1).For this reason many of them are also described as proteinopathies ${ }^{2}$.

Aging is considered an important risk factor, because at a late stage of life neurons are more susceptible to death ${ }^{3}$. In the twenty-first century, lifespan has increased due to the improved health conditions, with the unintended consequence of increasing the chances for the onset of neurodegenerative diseases. Other risk factors, such as genetic hereditability, obesity, traumatic brain injuries, gender, and many othersmust not be excluded $^{1,9}$. According to the latest Alzheimer's disease report, the economic impact of Alzheimer and other dementias will account for $\$ 290$ billion in $2019^{10}$. This and the big impact on society's health turned neurodegenerative diseases into one of the most stricking illnesses among the human diseases ${ }^{11,12}$. Up to now, there is no cure for neurodegenerative diseases and the current therapies only act as a palliative of the clinical symptoms ${ }^{13}$. For this reason, a deep understanding of the molecular mechanisms involved in the onset of neurodegeneration is necessary. 
a

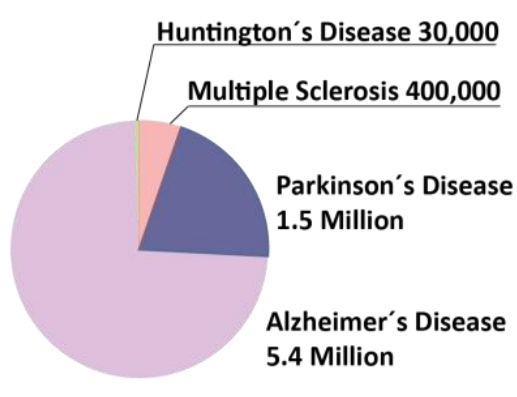

b

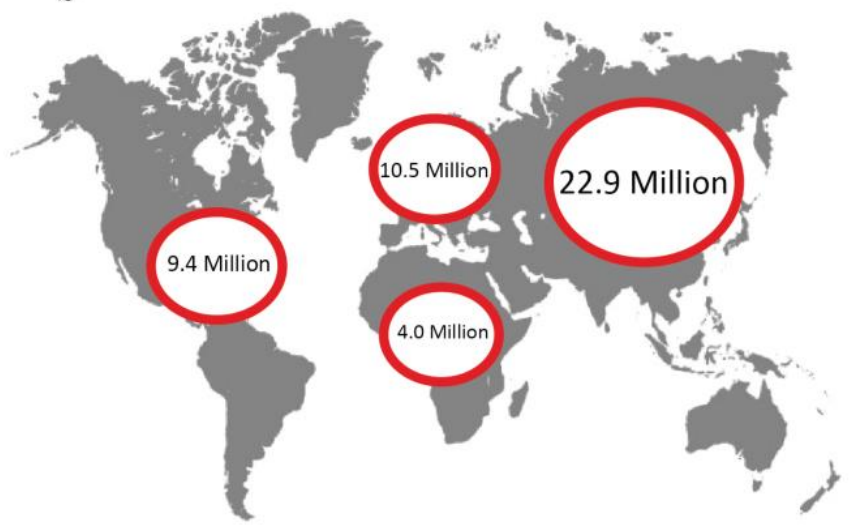

Figure 1.2. Global distribution of neurodegenerative diseases and dementia. A constantly increasing number of people is affected by neurodegenerative disorders. a) In the U.S., AD and PD are the most common neurodegenerative diseases (adapted from Somnath, US pharmacist, 2012 ${ }^{14}$ ). b) All over the world, dementia is having a big impact on life expectancy of the population and it represents a socio-economic burden (adapted from the World Alzheimer's disease report 2018).

\subsection{Tau and Tauopathies}

This work has focused on the study of selected physiological and pathological aspects of the microtubule-associated protein tau, whose intracellular aggregation is a common hallmark of several neurodegenerative diseases, classified as tauopathies ${ }^{15}$.

Tau belongs to the family of microtubule-associated proteins (MAPs) and displays intrisically disordered properties ${ }^{16,17}$. In physiological conditions, the protein is localized in the axons of neuronal cells and interacts with microtubules, assisting their dynamic rearrangements ${ }^{18-21}$. Post-translational modifications regulate tau activities in the cell. The aberrant phosphorylation occurring under pathological circumstances induces tau detachment from microtubules and their disruption ${ }^{22,23}$. As the hyperphosphorylated tau detaches from the microtubules, it further aggregates into fibrils ${ }^{24}$ (Figure 1.3). After its detachment from the microtubules, free tau in the cytoplasm can be mislocated to dendrites, where it interacts with actin. This causes the formation of F-actin bundles and synaptic toxicity ${ }^{25}$. 

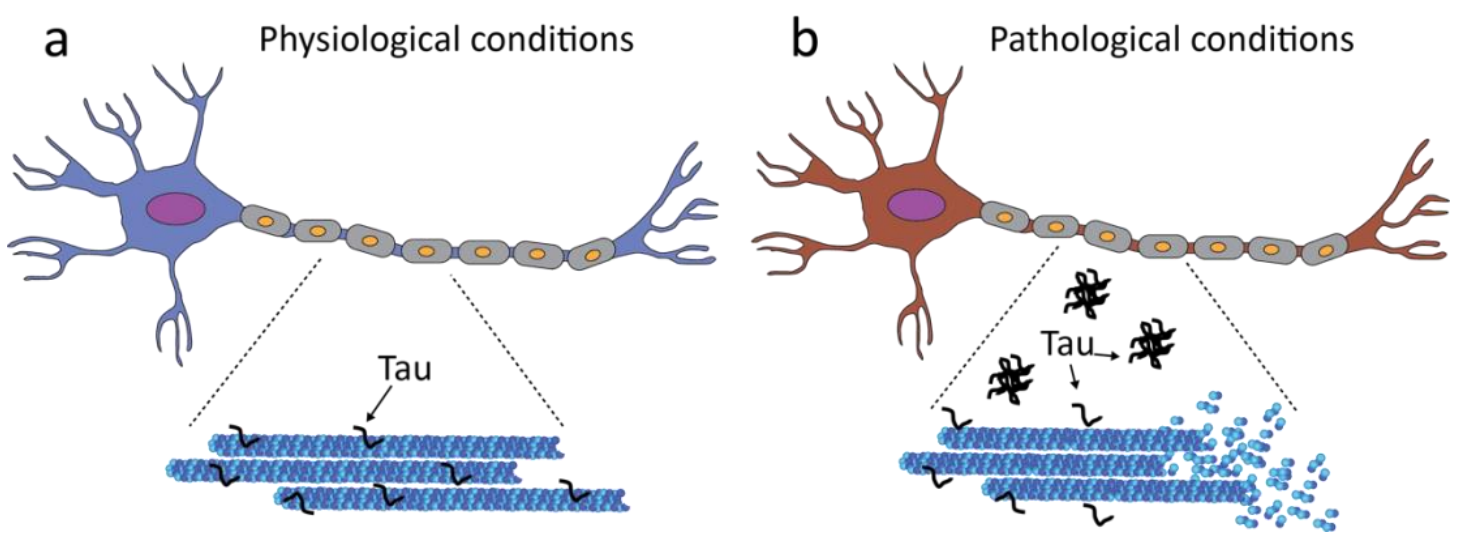

Figure 1.3. Tau in physiological and pathological conditions. a) In healthy neurons, tau interacts with microtubules and assists their dynamic rearrangements in the axons; b) in pathological conditions, neuronal functions are impaired by the misfolding and aggregation of hyperphosphorylated tau proteins, which are detached from the microtubules. Adapted from Sarkar, J Genet, 2018.

Tau aggregation is a common feature in AD, Pick's disease (PiD), Frontotemporal dementia and parkinsonism linked to chromosome 17 (FTDP-17), Progressive Supranuclear Palsy (PSP) and Corticobasal Degeneration (CBD) and other diseases (Table $1)^{26}$. The term "tauopathies" has been coined to classify neurodegenerative disorders in which cognitive impairments are correlated to the accumulation of aggregated tau in diverse areas of the brain ${ }^{15,27}$. Tau fibrils accumulate in intracellular inclusions, e.g. NFTs, Neuropil Treads (NT), Pick's Bodies (PB) or other forms of deposits ${ }^{26}$. Tauopathies can be very heterogeneous in terms of which isoform of tau is aggregated, which neuropathological phenotypes are present and which areas of the brain are affected (Table 1.1). Primary and secondary tauopathies can beclassified according to how many proteins are found aggregated in the inclusions. While in primary tauopathies, tau is the only protein found aggregated in the brain, in secondary tauopathies other proteinaceous deposits are also present ${ }^{28}$. 
Table 1.1 Tauopathies. (Adapted from Lebouvier et al., Curr Opin Neurol (30), 2017)

\begin{tabular}{|c|c|c|c|}
\hline & Name of tauopathy & $\begin{array}{l}\text { Tau } \\
\text { isoform }\end{array}$ & Type of tau inclusions \\
\hline \multirow{7}{*}{ 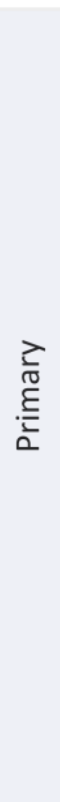 } & Pick's disease & $3 R$ & $\begin{array}{l}\text { Pick bodies, } \\
\text { Pick cells (neurons) }\end{array}$ \\
\hline & Corticobasal degeneration & $4 R$ & $\begin{array}{l}\text { NFTs, } \\
\text { Neuropil treads (neurons), } \\
\text { Coiled bodies (oligodendrocytes), } \\
\text { astrocytic plaques (astrocytes) }\end{array}$ \\
\hline & progressive supranuclear palsy & $4 \mathrm{R}$ & $\begin{array}{l}\text { NFT, } \\
\text { Neuropil treads (neurons), } \\
\text { Coiled bodies (oligodendrocytes), } \\
\text { Tufted astrocytes (astrocytes) }\end{array}$ \\
\hline & Argyrophilic grain disease & $4 R$ & $\begin{array}{l}\text { Grains (neurons), } \\
\text { Coiled bodies (oligodendrocytes), } \\
\text { Thorn-shaped astrocytes (astrocytes) }\end{array}$ \\
\hline & Age-related tau astrogliopathy & $4 R$ & $\begin{array}{l}\text { Thorn-shaped astrocytes, } \\
\text { granular/fuzzy astrocytes (astrocytes) }\end{array}$ \\
\hline & Globular glial tauopathy & $4 R$ & $\begin{array}{l}\text { Globular astrocytic inclusions (astrocytes), } \\
\text { Globular oligodendroglial inclusions (oligodendrocytes) }\end{array}$ \\
\hline & Primary age-related tauopathy & $3 R / 4 R$ & $\begin{array}{l}\text { NFT, } \\
\text { Neuropil treads (neurons) }\end{array}$ \\
\hline \multirow{2}{*}{ 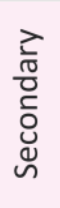 } & Alzheimer's disease & $3 R / 4 R$ & $\begin{array}{l}\text { NFT, } \\
\text { Neuropil treads, } \\
\text { neuritic plaques, A } \beta \text { plaques (neurons) }\end{array}$ \\
\hline & Chronic traumatic encephalopathy & $3 R / 4 R$ & $\begin{array}{l}\text { TDP-43 cytoplasmic inclusions (neurons), } \\
\text { Phospho-tau aggregates (neurons/astrocytes) }\end{array}$ \\
\hline
\end{tabular}

\subsubsection{Tau in Alzheimer's disease}

In 1907, Alois Alzheimer described a case of senile dementia accompanied by specific anatomical changes in the brain observed post-mortem ${ }^{29}$. Today, the majority of people affected by dementia are diagnosed with Alzheimer's disease ${ }^{30}$ and any effort in finding a way to prevent or halt the disease have not succeeded ${ }^{31-33}$.

The disease is described as a multifactorial syndrome where clinical symptoms, e.g memory loss, cognitive decline, depression and in late stages motor dysfunctions ${ }^{27}$ are associated with neuropathological features. Tau pathology in AD is characterized by the formation of NFTs and NTs, described as intracellular inclusions of tau protein fibrils in the neuronal somata and in the dendrites, respectively 34,35 , and it is combined with extracellular deposition of $\beta$-amyloid plaques ${ }^{7}$ (Figure 1.1 and Figure 1.4a). NFTs and NTs are mainly composed of fibrils of misfolded, hyperphosphorylated forms of tau ${ }^{35-38}$, which 
have either a twisted or straight appearance and are thus named Paired Helical Filaments (PHFs) and Straight Filaments (SFs). Once the tau fibrils are formed, they spread in the brain following six stages, previously characterized by Braak and collaborators and known as Braak stages (Figure $1.4 b)^{39}$. These stages are also correlated to the disease progression, indicating that more than 20 years can separate the onset of the neuropathology of the disease from its phenotypical manifestation.

a

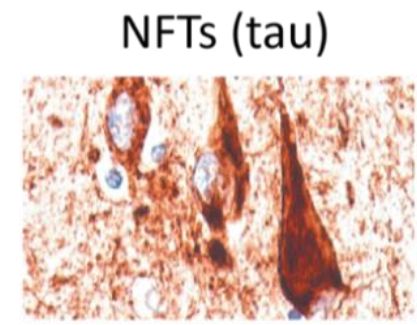

b Transenthorinal

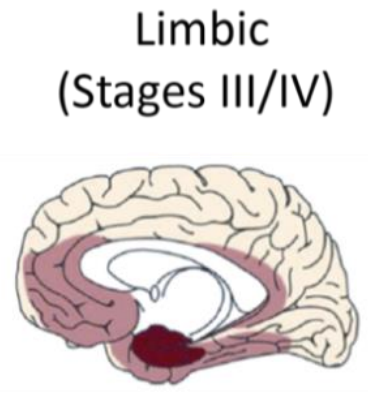

SPs $(A \beta)$

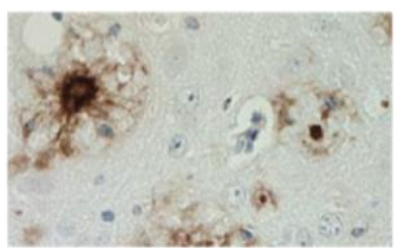

b
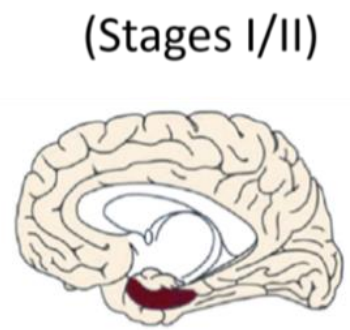

Neocortical

(Stages $\mathrm{V} / \mathrm{VI}$ )

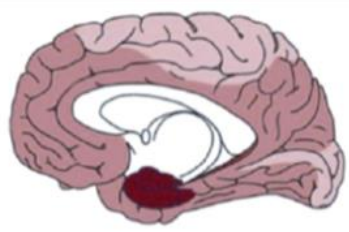

Figure 1.4. Tau pathology in AD. a) Neuropathological hallmarks of AD: tau aggregation in NFTs is found in the brain of $A D$ patients together with amyloid plaques composed of mainly the $A \beta$ protein. NFTs taken from Kovacs, Handb Clin Neurol, 2017; SPs taken from Savastano et al., J Alzheimers Dis, 2015. b) Schematic reproduction of the Braak stages, which show the spread of tau pathology through the brain. Adapted from Scholl et al., Mol Cell Neurosci, 2019.

\subsection{The microtubule-associated protein tau}

\subsubsection{Tau isoforms and domain organization}

First characterized as a microtubule-associated protein ${ }^{40,41}$, tau became an interesting target once its presence in PHFs/SFs was confirmed ${ }^{35-38}$. In particular, tau started to be considered as a disease-causing agent after the identification of tau mutations in frontotemporal dementia ${ }^{42}$. 
The tau sequence is encoded by the MAPT gene on the chromosome $17^{43}$. Six isoforms are expressed in the neurons as a result of alternative splicing of exons 2, 3 and $10^{44}$. Each isoform differs in sequence composition and their nomenclature is based on the domains that are present in the primary sequence ${ }^{44}$ (Figure 1.5).

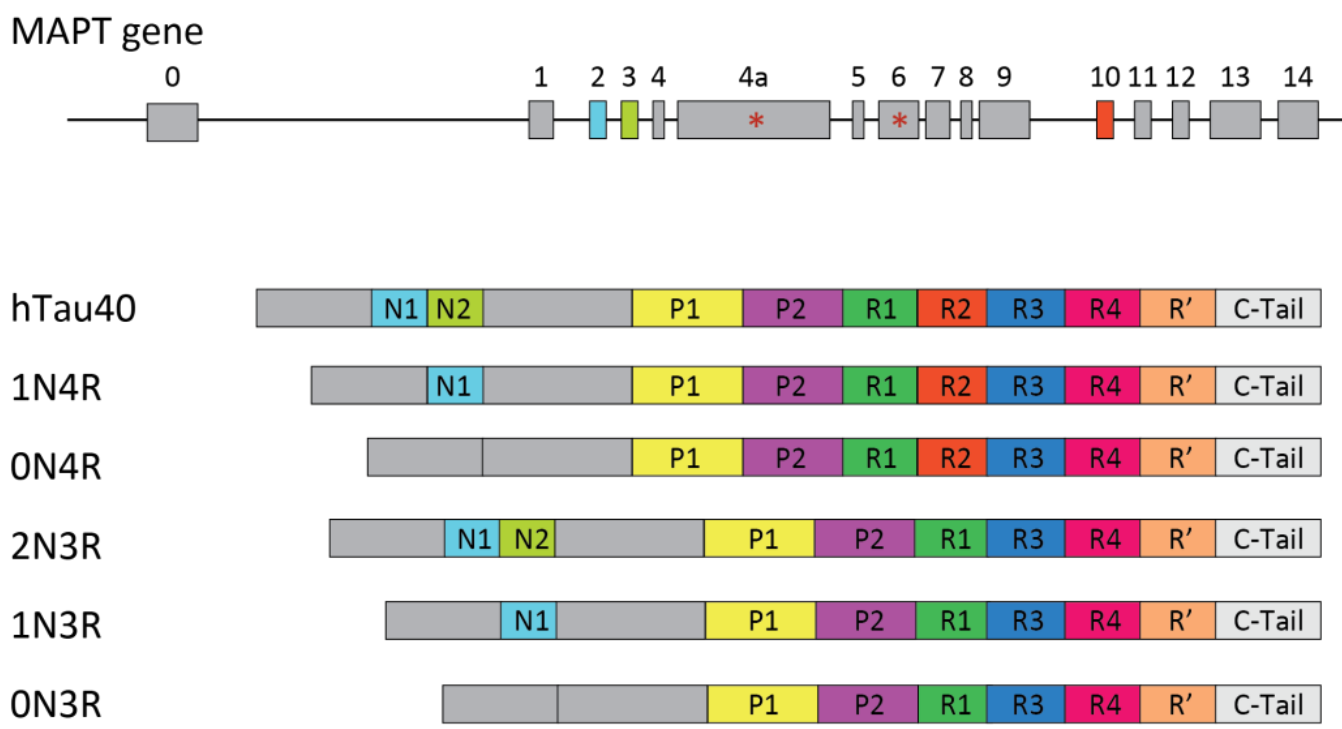

Figure 1.5. Tau isoforms expressed in the brain. Exons 2,3 and 10 are included or not in the tau sequence via alternative splicing. The exons $4 a$ and 6 , indicated with asterisks, are only included in the sequence of tau isoforms expressed in the peripheral nervous system. Adapted from Martin et al., Neurochem Int 2011.

The isoform htau40, is a protein of $45.9 \mathrm{kDa}$ comprising two insertions of 29 amino acids each ( $\mathrm{N} 1$ and N2), a proline-rich region, five pseudo-repeats (from R1 to R4 and R') and a C-terminal tail. The shortest isoform of tau, the embryonal htau23, comprises only three repeats and lacks the $\mathrm{N}$-terminal domain. Isoforms containing all pseudo-repeats are conventionally indicated as $4 R$, while those lacking the $R 2$ domain are defined as $3 R^{16}$. Tau is equally distributed in different areas of the brain ${ }^{37}$ and the expression of the six isoforms is regulated according to the development of the nervous system ${ }^{20,45}$ : the expression of htau23 is up-regulated in the fetal human brain ${ }^{44}$, while at mature stages of development it is down-regulated in favour of the $4 \mathrm{R}$ isoform ${ }^{26}$.

The different regions of tau sequence are shown in figure 1.6, together with a description of their activities. The $\mathrm{N}$-terminal region interacts with the neuronal membrane and acts as a phosphatase-activating domain (PAD) ${ }^{46}$. With respect to the 
interaction with microtubules, the domain is defined as a projection domain (PD), because it is not directly engaged in the interaction.

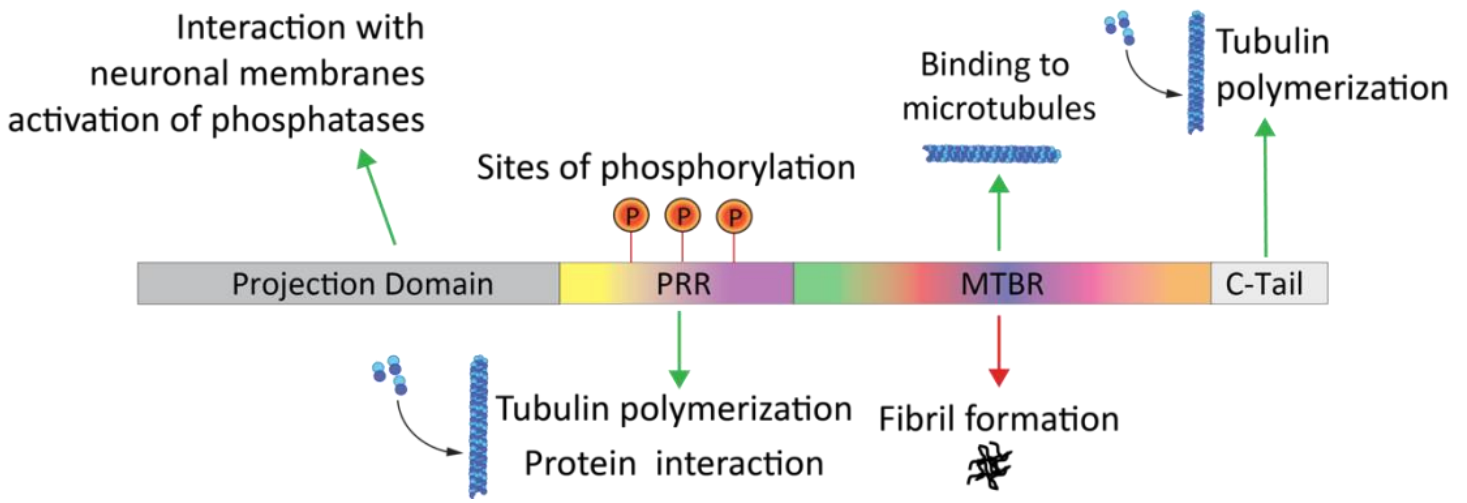

Figure 1.6. Tau sequence regions and their role in tau activity. Schematic representation of the tau sequence including the $\mathrm{N}$-terminal projection domain, the proline-rich region (PRR), the microtubulebinding region (MTBR) and the C-terminal tail. For each domain, some of its activities are schematically shown. The projection domain interacts with neuronal membranes and activates phosphatases. The PRR is involved in interactions with other proteins and in microtubule polymerization. This region is rich in serine and threonine residues, which are targeted by proline-directed kinases: the phosphorylation of this region is important for the regulation of protein-protein interactions and tau functions, it can also have a negative effect on tau physiological activity. The MTBR interacts with microtubule and actin filaments and under pathological conditions it is involved in the fibril formation. The C-terminal tail contributes to the polymerization of tubulin.

Following this domain, the proline-rich region (PRR), which can be subdivided into the $\mathrm{P} 1$ and the $\mathrm{P} 2$ regions, plays an important role for the interaction of tau with tubulin and is involved in signaling pathways: the prolin-rich motifs (PRM, i.e. PxxPxxP motif) within the sequence are recognized by proteins containing prolin-rich motifs binding modules (PRM-binding modules) like the $\mathrm{SH} 3$ domain $^{47}$. In addition, the presence of numerous serine and threonine residues makes the proline-rich region a target of several kinases, some of which are also related to the pathological aggregation of tau ${ }^{48,49}$. The Cterminus comprises four pseudo-repeats(from R1 to R4) plus a weakly homologous repeat region termed $R^{\prime}$. Together these domains constitute the microtubule binding region (MTBR), responsible for the interaction with tubulin and microtubules, actin and 14-3-3 proteins and involved in the pathological formation of tau fibrils. The C-terminal tail 
contributes, together with the proline-rich region, to the polymerization of microtubules ${ }^{16,50,46}$. Several artificial tau constructs have been produced in the past years, in order to better study its physiological and pathological aspects ${ }^{50}$ : K18 (residues Q244K369) comprises the four pseudo-repeats (from R1 to R4) while K19 lacks the R2 repeat ${ }^{51}$; K32 comprises the pseudo-repeats, the flanking P2 domain and R' (residues S198-S400) ${ }^{52}$. Figure 1.7 shows the domain organization of tau and the mentioned constructs, which have also been used in this work.

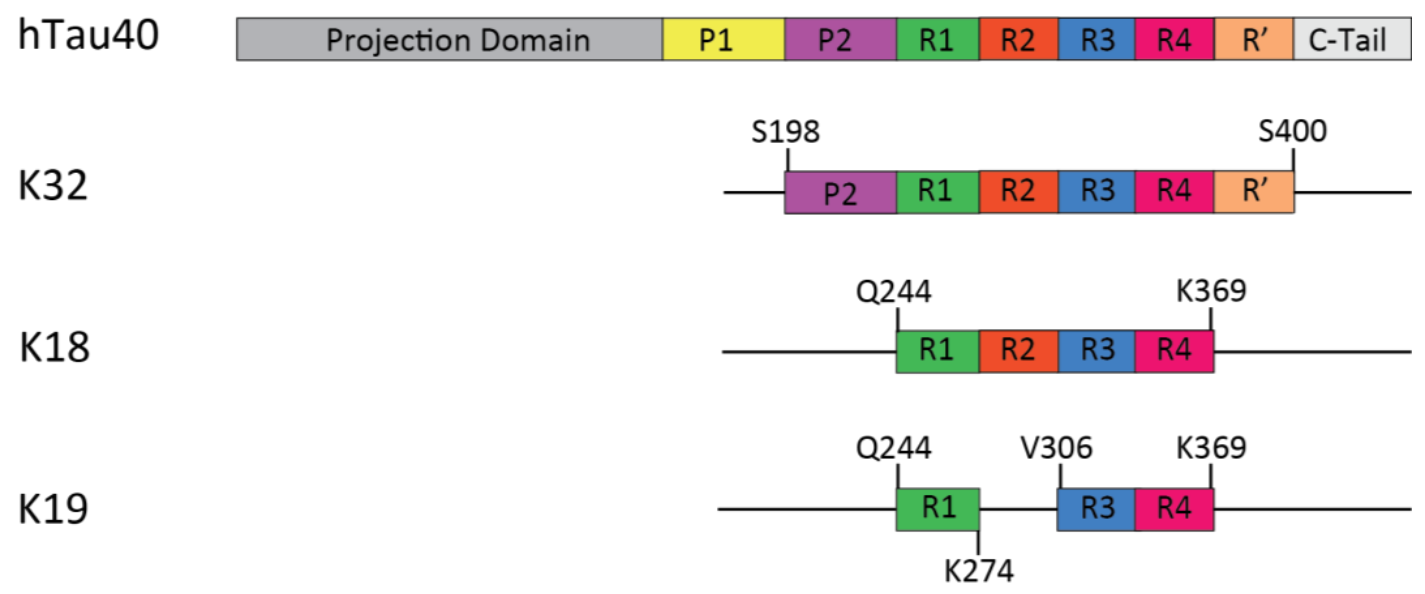

Figure 1.7. Tau constructs. The domain organization of three tau constructs is represented together with that of the full-length protein. K32 comprises the P2 domain of the proline-rich region and the MTBR (domains from R1 to R'); K18 comprises all the four pseudo-repeats regions while K19 comprises only the domains R1, R3 and R4.

\subsubsection{The intrinsically disordered properties of Tau}

Intrinsically disordered proteins (IDPs) are characterized by the lack of a defined secondary structure and preferentially adopt random coil conformationsin solution ${ }^{53}$. IDPs are heat stable and resistant to acidic treatments, their sequence is characterized by a small number of hydrophobic residues and by low complexity of the amino acid composition ${ }^{54}$. Tau displays intrinsically disordered properties ${ }^{55,56}$. In NMR studies, tau spectra are characterized by little chemical shift dispersion ${ }^{56}$, typical of unfolded proteins and IDPs ${ }^{57}$. The protein is rapidly exchanging between different conformations, with some distinct regions temporarily adopting $\alpha$-helical or $\beta$-sheet-like conformations ${ }^{56}$. According to its aminoacidic composition, the protein is hydrophilic with a basic character and an 
isoelectric pointof around 9-10 16 . Serine, threonine and positively charged residues, e.g. lysine, are the most represented in the sequence. Overall, the protein is positively charged while the $\mathrm{N}$-terminal domain carries a negative net charge at neutral pH (Figure 1.8) ${ }^{16,56}$.

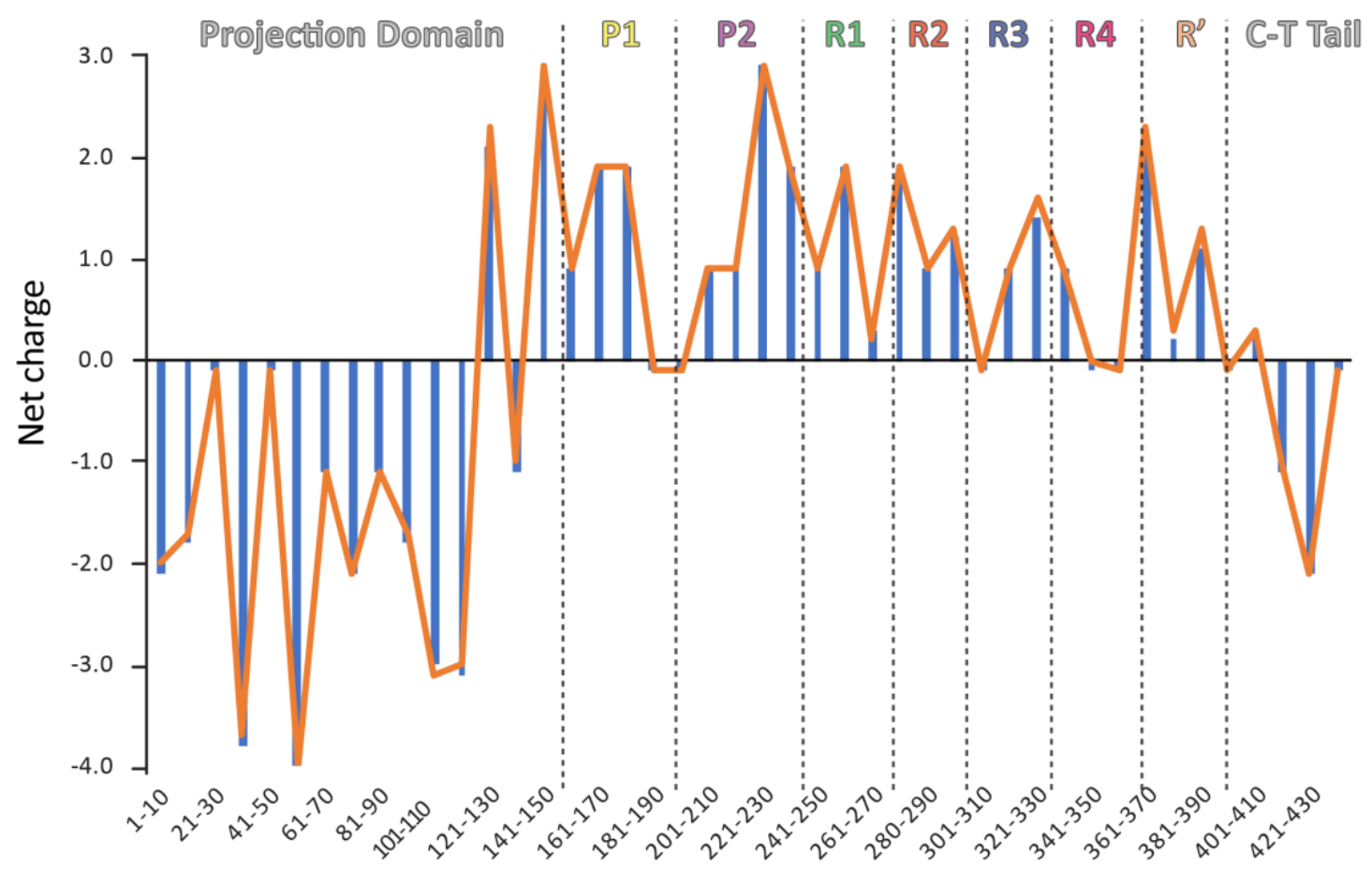

Tau sub-peptides (10 aa)

Figure 1.8. Charge distribution in the tau sequence. The values were obtained by dividing the sequence into sub-peptides of 10 amino acids and calculating their net charges at pH 7.0 (blue histograms) and pH 6.8 (orange line). The figure shows that the $\mathrm{N}$-terminus is negatively charged while the rest of the sequence is mainly positively charged. The organization of tau domains is also included, in order to correlate the net charge to each domain. The PPR and the MTBR are positively charged, while the last portion of the C-terminal tail has a negative net charge.

\subsection{Biomolecular liquid-liquid phase separation}

Liquid-liquid phase separation (LLPS) is a phenomenon which describes the de-mixing of the components in a complex fluid into two phases ${ }^{58}$. For simplicity, if a system composed by a solute, e.g. a polymer, and a solvent is taken into consideration, the free energy associated to its mixing will be defined by the entropy of the mixing, which will favor the formation of a homogenous phase, and by the interactions established in the system, e.g. 
solute-solute and solute-solvent interactions ${ }^{58,59}$. From the entropic point of view, the mixing to a single phase is energetically favored while the de-mixing represents a penalty ${ }^{60}$. If the interactions, e.g. electrostatic, hydrophobic interactions, established between the molecules of the solute, or between solute-solvent molecules, counterbalance the entropic penalty deriving from the de-mixing, then the separation into two phases occurs. As an effect of this, one phase will contain high concentration of the phase-separated solute while the other phase will contain the solvent.

\subsubsection{LLPS in the cellular environment}

LLPS has started to be considered a phenomenon at the base of the regulation of many biological processes ${ }^{58}$.The cellular environment is rich in membraneless compartments, containing different proteins and nucleic acids, which regulate many cellular processes, e.g. DNA damage repair, splicing and cellular signaling ${ }^{61}$. Phase-separation drives the formation of these compartments, which are also named biomolecular condensates ${ }^{62}$, e.g. nucleoli, P-granules, stress granules and Cajal bodies ${ }^{63,64}$. In vivo, LLPS is driven by multivalent interactions between different cytoplasm components, e.g. DNA, RNA and proteins. The proteins which undergo phase separation exhibit low complexity domains within the sequence and/or intrinsically disordered properties. Intrinsically disordered regions promote phase-separation via homotypic interactions, while negatively-charged molecules, e.g. RNA, contribute to the process through electrostatic interactions ${ }^{59}$. The amino acid composition of phase-separating proteins also contributes to different extents, e.g. the side chains of aromatic residues interact with basic ones via cation- $\pi$ interactions, charged residues contribute to the establishment of electrostatic interactions ${ }^{65,66}$. Biomolecular condensates have a liquid-like behaviour, which is described as (i) acquisition of a spherical shape, (ii) fusion after interaction of two condensates and (iii) internal rearrangement, observed by photobleaching and recover of the signal ${ }^{58}$.

Many proteins, some of them related to neurodegeneration, phase-separate in the cytoplasm, e.g. the ALS-related protein $\mathrm{FUS}^{67}, \mathrm{RNA}$-binding proteins ${ }^{68}$ and the protein Ddx4 present in the stress granules ${ }^{69}$. 


\subsubsection{LLPS in vitro}

In order to study this phenomenon, phase-separation of proteins has been reproduced in vitro and analysed using optical microscopy techniques, e.g. differential interference contrast (DIC) and fluorescence microscopy, and biophysical approaches, e.g. the fluorescence recovery after photobleaching (FRAP) technique ${ }^{66,68,70}$. In order to promote phase separation of a certain protein and to mimic the crowding of the cellular environment, crowding agents like polyethylene glycol (PEG) or dextran are employed ${ }^{71}$. Also charged molecules like RNA are often employed in LLPS studies, due their presence in many biomolecular condensates in vivo as well as the electrostatic interactions they can establish with other proteins ${ }^{59}$. Temperature, critical concentration and other conditions at which phase separation occur change according to the protein characteristics.

\subsubsection{Tau LLPS}

Tau displays intrinsically disordered properties and shows low complexity in its amino acid composition. Positively charged residues such as lysines drive tau LLPS and regulate the formation of liquid-like droplets, suggesting that intra-molecular electrostatic interactions are an important factor in tau LLPS ${ }^{65,66}$. On top of that, tau post-translational modifications, e.g. serine and tyrosine phosphorylation and acetylation of lysine residues, play a role in modulating tau phase-separation, most likely by changing the charge distribution $65,70,71$.

In vitro studies on tau phase separation reported that the K18 construct, comprising the MTBR, self-coacervates at high protein concentration and at $37-45^{\circ} \mathrm{C}$ without additional cofactors. The addition of heparin to the self-coacervated K18 droplets resulted in fibril-like structures, suggesting that the combination of high concentration and aberrant phase separation might initiate fibrillization of tau ${ }^{70}$. Thus, in pathological conditions the combination of tau hyperphosphorylation, high concentrations of tau in droplets and the recruitment of polyanions, e.g. heparin, might favor its aggregation into fibrils ${ }^{70}$ (Figure 1.9, left side of the panel). LLPS of htau40 has also been observed at room temperature and in presence of either crowding agents or negatively charged molecules, 
e.g. poly-U RNA ${ }^{66,70,71}$. Recently, tau LLPS has been correlated to its physiological function of interaction with tubulin and microtubules ${ }^{71,72}$ (Figure 1.9, right side of the panel). Fluorescence microscopy has shown that tau interacts withthe microtubules surface forming condensates: these condensates share some of the liquid-like properties observed in the droplets and are considered to be involved in the interaction with motor proteins located on the microtubules surface ${ }^{72,73}$.On top of that, Hernandez-Véga recently reported that droplet formation is related to its ability to regulate tubulin polymerization $^{71}$, while Wegmann and collaborators reported the phase separation of htau40 expressed in primary cortical mouse neurons ${ }^{74}$. The authors proposed a model in which tau phase separation of phosphorylated and/or mutated forms might represent an initial step of its aggregation.

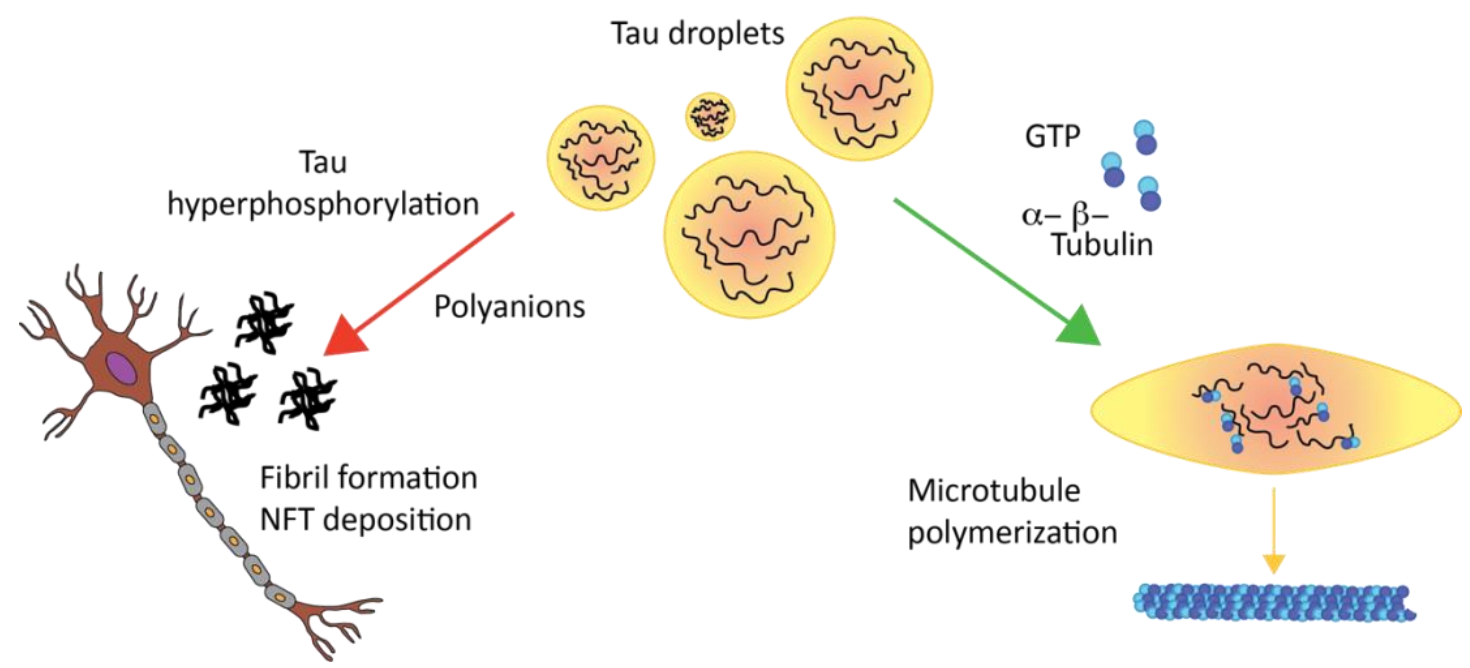

Figure 1.9. Tau LLPS in physiological and pathological conditions. Upon formation of tau droplets, microtubule filaments polymerize due to the interaction between tau and $\alpha \beta$-tubulin; the recruitment of heparin into a high-tau-concentrated environment and tau hyperphosphorylation might favour the formation of fibrils, leading to the deposition of NFTs and other sort of inclusions in the neurons.

\subsection{Tau fibrils}

One of the first neuropathological features described by Alois Alzheimer was the presence of aggregates in brain tissue ${ }^{29}$. This fibrillated material was later discovered to be the NFTs, agglomerates of PHFs of tau ${ }^{35,36}$. A wide range of studies has since then been performed, in order to characterize tau fibrils with the hope that understanding their molecular and structural basis could lead to a cure for AD and other tauopathies. 


\subsubsection{Amyloid fibrils and their polymorphism}

Amyloid fibrils are insoluble filamentous aggregates of misfolded proteins, biophysically characterized by an X-ray cross- $\beta$ fiber pattern of diffraction, i.e. an inter-strand distance of $4.8 \AA$ and a space between two $\beta$-sheets of $10 \AA^{75}$ (Figure 1.10a). Fibrils are composed of two or more protofilaments held together by weak interactions; each protofilament consists of two $\beta$-sheets extending perpendicularly along the fibril growth axes ${ }^{75}$. The strands within the $\beta$-sheet are tightly bound by hydrogen bonds between the amide and carboxyl groups in the backbone with a parallel orientation ${ }^{4}$ (Figure $1.10 \mathrm{~b}$ ).

a

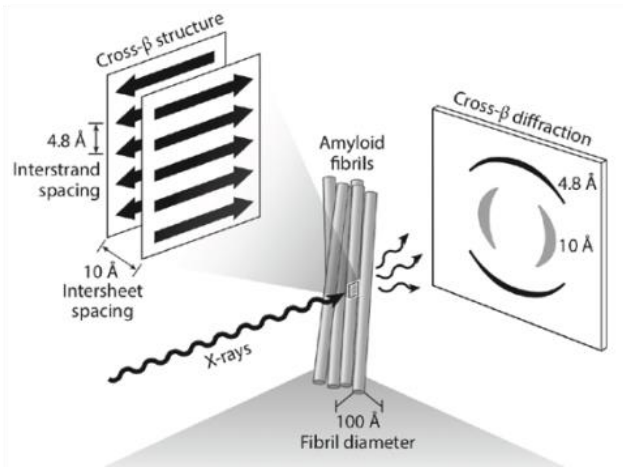

b

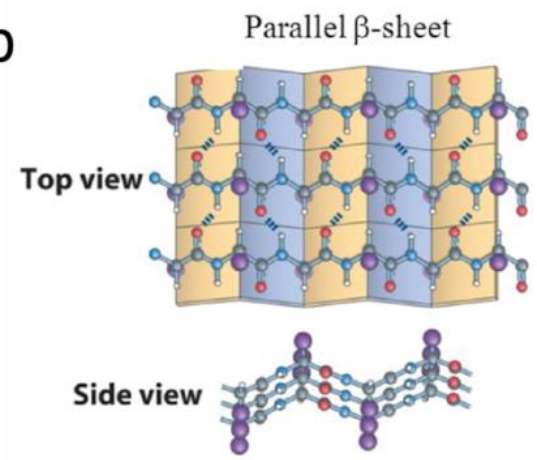

Figure 1.10. Cross- $\beta$ structure of amyloid fibrils. a) The $X$-ray diffraction pattern shows an inter-strand spacing of $4.8 \AA$ and an inter-sheet spacing of $10 \AA$. Adapted from Einsenberg and Sawaya, Annu Rev Biochem, 2017. b) Parallel orientation of $\beta$-sheets viewed from the top and from the side. Taken from Lehninger Principles of Biochemistry.

The way in which protofilaments arrange to give amyloid fibrils, the type of bonding established between the strands, the conditions under which the growth occurs and other factors can generate different cross- $\beta$ structure. This phenomenon has been described as fibril polymorphism ${ }^{76,77}$, by which the same misfolded protein can generate fibrils of different and heterogeneous structure. The fibril polymorphism is starting to be correlated with different pathologies and neurodegenerative disease types ${ }^{78}$, for example in $A D$ and PiD two different three-dimensional structures of tau fibrils could be defined via cryo-EM ${ }^{79,80}$. Although a physiological role for many amyloid fibrils has been reported $^{76}$, the focus has been centered on the study of those amyloidogenic proteins involved in neurodegenerative diseases. 


\subsubsection{Tau aggregation}

One of the unexplained aspects regarding tau is why it aggregates at all: tau is a very stable protein, with heat and acid treatment resistance ${ }^{55}$ and it has a very low aggregation propensity in vitro.

In vitro aggregation of tau has been traditionally achieved using polyanions like heparin ${ }^{51,81}$ and arachidonic $\operatorname{acid}^{51}$. Negative charges play an essential role in the aggregation process by neutralizing the positive charges on tau (Figure 1.8), thus favoring tau self-assembly. Several studies have been directed towards the identification of the protein domains responsible for its aggregation ${ }^{82-86}$.

Tau fibrils are characterized by a solvent inaccessible rigid core and a fuzzy coat, which can be removed by protein digestion ${ }^{35}$. Biochemical studies showed that the rigid core of the fibrils is composed by the pseudo-repeat region and that the hexapeptide ${ }^{306}$ VQIVYK $^{311}$ at the beginning of the R3 domain represents the minimal sequence able to self-assemble into fibrils ${ }^{85}$. Studies with small tau constructs evidenced a similar role for the hexapeptide ${ }^{275}$ VQIINK ${ }^{280}$ in the R2 domain to form bona fide fibrils ${ }^{51,87}$ (Figure 1.11). NMR analysis of tau secondary structure in solution reported residual $\beta$-sheet structure for the two hexapeptides ${ }^{82}$ : these were hypothesized to provide hydrophobic contributions that in pathological conditions can enhance protein aggregation. The fuzzy coat, composed by the $\mathrm{N}$-terminal domain, the proline-rich region and the $\mathrm{C}$-terminal domain, maintains a higher degree of flexibility in the fibrils. Nevertheless transient contacts between the proline-rich region and the core of the fibrils could be detected using NMR spectroscopy ${ }^{83}$ supporting its possible roles in tau aggregation ${ }^{88}$.

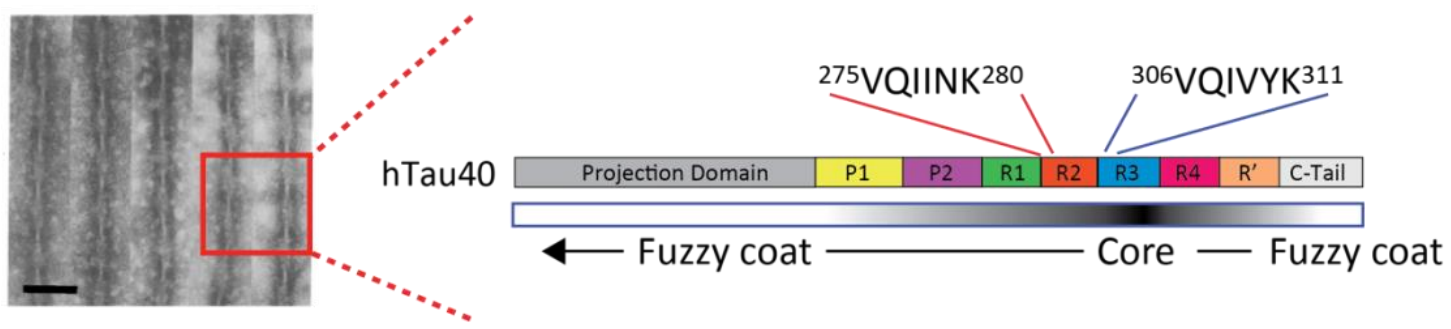

Figure 1.11. PHF of tau. The electron micrograph shows negatively stained fibrils of tau with twisted morphology (scale bar $100 \mathrm{~nm}$, adapted from Wischik et al., Proc natl Acas Sci U S A, 1988). The fibril edges are blurry due to the presence of the fuzzy coat, which can be removed by pronase treatment. A schematic representation of tau domains is shown, with the sequence of the two hexapeptides in R2 and R3: these amino acid stretches have been considered to be important for the aggregation process ${ }^{56,85}$. 
The factors that induce tau aggregation are not clearly understood yet. Post-translational modifications alter tau affinity to microtubules by altering its charges. In early studies concerning the composition of NFTs, hyperphosphorylated tau was extracted from PHFs therefore a major contribution to enhanced aggregation propensities was attributed to aberrant phosphorylation on serine and threonine residues ${ }^{23,89}$, e.g. phosphorylation is considered to favour aggregation ${ }^{90}$ by detachment of tau from microtubules and increasing the concentration of unbound tau in the cytosol. Among the post-translational modifications increasing tau tendency to aggregate, single-point mutations leading to the loss of K280 facilitates self-assembly and provokes toxicity ${ }^{91,92}$. In addition, oxidation of C291 in R2 and C322 in R3, occurring both in vivo and in vitro, allows for the formation of intermolecular disulfide bridges between tau molecules, thus enhancing self-assembly 93,94. It became clear that the characterization of the three-dimensional structure of tau fibrils would have had an essential impact for a better understanding of the mechanisms driving tau aggregation in the brain.

\subsubsection{The structure of tau fibrils}

Solid-state NMR (ssNMR) spectroscopy, electron microscopy, X-ray diffraction, electron paramagnetic resonance (EPR) and other biophysical techniques have been employed to gain insight into the structure of tau fibrils ${ }^{75,79,80,82-84,86,95}$. One of the challenging aspects that has made the structural characterization difficult is the observation that tau fibrils are heterogeneous ${ }^{77,96}$.

Recently three-dimensional structures for fibrils of tau purified from patients pathologically confirmed with $A D$ and PiD were determined using cryogenic EM (cryo$E M)^{79,80}$ (Figure 1.12): different ultra-structures were observed for the two diseases, leading to the consideration that a pathological phenotype might be linked to the molecular structure of the fibrils ${ }^{78}$. Tau fibrils purified from the brain of an AD patient exhibited two morphologies, PHFs displayed a periodical twist of $80 \mathrm{~nm}{ }^{16}$ while SFs did not. Both types of fibril are composed by two C-shaped protofilaments composed of the R3 and R4 domains, whose amino acids form eight $\beta$-sheets in total. The hexapeptide ${ }^{306}$ VQIVYK $^{311}$ is engaged in the formation of a cross- $\beta$ sheet structure with residues in the $R^{\prime}$ region. The inter-repeat PGGG motif at the end of the $R 3$ domain forms a turn, which gives the C-shape to the structure. The PGGG motif at the end of R4 creates the interface 
by which the two protofilaments interact with each other: the interaction between the identical PGGG motifs is symmetrical in the PHFs and it is asymmetrical in SFs (Figure 12a), thus the different arrangement of two protofilaments is the source of the two distinct polymorphs.

a

AD PHF

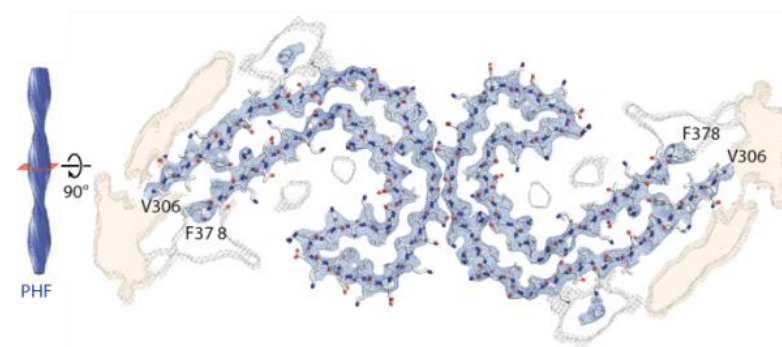

b

PiD NPF

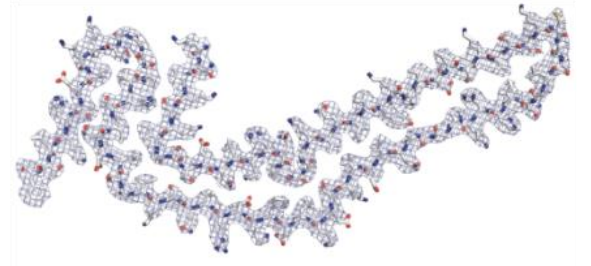

AD SF

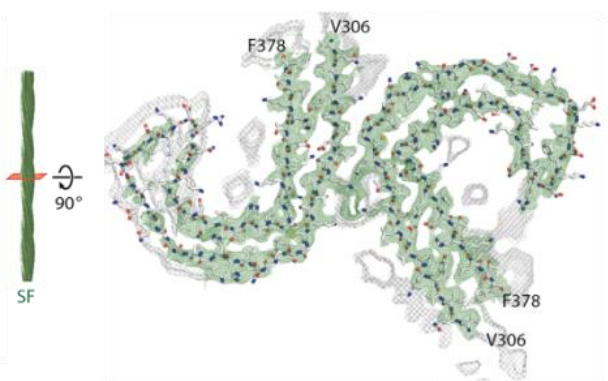

C 1-MAE......SRL-243
Pick 244-QTAPVPMPDLK-NVKSKIGSTENLKHQPGGGK-274 (R 1$)$ 275-VoIINKKLDLS-NVOSKCGSKDNIKHVPGGGS-305 (R2) 306-VQIVYKPVDLS-KVTSKCGSLGNIHHKPGGGQ-336 (R3) Pick 337-VEVKSEKLDFKDRVQSKIGSLDNITHVPGGGN-368 (R4) Pick
Alz. 369- KKIETHKLTFRENAKAK.....QGL-441 Pick

d
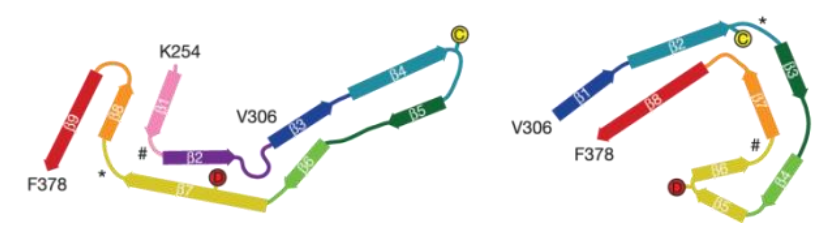

Figure 1.12. Cryo-EM structures of tau fibrils purified from AD and PiD brain. a) Alzheimer fold of PHFs and SFs. Two C-shaped protofilaments, composed of the R3 and the R4 domains, constitute the fibrils: the different morphology originates from the way the protofilaments interface with each other. Information regarding the rest of the tau sequence could not be obtained, thus suggesting that the most rigid core of the fibrils is composed of the R3 and the R4 domains ${ }^{79}$. b) Pick's disease fold of the NPFs. The fibrils are composed of a single protofilament made of $\beta$-sheets of residues from the R1, R3 and R4 ${ }^{80}$. The $\beta$-sheets have a different arrangement with respect to the AD fold (d) although the sequence involved in the fibrils formation is the same (c), thus suggesting that the heterogenous morphologies originate from different rearrangements of the $\beta$-sheets (taken from Fitzpatrick et al., Nature $2017^{79}$ and Falcon et al., Nature 2018).

The resolution quality up-stream to the R3 repeat and downstream to the R4 repeatprevented insights into other parts of the protein. Indeed, additional electron 
density at the $\mathrm{N}$ - and $\mathrm{C}$-termini of the cross- $\beta$ structure suggested that additional amino acids from the R1/R2 and $R^{\prime}$ pseudo-repeats, respectively, could contribute to the structure of tau fibrils. On the other hand, the R2 domain was not considered part of the core because of its cleavage by pronase. Although there is evidence supporting contacts with the core of the fibrils ${ }^{83}$, the cryo-EM analysis also did not provide information about the structural properties of the proline-rich region ${ }^{79}$.

Fibrils purified from the brain of a PiD patient were composed of the tau $3 R$ isoform, which lacks the R2 domain. Two morphologies defined as Narrow Pick filaments (NPFs) and Wide Pick filaments (WPFs) could be distinguished. The NPFs are composed of a single protofilament, whose core is made of nine $\beta$-sheets provided by the R1, R3 and R4 repeats. The filament adopts a hairpin-like shape via hydrophobic interactions between the strands ${ }^{80}$ (Figure 1.12b). The WPFs are composed of two NPFs associated together. In PiD, tau isoforms containing three pseudo-repeats are the most expressed (Table 1.1), therefore the R2 domain was not modeled in the cryo-EM structure.

Again with the use of cryo-EM, three-dimensional structures of heparin-induced in vitro tau fibrils have also been characterized ${ }^{97}$. The resulting structures differed from the $A D$ and PiD structures and included the $R 2$ domain in the core of the fibrils ${ }^{79,80}$. In any of the structures resolved by cryo-EM, the proline-rich region has never been observed and no information could be provided, leaving open questions regarding the contribution of this domain to the formation and structure of tau fibrils.

\subsection{Tau interaction with the cytoskeleton}

In section 1.1.1, tau has been introduced as a member of the MAP family, consistent with its function to bind microtubules and regulate their polymerization ${ }^{40,41}$. In $A D$, microtubule disruption results in axonal transport impairments and can induce neuronal cell death ${ }^{3}$. For this reason, the nature of tau interaction with microtubules, and recently with soluble tubulin, has been investigated using different approaches, including biochemical studies, nuclear magnetic resonance (NMR) spectroscopy, cryo-EM and others ${ }^{19,50,52,56,98-101}$. The following sections summarize some of the findings regarding the involvement of tau in polymerizing tubulin and binding to microtubules. 


\subsubsection{Tubulin and microtubules}

The family of eukaryotic tubulin is composed by $\alpha-, \beta-$, and $\gamma^{-t u b u l i n}$ ( $55 \mathrm{KDa}$ each). The $\gamma$-tubulin is organized in rings, which form the microtubule-organizing centers (MTOC). The $\alpha$ - and $\beta$-tubulin isoforms create a heterodimer which constitutes the basic unit of the microtubule protofilaments ${ }^{102}$. Tubulin monomers have a GTP-binding site and exhibit GTP catalytic activity, which is necessary for polymerization ${ }^{102}$. Heterodimers of $\alpha$ - and $\beta$ tubulin polymerize into protofilaments, which further assemble into hollow and cylindrical microtubules ${ }^{102,103}$ (Figure 1.13). Microtubule filaments are in a constant dynamic polymerization and depolymerization equilibrium, termed "Dynamic instability" 104. This allows them to assist the cell in processes, which require fast rearrangements in the cytoskeleton ${ }^{102}$. In neurons, microtubules are responsible for trafficking of neurotransmitters, synaptic vesicles and catabolites via anterograde transport. Their interaction with tau in the axons is considered important for a correct functioning.

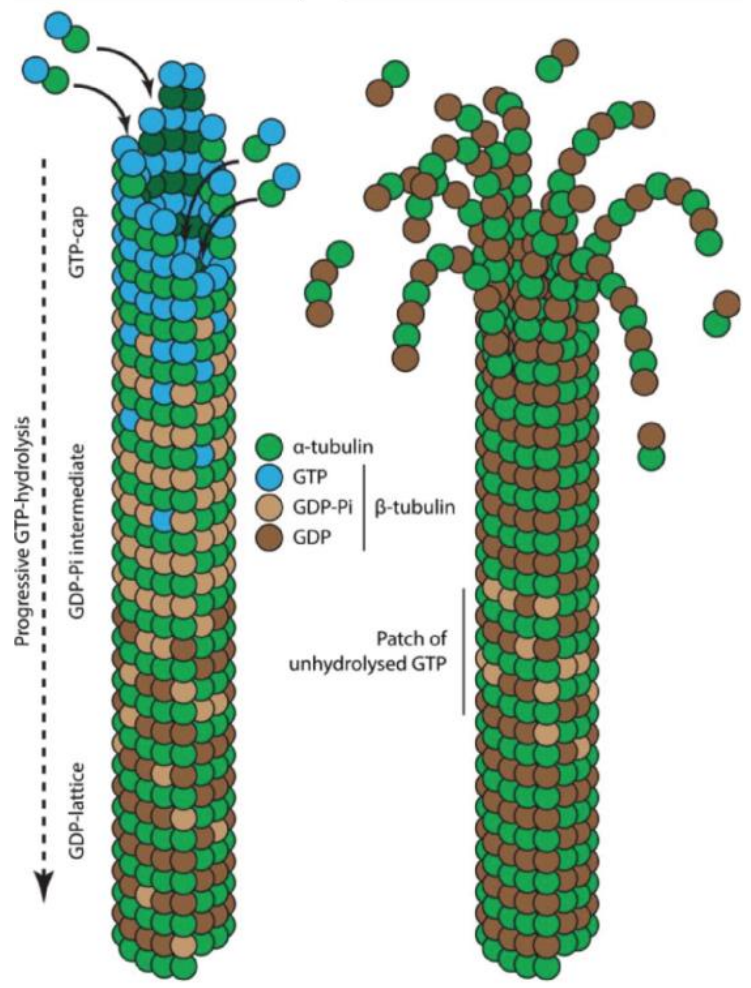

Figure 1.13. Schematic representation of the microtubule filament organization. $\alpha \beta$-tubulin heterodimers polymerize into protofilaments upon GTP hydrolysis. Microtubules are formed by the assembly of 13 protofilaments and are in a dynamic equilibrium of polymerization and disassembly. Because of the head-to-tail polymerization of $\alpha$ - and $\beta$-tubulin, microtubules show a polarity, with a minus-end exposing the $\alpha$-tubulin and a plus-end exposing the $\beta$-tubulin. Of the two extremities, the plusend is the one showing dynamic behavior ${ }^{103}$. Taken from Vleugel et al., Cell Adh Migr, 2016. 


\subsubsection{Tau regions involved in the interaction with tubulin and microtubules}

The pseudo-repeats, the proline-rich region and the C-terminal tail of tau are important for the interaction of tau with tubulin and microtubules ${ }^{50,99,105-107}$. The ability of the pseudo-repeats to bind to microtubules has been observed in biochemical studies $^{19,50,90,105}$ and by NMR spectroscopy ${ }^{56,98,108}$. Using a combination of biochemical assays and light scattering experiments, Gutske et al. reported that in full-length tau the repeat region exhibits a high affinity for microtubules ${ }^{50}$. At the same time, in constructs comprising only 4 or 3 pseudo-repeats, the binding was relatively weak ${ }^{50}$. In the same work, the Mandelkow group also reported that the presence of the proline-rich region in different constructs enhanced tau affinity to microtubules ${ }^{19}$. This effect was extended to the C-terminal tail as well. Following this, a model was proposed, in which the flanking regions act as "claws" facilitating tau docking on the microtubules surface while the pseudo-repeats act as a catalytic domain, regulating microtubule polymerization ${ }^{19,52}$. In ${ }^{1} \mathrm{H}-{ }^{15} \mathrm{~N}$ HSQC NMR experiments, the repeat region shows a loss of intensity in presence of microtubules, indicating their involvement in the interaction ${ }^{56}$. Because of their amino acid composition, the interaction between the repeats and microtubules include hydrophobic interactions ${ }^{56}$. The two hexapeptides, ${ }^{275}$ VQIINK $^{280}$ and ${ }^{306}$ VQIVYK $^{311}$, present in the repeats R2 and R3, respectively, are also involved in tau fibril formation (see section 1.4.2), suggesting a link between physiological and pathological roles of tau.

The proline-rich region is not only involved in the interaction with microtubules but is also considered to play a major role in tubulin polymerization ${ }^{105}$. The ${ }^{225} \mathrm{KKVAVVRT}^{231}$ sequence, in the $\mathrm{P} 2$ domain of the proline-rich region, provides important hydrophobic contributions ${ }^{52,56}$.

\subsubsection{Tau-induced tubulin polymerization}

The first evidence that tau can polymerize tubulin was reported by Weingarten et al. ${ }^{109}$. Biochemical studies support a central role of the proline-rich region in tubulin polymerization ${ }^{46,48,49,52,105}$. Goode and co-workers reported $\mathrm{N}$-terminal truncation studies performed on tau, which indicated that the microtubule-binding activity of the prolinerich region is located between the residues $\mathrm{K} 215$ and the $\mathrm{N} 246$, with the ${ }^{225} \mathrm{KVAVVRT}^{231}$ 
sequence strengthening the interaction ${ }^{105}$. Despite the weak binding affinities to microtubules reported for the proline-rich region, when this was combined with at least one domain from the pseudo-repeat region microtubule assembly was enhanced ${ }^{105}$. Consistent with these observations, Mukrasch et al. reported NMR studies indicating that the ${ }^{225} \mathrm{KVAVVRT}^{231}$ sequence is involved in the binding of tau to microtubules ${ }^{56,52}$.

Importantly, the proline-rich region is the target of serine and threonine kinases, which have been connected to $A D^{110-112}$. Amniai et al. reported that phosphorylation of the T231in the P2 domain, inhibits tau's ability to induce tubulin polymerization ${ }^{48}$. Phosphorylated T231 is found in the cerebrospinal fluid (CSF) of patients, which were affected by dementia and who further developed $A D^{113,114}$, and is related to early stages of neurodegeneration ${ }^{115}$. NMR studies on the structural impact of phosphorylation of T231 showed that this residue forms a salt bridge with the preceding R230, potentially interfering with the formation of intermolecular contacts with soluble tubulin ${ }^{49}$. The understanding of the physiological activity of tau, especially regarding the regulation of the cytoskeleton structure, represents a key step for the progress of research in AD.

Hernández-Vega and collaborators recently reported that tubulin is recruited into tau droplets and can polymerize from them into microtubules ${ }^{71}$ (schematically represented in Figure 1.9). Tau droplets, formed in the presence of dextran, deformed right after the addition of tubulin and GTP as the microtubules formed. The authors proposed that the droplets, where tau concentration is increased, might act as nucleating centers for the organization of the microtubules within the axons, where no MTOC are present.

\subsubsection{The role of charges in tau-tubulin/microtubule interaction}

The tau protein is mainly positively charged due to the presence of many lysines and other positively charged residues (Figure 1.8 , section 1.2 .2 ). On the other side, microtubules have a negative net charge. Thus, electrostatic interactions play an important role for the interaction of tau with tubulin and with microtubules: it has been reported that the Cterminal tail of tubulin provides important contribution to the tau/tubulin interaction due to its negative charge ${ }^{116,117}$. Post-translational modifications of tau, which alter the charge distribution, can impair its interaction with microtubules and/or with soluble tubulin. The 
microtubule affinity-regulating kinase (MARK) 2 mediates phosphorylation on serine residues within the pseudo-repeat region, which has drastic effects on the affinity of tau to microtubules ${ }^{90,118}$. In addition, phosphorylation ofT231 in the proline-rich region, impaired tau's ability to polymerize tubulinbut did not affect its binding to microtubules ${ }^{48}$. Acetylation of lysine residues also decreases the amount of positive charge in the sequence, negatively affecting tau's ability to bind microtubules ${ }^{119}$.

\subsubsection{Structural insights into the binding of tau to tubulin and microtubules}

Due to the important role of tau bindingto and promoting tubulin polymerization, and the pathological consequences of the loss of such functions, the structure of tau in complex with tubulin and microtubules is a long thought aim. Indeed, several models for the interaction between tau and tubulin/microtubules have been proposed $98,100,101,120$.

As a consequence of its intrinsically disordered character, tau binds to microtubules in a dynamic fashion ${ }^{99}$. NMR studies showed that tau contact with microtubules occurs at the interface of $\alpha$ - $\beta$-tubulin heterodimers ${ }^{98}$. In complex with microtubules, a locally stable structure is formed in tau, in which the PGGG motifs at the end of the R1 and the R2 acquire a hairpin-like conformation ${ }^{100}$. Using single-molecule Förster resonance energy transfer, it was also observed that the tau repeats expand upon binding to soluble tubulin and long-range contacts between both termini and the microtubule-binding region are diminished ${ }^{121}$.

Cryo-EM in combination with molecular modeling (using the modeling software Rosetta) has also been used to obtain structural information about the microtubulebound state of tau. Kellogg and co-workers characterized the structure of synthetic tau constructs, which comprise four copies of either R1 or R2, respectively, in complex with microtubules ${ }^{101}$. In the structural model, the repeat domains are positioned on the tubulin protofilaments and interact in tandem with the microtubule surface (Figure 1.14). 

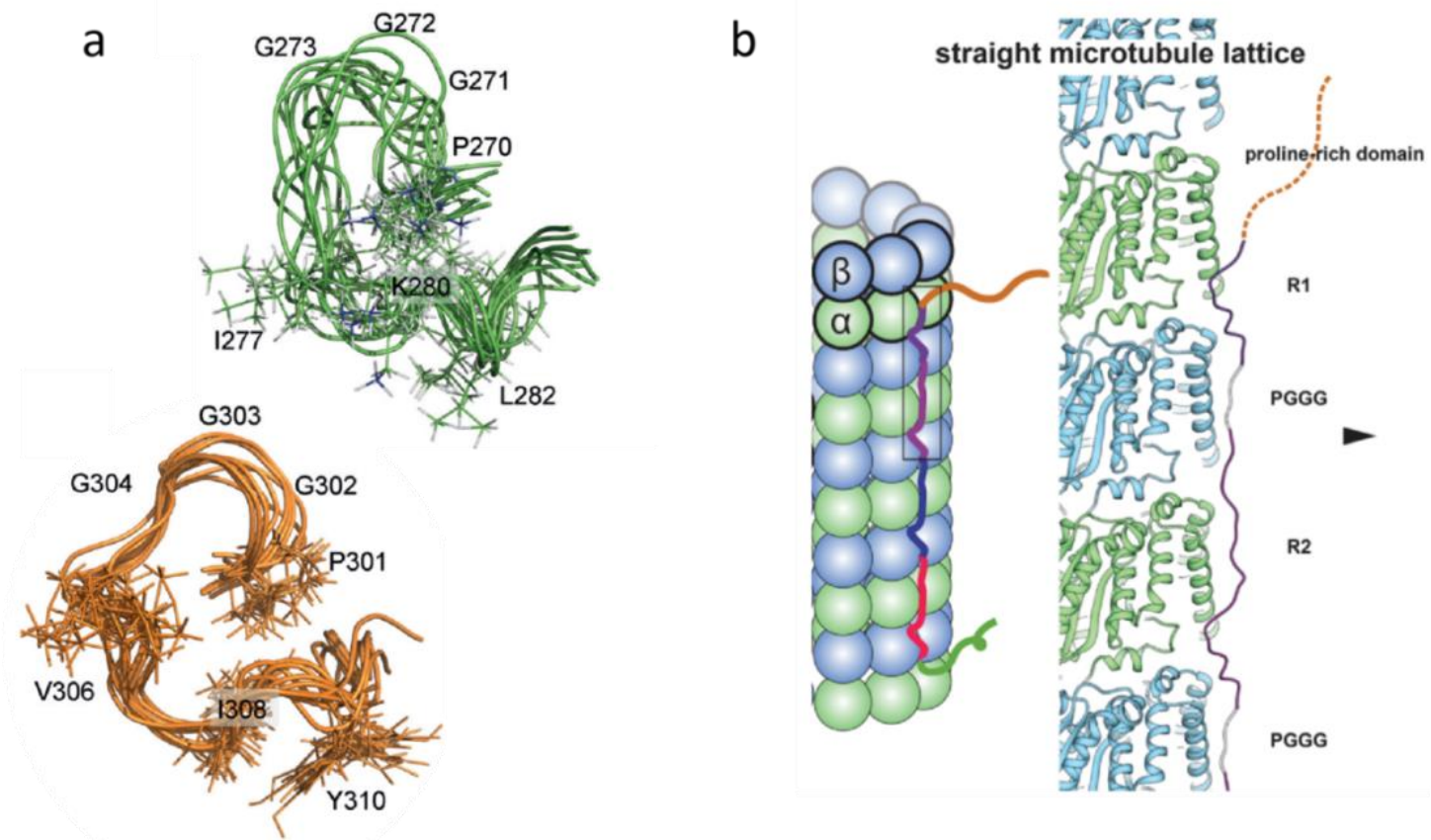

Figure 1.14. Modelling of tau bound to the microtubule surface. a) The PGGG motifs at the end of the R1 (green structure) and R2 (orange structure) adopt a hairpin fold when tau is bound to microtubules. Taken from Kadavath et al.,Angew. Chem. Int. Ed. 2015. b) Tau R1x4 and R2x4 synthetic constructs were used to obtain a three-dimensional cryo-EM model of tau's repeat region bound to microtubules. In this model, the repeats bind at the interface between $\alpha \beta$-tubulin heterodimers. The PGGG motifs at the end of each pseudo-repeatas well as the proline-rich regioncould not be identified in the electron density. Taken from Kellogg et al., Science 2018.

Due to their sequence homology, the pseudo-repeats are supposed to bind in a modular fashion. In particular, S262, conserved in the KXGS motif, was suggested to form a hydrogen bond with E434 of $\alpha$-tubulin in the cryo-EM/Rosetta model. This observation might explain why the phosphorylation of this residue has a negative impact on the binding to microtubules. However, the structure of the proline-rich region could not be defined in the model, despite its relevance for binding to microtubules and soluble tubulin. In addition, the PGGG motif at the end of each pseudo-repeat as well as the Cterminal tail remained unresolved in this model ${ }^{101}$. 


\subsection{Tau phosphorylation}

Due to the presence of hyperphosphorylated tau in $\mathrm{PHFs}^{122}$, many studies have focused on the molecular and cellular consequences of tau phosphorylation ${ }^{48,115,123-126}$. In addition, increasing evidence shows that other post-translational modifications (PTMs) contribute to the regulation of tau's physiological activityas well as to its pathological aggregation ${ }^{89}$.

Monoclonal antibodies recognize phosphorylated epitopes in PHFs in AD brain: the antibody AT8 recognizes phosphorylated S199, S202,T205 ${ }^{127,128}$ and S208 ${ }^{129}$; the antibody AT180 recognizes phosphorylated T231 and S235 ${ }^{130}$; the antibodies AT100 and PHF1 identify the epitopes pT212/pS214 and pS396/pS404, respectively ${ }^{48}$. The tau sequence is rich in serine (45), threonine (35) and tyrosine (5) residues, which make it an ideal target for many kinases ${ }^{26}$. Proline-directed protein kinases (PDPK) phosphorylate serine and threonine residues in the proline-rich region ${ }^{16,23}$. Non-proline-directed kinases (nonPDPK) are responsible for the phosphorylation of serines and tyrosines in the pseudorepeats region and at the $\mathrm{C}$-terminus ${ }^{23}$. Some of them are described in the following sections but for a more exhaustive reading the review from Martin et al. is recommended ${ }^{23}$. Kinases are identified as key players in the aberrant phosphorylation of tau and are considered promising candidates for therapeutic treatments, but because so many kinases are involved in tau phosphorylation, finding the correct target is challenging ${ }^{131}$. On top of that, the same kinases which are acting on tau phosphorylate many other proteins, so modulating their activity often causes side effects.

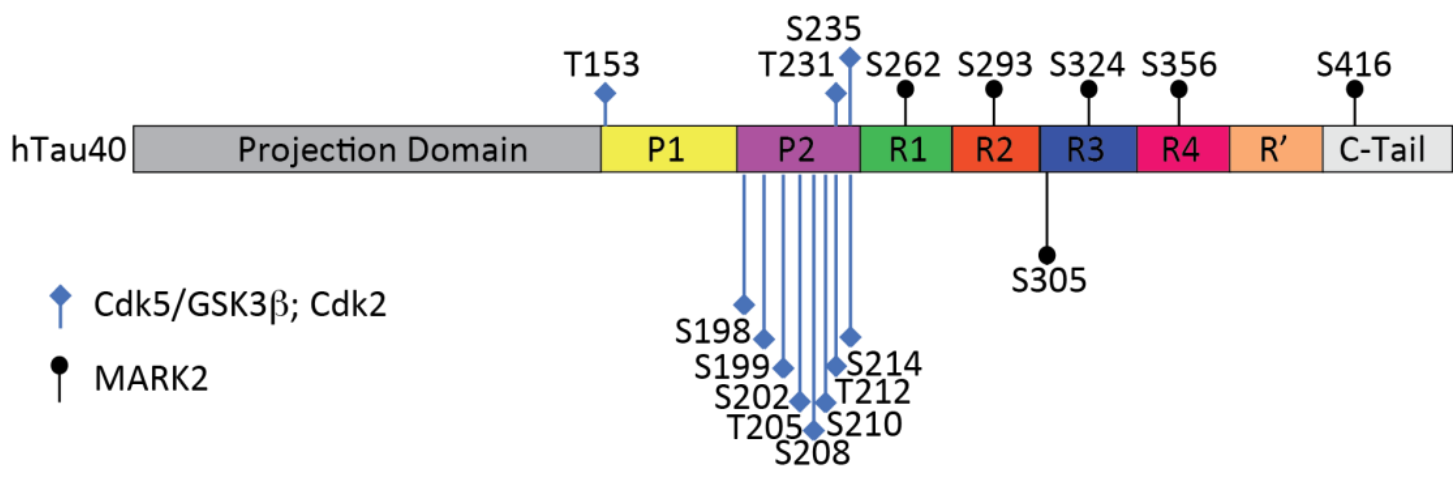

Figure 1.15. Tau residues phosphorylated by Cdk2, Cdk5/GSK3 $\beta$ and MARK2. Schematic representation of tau domain organization and the sites of phosphorylation in the proline-rich region, mediated by Cdk2 or Cdk5/GSK3 $\beta$ (blue marks), and in the MTBR, mediated by MARK2 (black circles) based on Martin et al., Ageing Res Rev, 2013. 


\subsubsection{Tau phosphorylation mediated by PDPK}

PDPKs add a phosphate group to serine or threonine preceding a proline residue (Ser/ThrPro motif). They target primarily tau's proline-rich region, which is rich in serine and threonines residues.

Among these, the cyclin dependent kinase (Cdk) 5 and the glycogen synthase kinase (GSK) $3 \beta$ act in a sequential manner to phosphorylate tau in the proline-rich region $22,23,132$. As reviewed by Martin et al. (2013), the major sites reported to be phosphorylated in vitro by the combination of Cdk5 and GSK3 $\beta$ are S202, T205, T231, S235, S396 and S404. These phosphorylated residues can be recognized by the AT8, AT180, AT100 and PHF-1 monoclonal antibodies (see section 1.6).

Cdk5 is a pivotal regulator of several signaling pathways during neuronal development. It is also linked to abnormal phosphorylation of tau, e.g. residues found phosphorylated in tau fibrils are targets of this kinase, and its co-activator p25 is found overexpressed in AD brain ${ }^{132}$. Cdk5 priming phosphorylation on $\mathrm{S} 235$ is necessary for the phosphorylation of T231 mediated by GSK3 $\beta^{112,133}$.

GSK3 $\beta$ is an important regulator of neuronal growth and development through its involvement in several signaling pathways ${ }^{134}$. Recent findings, linked GSK3 $\beta$ to the regulation of tau alternative splicing by colocalization in nuclear speckles and phosphorylation of the SC35 protein ${ }^{111,135}$. In AD brain, approximately 29 of the phosphorylated amino acid residues are targeted by GSK3 $\beta^{89}$. On top of that, the kinase colocalizes with tau fibrils together with $\mathrm{Cdk}^{136}$, indicating a tight correlation with the disease. Tau phosphorylation in vitro is also mediated by the cyclin dependent kinase 2 $(C d k 2)^{22,48,130}$. Unlike $C d k 5$, no correlations with AD have been reported for Cdk2. This kinase is responsible for the cell cycle regulation and is not present at high concentrations in mature neurons ${ }^{22}$. Nevertheless, Cdk2 alone produces the AT180 epitope without the need of additional kinases, which makes this enzyme usefull for in vitro studies ${ }^{48,130}$. 


\subsubsection{Tau phosphorylation mediated by non-PDPKs}

Several tau-relevant kinases belong to this group, e.g. tau-tubulin kinases $1 / 2$ (TTBK1/2), the microtubule affinity-regulating kinase (MARK), the $\mathrm{Ca}^{2+} /$ calmodulin-dependent protein kinase II (CaMKII), C-abl and many others. The TTBK1/2 kinases are involved in the phosphorylation of tubulin and tau and are reported to enhance tau aggregation into fibrils ${ }^{23}$. Mice models overexpressing these kinases display tau aggregation as well as an enhanced activation of Cdk5 and GSK3 $\beta$, suggesting an interplay of different kinases in $A D^{23}$. This group of kinases is involved in the regulation of microtubule rearrangement, important during neuronal growth, cellular polarity and neuron migration ${ }^{137}$. The family of microtubule affinity-regulating kinases (MARK 1-4) isinvolved in the formationof axons and the regulation of tau's affinity to microtubules. Target sites are S262, S293, S324 and S356, which are located in the KXGS consensus motifs in the $R 1, R 2, R 3$ and $R 4$ repeats, respectively ${ }^{23,118}$. Tau fibrils in AD brains are phosphorylated on S262 and S356, which links MARK2 to pathological events ${ }^{90}$. Other nonPDPKs involved in tau phosphorylation are CaMKII and C-Abl. CaMKII phosphorylates threonine and serine residues in the tau sequence, some of which are found in AD brains ${ }^{23}$. CAbl belongs to the family of the non-receptor tyrosine kinases, it is a ubiquitously expressed protein, highly active during cell differentiation and involved in cytoskeleton remodeling and neuronal differentiation ${ }^{138}$. This kinase phosphorylates residues $\mathrm{Y} 18$ ( $\mathrm{N}$-terminal domain), Y197 (PRR), Y310 (R3) and Y394 ( $R^{\prime}$ ) and it is known to phosphorylate the fetal isoform of tau, htau23, during neuronal development ${ }^{139}$. In a normal adult brain, C-Abl is quiescent but an aberrant activation has been linked to $A D^{140}$ : phosphorylation activated forms of $C-A b l$ and tyrosine-phosphorylated forms of tau have been co-stained in PHFs of AD brain and other tau inclusions ${ }^{141}$.It has been suggested that phosphorylation on $\mathrm{Y} 18$ and $\mathrm{Y} 394$ could be relevant for tau fibril formation ${ }^{138}$. 


\subsection{Aims of this work}

Tau is the object of intense research due to its intimate connection with several neurodegenerative disorders ${ }^{35-38}$. Recent cryo-EM analysisprovided structural insights into tau fibrils purified from the brains of patients with different tauopathies. In addition, NMR spectroscopy and cryo-EM have started to characterize the structural details of the tau/microtubule interaction $79,80,98,100,101$. From these studies, increasing information is available for the structural properties of the pseudo-repeats in complex with microtubules and within amyloid fibrils. In contrast, little is known about the structure of the proline-rich region in these two states. The proline-rich region plays an important role in establishing protein-protein interactions and is involved in the polymerization of tubulin into microtubules. In addition, its high content of serine and threonine residues, makes the proline-rich region a target of numerous kinases, which act on tau in physiological and pathological conditions. Because hyperphosphorylated tau is linked to a decreased affinity to microtubules as well as an increased propensity to form fibrils, a deeper knowledge of the roles played by the proline-rich region is necessary to better understand these events ${ }^{90,118}$.

The focus of this work is on tackling the involvement of the proline-rich region in the microtubule polymerization process and in its contribution to the formation of tau fibrils in vitro and is divided into two projects.

In the first part, in vitro aggregated amyloid fibrils of tau constructs containing both the proline-rich region and the pseudo-repeats are investigated using sSNMR.

In the second part, the molecular mechanism of the interaction between the proline-rich region and soluble tubulin/microtubules is studied. Using solution-state NMR, LLPS assays, differential interference contrast (DIC) and fluorescence microscopy the effect of phosphorylation in the proline-rich region is addressed. 


\section{Materials and methods}

\subsection{Preparation of K32: protein expression and purification}

The K32 construct comprises the P2 region (residues S198-Q244) and the complete pseudo-repeat domain, including repeats R1, R2, R3, R4 and R' (residues Q244-S400) of full-length htau40 and is schematically represented in the introduction section, figure 1.7. The plasmid carrying the K32 coding sequence was kindly provided by the laboratory of Prof. Dr. Eckhard Mandelkow.

\subsubsection{E. coli transformation}

XL2Blue ultracompetent cells (Agilent technologies) were used to store the plasmid. BL21 ${ }^{\mathrm{TM}}$ DE3 competent cells (Novagen) were used for protein overexpression. $25 \mu$ of cells stored at $-80{ }^{\circ} \mathrm{C}$ were thawed on ice for 25 minutes. Subsequently, $1 \mu$ of each DNA sample was added to the cells and equilibrated for 30 minutes. Heat-shock transformation was performed for 30 seconds at $42^{\circ} \mathrm{C}$ on a thermo block, cells were afterwards put back on ice. Cells were incubated without antibiotics for one hour after addition of $1 \mathrm{~mL} \mathrm{LB}$ medium, then harvested by centrifugation at $4000 \mathrm{rpm}$ for five minutes. Subsequently, $50 \mu \mathrm{l}$ of the harvested cells were streaked onto LB-agar plates supplemented with $5 \mathrm{mg} / \mathrm{ml}$ kanamycin and cloramphenicol, respectively. Plates were incubated overnight at $37^{\circ} \mathrm{C}$.

\subsubsection{Protein expression}

Quantities refer to protein purification from $1 \mathrm{~L}$ of LB. BL21 ${ }^{\mathrm{TM}}$ (DE3) E. coli cells carrying the $\mathrm{K} 32$ coding sequence were grown in $25 \mathrm{ml} \mathrm{LB}$ medium containing kanamycin antibiotic overnight. This volume was transferred to 1 L LB medium and allowed to grow until an $\mathrm{OD}_{600}$ of 0.7 was reached. Protein expression under the T7 promoter was induced with IPTG at a final concentration of $0.5 \mathrm{mM}$. Cells were harvested after 3 hours of induction by centrifugation at $7500 \mathrm{rpm}$ in a JLA-8.1 rotor in a Beckman Coulter Avanti centrifuge for 30 minutes. Cell pelletswerestored at $-20{ }^{\circ} \mathrm{C}$ for further purification. 


\subsubsection{Purification protocols and procedure}

The pellet of $\mathrm{K} 32$, harvested from $1 \mathrm{~L}$ of cell culture, was resuspended in $35 \mathrm{~mL}$ lysis buffer, with the following composition:

$\begin{array}{ll}20 \mathrm{mM} & \text { MES, pH } 6.8 \\ 1 \mathrm{mM} & \text { EGTA } \\ 625 \mu \mathrm{l} & \text { Lysozyme }(100 \mathrm{mg} / \mathrm{ml} \text { stock) } \\ 625 \mu \mathrm{l} & \text { DNase I }(100 \mathrm{mg} / \mathrm{ml} \text { stock) } \\ 1 \quad \text { Tablet } & \text { EDTA-free Protease inhibitor } \\ 1.0 \mathrm{mM} & \text { PMSF } \\ 5.0 \mathrm{mM} & \mathrm{DTT} \\ 0.2 \mathrm{mM} & \mathrm{MgCl}_{2}\end{array}$

After complete resuspension of the pellet in the lysis buffer, cell membrane disruption was achieved using a French press (Emulsiflex-C3). Sodium chloride was added to the solution to reach a final concentration of $500 \mathrm{mM}$. Samples were boiled in a water bath at $98^{\circ} \mathrm{C}$ for 20 minutes. Sedimentation of insoluble particles and precipitated protein was achieved by ultra-centrifugation in a $45 \mathrm{Ti}$ Ultra Rotor at $127,000 \mathrm{~g}$ for 30 minutes in Beckman Coulter centrifuge (Optima XPN 80). $20 \mathrm{mg} / \mathrm{ml}$ of streptomycin sulfate (Carl Roth $\left.^{\circledR}\right)$ were added to the supernatant to induce nucleic acid precipitation ${ }^{142}$. After incubation for 15 minutes the samples were centrifuged (Eppendorf Centrifuge, $5810 \mathrm{R}$ ) at $15,000 \mathrm{~g}$ at $4{ }^{\circ} \mathrm{C}$ for 30 minutes. $0.361 \mathrm{~g} / \mathrm{ml}$ of ammonium sulfate (Carl Roth ${ }^{\circledR}$ ) were added to the retrieved supernatant to induce $\mathrm{K} 32$ precipitation ${ }^{143}$. Another cycle of incubation (15 minutes) and centrifugation $\left(15,000 \mathrm{~g}\right.$ at $4{ }^{\circ} \mathrm{C}$ for 30 minutes) followed this procedure. The pellet was resuspended in buffer and dialysed overnight against the buffer prepared as follows:

$\begin{array}{ll}20 \mathrm{mM} & \mathrm{MES}, \mathrm{pH} 6.8 \\ 150 \mathrm{mM} & \mathrm{NaCl} \\ 1.0 \mathrm{mM} & \text { EDTA } \\ 2.0 \mathrm{mM} & \text { DTT } \\ 0.1 \mathrm{mM} & \text { PMSF }\end{array}$

After dialysis, samples were loaded on a MonoS $^{\circledR} 10 / 100 \mathrm{GL}$ column for ion-exchange chromatography. The protein elution resulted from a combination of Buffer A and B achieved with a gradient between 0 and $60 \%$ over 15 column volumes. 
The buffers presented the following composition:

$\begin{array}{llll}\text { Buffer A } & & \text { Buffer B } & \\ 20 \mathrm{mM} & \mathrm{MES}, \mathrm{pH} 6.8 & 20 \mathrm{mM} & \mathrm{MES}, \mathrm{pH} 6.8 \\ 150 \mathrm{mM} & \mathrm{NaCl} & 1.0 \mathrm{M} & \mathrm{NaCl} \\ 1.0 \mathrm{mM} & \text { EDTA } & 1.0 \mathrm{mM} & \text { EDTA } \\ 2.0 \mathrm{mM} & \text { DTT } & 2.0 \mathrm{mM} & \text { DTT } \\ 0.1 \mathrm{mM} & \text { PMSF } & 0.1 \mathrm{mM} & \text { PMSF }\end{array}$

To achieve maximal purity of the sample, size exclusion chromatography was performed. K32 sample was concentrated up to a volume of $1 \mathrm{ml}$ and loaded on a HiLoad ${ }^{\text {TM }}$ 26/200 Superdex 75 pg (GE Healthcare Life Sciences). The buffer for the size exclusion chromatography was prepared as follows:

$\begin{array}{ll}\text { PBS } & \text { pH 7.4 } \\ 500 \mathrm{mM} & \mathrm{NaCl} \\ 1.0 \mathrm{mM} & \text { DTT }\end{array}$

\subsection{P2R2 and P2R3 peptides preparation}

\subsubsection{Peptide sequence design}

The peptides $\mathrm{P} 2 \mathrm{R} 2$ and $\mathrm{P} 2 \mathrm{R} 3$ combine the amino acid sequences of the P2 domain (residues S198-Q244) with either the R2 domain (residues V275-S305) or the R3 domain (residues V306-Q336) of htau40, respectively (Figure 2.1). Plasmids encoding the DNA sequence of either P2R2 or P2R3 were purchased from GeneArt ${ }^{\circledR}$ gene synthesis from Invitrogen, Thermo Fisher Scientific. The two native cysteine residues C291 and C322 of htau40, which are located in the R2 and R3 domains respectively, were replaced by alanine in the P2R2 and P2R3 peptides. Codon optimization of the DNA sequence for the E. coli expressing system was also included in the requirements of the designed plasmids. 
a

\begin{tabular}{l|l|l|l|l|l|l|l|l|l|} 
hTau40 & Projection Domain & P1 & P2 & R1 & R2 & R3 & R4 & $R^{\prime}$ & C-Tail \\
\cline { 2 - 6 }
\end{tabular}

P2R2

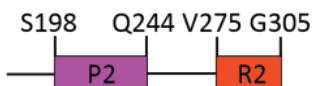

P2R3

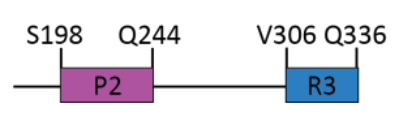

b P2R2 ${ }^{1985 S P G S P G T P G R S R T P S L P T P ~ P T R E P K K V A V V R T P P K S P S S A K S R L Q ~} 244275$ VQIINKKLDLSNVQSKCTGSKDNIKHVPGGGS ${ }^{205}$ A

P2R3 198SSPGSPGTPGRSRTPSLPTP PTREPKKVAVVRTPPKSPSSAKSRLQ244306VOIVYKPVDLSKVTSKCGGSLGNIHHKPGGGQ 337

Figure 2.1. $P 2 R 2$ and $P 2 R 3$ domain organization and sequence. a) The domain organization of the peptides P2R2 and P2R3 is represented together with that of htau40. The residues delimiting the domains combined in P2R2 and P2R3 are indicated. b) The sequences of P2R2 and P2R3 comprise the P2 domain and either the $\mathrm{R} 2$ or the R3 sequence.The substitution of $\mathrm{C} 291 / \mathrm{C} 322$ to alanine is indicated for each peptide.

\subsubsection{PCR}

Prior to cloning, P2R2 and P2R3 optimized sequences were screened for restriction enzyme type I and II using the online NEB cutter V 2.0 (http://www.labtools.us/nebcutter$\mathrm{v} 2-0 /)$ tool to avoid restriction of the coding sequences. After this, sequences were amplified using PCR. Primers for the reaction were designed in order to allow future cloning of the inserts into the pET28a plasmid vector between BamHI and HindIII restriction sites. For P2R2, the following forward (F) and reverse $(R)$ primers where used:

$$
\begin{array}{ll}
\text { F 5'-AATTGGATCCAGCAGTCCGGGTTCTCCG-3' } & \text { (BamHI) } \\
\text { R 5'-CCCAAGCTTCTAGCTACCACCACCCGGAAC-3' } & \text { (HindIII) }
\end{array}
$$

For the P2R3 peptide the following forward $(F)$ and reverse $(R)$ primers where used:

$$
\begin{array}{ll}
\text { F 5'-AATTGGATCCAGCAGTCCGGGTTCTCCG-3' } & \text { (BamHI) } \\
\text { R 5'-CCCAAGCTTCTACTGACCACCACCCGGTTT-3' } & \text { (HindIII) }
\end{array}
$$

Lyophilized primers where ordered from Sigma-Aldrich Chemie GmbH. 
Each PCR reaction mixture was prepared as follows:

$\begin{array}{ll}22.5 \mu \mathrm{l} & \mathrm{ddH}_{2} \mathrm{O} \\ 0.5 \mu \mathrm{l} & \text { DMSO } \\ 0.5 \mu \mathrm{l} & \text { Forward primer } \\ 0.5 \mu \mathrm{l} & \text { Reverse Primer } \\ 25.0 \mu \mathrm{l} & \text { Phusion Polymerase (New England Biolabs }{ }^{\circledR} \text { ) } \\ 1.0 \mu \mathrm{l} & \text { Templates } \\ 50 \mu \mathrm{l} & \text { Total Volume }\end{array}$

The following PCR cycles were used:

$\begin{array}{ll}\text { Double strand denaturation } & 98^{\circ} \mathrm{C} \text { for } 1 \mathrm{~min} \\ \text { Primer annealing } & 83^{\circ} \mathrm{C} \text { for } 30 \mathrm{~s} \\ \text { Elongation } & 72{ }^{\circ} \mathrm{C} \text { for } 10 \mathrm{~min} \\ \text { Cycles repetition } & 32 \text { times } \\ \text { Storage of the amplified sequences } & 4{ }^{\circ} \mathrm{C}\end{array}$

The successful outcome of the PCR amplification was controlled by agarose gel. Plasmids were purified from gel using the PCR-clean up ${ }^{\circledR}$ kit from MACHEREY-NAGEL.

\subsubsection{Restriction enzyme double digestion and ligation reaction}

$P 2 R 2$ and P2R3 optimized sequences were cloned into the $p E T 28 a$ vector between BamHI and HindIII restriction sites. The expression vector had an additional $Z$ domain of staphylococcal protein A, expressed as protein fused to either P2R2 or P2R3, and a TEV consensus motif for cleavage of His-tag sequences. The PET28 vector and DNA sequences of either P2R2 or P2R3, were incubated with BamHI and HindllI restriction enzimes (New England Biolabs $\left.{ }^{\circledR}\right)$. For the restriction reaction of pET28 the following mixture was used:

$\begin{array}{ll}10 \mu \mathrm{l} & \text { pET28_Z2 } \\ 1 \mu \mathrm{l} & \text { BamHI } \\ 1 \mu \mathrm{l} & \text { HindIII } \\ 5 \mu \mathrm{l} & \text { CutSmart }^{\circledR} \text { buffer (New England Biolabs } \\ & { }^{\circledR} \text { ) } \\ 33 \mu \mathrm{l} & \text { dd }_{2} \mathrm{O} \text { (for the purpose autoclaved) } \\ 50 \mu \mathrm{l} & \text { Total volume }\end{array}$


The incubation time was two hours at $37^{\circ} \mathrm{C}$. After one hour and $30 \mathrm{~min}$ of incubation, alkaline phosphatase (CIP, New England Biolabs ${ }^{\circledR}$ ) was added to the mixture to dephosphorylate the $5^{\prime}$ and $3^{\prime}$ ends. For P2R2 and P2R3 inserts, the following mixture was used:

$\begin{array}{lll}35 \mu \mathrm{l} & \text { Insert } \\ 1 \mu \mathrm{l} & \text { BamHI } \\ 1 \mu \mathrm{l} & \text { HindIII } \\ 5 \mu \mathrm{l} & \text { CutSmart }^{\circledR} \text { buffer } \\ 8 \mu \mathrm{l} & \text { dd }_{2} \mathrm{O} \text { (for the purpose autoclaved) } \\ 50 \mu \mathrm{l} & \text { Total volume }\end{array}$

Reactions were incubated for twohours at $37^{\circ} \mathrm{C}$.

The ligation was performed in a water bath at $16{ }^{\circ} \mathrm{C}$ overnight. The required amount of expression vector and insert was calculated according to:

$$
1: a=\frac{\mu g \text { vector }}{b p \text { vector }}: \frac{\mu g \text { insert }}{b p \text { insert }}
$$

Where 1:a represents the ratio between the amount of vector to use and "a" is the amount of insert, $b p$ is the length in base pairs of the expression vector and the insert, respectively.

A reaction mixture for 3 -fold excess of insert, was used:

$0.09 \mu \mathrm{l} \quad$ Insert

2.23 $\mu \mathrm{l} \quad$ Expression vector

$1.0 \mu \mathrm{l} \quad$ T4 DNA Ligase (New England Biolabs ${ }^{\circledR}$ )

$1.0 \mu \mathrm{l} \quad$ T4 DNA Ligase reaction buffer (New England Biolabs ${ }^{\circledR}$ )

$5.68 \mu \mathrm{l} \quad \mathrm{ddH}_{2} \mathrm{O}$ (for the purpose autoclaved)

$10 \mu \mathrm{l} \quad$ Total volume

After ligation, Rosetta 2 (DE3) E. coli cells were transformed and streaked on LB agarose plates supplemented with $5 \mathrm{mg} / \mathrm{ml}$ of kanamycin. DNA extracted from positive clones was sequenced by Microsynth SEQLAB.

\subsubsection{E. coli transformation}

XL2Blue ultracompetent cells (Agilent technologies) were used for amplification of the expression vector and Rosetta ${ }^{\mathrm{TM}} 2$ (DE3) Singles ${ }^{\mathrm{TM}}$ competent cells (Novagen) were used 
for protein overexpression. The protocol for cell transformation is reported in section 2.1.4.

\subsubsection{Protein expression}

Protein expression and purification protocols were optimized for both P2R2 and P2R3 peptides. Protein amount and buffer solutions refer to protein purification from 1L of LB. Rosetta $^{\mathrm{TM}} 2$ (DE3) Singles ${ }^{\mathrm{TM}}$ containing $\mathrm{pET} 28$ a plasmid carrying either P2R2 or P2R3 sequences were grown overnight in $25 \mathrm{ml}$ LB medium, supplemented with $5 \mathrm{mg} / \mathrm{ml}$ of kanamycin and cloramphenicol. The starter culture was transferred to 1 L LB medium and allowed to grow until an $\mathrm{OD}_{600}$ of 0.7 was reached. Protein expression was induced with a final concentration $0.5 \mathrm{mM}$ IPTG. Cells were harvested after 4 hours of induction by centrifugation at $7500 \mathrm{rpm}$ in a JLA-8.1 rotor in a Beckman Coulter Avanti centrifuge for 30 minutes. Cell pellets were stored at $-20^{\circ} \mathrm{C}$ for further purification.

\subsubsection{Purification protocols and procedure}

Pellets of the P2R2 and P2R3 peptides were resuspended in $40 \mathrm{~mL}$ lysis buffer:

$\begin{array}{ll}20 \mathrm{mM} & \text { Tris-HCL, } \mathrm{pH} 8 \\ 500 \mathrm{mM} & \mathrm{NaCl} \\ 500 \mu \mathrm{l} & \text { Lysozyme (100 mg/ml stock) } \\ 1 \text { Tablet } & \left.\text { EDTA-free Protease inhibitor mix (Roche }{ }^{\circledR}\right) \\ 250 \mu \mathrm{l} & \text { DNase }(1 \mathrm{mg} / \mathrm{ml} \text { final concentration) } \\ 5 \mathrm{mM} & \text { DTT } \\ 0.2 \mathrm{mM} & \mathrm{MgCl}_{2}\end{array}$

Subsequently, cell membranes were disrupted by sonification cycles of 5 minutes using $30 \%$ power and 5 minutes at $60 \%$ power with 30 seconds of pause between each pulse (SONOPULS Ultraschall, Bandelin). Sedimentation of insoluble particles was achieved by centrifugation at 20,000 rpm for 30 minutes in a JA 25.50 in a Beckman Coulter Avanti centrifuge. The purification steps were performed using Qiagen Ni-NTA Agarose for gravity-flow chromatography. The resin beads were washed with water, packed into a Pierce $^{\mathrm{TM}}$ disposable column of $10 \mathrm{ml}$ and equilibrated with lysis Buffer prior use, following the manual (The QIAexpressionist, from QIAGEN). The supernatant from the 
centrifugation of sonicated cells was further incubated for 30 minutes with Ni-NTA Agarose at $4{ }^{\circ} \mathrm{C}$ to allow interaction of the His-tagged protein with the nickel ions in the agarose. Washing steps were performed with a buffer containing increasing concentration of imidazole $(20 \mathrm{mM}$ Tris- $\mathrm{HCL}, \mathrm{pH} 8,500 \mathrm{mM} \mathrm{NaCl}$, and 10 to $50 \mathrm{mM}$ imidazole) in order to remove impurities and non-specific binding products. Proteins were eluted from the Ni-NTA Agarose with an elution buffer containing $20 \mathrm{mM}$ Tris-HCL, pH8, $500 \mathrm{mM} \mathrm{NaCl}, 200 \mathrm{mM}$ imidazole. An SDS-PAGE gel sample was prepared for each washing step and elution fraction. To remove the His-tag from the eluted protein, $0.5 \mathrm{mg}$ of TEV protease was added. Subsequently, samples were transferred to dialysis membranes (8-10 kDa cut-off) and incubated overnight at $4{ }^{\circ} \mathrm{C}$ in a TEV buffer, prepared as follows:

$20 \mathrm{mM}$ Tris-HCL, $\mathrm{pH} 8$

$500 \mathrm{mM} \quad \mathrm{NaCl}$

$0.3 \mathrm{mM}$ EDTA

After the removal of the His-Tag, a second cycle of gravity-flow chromatography using Ni-NTA agarose was performed. P2R2 and P2R3 eluted in the first washing step as flow through.

To achieve maximal purity of the sample an additional size exclusion chromatography step was included into the protocol. P2R2 and P2R3 samples were concentrated up to a volume of $2 \mathrm{ml}$ and loaded on a HiLoad ${ }^{\mathrm{TM}}$ 16/200 Superdex $75 \mathrm{pg}$ (GE, Healthcare Life Sciences). The buffer for the size exclusion chromatography was prepared as follows:

$20 \mathrm{mM}$ Tris- $\mathrm{HCl}, \mathrm{pH} 8$

$250 \mathrm{mM} \quad \mathrm{NaCl}$

$1 \mathrm{mM}$ EDTA

\subsection{Uniform isotope labeling of proteins for solution and solid- state NMR}

In order to label P2R2, P2R3 and K32 uniformly with ${ }^{15} \mathrm{~N}$ and ${ }^{13} \mathrm{C}$ isotopes, the protocols described in sections 2.1.5 and 2.2 were used, substituting the LB medium for M9-minimal medium. A $1 \mathrm{~L}$ of culture with M9 medium composition is described as follows: 


\begin{tabular}{|c|c|}
\hline $200 \mathrm{ml}$ & $5 x$ M9 salts * \\
\hline $2.0 \mathrm{ml}$ & $\mathrm{MgSO}_{4} 1 \mathrm{M}$ \\
\hline $1.0 \mathrm{ml}$ & $\mathrm{CaCl}_{2} \quad 1 \mathrm{M}$ \\
\hline $6.0 \mathrm{ml}$ & Thiamine $(5 \mathrm{mg} / \mathrm{ml})$ \\
\hline $10 \mathrm{ml}$ & $100 x$ Trace elements $* *$ \\
\hline $1 \mathrm{~g}$ & ${ }^{15} \mathrm{NH}_{4} \mathrm{Cl}$ \\
\hline $2 \mathrm{~g}$ & ${ }^{13} \mathrm{C}$-glucose \\
\hline $880 \mathrm{ml}$ & $\mathrm{dd}_{2} \mathrm{O}$ \\
\hline \multicolumn{2}{|c|}{ * $5 x \mathrm{M} 9$ salts } \\
\hline $16.95 \mathrm{~g}$ & $\mathrm{Na}_{2} \mathrm{HPO}_{4}$ \\
\hline $7.5 \mathrm{~g}$ & $\mathrm{KH}_{2} \mathrm{PO} 4$ \\
\hline $1.25 \mathrm{~g}$ & $\mathrm{NaCl}$ \\
\hline \multicolumn{2}{|c|}{ ** 100x Trace elements } \\
\hline $5 \quad g$ & EDTA \\
\hline 0.8 & $\mathrm{FeCl}_{3}$ \\
\hline $0.05 \mathrm{~g}$ & $\mathrm{ZnCl}_{2}$ \\
\hline $0.01 \mathrm{~g}$ & $\mathrm{CuCl}_{2}$ \\
\hline $0.01 \mathrm{~g}$ & $\mathrm{CoCl}_{2}$ \\
\hline $0.01 \mathrm{~g}$ & $\mathrm{H}_{3} \mathrm{BO}_{3}$ \\
\hline $1.6 \mathrm{mg}$ & $\mathrm{MnCl}_{2}$ \\
\hline
\end{tabular}

\subsection{Determination of protein concentration}

Because of the lack of tryptophans, the concentration of P2R2 (7 kDa) and P2R3 (7 kDa) was estimated using a sequence-specific extinction coefficient for $\operatorname{Abs}_{205} \mathrm{~nm}^{144}$. For P2R2 and P2R3 $\varepsilon_{205}$ was $225,230 \mathrm{M}^{-1} \mathrm{~cm}^{-1}$ and $235,710 \mathrm{M}^{-1} \mathrm{~cm}^{-1}$, respectively. The protein concentration was calculated using the Lambert-Beer law:

$$
c=A /(\varepsilon \cdot l)
$$

Where $A$ is the absorbance at $205 \mathrm{~nm}$, and $/$ is the pathlength of the quartz cuvette $(\mathrm{cm})$. The concentration of K32 (25 kDa) was also confirmed with bicinchoninic acid (BCA) Assay kit (Sigma-Aldrich). 


\subsection{Circular dichroism of P2R2 and P2R3 peptides in the monomeric form}

CD spectroscopy allows detection of secondary structure elements present in an optically active molecule, based on the deviation of polarized light that this can induce ${ }^{145}$. Measurements of protein CD in the far UV range $(260-190 \mathrm{~nm})$ is a useful tool to estimate secondary structure content, as each structural rearrangement is contributing with a typical absorption profile ${ }^{146}$. CD spectra of monomeric P2R2 and P2R3 peptides were acquired on a Chirascan (Applied Photophysics, UK) spectrometer. Samples in sodium phosphate buffer $50 \mathrm{mM}, \mathrm{pH} 6.8,50 \mathrm{mM} \mathrm{NaCl}$ were diluted in $\mathrm{ddH}_{2} \mathrm{O}$ to reach a final protein concentration of $25 \mu \mathrm{M}$. Measurements were performed in a $0.1 \mathrm{~cm}$ light path quartz cuvette.

General settings for the spectra acquisition were:

$\begin{array}{ll}\text { Temperature } & 20{ }^{\circ} \mathrm{C} \\ \text { Start wavelength } & 260 \mathrm{~nm} \\ \text { End wavelength } & 190 \mathrm{~nm} \\ \text { Scanning speed } & 100 \mathrm{~nm} / \mathrm{min} \\ \text { Bandwidth } & 1.0 \\ \text { Steps } & 0.1 \\ \text { Sampling (time-per-point) } & 0.5 \mathrm{~s} \\ \text { Repeats per spectrum } & 3\end{array}$

Baseline correction was performed subtracting the spectrum of the buffer acquired with the same parameter settings. Data are expressed as mean residue ellipticity (MRE) ${ }^{146}$ according to

$$
[\theta]=M R W \cdot \frac{\theta_{\lambda}}{10} \cdot d \cdot c
$$

Here, Mean Reside Weight (MRW) of P2R2 and P2R3 was $104.36 \pm 0.07, \theta_{\lambda}$ the observed ellipticity at $260 \mathrm{~nm}$ (in degrees), $d$ the pathlength $(\mathrm{cm})$ and $c$ the protein concentration $(\mathrm{g} / \mathrm{ml})^{c}$. The deconvolution of secondary structure information contained in the spectra was performed using the Dichroweb online calculation tool ${ }^{145}$. 


\subsection{Fibril formation and characterization}

\subsubsection{Fibrillization protocol}

The fibrillization of unlabeled and ${ }^{15} \mathrm{~N} /{ }^{13} \mathrm{C}$-labeled $\mathrm{P} 2 \mathrm{R} 2, \mathrm{P} 2 \mathrm{R} 3$ and $\mathrm{K} 32$ proteins was induced using heparin salt $\left(\mathrm{Mr}_{\mathrm{r}} 2 \times 10^{4} \mathrm{~g} / \mathrm{mol}, \operatorname{Carl~Roth}^{\circledR}\right)$ in a heparin-to-protein molar ratio of 1:4. Either P2R2 or P2R3 (200 $\mu \mathrm{M})$ were incubated with heparin $(50 \mu \mathrm{M})$ in a buffer containing Tris- $\mathrm{HCl} 25 \mathrm{mM}, \mathrm{pH} 7.4,0.02 \%$ sodium azide. In the case of $\mathrm{K} 32,100 \mu \mathrm{M}$ of final protein concentration were incubated with heparin $(25 \mu \mathrm{M})$ in a buffer containing Tris- $\mathrm{HCl} 25 \mathrm{mM}, \mathrm{pH} 7.4,0.02 \%$ sodium azide and $2 \mathrm{mM}$ DTT freshly added just before starting the reaction. Samples were incubated for three days at $37{ }^{\circ} \mathrm{C}$.During this time, ThT measurements of each sample were performed to monitor the fibrillization process. The reaction was then stopped by ultracentrifugation at $200000 \mathrm{~g}$, at $37^{\circ} \mathrm{C}$ for 1 hour in a TLA 100.3 rotor in a bench ultracentrifuge (Beckman Coulter, Optima MAX XP). The composition of the supernatant and the pellet was monitoredby SDS-PAGE.

\subsubsection{ThT fluorescence}

Fibril formation was constantly monitored performing a Thioflavin T (ThT) binding assay ${ }^{147}$. The measurements were carried on a 96 well plate in a Cary Eclipse fluorescence spectrophotometer with the following parameters:

$\begin{array}{ll}\text { Excitation wavelength } & 440 \mathrm{~nm} \\ \text { Emission wavelength } & 482 \mathrm{~nm} \\ \text { Emission slit } & 10 \mathrm{~nm} \\ \text { Room Temperature } & \end{array}$

$1 \mu \mathrm{l}$ of protein sample reaction mixture was thoroughly pipetted into $180 \mu \mathrm{l}$ of ThT working solution (glycine $50 \mathrm{mM}, \mathrm{pH} 8$ and $5 \mu \mathrm{M}$ ThT). Experiments were done in triplicates and a negative control of ThT working solution was always included. 


\subsubsection{Electron microscopy}

The morphology of the fibrils was analyzed by negative-stain transmission electron microscopy (TEM). For each sample the fibrils were harvested by ultracentrifugation discarding the supernatant followed by resuspension in fresh fibrillization buffer (50-60 $\mu \mathrm{l})$. Analysis of the samples were performed by Gudrun Heim at the facility of Transmission Electron Microscopy at the Max Planck Institute for Biophysical Chemistry, in Göttingen. Samples were transferred onto a continuous carbon coated copper grid and buffer removal was achieved using a filter paper. Negative staining was performed by adding $1 \%$ of aqu. uranyl acetate solution followed by drying process with a filter paper. The EM pictures were taken using a FEI CM 120 electron microscope with Tietz F416 CMOS camera.

\subsubsection{Circular dichroism of fibrils obtained from K32 and the P2R2 and P2R3 peptides}

Fibrils were pelleted by ultracentrifugation at $200,000 \mathrm{~g}$, at $37{ }^{\circ} \mathrm{C}$ for onehour in a TLA 100.3 rotor in a bench ultracentrifuge (Beckman Coulter, Optima MAX XP) and resuspended in $\mathrm{dd}_{2} \mathrm{O}$ to a final concentration of $20 \mu \mathrm{M}$. Measurements were performed in a $0.1 \mathrm{~cm}$ light path quartz cuvette, using general settings for the spectra acquisition as described in section 2.5 .

\subsection{In vitro tau phosphorylation with kinases}

In vitro phosphorylation of htau40 was performed by incubation of 50-100 $\mu \mathrm{M}$ of the protein with $0.4 \mu \mathrm{M}$ of Cdk2/CycA2 in a buffer containing sodium phosphate $50 \mathrm{mM}, \mathrm{pH}$ 6.8, $2 \mathrm{mM}$ DTT, $4 \mathrm{mM}$ ATP, $2 \mathrm{mM}$ EGTA, $0.5 \mathrm{mM}$ PMSF, $5 \mathrm{mM} \mathrm{MgCl}_{2}$. In vitro htau40 phosphorylation using MARK2 was performed by incubation of $50-100 \mu \mathrm{M}$ of the protein with $0.4 \mu \mathrm{M}$ of kinase in a buffer containing sodium phosphate $50 \mathrm{mM}, \mathrm{pH} 6.8,2 \mathrm{mM}$ DTT, 4 mM ATP, 2 mM EGTA, 0.5 mM PMSF, 5 mM MgCl 2.

The mixtures were incubated in a thermo block shaking at $300 \mathrm{rpm}$, for 12 hours (MARK2) and for 24 hours (Cdk2/CycA2) at $30{ }^{\circ} \mathrm{C}$ and $25{ }^{\circ} \mathrm{C}$, respectively. The kinases were inactivated by boiling the samples for 20 minutes followed by centrifugation at 13,000 
rpm for 30 minutes. The phosphorylated tau samples used for droplet formation were dialysed overnight at $4^{\circ} \mathrm{C}$ against a BRB80 buffer containing $100 \mathrm{mM}$ PIPES, pH 6.9, $1 \mathrm{mM}$ $\mathrm{MgCl}_{2,1} \mathrm{mM} \mathrm{EGTA}, 1 \mathrm{mM} \mathrm{GTP}, 1 \mathrm{mM}$ DTT. The degree of phosphorylation was performed by analysis of the cross peaks in the ${ }^{1} \mathrm{H}_{-}{ }^{15} \mathrm{~N} \mathrm{HSQC}$ spectra acquired after phosphorylation (see section 2.11.2.2).

\subsection{Phase-contrast and fluorescence microscopy}

\subsubsection{Fluorescent labeling of tau and tubulin}

HTau40 as well as pTau(Cdk2) and pTau(MARK2) proteins were fluorescently labeled using the Alexa 488 Microscale Protein Labeling Kit (Thermo Fisher Scientific, \#A30006). Porcine brain purified tubulin was fluorescently labeled using the Alexa 594 Microscale Protein Labeling Kit (Thermo Fisher Scientific, \#A30008). Subsequently, hTau40/pTau(Cdk2)/pTau(MARK2) was mixed with small amounts of Alexa 488fluorescently labeled hTau40/pTau(Cdk2) pTau(MARK2) in BRB80 buffer.

\subsubsection{Tau droplet formation}

LLPS was induced by addition of $10 \%$ dextran (Dextran T500, Cat. No. 40030, Pharmacosmos) at room temperature (final protein concentration: $25 \mu \mathrm{M}$ ). $10 \mu \mathrm{L}$ of samples were loaded onto glass slides, covered with a $18 \mathrm{~mm}$ coverslip. Differential interference contrast (DIC) and fluorescent images were acquired on a Leica DM6000B microscope with a $63 \times$ objective (water immersion) and processed using Fiji software (NIH). 


\subsection{Tubulin polymerization}

\subsubsection{Assembly of paclitaxel-stabilized microtubules}

Microtubules used for NMR spectroscopy were obtained through incubation at $37^{\circ} \mathrm{C}$ for a total of $45 \mathrm{~min}$ of $25 \mu \mathrm{M}$ tubulin in BRB80 buffer (80 mM PIPES, pH 6.9, $1 \mathrm{mM} \mathrm{MgCl}_{2} 1$ mM EGTA, $1 \mathrm{mM}$ GTP, $1 \mathrm{mM}$ DTT) to which equimolar paclitaxel (Sigma-Aldrich) was added. The suspensions of the samples were fractionated by ultracentrifugation at 40,000 $\mathrm{g}$ for $15 \mathrm{~min}$. For NMR measurements, the microtubule pellet was resuspended in $50 \mathrm{mM}$ phosphate buffer, $\mathrm{pH} 6.8$.

\subsubsection{Tubulin polymerization from tau droplets}

Tubulin polymerization assays in the presence of pre-formed tau droplets were performed in BRB80 buffer at room temperature. After formation of tau droplets, tubulin and GTP were added to a final concentration of $10 \mu \mathrm{M}$ and $1 \mathrm{mM}$, respectively.

\subsection{Synthetic peptides}

The following synthetic peptides have been used for NMR experiments described in the result sections.

$\begin{array}{lll}\text { Tau(P2) } & \text { Residues 211-242 } & \text { Ac-RTPSLPTPPTREPKKVAVVRTPPKSPSSAKSR-NH2 } \\ \text { Tau(P2)short } & \text { Residues 224-238 } & \text { Ac-KKVAVVRTPPKSPSS-NH2 } \\ \text { Tau(P2-R3) } & \text { Residues 224-313 } & \text { Ac-KKVAVVRTPPKSPSSVPGGGSVQIVYKPV-NH2 } \\ \text { Tau(R3)short } & \text { Residues 300-313 } & \text { Ac-VPGGGSVQIVYKPV-NH2 } \\ \text { 2pTau(225-246) } & \text { Residues 225-246 } & \text { Ac-KVAVVRTPPKSPSSAKSRLQTA }\end{array}$




\subsection{Nuclear magnetic resonance}

Nuclear Magnetic Resonance (NMR) spectroscopy is a non-invasive method to study protein structure and dynamics in near-native conditions ${ }^{57,148}$. NMR observes the behaviour of the nuclear spins of a molecule when these are subjected to an external magnetic field. When an external magnetic field is applied, the nuclear spin states are split into different energy levels (Figure 2.2). The size of the energy gap is directly proportional to the strength of the external magnetic field. The energy levels are not equally populated, which in a simplified way means that the probabilities for a nuclear spin to be in one state or another are different ${ }^{149}$. This difference in populations between the energy levels is the source of macroscopic magnetization and the NMR signal ${ }^{149}$. Transitions between the nuclear spin states are possible only when they take place at a frequency corresponding to the energy gap ${ }^{149,150}$. This can be observed by irradiating the spins with electromagnetic radiation having that frequency and observing the electromagnetic response of the spins. This response is a frequency defined by the nature of the nuclei and their chemical environment. The chemical shift is the position on a scale of parts per million (ppm) of this frequency and yields important structural information for the NMR spectroscopist ${ }^{150}$.

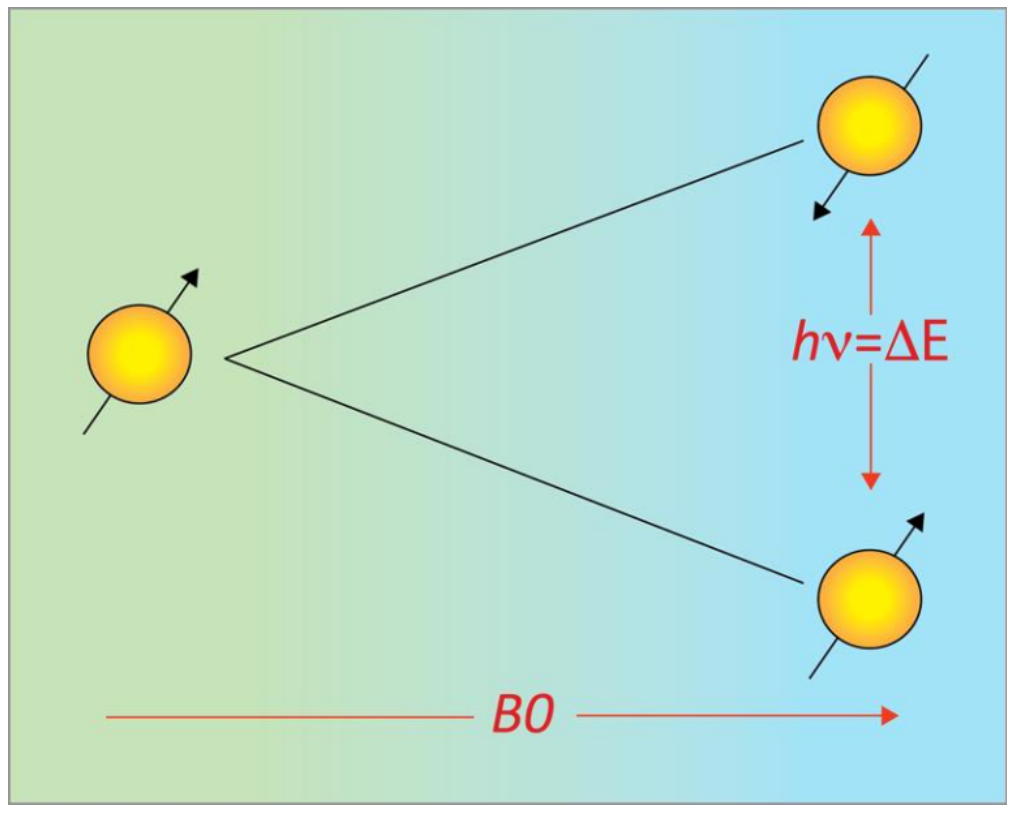

Figure 2.2. Schematic representation of the energy level splitting in an external magnetic field. When a nuclear spin is in an external magnetic field $(B O)$ its states are split into different energy levels. The energy difference $(\Delta E)$ separates one level from the other and is proportional to the size of the magnetic field. A transition between these levels is only possible when the frequency matches the energy difference between them. 
The nuclei commonly observed in protein NMR experiments are ${ }^{1} \mathrm{H},{ }^{13} \mathrm{C}$ and ${ }^{15} \mathrm{~N}$, their characteristics are summarized in Table 2.1.

Table 2.1 Gyromagnetic ratios, NMR frequencies (in a 9.4 $\mathrm{T}$ field $=\mathbf{4 0 0} \mathrm{MHz}$ ) and natural abundancies of the nuclei commonly observed in protein NMR experiments ${ }^{151}$.

\begin{tabular}{cccc} 
Isotope & $\gamma / 1 \mathbf{1 0}^{\mathbf{7}} \mathrm{T}^{-1} \mathrm{~s}^{-1}$ & $\boldsymbol{V}_{\mathrm{NMR}} / \mathrm{MHz}$ & Natural Abundance (\%) \\
\hline${ }^{1} \mathrm{H}$ & 26.752 & 400.0 & 99.985 \\
${ }^{2} \mathrm{H}$ & 4.107 & 61.4 & 0.015 \\
${ }^{13} \mathrm{C}$ & 6.728 & 100.6 & 1.108 \\
${ }^{15} \mathrm{~N}$ & -2.2713 & 40.5 & 0.37
\end{tabular}

\subsubsection{One- and two-dimensional NMR}

The simplest NMR experiment is a one-dimensional (1D) ${ }^{1} \mathrm{H}$ experiment, where the frequencies of the ${ }^{1} \mathrm{H}$ nuclei in a molecule are observed (Figure 2.3a). In protein NMR such an experiment is very complex to analyze due to the overlap of the ${ }^{1} \mathrm{H}$ signals. One way to solve this problem is to add a second dimension, which is achieved by two-dimensional (2D) NMR experiments. In a 2D NMR experiment two frequencies, $\omega_{1}$ and $\omega_{2}$, are observed. The frequencies $\omega_{1}$ and $\omega_{2}$ become the coordinates of the cross-peaks arising in the spectrum (Figure 2.3b). A similar approach is employed in three-dimensional (3D) NMR experiments, in which a third dimension is added (section 2.11.4).

$$
\text { a }
$$

a<smiles>[R]C([2H])C(=O)O</smiles>

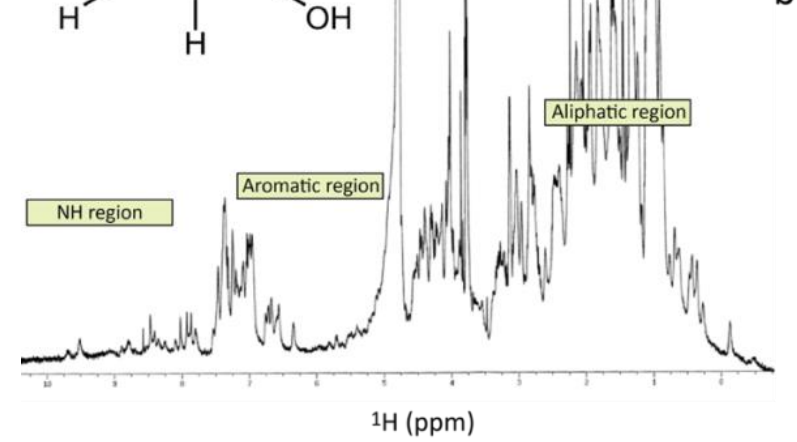

b

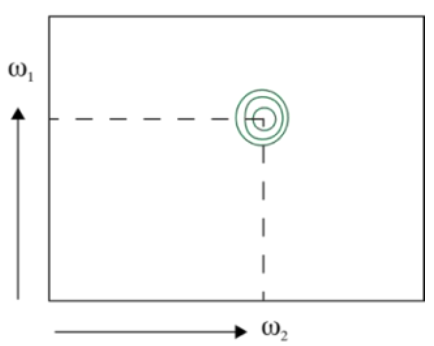

Figure 2.3. One- and two-dimensional NMR. a) A $1 D^{1} \mathrm{H}$ NMR spectrum is displayed; the regions of the spectrum where it is expected to find signals from the functional groups of the amino acid are indicated. $b$ ) Schematic representation of the basic principle of a two-dimensional NMR spectrum ${ }^{150}$. 


\subsubsection{D heteronuclear single quantum coherence (HSQC)}

2D heteronuclear single quantum coherence (HSQC) experiments are commonly used to study proteins. In a ${ }^{1} \mathrm{H}-{ }^{15} \mathrm{~N}$ HSQC, the two dimensions observed correspond to the frequencies of the ${ }^{1} \mathrm{H}$ and the ${ }^{15} \mathrm{~N}$ in the amide group of each amino acid, which are coupled across one bond. The amide group (NH) cross-peaks will appear with a specific chemical shift in the spectrum, according to their chemical environment. Thus, the ${ }^{1} \mathrm{H}-{ }^{15} \mathrm{~N}$ $\mathrm{HSQC}$ experiment provides a fingerprint of the protein. In a ${ }^{1} \mathrm{H}^{13} \mathrm{C} \mathrm{HSQC}$, information regarding all the $\mathrm{CH}$ spin-systems present in the protein, i.e. $\mathrm{C}_{\alpha} \mathrm{H}$ in the backbone and $\mathrm{CH}$ moieties in the side chains, are obtained ${ }^{152}$. As shown in Table $1.2,{ }^{1} \mathrm{H},{ }^{15} \mathrm{~N}$ and ${ }^{13} \mathrm{C}$ do not have the same natural abundance: for this reason, in order to perform ${ }^{1} \mathrm{H}_{-}{ }^{15} \mathrm{~N}$ or ${ }^{1} \mathrm{H}_{-}{ }^{13} \mathrm{C} 2 \mathrm{D}$ experiments, recombinant proteins are enriched with ${ }^{15} \mathrm{~N}$ and ${ }^{13} \mathrm{C}$ isotopes, a strategy known as isotopic labeling. Figure 2.4 shows the pulse sequence of a standard ${ }^{1} \mathrm{H}-{ }^{15} \mathrm{~N} \mathrm{HSQC}$ experiment. A pulse sequence consists of a series of short irradiations designed to encode the frequency information of the observed nuclei in the NMR signal. The pulse sequence of a 2D experiment consists of four periods, preparation, evolution $\left(t_{1}\right)$, mixing and detection $\left(t_{2}\right)$. During the preparation period of an HSQC experiment, transfer of magnetization from the ${ }^{1} \mathrm{H}$ to ${ }^{15} \mathrm{~N}$ is achieved by a sequence of pulses, called Insensitive Nuclei Enhancement by Polarization Transfer (INEPT) ${ }^{153}$.

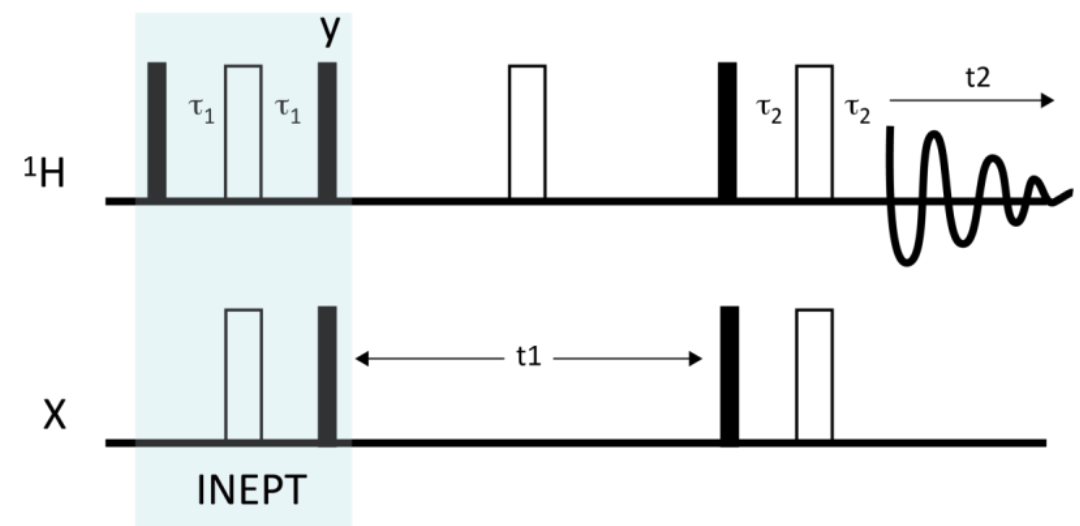

Figure 2.4. Simplified 2D HSQC pulse sequence.The HSQC pulse sequence starts with equilibrium magnetization on ${ }^{1} \mathrm{H}$. Magnetization is transferred to the insensitive nucleus $X\left({ }^{15} \mathrm{~N}\right.$ or ${ }^{13} \mathrm{C}$ ) via INEPT (lightblue box): $90^{\circ} \mathrm{x}-180^{\circ} \mathrm{y}-90^{\circ} \mathrm{y}$ rf pulses are applied on ${ }^{1} \mathrm{H}$; the delay $\tau$ is set to $\sim 2.7 \mathrm{~ms}$ for ${ }^{1} \mathrm{H}-{ }^{15} \mathrm{~N} \mathrm{HSQC}$ and to $1.7 \mathrm{~ms}$ for ${ }^{1} \mathrm{H}-{ }^{13} \mathrm{C}$ HSQC. The chemical shift of the coupled spin evolves during $t_{1}$. Using a reverse-INEPT sequence, magnetization is transferred back to ${ }^{1} \mathrm{H}$, where the signal is detected. 
With this sequence, magnetization is transferred from a sensitive (i.e. ${ }^{1} \mathrm{H}$ ) to a comparatively insensitive nuclei (i.e. ${ }^{13} \mathrm{C}$ or ${ }^{15} \mathrm{~N}$ ) using scalar $J$-coupling. The result is an enhancement of the intensity of the insensitive nuclei. After magnetization has been transferred to the ${ }^{15} \mathrm{~N}$, its chemical shift evolves during the evolution period. In the mixing period, the magnetization is transferred back to the ${ }^{1} \mathrm{H}$, on which the signal is detected. The pulse sequence of an HSQC can also be combined with other NMR experiments to obtain more complex and sophisticated experiments.

\subsubsection{HSQC experiment settings and data analysis for the project I}

In the first project, 2D HSQC experiments have been used to study the K32 construct. 2D ${ }^{1} \mathrm{H}_{-}{ }^{13} \mathrm{C}$ and ${ }^{1} \mathrm{H}_{-}{ }^{15} \mathrm{~N}$ HSQC of $200 \mu \mathrm{M}$ uniformly ${ }^{13} \mathrm{C}_{-}{ }^{15} \mathrm{~N}$ labeled $\mathrm{K} 32$ in $50 \mathrm{mM}$ sodium phosphate buffer, $\mathrm{pH} 6.8,10 \% \mathrm{D}_{2} \mathrm{O}, 0.02 \%$ sodium azide and $2 \mathrm{mM} \mathrm{DTT}$, added freshly before the measurement, were recorded at $5{ }^{\circ} \mathrm{C}$. Spectra were acquired on a $700 \mathrm{MHz}$ spectrometer equipped with a cryogenically-cooled triple resonance probe (Bruker).

In order to characterize the P2R2 and P2R3 tau peptides, $2 \mathrm{D}{ }^{1} \mathrm{H}-{ }^{13} \mathrm{C}$ and ${ }^{1} \mathrm{H}-{ }^{15} \mathrm{~N}$ HSQCs were recorded at $5{ }^{\circ} \mathrm{C}$ on $1 \mathrm{mM}$ concentrated samples of uniformly ${ }^{13} \mathrm{C} /{ }^{15} \mathrm{~N}$ labeled peptides in $50 \mathrm{mM}$ sodium phosphate buffer, $\mathrm{pH} 6.8,10 \% \mathrm{D}_{2} \mathrm{O}$ and $0.02 \%$ sodium azide. Spectra of P2R2 were acquired on Bruker Avance III HD spectrometers operating at $600 \mathrm{MHz}$ (Bruker) and spectra of P2R3 were acquired on a $600 \mathrm{MHz}$ Bruker spectrometer equipped with a triple-resonance cryogenically-cooled NMR probe. The pulse program used for the acquisition of ${ }^{1} \mathrm{H}-{ }^{15} \mathrm{~N} \mathrm{HSQC}$ is a user modified pulse sequence (see Appendix: hsqc15N.dl) while for the ${ }^{1} \mathrm{H}^{13} \mathrm{C}$ HSQC a standard Bruker (Appendix: hsqcetgpsi) pulse program was used. Experimental parameters are summarized in Table 2.2. All spectra were processed using Topspin 3.5pl7 (Bruker) and further analysed using the Sparky ${ }^{154}$ and ccpNMR software ${ }^{155}$.

\subsubsection{HSQC experiment settings and data analysis for the project II}

In the second project, 2D ${ }^{1} \mathrm{H}_{-}{ }^{15} \mathrm{~N}$ HSQC experiments were used to characterize the phosphorylated state of pTau(Cdk2/CycA2)/pTauMARK2 and to study the interaction of htau40/pTau(Cdk2/CycA2)/pTauMARK2 with tubulin/microtubules. 
To study the temperature dependence of the interaction between htau40 and microtubules, 2D ${ }^{1} \mathrm{H}-{ }^{15} \mathrm{~N} \mathrm{HSQ}$ spectra were acquired at $5{ }^{\circ} \mathrm{C}, 25{ }^{\circ} \mathrm{C}$ and $37{ }^{\circ} \mathrm{C}$ on $50 \mu \mathrm{M}$ htau40 alone or in presence of $25 \mu \mathrm{M}$ of paclitaxel-stabilized microtubules. The NMR samples were prepared in $50 \mathrm{mM}$ sodium phosphate buffer $50 \mathrm{mM}, \mathrm{pH} 6.8,10 \% \mathrm{D}_{2} \mathrm{O}$ and $0.02 \%$ sodium azide. Experiments were performed on a $700 \mathrm{MHz}$ spectrometer (Bruker) equipped with a cryogenically-cooled triple resonance probe.

For the interaction between htau40/pTau(Cdk2/CycA2) and soluble tubulin, $2 \mathrm{D}^{1} \mathrm{H}-$ ${ }^{15} \mathrm{~N} \mathrm{HSQ}$ spectra were acquired at $5{ }^{\circ} \mathrm{Con} 10 \mu \mathrm{M}$ htau40/pTau(Cdk2/CycA2) alone or in presence of $5 \mu \mathrm{M}$ of paclitaxel-stabilized microtubules. The NMR samples were prepared in $50 \mathrm{mM}$ sodium phosphate buffer $50 \mathrm{mM}, \mathrm{pH} 6.8,10 \% \mathrm{D}_{2} \mathrm{O}$ and $0.02 \%$ sodium azide. Experiments were recorded at $5^{\circ} \mathrm{C}$ on a Bruker $900 \mathrm{MHz}$ spectrometer equipped with a cryoprobe.

For characterization of the phosphorylation state of htau40/pTau(Cdk2/CycA2)/pTauMARK2, HSQC spectra of $10 \mu \mathrm{M}$ protein were recorded in $50 \mathrm{mM}$ sodium phosphate buffer, $\mathrm{pH} 6.8,10 \% \mathrm{D}_{2} \mathrm{O}$ and $0.02 \%$ sodium azide. Experiments were recorded at $5{ }^{\circ} \mathrm{C}$ on a Bruker $800 \mathrm{MHz}$ spectrometer equipped with a cryoprobe. The quantification of the unphosphorylated and phosphorylated forms of each selected residue was performed by analysis of the cross peaks in the ${ }^{1} \mathrm{H}-{ }^{15} \mathrm{~N} \mathrm{HSQC}$ spectra. The intensity of the cross peaks in the unphosphorylated ( $I_{\text {unphosphorylated }}$ ) and phosphorylated ( $\left.I_{\text {phosphorylated }}\right)$ form were summed to give a total intensity $\left(I_{\text {TOT }}\right)$. The calculation of the degree of phosphorylation was then performed using:

$$
\begin{gathered}
\text { Degree of unphosphorylated }=\frac{I_{\text {unphosphorylated }}}{I_{T O T}} \\
\text { Degree of phosphorylated }=\frac{I_{\text {phosphorylated }}}{I_{T O T}}
\end{gathered}
$$

The error propagation was calculated using the estimated mean error of each spectrum as provided by the Sparky software ${ }^{154}$.

The NMR measurements to detect the guanidium protons of arginine side chains were performed on $80 \mu \mathrm{M}$ htau40/pTau(Cdk2/CycA2) at $5{ }^{\circ} \mathrm{C}$ on a Bruker $900 \mathrm{MHz}$ 
spectrometer. In order to decrease the exchange of the labile protons with bulk solvent, a sodium phosphate buffer at $\mathrm{pH} 6$ was used. The pulse program used for the acquisition of ${ }^{1} \mathrm{H}-{ }^{15} \mathrm{~N}$ HSQC is a user modified pulse sequence (see Appendix: hsqc15N.dl). Experimental parameters are summarized in Table 2.3. Spectra were processed using Topspin 3.5pl7 (Bruker) and NMRpipe ${ }^{156}$. Further analysis wasperformed using the Sparky software ${ }^{154}$. Identification of the phosphorylated residues was achieved by chemical shift perturbation analysis. ${ }^{1} \mathrm{H}$ and ${ }^{15} \mathrm{~N}$ normalized weighted average chemical shifts were calculated using:

$$
\Delta \delta N H=\sqrt{\left[\left(\Delta H^{2}\right)+(\Delta N / 5)^{2}\right] / 2}
$$

where $\Delta H$ and $\Delta N$ correspond to the ${ }^{1} \mathrm{H}$ and ${ }^{15} \mathrm{~N}$ chemical shift differences between hTau40 and pTau(Cdk2)/pTau(MARK2). Resonance assignments of htau40 and phosphorylated residues were reported previously ${ }^{48,49,56}$.

Table 2.2. Parameters for ${ }^{1} \mathrm{H}-{ }^{15} \mathrm{~N}$ HSQC and ${ }^{1} \mathrm{H}-{ }^{13} \mathrm{C}$ HSQC experiments related to the project I.

\begin{tabular}{|c|c|c|c|c|c|c|}
\hline \multirow{2}{*}{ Protein } & \multirow{2}{*}{$\begin{array}{c}\text { Type of } \\
\text { experiment }\end{array}$} & \multirow{2}{*}{$\begin{array}{c}\text { Spectrometer } \\
\text { (MHz) }\end{array}$} & \multicolumn{2}{|c|}{ SW (ppm) } & \multirow{2}{*}{ NS } & \multirow{2}{*}{ TD } \\
\hline & & & ${ }^{1} \mathrm{H}$ & ${ }^{15} \mathrm{~N} /{ }^{13} \mathrm{C}$ & & \\
\hline \multirow[t]{2}{*}{$\mathrm{P} 2 \mathrm{R} 2$} & ${ }^{1} \mathrm{H}-{ }^{15} \mathrm{~N}$ HSQC & 600 & 12.02 & 23.00 & 8 & $2048\left({ }^{1} \mathrm{H}\right), 128\left({ }^{15} \mathrm{~N}\right)$ \\
\hline & ${ }^{1} \mathrm{H}-{ }^{13} \mathrm{C}$ HSQC & 600 & 13.02 & 75.00 & 8 & $1024\left({ }^{1} \mathrm{H}\right), 256\left({ }^{13} \mathrm{C}\right)$ \\
\hline \multirow[t]{2}{*}{ P2R3 } & ${ }^{1} \mathrm{H}-{ }^{15} \mathrm{~N}$ HSQC & 600 & 12.02 & 23.00 & 8 & $2048\left({ }^{1} \mathrm{H}\right), 128\left({ }^{15} \mathrm{~N}\right)$ \\
\hline & ${ }^{1} \mathrm{H}-{ }^{13} \mathrm{C}$ HSQC & 600 & 13.02 & 75.00 & 8 & $1024\left({ }^{1} \mathrm{H}\right), 256\left({ }^{13} \mathrm{C}\right)$ \\
\hline \multirow[t]{2}{*}{ K32 } & ${ }^{1} \mathrm{H}-{ }^{15} \mathrm{~N}$ HSQC & 700 & 12.02 & 28.00 & 64 & $2048\left({ }^{1} \mathrm{H}\right), 512\left({ }^{15} \mathrm{~N}\right)$ \\
\hline & ${ }^{1} \mathrm{H}-{ }^{13} \mathrm{C}$ HSQC & 700 & 14.00 & 149.47 & 64 & $1024\left({ }^{1} \mathrm{H}\right), 512\left({ }^{13} \mathrm{C}\right)$ \\
\hline
\end{tabular}


Table 2.3 Selected parameters for ${ }^{1} \mathrm{H}-{ }^{15} \mathrm{~N}$ HSQC experiments related to the project II

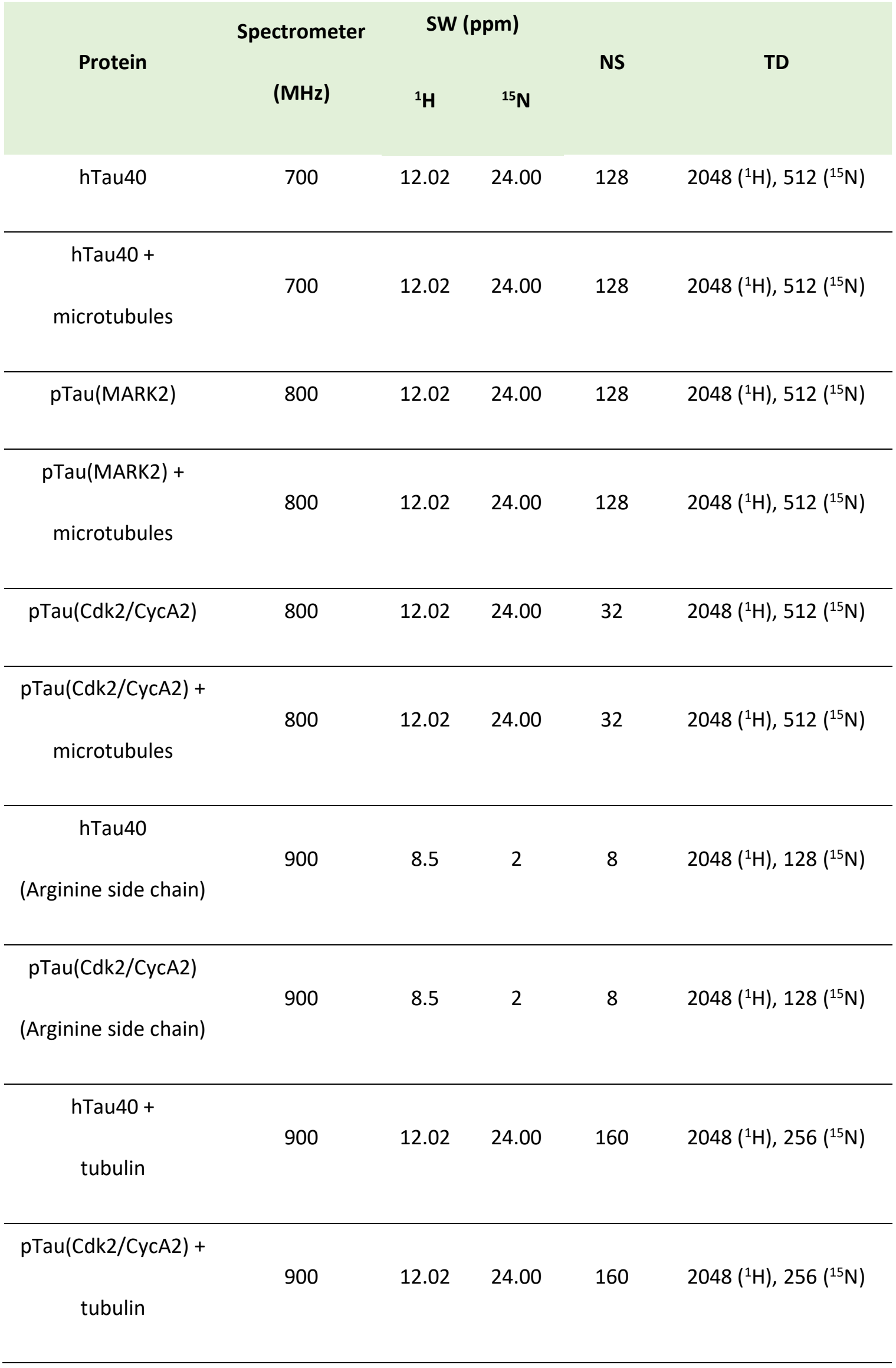




\subsubsection{Transverse relaxation-optimized spectroscopy (TROSY)}

For large molecules, the NMR signal is affected by the short transverse relaxation rate $\left(T_{2}\right)$, which causes a fast signal decay and broad peaks ${ }^{150}$. Transverse relaxation-optimized spectroscopy (TROSY) ${ }^{157}$ is used for the study of large proteins, where due to the increased molecular weight resolution would be poor and peaks would be broad in a HSQC spectrum $^{158}$. The TROSY experiment gives the same information as the HSQC but mitigates this problem by selecting those signal components, which are relaxing more slowly and cancelling those that are relaxing more quickly. Generally, the selection of these signal components halves their intensity to $1 / 2$ in each dimension. Nevertheless, the application of TROSY experiments on large proteins has the net effect of improving resolution and sensitivity ${ }^{158}$. Its principle can be extended to 3D experiments, in order to facilitate the assignment of large proteins ${ }^{159}$.

\subsubsection{TROSY experiment settings and data analysis}

2D ${ }^{1} \mathrm{H}^{13} \mathrm{C}$ and ${ }^{1} \mathrm{H}-{ }^{15}$ TROSY experiments were performed on ${ }^{15} \mathrm{~N}$-labeled full-length tau (441 residues) in presence and absence of paclitaxel-stabilized microtubules on a $700 \mathrm{MHz}$ spectrometer equipped with a cryogenically-cooled triple resonance probe (Bruker). Samples of $50 \mu \mathrm{M}$ htau40 in sodium phosphate buffer $50 \mathrm{mM}, \mathrm{pH} 6.8,0.02 \%$ sodium azide were measured at $5{ }^{\circ} \mathrm{C}, 20^{\circ} \mathrm{C}$ and $37^{\circ} \mathrm{C}$ to observe differences in the interaction depending on temperature. For the experiment, a standard Bruker sequence (see Appendix: trosyf3gpphsi19) was used; for each experiment 512 points were acquired in the ${ }^{15} \mathrm{~N}$ dimension for a total of 32 scans. Spectra were processed using Topspin $3.5 \mathrm{pl} 7$ (Bruker) and were further analysed using the Sparky software ${ }^{154}$.

\subsubsection{D NMR experiments for resonance assignment}

Triple-resonance experiments enable the assignment of uniformly ${ }^{13} \mathrm{C} /{ }^{15} \mathrm{~N}$-labeled proteins and overcome the problem of signal overlap, observed in $2 \mathrm{D}$ experiments for large proteins ( $\mathrm{MW}>30 \mathrm{kDa}$ ). In this case, the position of the cross-peaks in the spectrum are defined by three different coordinates with offsets $\Omega_{\mathrm{H}} \Omega_{\mathrm{C}}$ and $\Omega_{\mathrm{N}}$. 


\subsubsection{3D experiments settings and data analysis}

For the sequential backbone assignment of P2R2 and P2R3, a suite of triple-resonance experiments (HNCA, HNCACB, HNCACO, HNCO) was recorded on a $600 \mathrm{MHz}$ Bruker Avance III HD spectrometer equipped with a room temperature triple resonance probe as well as on a $600 \mathrm{MHz}$ Bruker spectrometer equipped with a triple-resonance cryogenically-cooled NMR probe. Experiments for sidechain assignment $((\mathrm{H}) \mathrm{CC}(\mathrm{CO}) \mathrm{NH}-$ and $\mathrm{H}(\mathrm{CC})(\mathrm{CO}) \mathrm{NH}-\mathrm{TOCSY}$ ) were acquired on a $700 \mathrm{MHz}$ spectrometer equipped with a cryogenically-cooled triple resonance probe (Bruker).

For the K32 construct, HNCA, HNCACB and HNCO spectra for backbone assignment and $((\mathrm{H}) \mathrm{CC}(\mathrm{CO}) \mathrm{NH}$ - and $\mathrm{H}(\mathrm{CC})(\mathrm{CO}) \mathrm{NH}-\mathrm{TOCSY})$ spectra for side chain assignment were recorded on a $700 \mathrm{MHz}$ spectrometer equipped with a cryogenically-cooled triple resonance probe (Bruker).

Pulse programs from the Bruker catalogue were used for the experiments described in this section (Table 2.4). Spectra were processed using Topspin $3.5 \mathrm{pl} 7$ software and assignments was performed with ccpNMR software ${ }^{155}$. In addition, a fulllength human tau assignment (BMRB entry doi:10.13018/BMR17920) was used to validate the assignment of each construct. 
Table 2.4. List of 3D experiments recorded on K32, P2R2 and P2R3.

\begin{tabular}{|c|c|c|c|c|}
\hline Protein & $\begin{array}{c}\text { Type of } \\
\text { experiment }\end{array}$ & $\begin{array}{l}\text { Spectrometer } \\
\text { (MHz) }\end{array}$ & $\begin{array}{c}\text { Pulse } \\
\text { sequence }\end{array}$ & TD \\
\hline \multicolumn{5}{|r|}{$2048\left({ }^{1} \mathrm{H}\right), 48\left({ }^{13} \mathrm{C}\right), 128\left({ }^{15} \mathrm{~N}\right)$} \\
\hline & $\begin{array}{l}\text { HNCO } \\
\text { HNCACB }\end{array}$ & 600 & $\begin{array}{l}\text { hncogp3d } \\
\text { hncacbgp3d }\end{array}$ & $\begin{array}{l}2048\left({ }^{1} \mathrm{H}\right), 48\left({ }^{13} \mathrm{C}\right), 128\left({ }^{15} \mathrm{~N}\right) \\
2048\left({ }^{1} \mathrm{H}\right), 48\left({ }^{13} \mathrm{C}\right), 128\left({ }^{15} \mathrm{~N}\right)\end{array}$ \\
\hline & $\begin{array}{l}\mathrm{HN}(\mathrm{CO}) \mathrm{CACB} \\
\mathrm{H}(\mathrm{CC})(\mathrm{CO}) \mathrm{NH}- \\
\text { TOCSY }\end{array}$ & 600 & hccconhgp3d2 & $2048\left({ }^{1} \mathrm{H}\right), 64\left({ }^{13} \mathrm{C}\right), 176\left({ }^{15} \mathrm{~N}\right)$ \\
\hline & $\begin{array}{c}(\mathrm{H}) \mathrm{CC}(\mathrm{CO}) \mathrm{NH}- \\
\text { TOCSY }\end{array}$ & 700 & hccconhgp3d3 & $2048\left({ }^{1} \mathrm{H}\right), 40\left({ }^{13} \mathrm{C}\right), 128\left({ }^{15} \mathrm{~N}\right)$ \\
\hline \multirow[t]{5}{*}{ K32 } & HNCA & 700 & hncagp3d & $2048\left({ }^{1} \mathrm{H}\right), 64\left({ }^{13} \mathrm{C}\right), 128\left({ }^{15} \mathrm{~N}\right)$ \\
\hline & HNCO & 700 & hncogp3d & $2048\left({ }^{1} \mathrm{H}\right), 64\left({ }^{13} \mathrm{C}^{\prime}\right), 200\left({ }^{15} \mathrm{~N}\right)$ \\
\hline & HNCACB & 700 & hncacbgp3d & $2048\left({ }^{1} H\right), 64\left({ }^{13} C^{\prime}\right), 200\left({ }^{15} N\right)$ \\
\hline & $\begin{array}{c}\mathrm{H}(\mathrm{CC})(\mathrm{CO}) \mathrm{NH}- \\
\text { TOCSY }\end{array}$ & 700 & hccconhgp3d2 & $2048\left({ }^{1} \mathrm{H}\right), 64\left({ }^{13} \mathrm{C}^{\prime}\right), 200\left({ }^{15} \mathrm{~N}\right)$ \\
\hline & $\begin{array}{c}(\mathrm{H}) \mathrm{CC}(\mathrm{CO}) \mathrm{NH}- \\
\text { TOCSY }\end{array}$ & 700 & hccconhgp3d3 & $2048\left({ }^{1} \mathrm{H}\right), 80\left({ }^{13} \mathrm{C}^{\prime}\right), 136\left({ }^{15} \mathrm{~N}\right)$ \\
\hline
\end{tabular}

Abbreviations: TD, time domain. 


\subsubsection{The Nuclear Overhauser Effect}

The Nuclear Overhauser Effect (NOE) causes the through space transfer of magnetization between two or more spins. In the NMR spectrum, it causes a change in the intensity of a peak when another nearby peak (distance $<5-6 \AA$ ) is saturated ${ }^{150}$. Saturation is the process by which the irradiation of a spin equalizes its population difference and leads to the disappearance of the peak in the NMR spectrum ${ }^{160}$.

The NOE is directly correlated to the distance between the two spins, which makes it a powerful technique to obtain structural information ${ }^{160}$. By measuring nuclear Overhauser effect spectroscopy (NOESY) experiments intra- and intermolecular restraints are obtained.

\subsubsection{The transferred NOE (Tr-NOE)}

NOESY experiments can also be performed to understand the conformation acquired by a target protein/peptide binding to a partner as in the case of transferred-NOE (Tr-NOEs) experiments ${ }^{160}$. It is a specific case of NOE in which the information of the bound state is detected on the ligand in its free form. It is based on the assumptions, that when chemical exchange between two interacting proteins occurs on the fast-exchange limit of the NMR time scale, the structural information of the bound-state remains encoded once the ligand returns to its free state ${ }^{161}$.

\subsubsection{2D NOE experiments for the structure determination of Tau(P2)}

All the NOESY experiments described in this section, as well as the structure calculation that derived from them were performed by Dr. Harindranath Kadavath. Tr-NOESY spectra ${ }^{162}$ were recordedat $5{ }^{\circ} \mathrm{C}$ on a Bruker $900 \mathrm{MHz}$ spectrometer equipped with a cryoprobe. Samples contained $1.0 \mathrm{mM}$ peptide and $50 \mu \mathrm{M}$ of paclitaxel-stabilized microtubules. The tau-to-microtubule molar ratio was 20:1. The buffer contained $50 \mathrm{mM}$ sodium phosphate, $\mathrm{pH} 6.8,10 \% \mathrm{D}_{2} \mathrm{O}$. NOE mixing times used for the experiments were 50 and $100 \mathrm{~ms}$. For the structure calculation, distance restraints were obtained from tr- 
NOE contacts observed in $2 \mathrm{D}{ }^{1} \mathrm{H}-{ }^{1} \mathrm{H}$ NOESY spectra acquired with $100 \mathrm{~ms}$ mixing time. Initial structure calculations were performed using CYANA 3.0 and 200 conformers were calculated using the standard simulated annealing schedule with 10,000 torsion angle dynamics steps per conformer. Subsequently, the structures derived from CYANA were refined in XPLOR-NIH using a restrained simulated annealing protocol. ${ }^{163}$ Default values were used for force constants and molecular parameters unless otherwise indicated. 200 conformers were calculated and the 20 lowest-energy conformers were selected for further analysis using iCing (https://nmr.le.ac.uk/icing/\#file). Visualization was performed using MOLMOL ${ }^{164}$ and PYMOL (The PyMOL Molecular Graphics System, Version 1.5.0.4 Schrödinger, LLC).

\subsubsection{Saturation transfer difference (STD)}

Saturation transfer difference (STD) NMR spectroscopy is a robust ligand-based screening method for studying protein-ligand interactions at an atomic level ${ }^{165-167}$. This method can be applied to a wide range of molecules and requires low amount of sample, therefore it is a very useful tool for NMR-based screening ${ }^{168}$.

The experiment is based on the acquisition of two spectra, called on-resonance and off-resonance spectra. During acquisition of the on-resonance spectrum, the protein is irradiated for a defined saturation time $\left(T_{\text {sat }}\right)$ at a specific frequency, often between 0.0 and $-1.0 \mathrm{ppm}$. This is done to ensure that only resonances belonging to the protein, and not to the ligand, are saturated. During the acquisition of the off-resonance spectrum the offset of the irradiation is set far from any protein resonance, for instance 30-60 ppm, therefore no signals are perturbed and the spectrum is used as reference for further analysis. When the protein is irradiated, magnetization is quickly transferred to all its protons via spin diffusion, due to the large size of the protein (>30 $\mathrm{kDa})$. When the ligand binds, saturation is transferred to it via intermolecular NOE, causing an intensity attenuation of protons in close contact with the protein surface. 


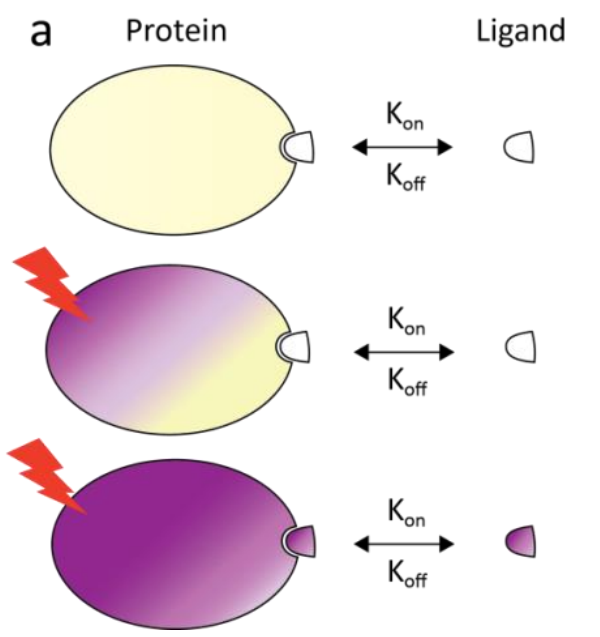

b
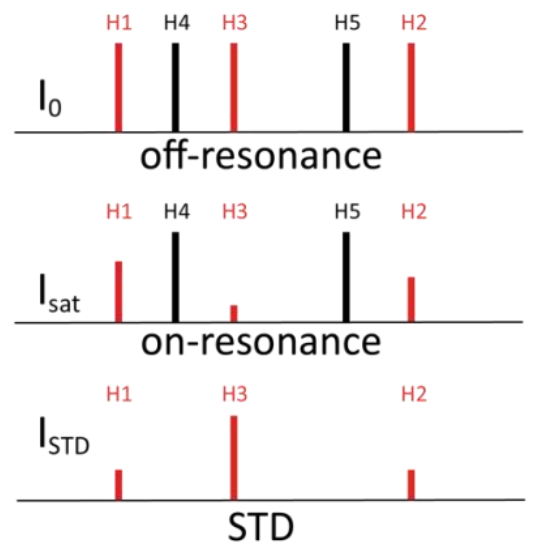

Figure 2.5. Schematic representation of the STD NMR experiment.a) The protein is selectively irradiated for a fixed interval of time $\left(T_{\text {sat }}\right)$ and magnetization is transferred via spin diffusion among its protons. When the ligand binds the protein, protons at close distance $(<5-6 \AA)$ are saturated. Because of the fast-exchange regime, the ligand returns to the free-state before magnetization is lost and saturation is conserved due to its small relaxation rate, giving rise to the STD effect (Adapted from Yu et al., 2015). b) An off-resonance experiment is acquired, setting the irradiation frequency far from protein resonances, the signal intensity $\left(I_{0}\right)$ is not perturbed; an on-resonance experiment instead selectively irradiates the protein $\left(I_{\text {sat }}\right)$. The STD spectrum is obtained from the difference of the off- and on-resonance spectra. Adapted from Krishnan, Current Analytical Chemistry, 2005.

In the fast-exchange NMR limit, the ligand dissociates from the protein before it loses magnetization and its saturated state persists also when the ligand is free in solution ${ }^{165}$ (Figure 2.5a). The STD spectrum, is the difference of the off- and on-resonance spectra and the STD intensity for each proton, $I_{S T D}$, is the difference of its signal intensity in the off-resonance and the on-resonance spectra, $I_{0}-I_{\text {sat }}$. STD signals thus belong to the ligand protons, which were saturated in the on-resonance spectrum ${ }^{165,166,169}$ (Figure 2.5b).

\subsubsection{STD NMR experiments settings and data analysis}

All STD NMR samples were prepared in BRB80 buffer (80 mM PIPES, pH 6.8, $1 \mathrm{mM} \mathrm{MgSO}_{4}$, $1 \mathrm{mM}$ EGTA, $1 \mathrm{mM}$ DTT) supplemented with $50 \mathrm{mM} \mathrm{KCl}$ and $5 \mathrm{mM} \mathrm{CaCl}_{2}$ to achieve nonpolymerizing condition. Porcine brain tubulin samples used for the experiments were purchased from Cytoskeleton, Inc. In addition, porcine tubulin was kindly provided by the laboratory of Prof. Dr. Eckhard Mandelkow. Tubulin concentration was kept fixed at $5 \mu \mathrm{M}$, while tau(P2) concentrations varied from $50 \mu \mathrm{M}$ to $1.5 \mathrm{mM}$. The spectra were acquired on 
a $700 \mathrm{MHz}$ spectrometer equipped with a cryogenically-cooled triple resonance probe (Bruker). A standard pseudo-2D pulse sequence (appendix: stdiffgp19.2) was used. Figure 2.6 represents the stdiffgp19.2 Bruker sequence used for the STD experiment: selective irradiation of tubulin is achieved using a train of 50 ms gaussian-shaped pulse, at a power level of $46 \mathrm{~dB}$ and a 3-9-19 WATERGATE is used for solvent suppression before the detection. The on-resonance frequency was set to $-0.5 \mathrm{ppm}$ and the off-resonance to 60 ppm. One advantage of using this pulse sequence is that the on- and off-resonance spectra are acquired in an alternate fashion for the duration of the entire experiment giving an internal reference.

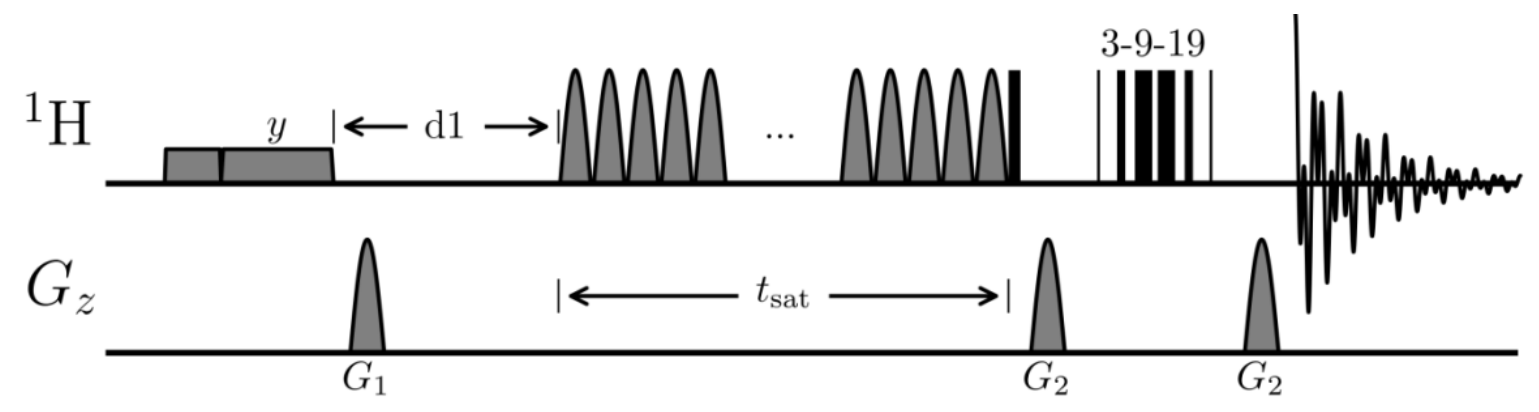

Figure 2.6. STD standard Bruker pulse sequence.The stddiffgp19.2 avance-version of the standard STD pulse program is a pseudo-2D sequence where the two channels are both targeting proton resonances. A train of selective gaussian-shaped $90^{\circ}$ pulses of the duration of $50 \mathrm{~ms}$ at $46 \mathrm{~dB}$ is saturating the protein: duration and power level of this pulse are adjusted to obtain selectivity and efficient saturation at the same time. Resonance frequencies are defined in the FQ2LIST of the acquisition parameters for the on- and offresonance irradiation. A $90^{\circ}$ pulse brings magnetization to the transverse plane and is followed by a 3-9-19 sequence to suppress the water signal.

For the determination of binding profiles, $1 \mathrm{D}^{1} \mathrm{H}$ STD experiments were acquired at $5{ }^{\circ} \mathrm{C}$ using $5 \mu \mathrm{M}$ of tubulin and $800 \mu \mathrm{M}$ of either $\mathrm{K} 32$ or peptides. Increasing irradiation times $(0.5,1,1.5,2,3,4,5$ and $7 \mathrm{sec})$ were used to obtained a signal buildup curve. Spectra were processed and analyzed using Topspin $3.5 \mathrm{pl} 7$ (Bruker): sharp and isolated peaks from the amide or methyl region of $1 \mathrm{D}{ }^{1} \mathrm{H}$ spectra were selected for analysis. For each selected region, the absolute integral values from the on- and off-resonance spectra were used for the calculation of the STD effect $\left(\eta_{S T D}\right)$ as shown in eq (5).

$$
\eta_{S T D}=\frac{I_{0}-I_{S T D}}{I_{0}}
$$


The intensity of each selected peak for different irradiation times, $A_{\operatorname{STD}\left(t_{s a t}\right)}$, was calculated by multiplying its $\eta_{S T D}$ value for the ligand excess. For each peak, the obtained $A_{S T D\left(t_{s a t}\right)}$ was plotted as a function of the irradiation time. The $S T D_{\max }$ was the STD amplification factor at the plateau of the curve. The experimental values were fitted using eq (6), from which $k_{\text {sat }}$ were obtained.

$$
A_{S T D_{\left(t_{s a t}\right)}}=S T D_{\max } \cdot\left(1-\exp \left(-k_{\text {sat }} \cdot t_{\text {sat }}\right)\right)
$$

For the quantification of the $K_{D}$ values of Tau(P2), $1 \mathrm{D}^{1} \mathrm{H}$ STD spectra were acquired at $5{ }^{\circ} \mathrm{C}$ using $5 \mu \mathrm{M}$ of tubulin and50 $\mu \mathrm{M}, 100 \mu \mathrm{M}, 200 \mu \mathrm{M}, 300 \mu \mathrm{M}, 400 \mu \mathrm{M}, 800 \mu \mathrm{M}, 1 \mathrm{mM}$ and $1.5 \mathrm{mM}$ of peptide. An irradiation time of $2 \mathrm{~s}$ was selected as optimal interval. Spectra were processed and analyzed using Topspin $3.5 \mathrm{pl}$ (Bruker): the same sharp and isolated peaks which were selected for the binding profiles were analysed. The STD effect, $\eta_{S T D}$, was calculated using eq (5) in a similar way as mentioned before: the $A_{S T D}$ was calculated as the product of $\eta_{S T D}$ and the ligand excess. $A_{S T D}$ obtained for each selected peak were plotted as a function of the ligand concentration. The $K_{D}$ was determined by fitting the obtained values to eq (7):

$$
A_{S T D}=\frac{\left(\alpha_{S T D} \cdot[L]\right)}{\left(K_{D}+[L]\right)}
$$

where for $[L]$ each ligand concentration used was considered. Error bars where calculated using:

$$
\text { error }=A F \cdot \sqrt{\left(\frac{N}{S}\right)_{I_{S T D}}^{2}+\left(\frac{N}{S}\right)_{I_{0}}^{2}}
$$

Here, $\frac{N}{S}$ was calculated as the reciprocal of the signal-to-noise ratio, $\frac{S}{N}$, of each peak selected in the on- and off- resonance spectra: the noise region was the integral of a region spacing from 10 to $9.5 \mathrm{ppm}$, where no protein signal was detected, while the signal region was the integral of each peak for which the analysis was performed. The standard error for $K_{D}$ values was obtained using Graph Prism version 8.0 (GraphPad Software, La Jolla California USA, www.graphpad.com). 


\subsubsection{Characterization of protein diffusion via NMR}

Diffusion NMR experiments are often used to investigate the diffusional properties of molecules in solution. The quantification of the self-diffusion coefficient provides information about the size of a given molecule and can be related to its structural organization ${ }^{170,171}$. The self-diffusion coefficient is obtained by the measurement of the diffusion rate of a given molecule in the presence of a gradient magnetic field and can be related to the molecule hydrodynamic radius $\left(r_{H}\right)$. The effect of the gradient magnetic field is different for molecules diffusing at faster or slower rates and is observed in NMR in terms of signal attenuation ${ }^{172}$.

\subsubsection{NMR diffusion experiment settings and data analysis}

The self-diffusion coefficients of P2R2 and P2R3 were obtained by performing pulse gradient stimulated echo (PGSTE) WATERGATE experiments ${ }^{173}$ on a 600 Avance III HD (Bruker). A user-modified pulse sequence (see Appendix: PGSTE_WATERGATE_2D.nare) was used to perform the experiments. Experiments were performed at $5{ }^{\circ} \mathrm{C}$ on samples of either P2R2 or P2R3 at $1 \mathrm{mM}$ concentration, in a sodium phosphate buffer $50 \mathrm{mM}, \mathrm{pH}$ 6.8. Additional $1 \mathrm{mM}$ of DSS (4,4-dimethyl-4-silapentane-1-sulfonic acid) was added to each NMR sample as standard for the calculation of the hydrodynamic radii. Parameters $\Delta$ and $\delta$ were optimized according to the samples to $500 \mathrm{~ms}$ and $3 \mathrm{~ms}$, respectively, while the gradient strength was gradually increased up to $70 \%$. Processing and analysis of the spectra was performed usingTopspin 3.5pl7 (Bruker). The signal of the DSS was used as reference to monitor the attenuation of signal intensity. A sharp peak, for either P2R2 or P2R3, was selected and used to observe signal intensity attenuation as a function of gradient strength. The analysis was done by integrationof selected peaks. In conditions where $\Delta \gg \gg \delta_{2}$ and $\delta$, the self-diffusion coefficient, D, was obtained according to the equation ${ }^{174}$ :

$$
\ln (E)=-\gamma^{2} D \delta^{2}\left[\left(\Delta-2 \delta_{2}\right)-\frac{4}{3} \delta\right]\left(g_{2}-g_{1}\right)^{2}
$$


Where $E$ is the diffusion-based signal intensity attenuation, $D$ is the diffusion coefficient, $\gamma$ is the gyromagnetic ratio of protons $\left(\mathrm{g}^{1} \mathrm{H}=267,52221900 \cdot 10^{-6} \mathrm{rad} \cdot \mathrm{s}^{-1} \cdot \mathrm{T}^{-1}\right), \delta_{2}$ was $2 \mathrm{~ms}$ and $\mathrm{g}_{1}, \Delta$ and $\delta$ were constant. The calculation for the hydrodynamic radius $\left(\mathrm{r}_{\mathrm{H}}\right)$ of P2R2 and P2R3 was done using the DSS self-diffusion coefficient $\left(3.7 \cdot 10^{10} \mathrm{~m}^{2} \cdot \mathrm{s}^{-1}\right)$, which was experimentally determined in a separate diffusion NMR experiment, and the $\mathrm{r}_{H}(3.8 \pm$ $0.01 \AA$ ) of DSS. The following relation was used ${ }^{170}$ :

$$
R_{h}^{\text {prot }}=\frac{D_{\text {ref }}}{D_{\text {prot }}}\left(R_{h}^{\text {ref }}\right)
$$

with $D_{\text {ref }}$ and $D_{\text {prot }}$ the self-diffusion coefficients of DSS and P2R2 or P2R3, respectively.

\subsubsection{Solid-state NMR of tau fibrils}

Solid-state NMR (ssNMR) allows the study of proteins, which are not in solution including membrane proteins and amyloid aggregates. Although the basic principles of solid-state and solution-state NMR are the same, the way the information is obtained differs slightly.

\subsubsection{Differences between liquid-state and solid-state NMR}

In ssNMR, the tumbling of the molecule is slower when compared to a molecule in solution. In the spectra this causes broadening of the peaks and decreases spectral resolution ${ }^{175}$. Magic angle spinning (MAS) is a method implemented to improve the resolution of the signals by elimination of chemical shift anisotropy contributions ${ }^{57,175}$ : the sample is inserted into a small rotor (from 4.0 to $0.7 \mathrm{~mm}$ ) and spun at high frequencies on an angle tilted with respect to the $B_{0}$ magnetic field ${ }^{57}$. Similar to solution-state NMR experiments, the insensitivity of ${ }^{13} \mathrm{C}$ and ${ }^{15} \mathrm{~N}$ nuclei can be increased by transfer of magnetization from the more sensitive ${ }^{1} \mathrm{H}$. The cross-polarization (CP) technique allows transfer of magnetization from ${ }^{1} \mathrm{H}$ to insensitive nuclei using RF pulses, by equalizing the energy level between the two different nuclei ${ }^{175}$. The Hartmann-Hahn matching condition requires the rotation frequencies of the spinning sample to be equal to the frequencies of 
two spins for successful transfer of magnetization ${ }^{175}$. By this technique, an enhancement of sensitivity is 4 -fold and 10 -fold for ${ }^{13} \mathrm{C}$ and ${ }^{15} \mathrm{~N}$, respectively. Like in the case of the INEPT block in solution, the $\mathrm{CP}$ is included in many multidimensional SSNMR experiments as initial step in order to obtain magnetization transfer from ${ }^{1} \mathrm{H}$ to ${ }^{13} \mathrm{C}$ or ${ }^{15} \mathrm{~N}$.

\subsubsection{2 ${ }^{13} \mathrm{C}-{ }^{13} \mathrm{C}$ correlation obtained via proton-driven spin diffusion (PDSD)}

Proton-driven spin diffusion (PDSD) experiments are often performed to observe ${ }^{13} \mathrm{C}-{ }^{13} \mathrm{C}$ correlation through dipolar coupling. Equilibrium magnetization is first transferred from ${ }^{1} \mathrm{H}$ to its coupled ${ }^{13} \mathrm{C}$ using cross-polarization (CP) then, during a mixing time period, magnetization is spread via spin diffusion among carbons and protons (figure 2.7) ${ }^{176}$. Depending on the length of the mixing time intra-residual or inter-residual correlation cross-peaks are observed ${ }^{177}$.

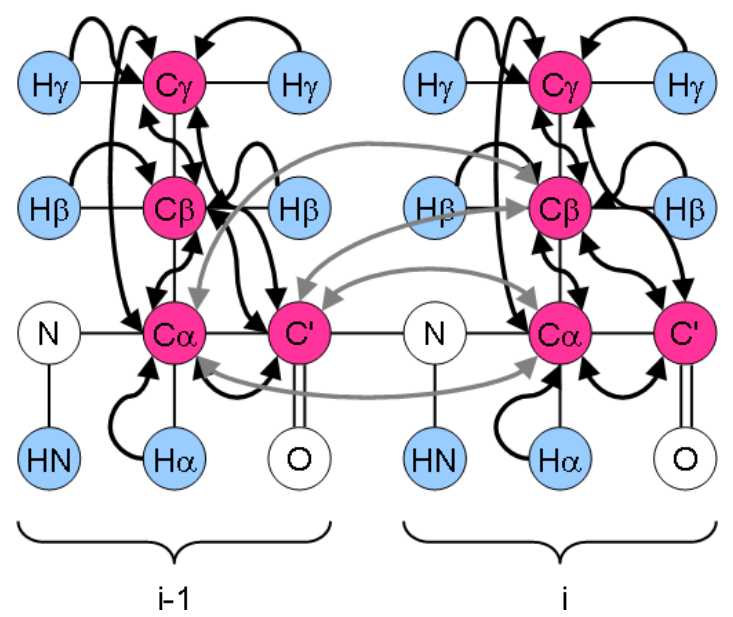

Figure 2.7. Transfer of magnetization in a PDSD experiment.Type of atoms observed in a PDSD experiment together with the path of transferred magnetization. During the mixing time, magnetization is transferred between ${ }^{13} \mathrm{C}$ atoms close in space; protons are also involved in this transfer (black arrows). At longer mixing times, inter-residue transfer of magnetization occurs (grey arrows). 


\subsubsection{2D INEPT transfer-based ${ }^{1} \mathrm{H}^{13} \mathrm{C}$ through-bond correlation}

The strong dipolar coupling between ${ }^{1} \mathrm{H}$ and insensitive nuclei (i.e. ${ }^{13} \mathrm{C}$ and ${ }^{15} \mathrm{~N}$ ) is exploited in CP-based pulse sequences (see section 2.11.9.1) to increase the signal of insensitive nuclei ${ }^{175}$. In contrast, the INEPT sequence (see section 2.11 .2 ) uses scalar couplings to transfer magnetization between coupled spins. Although mostly used in solution-state NMR, the INEPT also finds applications in ssNMR experiments ${ }^{178}$. INEPT-based experiments allow the observation of flexible regions in solid biological samples ${ }^{84,179-181}$ : proton transverse relaxation within rigid regions of solids samples (i.e. in absence of tumbling motion) is faster than the typical delay in the INEPT sequence, therefore only regions maintaining flexibility are detected.

\subsubsection{Preparation of solid-state NMR samples and experimental settings}

Prior to MAS rotor filling, ${ }^{15} \mathrm{~N} /{ }^{13} \mathrm{C}$-labeled fibrils of P2R2 ( $\left.20 \mathrm{mg}\right)$, P2R3 ( $20 \mathrm{mg}$ ) and K32 ( $12 \mathrm{mg}$ ) where ultra-centrifuged in a $45 \mathrm{Ti}$ Ultra Rotor at $127,000 \mathrm{~g}$, at $37^{\circ} \mathrm{C}$ for onehour (Beckman Coulter, Optima MAX XP). This procedure was repeated several times in order to remove residual monomeric protein. DSS (4,4-dimethyl-4-silapentane-1-sulfonic acid) in powder (Sigma-Aldrich) was added to each sample as internal chemical shift reference. PDSD and INEPT experiments were recorded on ${ }^{15} \mathrm{~N} /{ }^{13} \mathrm{C}$-labeled $\mathrm{K} 32$ fibrils packed into a $1.3 \mathrm{~mm}$ rotor. Spectra were acquired at $5{ }^{\circ} \mathrm{C}$ and $11 \mathrm{kHz}$ spinning speed on a Bruker 850 $\mathrm{MHz}$ wide bore spectrometer (Bruker, Germany). The temperature of the spinning sample was calculated from the chemical shift of the bulk water according to the following equation ${ }^{158}$ :

$$
\mathrm{T}=7.83-\delta\left(\mathrm{H}_{2} 0\right) \cdot 96.9 \mathrm{ppm}
$$

A mixing time of $20 \mathrm{~ms}$ was set to obtain intra-residue correlations. The 2D INEPT transferbased ${ }^{1} \mathrm{H}-{ }^{13} \mathrm{C}$ experiments were performed on the same instrument at $5{ }^{\circ} \mathrm{C}$ and $8 \mathrm{kHz}$.

For P2R2 and P2R3, fibrils of uniformly ${ }^{15} \mathrm{~N} /{ }^{13} \mathrm{C}$-labeled peptides were packed into a $3.2 \mathrm{~mm}$ rotor. PDSD and INEPT spectra were recorded at $5{ }^{\circ} \mathrm{C}$ and a spinning speed of $12.5 \mathrm{kHz}$ and $8 \mathrm{kHz}$, respectively, on a Bruker $850 \mathrm{MHz}$ wide bore spectrometer (Bruker, Germany). A mixing time of 20 ms was set to obtain intra-residue correlations in the PDSD spectra. The parameters referring to the ssNMR experiments are reported in table 2.5. 
Spectra were processed using TopSpin 3.5 pl 7 (Bruker, Germany) and analyzed with Sparky ${ }^{154}$ and ccpNMR ${ }^{155}$ software.

Table 2.5. Selected parameters for ssNMR experiments performed on tau fibrils.

\begin{tabular}{|c|c|c|c|c|c|c|c|}
\hline Protein & $\begin{array}{c}\text { Type of } \\
\text { experiment }\end{array}$ & Rotor & \multicolumn{2}{|c|}{ (ppm) } & NS & TD & $\begin{array}{c}\text { Spinning speed } \\
(\mathrm{kHz})\end{array}$ \\
\hline P2R2 & & & ${ }^{13} \mathrm{C}$ & ${ }^{13} \mathrm{C}$ & \multirow{3}{*}{48} & $1274\left({ }^{13} \mathrm{C}\right)$ & \multirow{3}{*}{11} \\
\hline \multirow[t]{4}{*}{ /P2R3 } & PDSD & 3.2 & 299 & 299 & & $1274\left({ }^{13} \mathrm{C}\right)$ & \\
\hline & & & ${ }^{1} \mathrm{H}$ & ${ }^{13} \mathrm{C}$ & & $2048\left({ }^{1} \mathrm{H}\right)$ & \\
\hline & INEPT & 3.2 & 199 & 14 & 160 & $204\left({ }^{13} \mathrm{C}\right)$ & 8 \\
\hline & & & ${ }^{13} \mathrm{C}$ & ${ }^{13} \mathrm{C}$ & \multirow{3}{*}{64} & $1274\left({ }^{13} \mathrm{C}\right)$ & \multirow{3}{*}{12.5} \\
\hline \multirow[t]{3}{*}{ K32 } & PDSD & 1.3 & 299 & 299 & & $1274\left({ }^{13} \mathrm{C}\right)$ & \\
\hline & & & ${ }^{1} \mathrm{H}$ & ${ }^{13} \mathrm{C}$ & & $2048\left({ }^{1} \mathrm{H}\right)$ & \\
\hline & INEPT & 1.3 & 199 & 14 & 208 & $204\left({ }^{13} \mathrm{C}\right)$ & 8 \\
\hline
\end{tabular}




\section{Results: project I}

\subsection{Solution-state NMR of the K32 construct}

The K32 construct comprises the P2 domain and the pseudo-repeat region (from R1 to R4, including the flanking $\mathrm{R}^{\prime}$ domain) of the full-length htau40 (Figure 1.7) ${ }^{52}$. In order to obtain the backbone and side-chain assignment, NMR 2D and 3D experiments were performed on $200 \mu \mathrm{M} \mathrm{K32}$ in $50 \mathrm{mM}$ sodium phosphate buffer at $\mathrm{pH} 6.8$. The ${ }^{1} \mathrm{H}-{ }^{15} \mathrm{~N}$ HSCQ spectrum of K32 displayed little chemical shift dispersion, as previously reported ${ }^{52,56}$ (Figure 3.1a). The resonance assignment of the $2 \mathrm{D}{ }^{1} \mathrm{H}^{-15} \mathrm{~N}$ HSQC (Figure 3.1a) was validated using the assignment previously reported by Mukrasch and collaborators ${ }^{52}$. For the assignment of the $\mathrm{C} \alpha$ and $\mathrm{C} \beta$ resonances in the ${ }^{1} \mathrm{H}-{ }^{13} \mathrm{C}$ HSQC spectrum (Figure $\left.3.1 \mathrm{~b}\right), 3 \mathrm{D} \mathrm{H}(\mathrm{CC})(\mathrm{CO}) \mathrm{NH}-$ TOCSY and $(\mathrm{H}) \mathrm{CC}(\mathrm{CO}) \mathrm{NH}-\mathrm{TOCSY}$ experiments were performed.

a

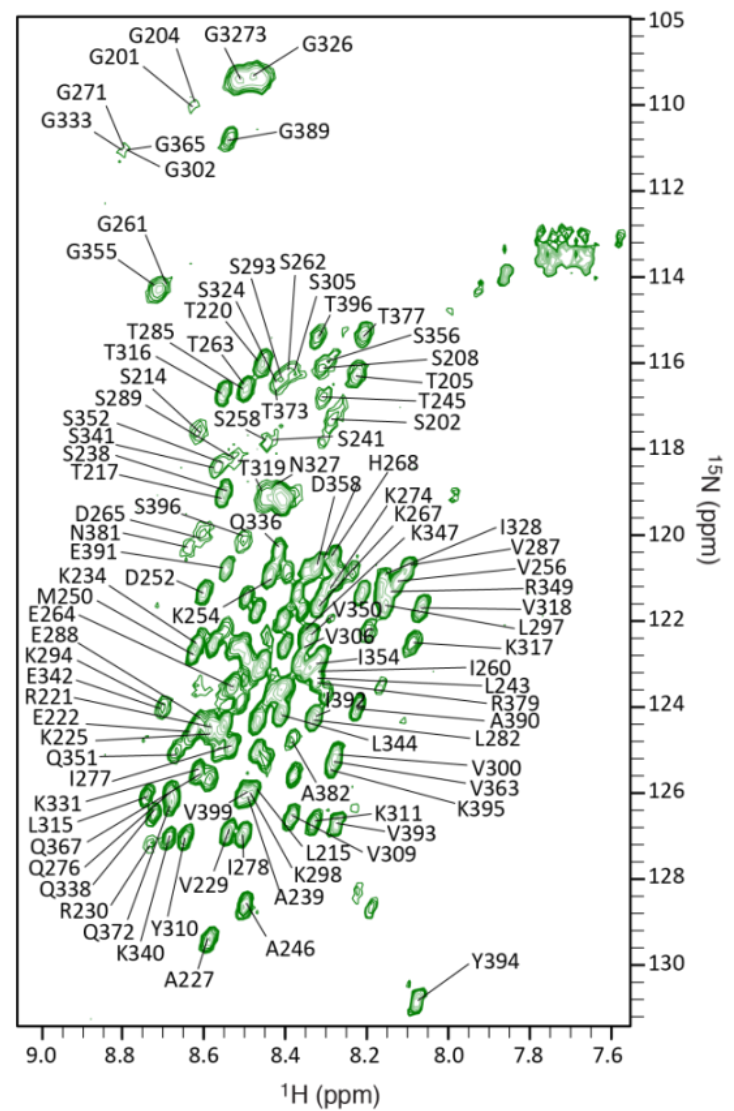

b

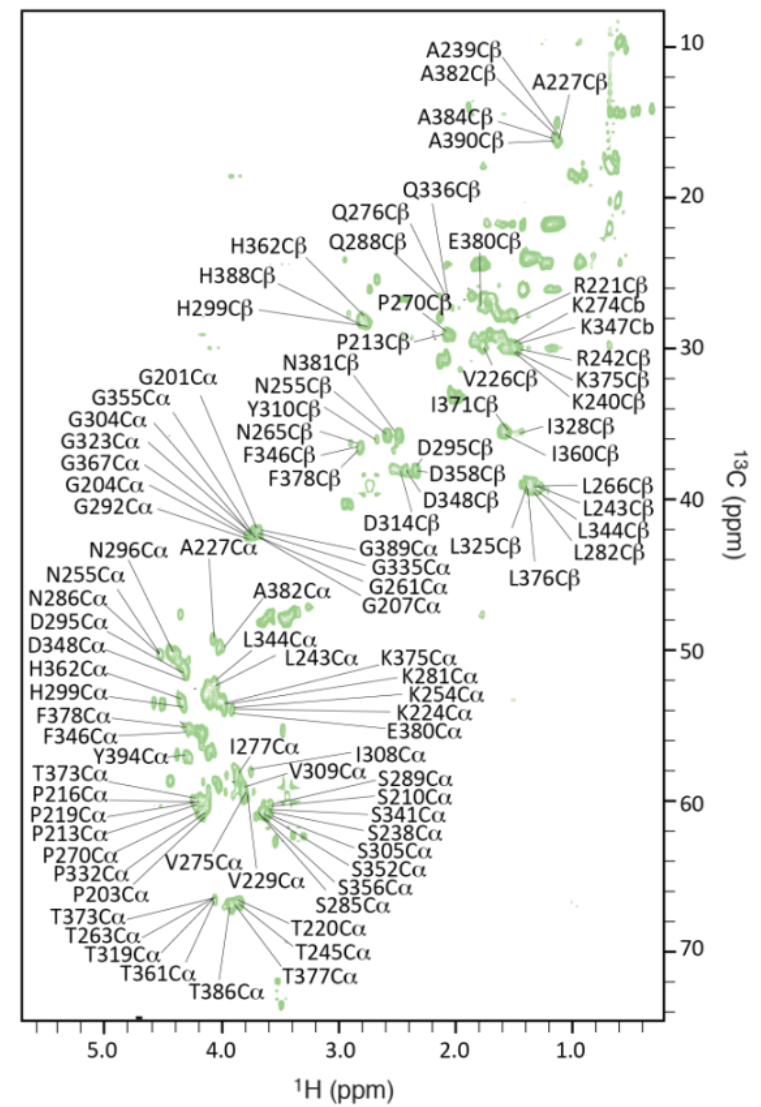

Figure 3.1. Backbone and side chain assignment of $\mathrm{K} 32$ in solution. a) ${ }^{1} \mathrm{H}-{ }^{-15} \mathrm{~N} \mathrm{HSCQ}$ spectrum recorded on the $\mathrm{K} 32$ construct at $5{ }^{\circ} \mathrm{C}$ in $50 \mathrm{mM}$ sodium phosphate buffer ( $2 \mathrm{mM} \mathrm{DTT}, 0.02 \% \mathrm{NaN} 3$ ) at $\mathrm{pH} 6.8 . \mathrm{b}$ ) ${ }^{1} \mathrm{H}-{ }^{13} \mathrm{C}$ HSQC spectrum of $\mathrm{K} 32$ measured at $5{ }^{\circ} \mathrm{C}$ in $50 \mathrm{mM}$ sodium phosphate buffer ( $2 \mathrm{mM} \mathrm{DTT}, 0.02 \% \mathrm{NaN}_{3}$ ) at pH 6.8 .

\subsection{Characterization of in vitro K32 fibrils}


As previously introduced in section 1.4.2, tau is an IDP and shows little tendency to aggregate in vitro. Polyanions, e.g. heparin, induce in vitro tau aggregation by neutralization of the positive charges and thus allow the establishment of hydrophobic interactions between residues from different tau molecules ${ }^{82,182}$. Four independent aggregation assays were performed using $50 \mu \mathrm{M}$ of $\mathrm{K} 32$ in $25 \mathrm{mM}$ Tris- $\mathrm{HCl}$ buffer, at pH 7.4 to give a ssNMR sample. Heparin $\left(\mathrm{M}_{\mathrm{r}} 2 \times 10^{4} \mathrm{~g} / \mathrm{mol}\right.$, Carl Roth $\left.{ }^{\circledR}\right)$ was added to each reaction mixture to a final concentration of $12.5 \mu \mathrm{M}$ (tau-to-heparin molar ratio 4:1). The samples were incubated at $37^{\circ} \mathrm{C}$ for three days to ensure completeness of the reaction. The formation of fibrils was confirmed by measurement of the ThT fluorescence, as shown in figure 3.2a (dark green bar). A reaction mixture containing only the monomeric form of the protein, and no heparin, was also incubated in the same conditions, in order to provide a negative control (Figure 3.2a, light green bar).

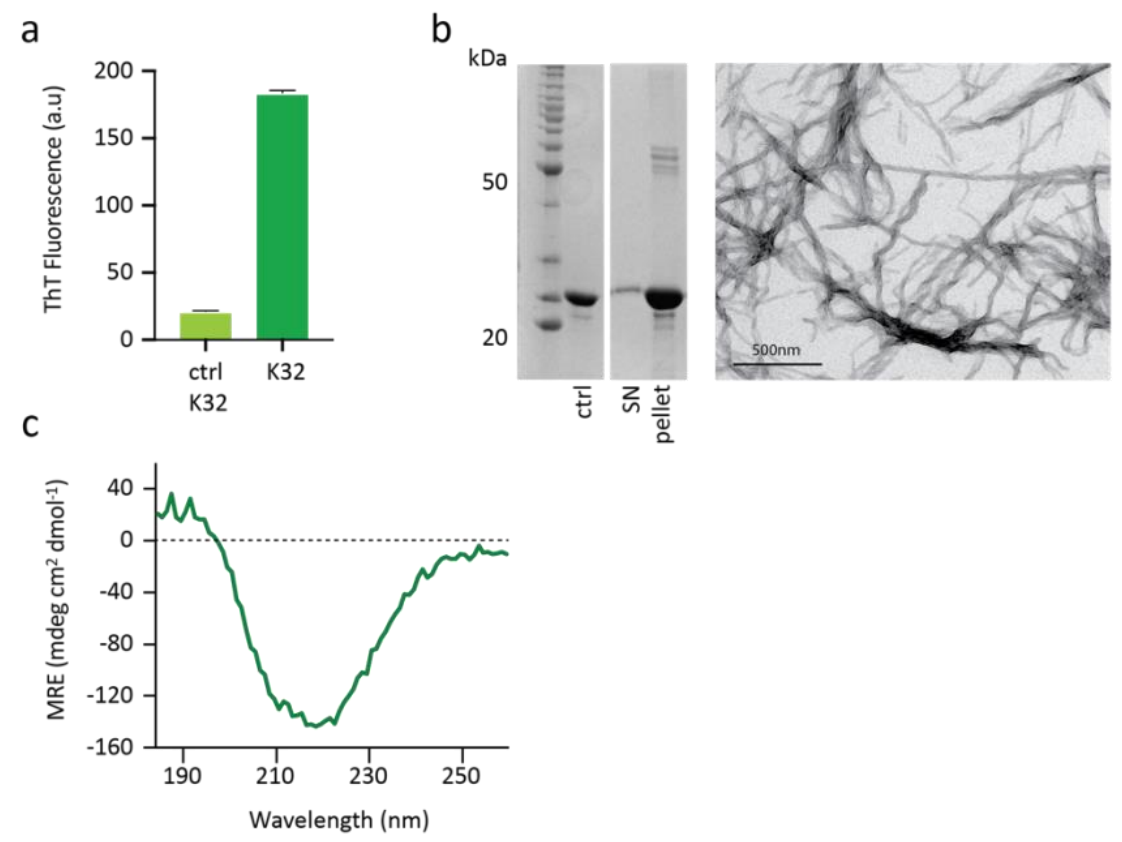

Figure 3.2. Characterization of K32 fibrils. a) ThT fluorescence intensities of the in vitro fibrils obtained from the aggregation of the K32 construct (dark green) in presence of heparin.The ThT intensity for K32 in the monomeric form (light green) was used as control. Error bars indicate the standard deviation over three replicates. b) SDS-PAGE gel of the fibrils of K32 after centrifugation (ctrl, negative control containing only K32 in the monomeric form; SN, supernatant removed after centrifugation). In the right panel, an electron micrograph of the K32 fibril sample, for which ThT fluorescence measurements and SDS-PAGE gel electrophoresis were performed, is shown. Scale bar, $500 \mathrm{~nm}$. c) CD spectrum of the in vitro K32 fibrils; a minimum at $\sim 220 \mathrm{~nm}$ for $\beta$-sheet secondary structure was observed. 
To quantify the amount of formed fibrils, the samples were ultracentrifuged, followed by separation of the pellet from the supernatant. Each fraction was subsequently loaded onto an SDS-PAGE gel to check the protein composition. After the gel electrophoresis run, $\sim 90 \%$ of the protein was present in the pellet, and $\sim 10 \%$ remained in the supernatant (Figure 3.2b, left panel).

Next, the pellet retrieved after ultracentrifugation was resuspended in deionized water and used for acquisition of a CD spectrum. The CD spectrum showed a minimum at $\sim 220 \mathrm{~nm}$, indicative of $\beta$-sheet structure (Figure 3.2c). The presence of fibrils in the pellet was further confirmed by electron microscopy: twisted fibrils were observed in the electron micrograph (Figure 3.2b, right panel).

\subsection{Proton Driven Spin Diffusion (PDSD) experiment on K32 fibrils}

The focus of this project was to understand the involvement of the proline-rich region in the formation of tau fibrils and/or their structural rearrangement. To this end, ssNMR Proton Driven Spin diffusion (PDSD) experiments were performed on uniformly ${ }^{13} \mathrm{C} /{ }^{15} \mathrm{~N}$ labeled in vitro $\mathrm{K} 32$ fibrils using 20 ms mixing time. Prior to the measurement, the fibrils had been ultracentrifuged (to remove residual monomeric protein) and were packed into a $1.3 \mathrm{~mm}$ magic angle spinning (MAS) rotor.

As shown in figure 3.3 , the resulting spectrum displayed signal overlap and few isolated cross peaks. Using the chemical shift values for amino acids in $\beta$-sheet conformation ${ }^{183}$, the residue type could be identified for some cross peaks, e.g. serine, threonine and proline residues, but no specific assignment could be performed. To more specifically determine the identity of some of the cross peaks, the spectrum obtained for the fibrils of K19 were superimposed on the spectrum of K32 fibrils (Figure 3.4b, K19 in black). The resonances assignment of the spectrum of K19 was previously reported by Xiang and collaborators ${ }^{96}$.

The K19 sequence contains the R1, R3 and R4 pseudo-repeats of tau (Figure 3.4a), while the native cysteine residue in $\mathrm{R} 3, \mathrm{C} 322$, had been mutated to alanine in order to avoid the formation of intra-molecular disulfide bridges. This mutation is not present in the sequence of the K32 construct, thus the cross peak of C322A, which in K19 appears 
separated from the rest of the resonances, was not matching with any of the cross peaks in the PDSD spectrum of K32 (Figure 3.4b).

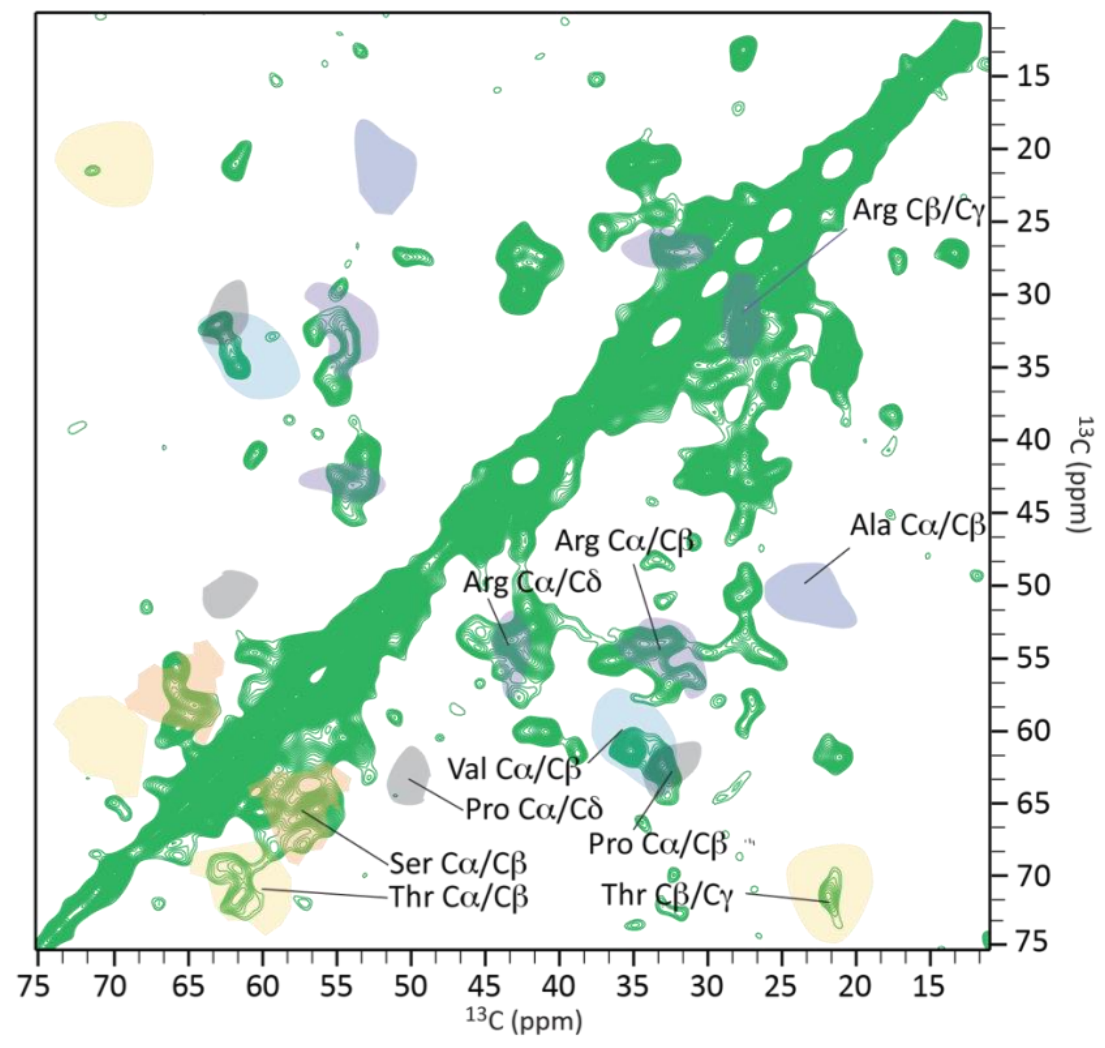

Figure 3.3. PDSD spectrum of $\mathbf{K} 32$ fibrils. Regions corresponding to the ${ }^{13} \mathrm{C}$ resonances of selected amino acids types in $\beta$-sheet conformation reported by Fritzsching et al., J Biomol NMR 2013.

Figure 3.4 shows that a larger number of cross peaks is visible in the 2D PDSD spectrum of K32 when compared to that of K19. In particular, more $\mathrm{C} \alpha / \mathrm{C} \beta$ cross peaks are present in the threonine and serine region of the $\mathrm{K} 32$ spectrum. The higher number of cross peaks in these regions is consistent with the relative abundance of these amino acid types: K32 contains 12 threonine and 22 serine residues, while K19 only contains 4 threonine and 8 serine residues. Because only rigid residues are detected in the PDSD spectrum, the comparison shown in figure 3.4 further indicates that the $\mathrm{K} 32$ fibrils contains more cross- $\beta$ structure when compared to K19 fibrils. This interpretation is based on the assumption that the larger number of cross-peaks is not purely caused by a much larger structural heterogeneity of K32 fibrils when compared to K19 fibrils.

For several residues belonging to the repeats $\mathrm{R} 1$ and $\mathrm{R} 3$, cross peaks between the two spectra matched (Figure 3.4). For example, the cross peaks assigned to S262 (R1) and 
S316/S320 (R3) in K19 matched with cross peaks in the spectrum of K32. A similar observation was made for the cross peaks assigned to I260 (R1), as well as I308 and I328 (R3). The comparison suggests that residues belonging to the repeats $R 1$ and $R 3$ are rigidified upon fibrillization of both K19 and K32.

a

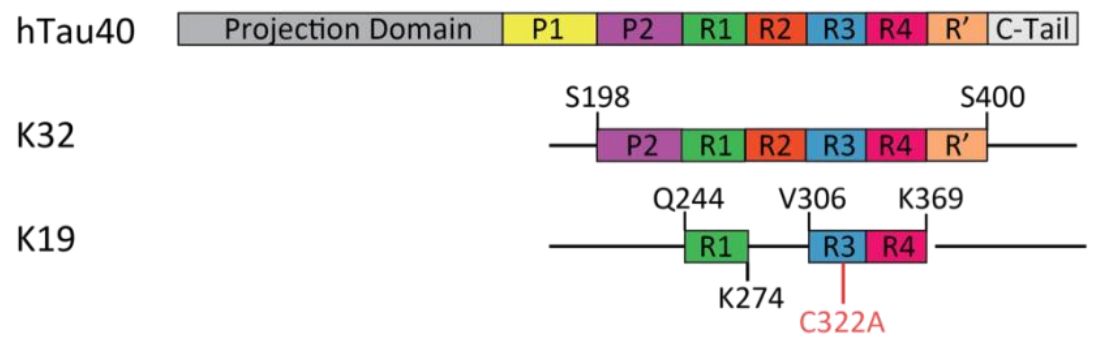

b

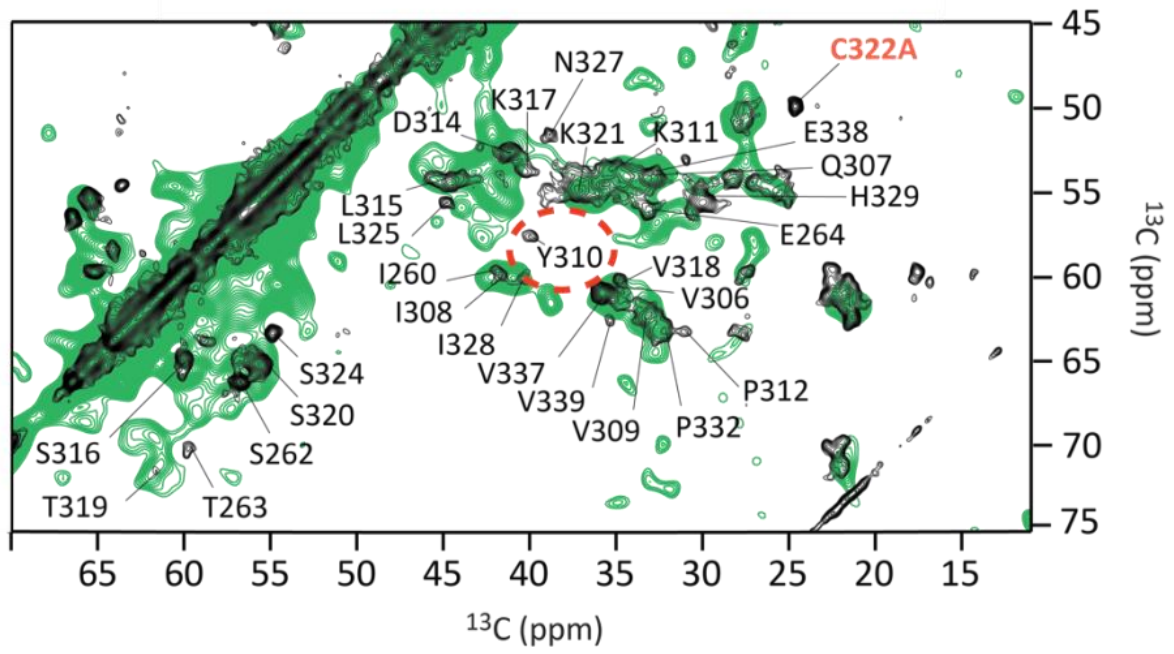

Figure 3.4. Analysis of the PDSD spectrum of K32 fibrils. a) Schematic representation of htau40, K32 and K19 domain organization. K19 has the mutation C322A to avoid intermolecular disulfide bond formation. b) Superposition of PDSD spectra of K32 (green) and K19 (black) fibrils.

K19 contains a single tyrosine, Y310, which is located in repeat R3. In the 2D PDSD spectrum, the $C \alpha / C \beta$ cross peak of $Y 310$ is well separated from other signals, as shown in figure 3.4. For K32, two cross peaks appeared in the spectrum in the same region, but could not be unambiguously assigned since two tyrosine residues, Y310 (in R3) and Y394 (in R'), are present in its sequence. In addition, the two potential tyrosine cross peaks could originate from a single tyrosine in two different fibril conformations. Nevertheless, the crowding in the $\mathrm{K} 32$ spectrum and the lack of 3D experiments suggested that a different approach was needed to better understand to which extent the proline-rich region contributes to the fibrils. 


\subsection{Detection of the flexible regions in K32 fibrils}

Approaching the issue from a different perspective, a 2D INEPT-based ${ }^{13} \mathrm{C}-{ }^{13} \mathrm{C}$ correlation experiment was recorded on the sample of K32 fibrils. As previously mentioned in the methods section 2.11.9.3, this experiment allows the detection of the flexible regions in a solid sample based on their different mobility ${ }^{181}$.

In the transition from random coil to $\beta$-sheet conformation, the amino acid residues included in the fibril core lose flexibility and become invisible in an INEPT-based ${ }^{13} \mathrm{C}-{ }^{13} \mathrm{C}$ correlation experiment. Thus, the ssNMR INEPT and the solution state ${ }^{1} \mathrm{H}-{ }^{13} \mathrm{C}$ HSQC spectra were superimposed, with the purpose to reveal the tau residues that are not part of the rigid core of $\mathrm{K} 32$ fibrils. Upon superposition of the spectra, the cross peaks corresponding to the $C \alpha / C \beta$ resonances of proline, glycine, threonine and serine residues matched (Figure 3.5). Most of these residues are located in the proline-rich region, which is considered to be part of the fuzzy coat of the fibrils ${ }^{83}$. Thus, their visibility in the INEPT spectrum could be explained by their higher degree of mobility. The analysis of the domains considered to be in the rigid core of the fibrils was complicated by the signal overlap of the ssNMR INEPT spectrum, as well as the clustering of the $C \alpha / C \beta$ resonances in the ${ }^{1} \mathrm{H}-{ }^{13} \mathrm{C}$ HSQC. In the $\mathrm{C} \alpha$ region of the INEPT spectrum, the signal intensity of the residues in the two hexapeptides ${ }^{275}$ VQIINK ${ }^{280}$ and ${ }^{306}$ VQIVYK $^{311}$ in R2 and R3, respectively, was attenuated (Figure 3.6b). In the $C \beta$ region of the spectrum, more isolated cross peaks, e.g. those of N265, Q276 and H299 located in the R1 domain, D314, N327 and Q336 in the R3 domain, as well as H338, F346, D348, Q351 and H362 in the R4 domain, were broadened beyond detection in the sSNMR INEPT spectrum (Figure 3.6a). Consistent with the PDSD spectrum, this suggested that the R1, the R3 and the R4 domains are included in the core of $\mathrm{K} 32$ fibrils. 


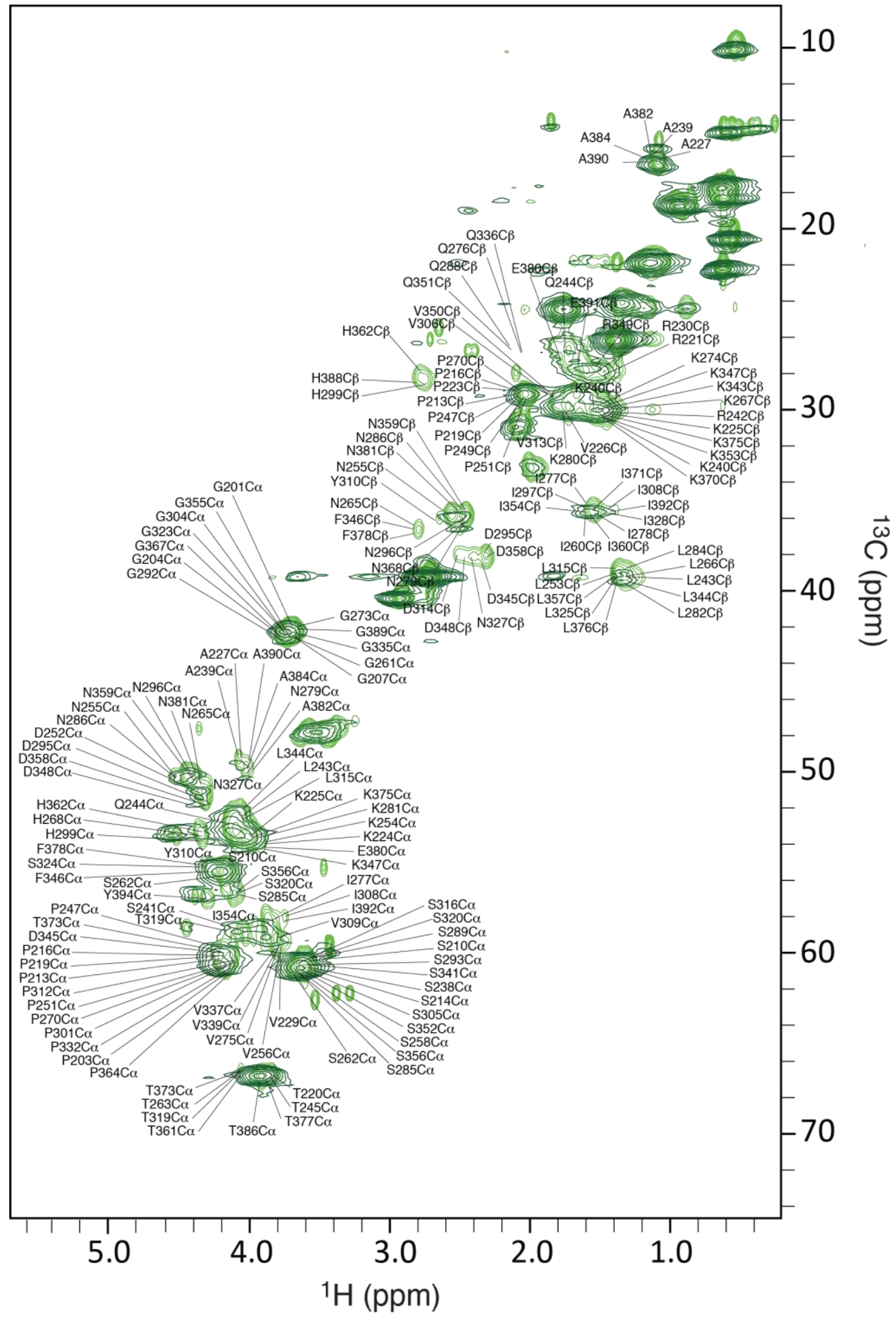

Figure 3.5 Detection of the flexible regions in K32 fibrils. Superposition of the ${ }^{1} \mathrm{H}-{ }^{13} \mathrm{C} \mathrm{HSQC} \mathrm{(light} \mathrm{green)} \mathrm{and}$ the INEPT (dark green) of monomeric and fibrillized K32, respectively. Regions adopting a $\beta$-sheet conformation as a result of the fibril formation cannot be detected in the INEPT-based SSNMR experiment.The assignments shown refers to the $C \alpha$ and $C \beta$ atoms in the monomeric form of the peptide. 
Regarding the $\mathrm{P} 2$ domain and the ${ }^{225} \mathrm{KVAVVRT}^{231}$ sequence, signal intensity loss was observed for V226, A227, V228 and V229 (Figure 3.6c), although the cross peaks did not completely disappear. Thus, a partial recruitment of the P2 domain in the fibrils of K32 is likely.
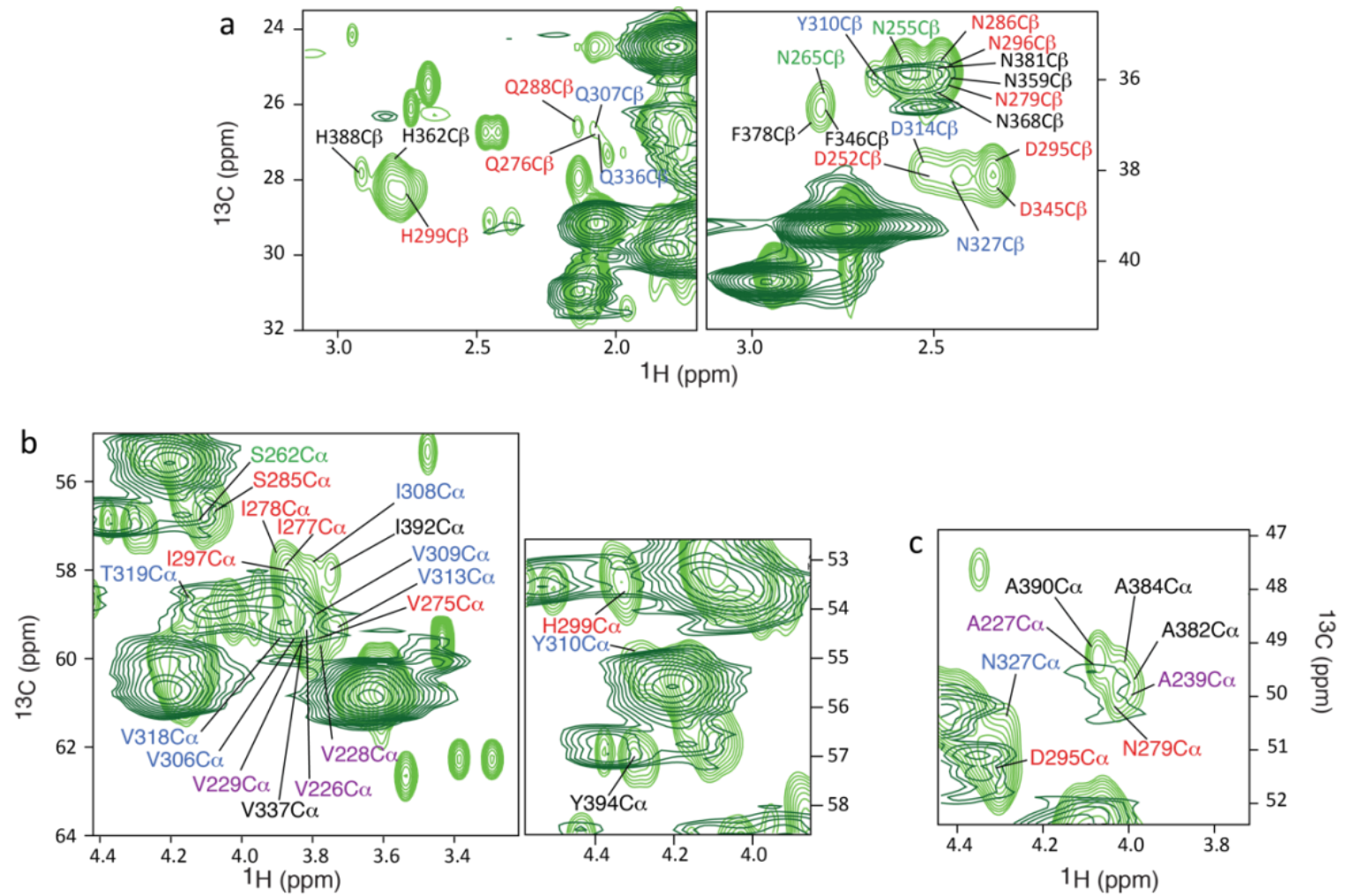

Figure 3.6. Selected regions of the sSNMR INEPT spectrum of K32. Superposition of the ${ }^{1} \mathrm{H}-{ }^{13} \mathrm{C} \mathrm{HSQC}$ (light green) and the INEPT (dark green) of monomeric and fibrillized K32, respectively. a) Portions of the C $\beta$ region of the spectra showing disappearing residues belonging to the R1 (green labels), the R2 (red labels) and the R3 (blue labels) domains. b) Portions of the C $\alpha$ region showing residues of the R2 (red labels) and R3 (blue labels) experiencing signal intensity attenuation. c)Portion of the C $\alpha$ region showing the alanine cluster with signal intensity attenutation: A227 and A239 in the P2 domain (purple labels) are also affected. 


\subsection{Preparation of the $\mathrm{P} 2 \mathrm{R} 2$ and the $\mathrm{P} 2 \mathrm{R} 3$ polypeptides for a simplistic model of tau fibrils}

The sequences P2R2 and P2R3 were used as simplified models of tau, because they combine one proline-rich region (P2: residues S198-Q244) with one pseudo-repeat (R2: residues V275-S305; or R3: residues V306-V336) and thus represent two important sequence properties of the full-length htau40 protein (Figure 2.1, material and methods). To avoid formation of intermolecular dimers, the native cysteine residues, i.e. C291 (R2) and C322 (R3), were substituted by alanine.

The peptides where produced recombinantly in E.coli as described in section 2.2.5. Optimal purity of the samples was achieved with a final size exclusion chromatography: fractions free from contaminants (Figure 3.7) were selected to achieve very high sample purity.
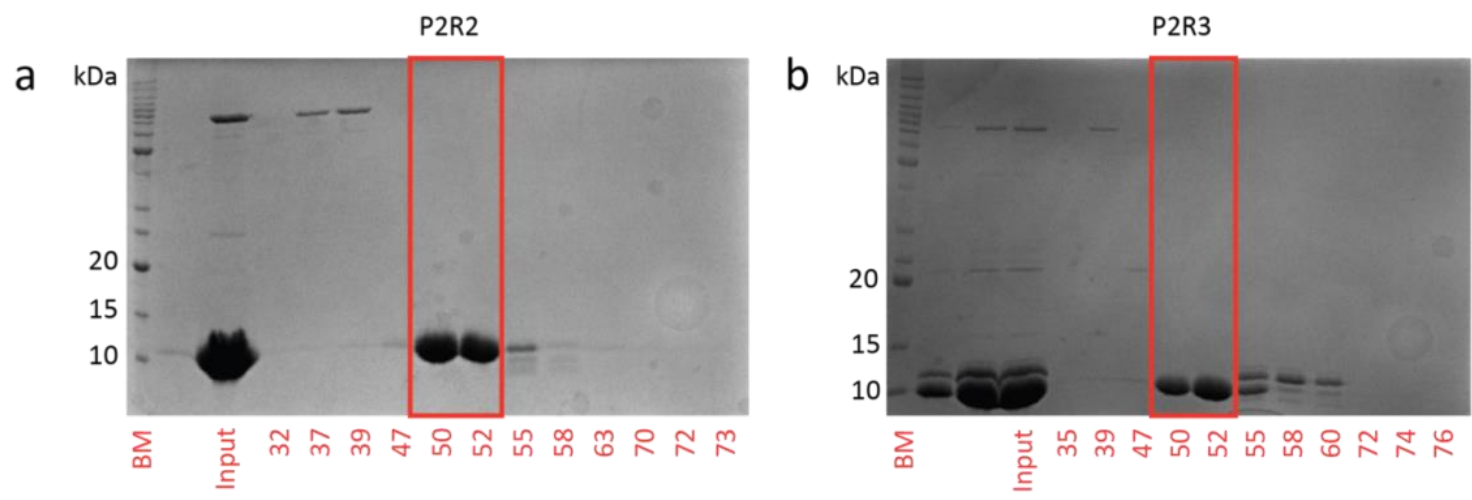

Figure 3.7 Purification of P2R2 and P2R3 polypeptides by size exclusion chromatography. SDS-PAGE gels of a) P2R2 and b) P2R3 size exclusion chromatography fractions. Indicated in the red box are the fractions with the highest purity, which were selected. Lane BM, protein marker (Benchmark TM); lane input, sample before the size exclusion chromatography.

\subsection{Characterization of the intrinsically disordered properties of P2R2 and P2R3}

2D ${ }^{1} \mathrm{H}-{ }^{15} \mathrm{~N}$ HSCQ spectra were acquired on either P2R2 or P2R3 in $50 \mathrm{mM}$ sodium phosphate buffer at $\mathrm{pH}$ 6.8. The resulting spectra displayed little chemical shift dispersion, ranging from $\sim 8.0$ to $\sim 8.8 \mathrm{ppm}$ (Fig. 3.8a and b). 

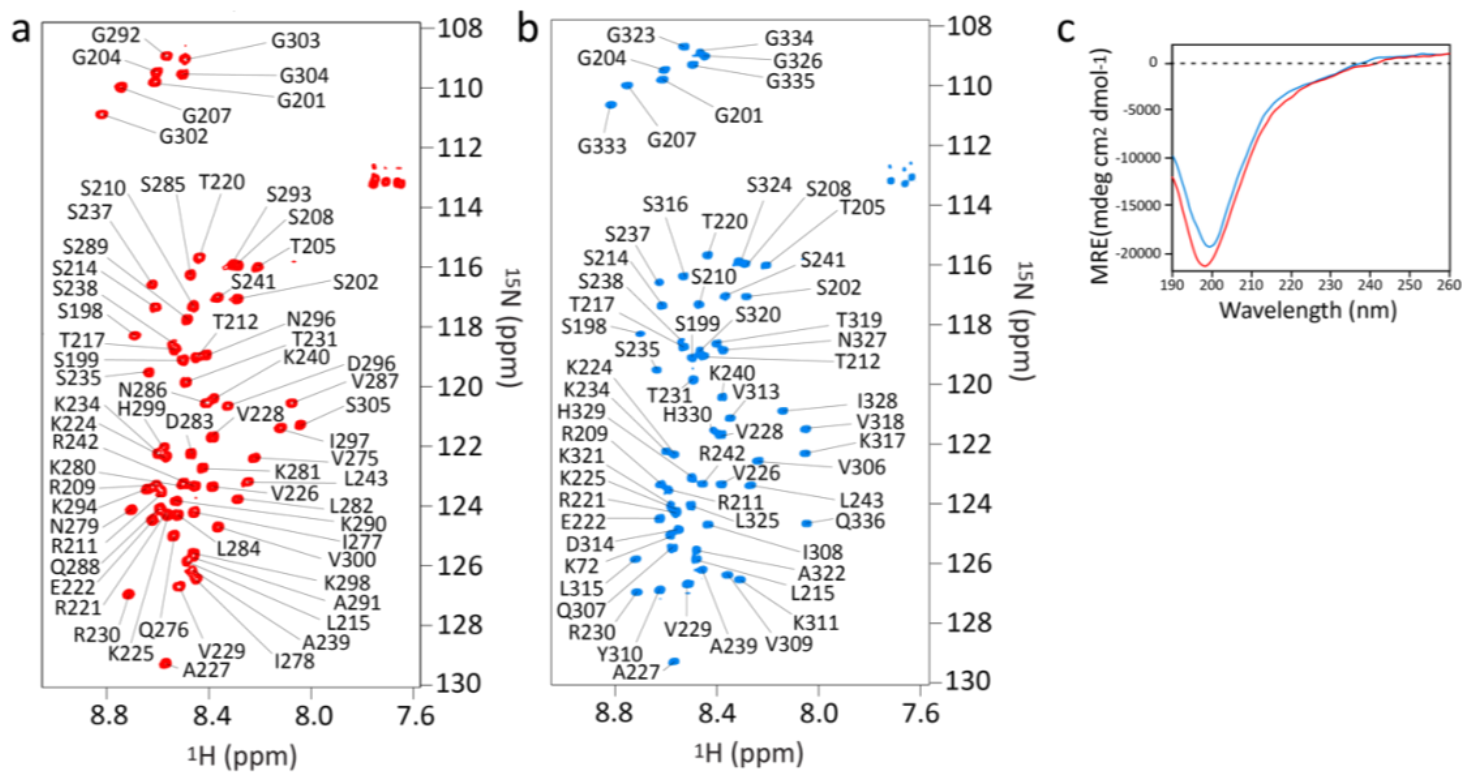

Figure 3.8. Intrinsically disordered properties of the $P 2 R 2$ and $P 2 R 3$ polypeptides. a-b) ${ }^{1} \mathrm{H}-{ }^{15} \mathrm{~N} H S C Q$ spectra of P2R2 (red) and P2R3 (blue) with their respective resonance assignments. c) Circular dichroism spectra of $100 \mu \mathrm{M}$ P2R2 (red) and $100 \mu \mathrm{M}$ P2R3 (blue) in the monomeric form: a minimum $200 \mathrm{~nm}$ is indicative of random coil conformation.

Next, 3D NMR experiments were performed, in order to obtain the backbone resonance assignment of P2R2 and P2R3 (Figure 3.8a and b). The assignment was further validated through comparison with the backbone resonance assignment of htau40 (BMRB entry doi:10.13018/BMR17920).

Calculation of the $C \alpha$ chemical shift difference $(\Delta C \alpha)$ between htau40 and either P2R2 or P2R3 (Figure 3.9a and b) showed differences $<\sim 0.4 \mathrm{ppm}$, with the exception for the region surrounding the substitutions C291A (P2R2) and C322A (P2R3) and the residues at the $\mathrm{C}$ - and $\mathrm{N}$-terminus of each sequence. The analysis demonstrated that the local conformational properties within each of the domains (P2, R2, R3) of the two peptides in solution are similar to those of full-length tau. 
a

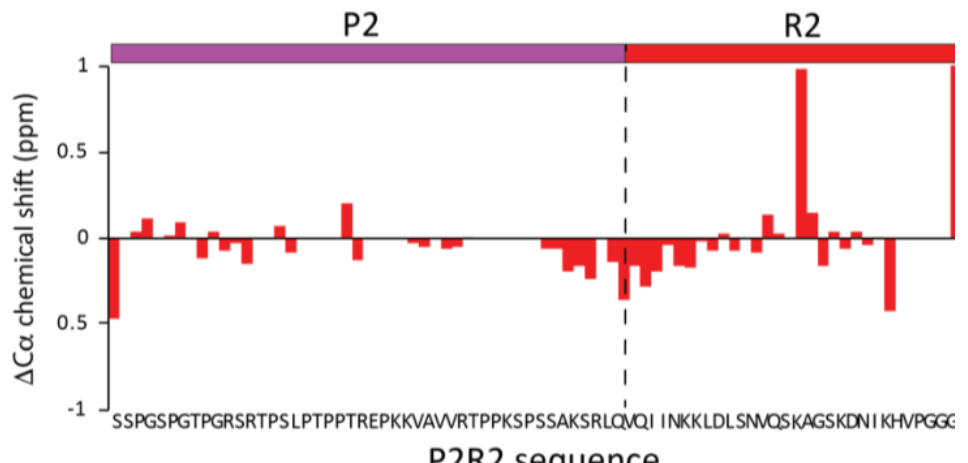

P2R2 sequence

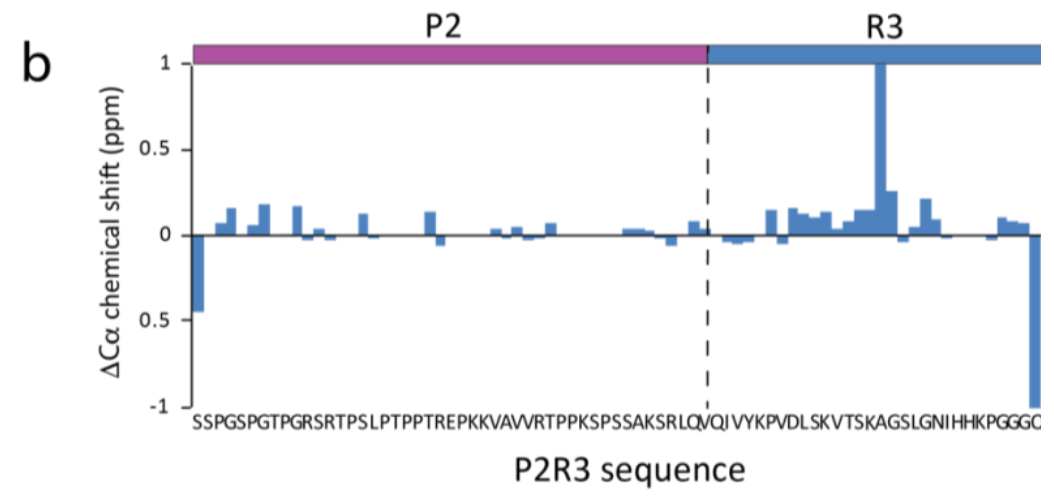

Figure 3.9. $\mathrm{C} \alpha$ chemical shift difference between htau40 and P2R2 or P2R3. The C $\alpha$ chemical difference was calculated for htau40 and either P2R2 (panel a, red) or P2R3 (panel $b$, blue). The strongest chemical shift perturbation occured in the regions surrounding the cysteine residues substituted with alanine, C291A and C322A respectively.

The intrinsically disordered properties of P2R2 and P2R3 were further investigated using CD and diffusion NMR. CD spectra, acquired on $100 \mu \mathrm{M}$ of P2R2 and P2R3 displayed a minimum at $\sim 200 \mathrm{~nm}$, indicative for random coil conformation (Figure 3.8c). Next, diffusion NMR experiments were performed to obtain molecular size information ${ }^{171,174}$. Hydrodynamic radii $\left(R_{H}\right)$ were obtained by measuring the self-diffusion coefficients $(D)$ on $1 \mathrm{mM} \mathrm{P2R2/P2R3} \mathrm{in} 50 \mathrm{mM}$ sodium phosphate buffer at pH 6.8 (Figure 3.10a and b). For spectral referencing and calculation of hydrodynamic radii, $1 \mathrm{mM}$ DSS was added freshly to the samples.

Diffusion coefficient values obtained for P2R2 and P2R3 were $5.2637 \pm 1.95 \times 10^{-11} \mathrm{~m}^{2} \mathrm{~s}^{-1}$ and $5.4929 \times 10^{-11} \pm 1.96 \mathrm{~m}^{2} \mathrm{~s}^{-1}$, respectively. Using equation 10 (see material and methods, section 2.11.8.1), values of $27.2 \pm 0.24 \AA$ and $25.9 \pm 0.33 \AA$ were calculated for P2R2 and P2R3, respectively. The $R_{H}$ values obtained for P2R2 and P2R3 were consistent with values obtained for peptides in random coil conformation or highly denatured proteins reported in literature ${ }^{174,184}$. In a study reporting predicted values of $R_{H}$ for intrinsically disordered proteins of different lengths, peptides of $\sim 70-80$ amino acids displayed similar values to those obtained for P2R2 and P2R3 ${ }^{171}$. This suggested that the two peptides maintain their 
monomeric form in solution. The combined data indicated that P2R2 and P2R3 display intrinsically disordered properties similar to full-length htau40.
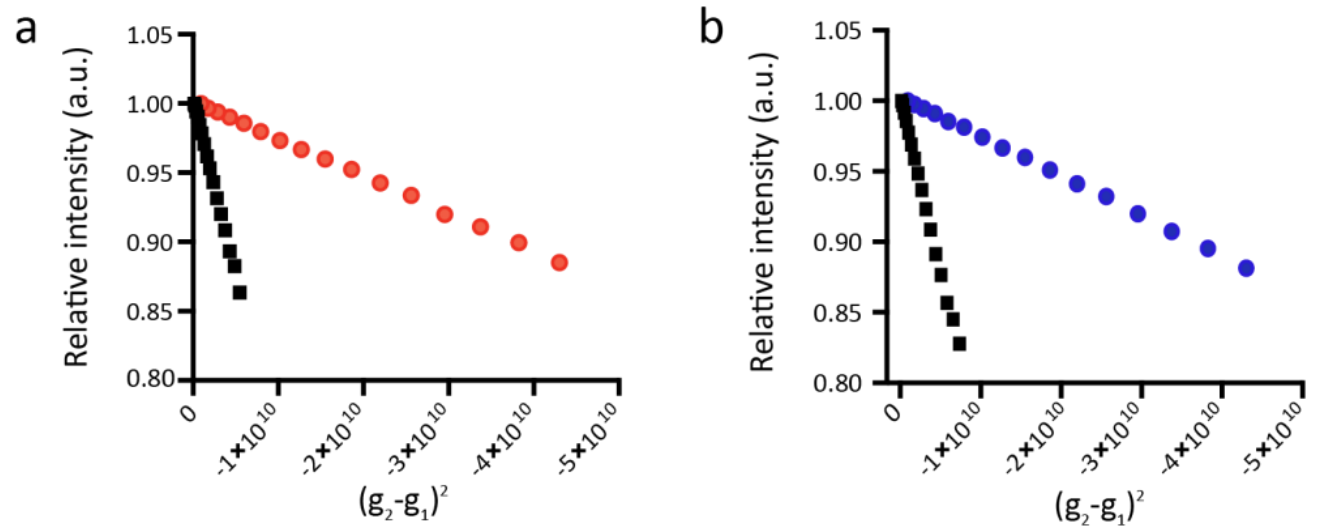

Figure 3.10. Decay of NMR signals of P2R2 and P2R3 in NMR diffusion experiments. NMR diffusion experiments recorded on $1 \mathrm{mM}$ of either P2R2 (a, red) or P2R3 (b, blue) at $5{ }^{\circ} \mathrm{C}$, in $50 \mathrm{mM}$ sodium phosphate buffer, $\mathrm{pH}$ 6.8. For either P2R2 or P2R3 a sharp signal at $0.89 \mathrm{ppm}$ was selected. The relative intensity for the selected signal is plotted against the gradient field strength, $\left(g_{2}-g_{1}\right)^{2}$, and is compared to the diffusion rate of $1 \mathrm{mM}$ of DSS (black).

\subsection{In vitro P2R2 and P2R3 fibrillization}

In order to produce in vitro fibrils, heparin was added to $200 \mu \mathrm{M}$ P2R2/P2R3 in $25 \mathrm{mM}$ Tris-HCl buffer at pH 7.4 to a final concentration of $50 \mu \mathrm{M}$ (1:4 heparin:peptide ratio) ${ }^{182}$. Similar to K32, ThT fluorescence measurements were used to monitor the fibrillization reaction (Figure 3.11a). Fibrils of P2R2 and P2R3 displayed differences in the ThT fluorescence intensities, suggesting either that the P2R2 peptide had a decreased efficiency in the fibrils formation or that its fibrils interacted differently with the amyloid dye. The amount of protein which aggregated into fibrils was similar for the two peptides, as shown in the picture of the SDS-PAGE gel of the pellet and the supernatant fractions (Figure 3.11c). This observation indicated that the weaker ThT intensity observedin case of P2R2 was not caused by inefficient fibrillization. Because the intensity of ThT fluorescence is influenced by the conformation of the fibrils with which it interacts ${ }^{185}$, it rather suggested that the fibrils of P2R2 have a different conformation when compared to P2R3 fibrils. 
After resuspension in deionized water, the pellet was used for CD measurements. The resulting spectra showed minima indicative for $\beta$-sheet structure, however this was more pronounced for P2R3 than for P2R2, which displayed minima at $\sim 220 \mathrm{~nm}$ and $\sim 210$ $\mathrm{nm}$, respectively (Figure 3.11b). Consistent with the ThT fluorescence data, the CD spectra highlighted a difference in the conformation of P2R2 and P2R3 fibrils. This was further supported by electron microscopy performed on the fibrils: a more twisted morphology was observed for P2R3 fibrils, while in P2R2 fibrils resembled straight filaments (Figure 3.11d).
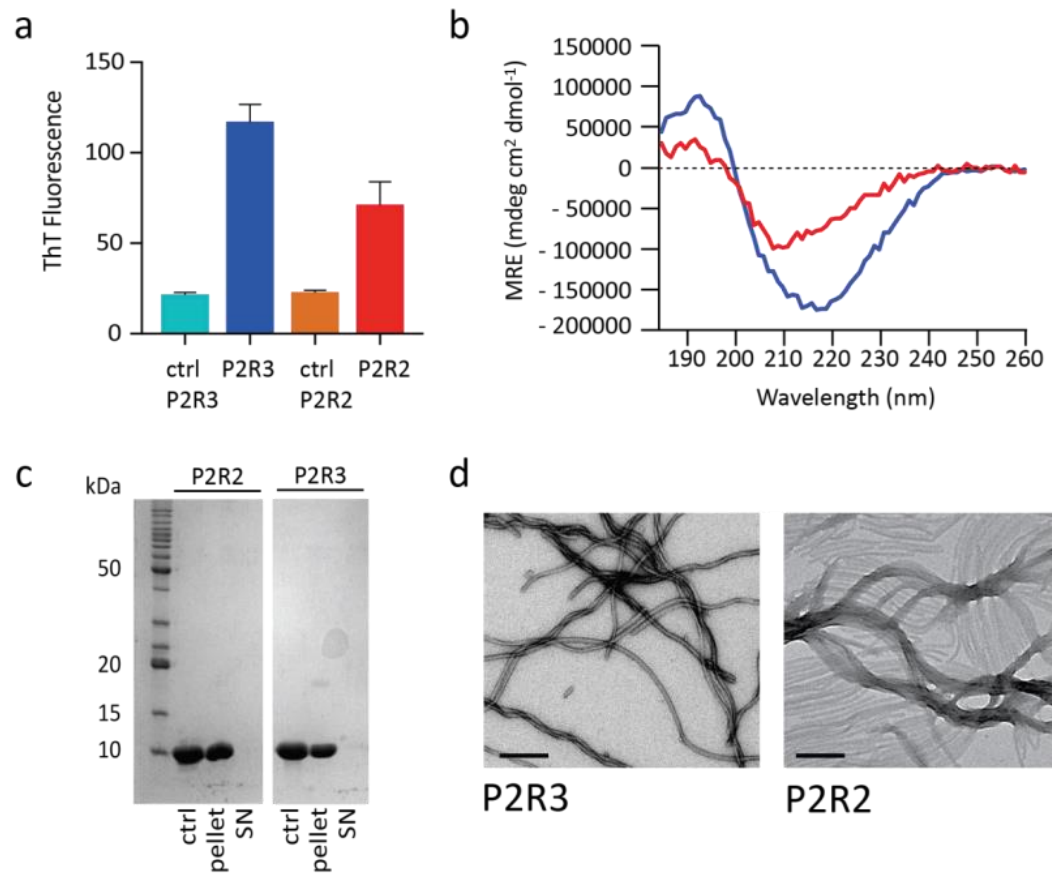

d

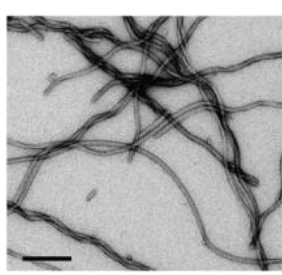

P2R3

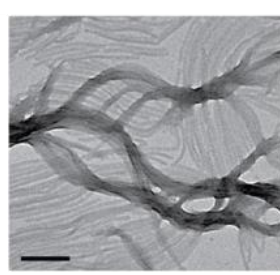

P2R2

Figure 3.11. Characterization of the in vitro fibrils of P2R2 and P2R3. a) ThT fluorescence intensities of P2R2 (red), P2R3 (blue) fibrils. Data for the peptides in the monomeric form are also shown: P2R2 (orange) and P2R3 (light blue). Error bars indicate the standard deviation over three sample replicates. b) CD spectra of the in vitro fibrils of P2R2 (red) and P2R3 (blue). c) SDS-PAGE gel of the fibrils of P2R2 and P2R3 after centrifugation (ctrl, negative control containing only P2R2 or P2R3 in the monomeric form; SN, supernatant removed after centrifugation). d) Electron micrographs of P2R2 (right) and P2R3 (left) fibrils. Scale bars, 500 $\mathrm{nm}$.

\subsection{PDSD experiments on P2R2 and P2R3 fibrils}

Following a similar approach as for the K32 construct, 2D PDSD experiments were performed on uniformly ${ }^{13} \mathrm{C} /{ }^{15} \mathrm{~N}$-labeled P2R2 and P2R3 fibrils. Because the amount of 
fibrils was higher when compared to $\mathrm{K} 32$, a $3.2 \mathrm{~mm}$ rotor was used for the measurements. The resulting spectra displayed good resolution and the cross peaks were more isolated, most likely because of the decreased number of residues in the peptides when compared to K32 (Figure 3.12a and b).
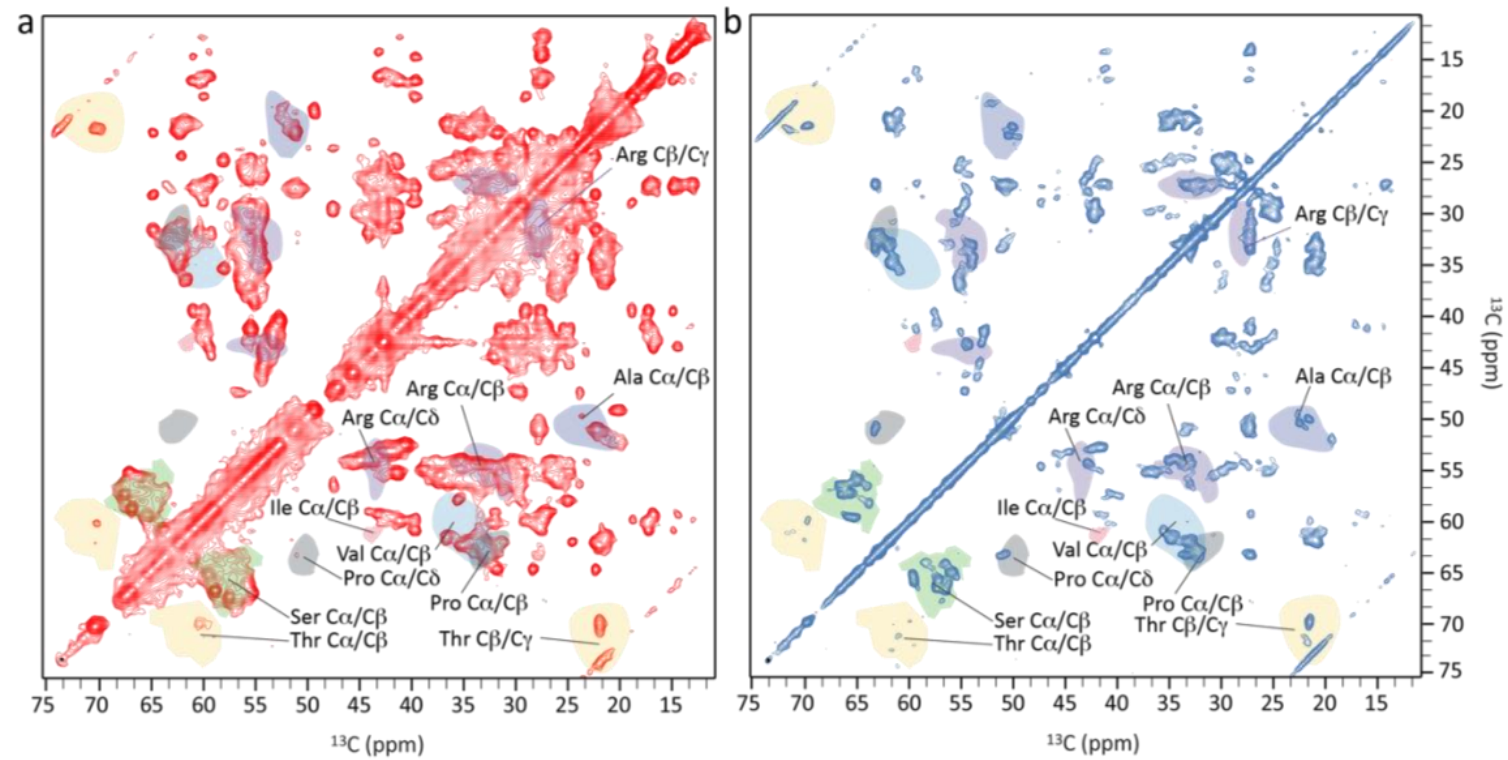

Figure 3.12. PDSD spectra of $P 2 R 2$ and $P 2 R 3$ fibrils. a-b) PDSD spectra of in vitro fibrils of $P 2 R 2$ (red) and P2R3 (blue). The ${ }^{13} \mathrm{C}$ chemical shifts of the different amino acid types in $\beta$-sheet conformation reported by Fritzsching et al., J Biomol NMR 2013 are indicated in different colors.

In the spectra of both peptides, signals appeared in the region corresponding to the $C \alpha / C \beta$ resonances of threonine residues. Moreover, one of the P2R2 cross peaks observed in the threonine $C \alpha / C \beta$ region above the diagonal superimpose with cross peaks of P2R3 fibrils, suggesting that they originate from the same two threonine residues (Figure 3.13). Notably, only one threonine residue is present in the R3 domain, i.e. T319, while the R2 domain contains none. Thus, the two superimposing cross peaks in the PDSD spectra of $\mathrm{P} 2 \mathrm{R} 2$ and $\mathrm{P} 2 \mathrm{R} 3$ respectively might originate from the $\mathrm{P} 2$ domain, which contains five threonine residues. 
a

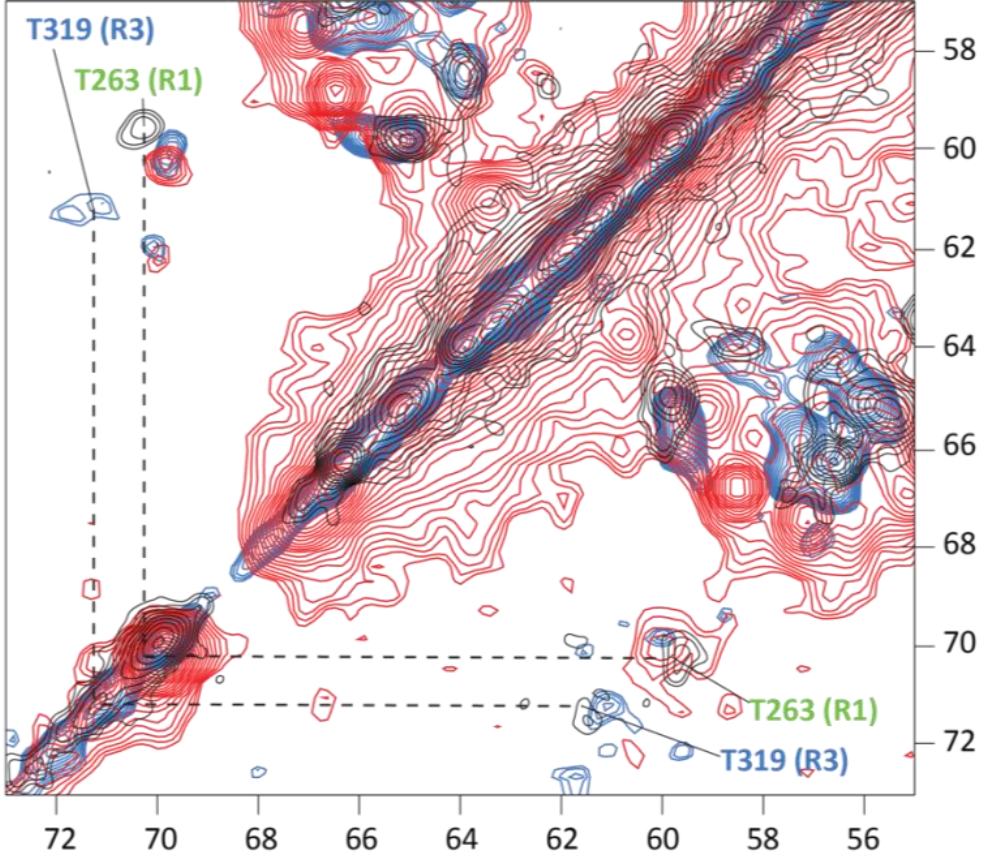

b

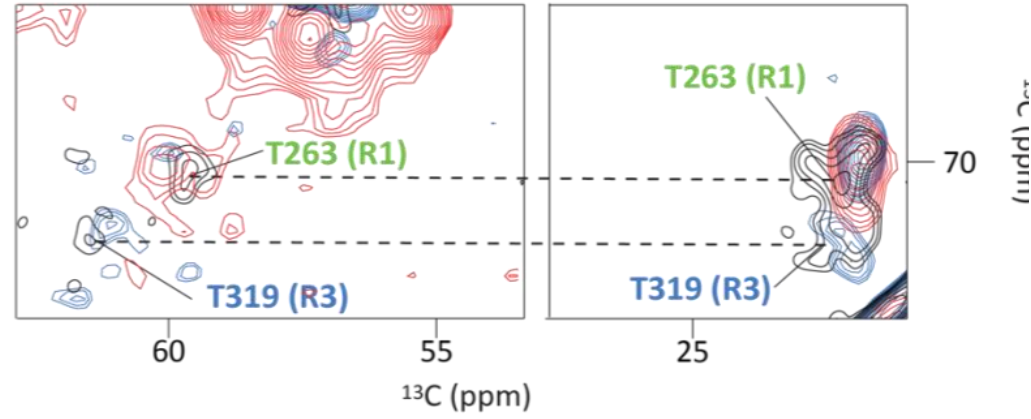

Figure 3.13. The threonine resonance region in the PDSD spectra of P2R2 and P2R3.a) Superposition of the threonine $C \alpha / C \beta$ and b) $C \beta / C \gamma$ regions of the K19 (black), P2R2 (red) and P2R3 spectra. Signals arising in this region in the spectra of P2R2 (red) and P2R3 (blue) suggest that residues in $\mathrm{P} 2$ are rigidified upon fibrillization of

Given the presence of the R3 domain in both P2R3 and K19, the PDSD spectrum of K19 fibrils was superimposed onto that of P2R3. Comparison of the PDSD spectra of P2R3 fibrils and K19 fibrils demonstrated that many cross peaks superimposed. Based on this superposition, a number of P2R3 resonances were tentatively assigned (Figure 3.14a). This included the only aromatic residue, Y310. In addition, the cross peak of T319 overlaid with a cross peak in the threonine $C \alpha / C \beta$ region of $P 2 R 3$ fibrils, which was not observed in P2R2 fibrils (Figure 3.13 and 3.14b). This further supports the assignment of the two threonine cross peaks, which are observed in P2R2 fibrils (Figure 3.13), to the P2 domain.

In the PDSD spectrum of P2R2, a few cross peaks of the R2 domain were tentatively assigned using a recently reported resonance assignment of htau40 fibrils (Figure $3.14 b)^{186}$. The comparison suggested that the $\mathrm{R} 2$ domain is part of the rigid cross- $\beta$ structure in P2R2 fibrils, i.e. it is able to be part of the rigid core of amyloid fibrils. Such a similar observation is reported in the recent cryo-EM structure of heparin-induced tau 
fibrils ${ }^{97}$. On the contrary, in the cryo-EM structureof the amyloid fibrils purified from the brain of a patient with $A D$, the $R 2$ domain was excluded from the rigid core ${ }^{79}$.

The data shown in this section indicate that both P2R2 and P2R3 were able to form fibrils, which could be investigated by ssNMR. The presence of signals in the threonine $\mathrm{C} \alpha / \mathrm{C} \beta$ region of the PDSD spectra, as well as the comparison of these spectra with that of K19, provided evidence for a contribution of the proline-rich region to the rigid cross- $\beta$ structure core of amyloid fibrils.

a

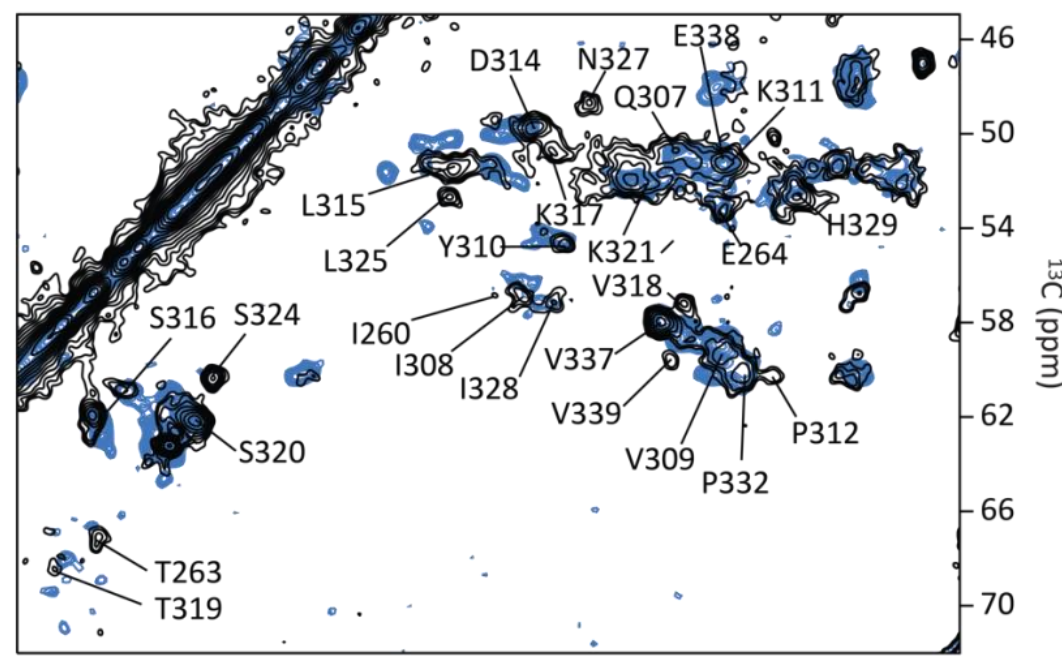

b

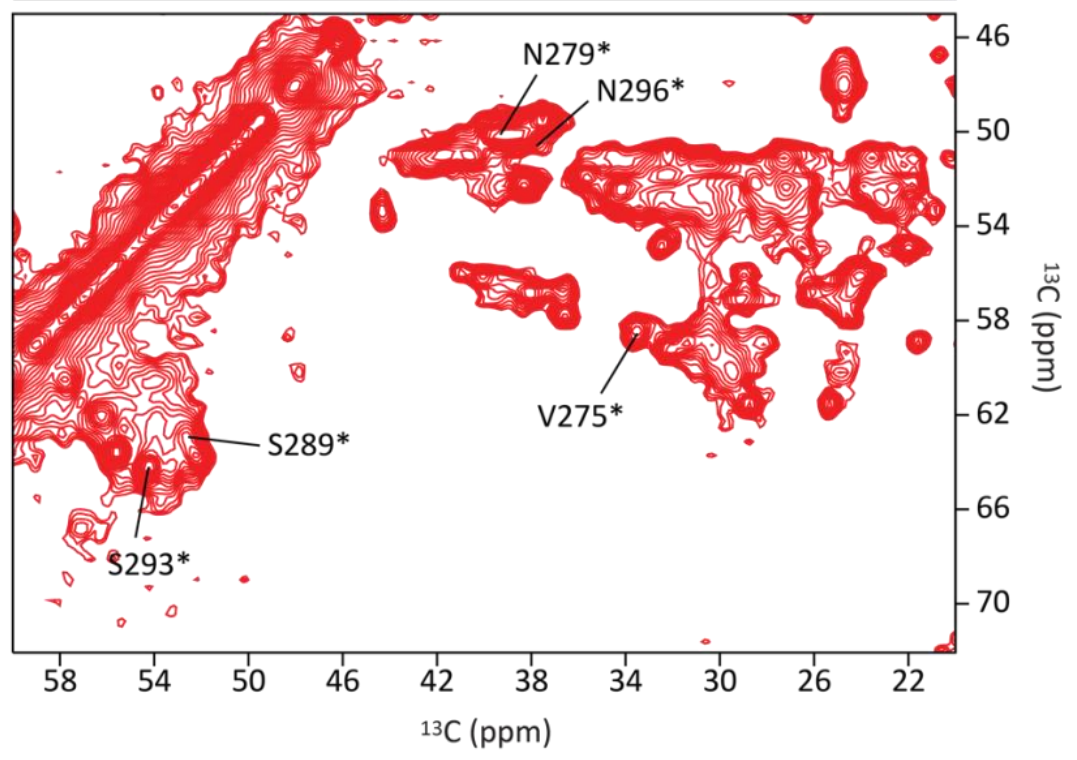

Figure

3.14.

Comparison of the P2R2 and the P2R3 PDSD spectra with a previously reported spectrum of K19 and resonance assignments of htau40 fibrils. a) The assignment of K19 (black) reported by Xiang et al., $2017^{96}$ is shown on top of the PDSD spectrum of K19. The PDSD spectrum of P2R3 (blue) fibrils is superimposed on that of K19. b) Selected resonance assignments of htau40 fibrils (reported by Dregni et al., PNAS 2019186) mapped onto the PDSD spectrum of P2R2

\subsection{Detection of the flexible regions in P2R2 and P2R3 fibrils}


The flexible regions of P2R2 and P2R3 fibrils were probed by measuring $2 \mathrm{D} I N E P T{ }^{1} \mathrm{H}-{ }^{13} \mathrm{C}$ through-bond correlation experiments. In such experiments, residues that are part of the rigid core of amyloid fibrils will not be observed. In the INEPT spectra of both P2R2 and P2R3 fibrils several cross peaks were observed (Figure 3.15 and 3.16). In addition, the INEPT spectra of the two fibrils were similar to each other, suggesting that similar regions remain flexible in P2R2 and P2R3 fibrils.

Using the strategy described above for K32, the INEPT spectra of P2R2 and P2R3 fibrils were compared to the respective ${ }^{1} \mathrm{H}-{ }^{13} \mathrm{C}$ HSQC spectra of the monomeric peptides (Figures 3.15 and 3.16). In contrast to the spectra of $K 32$, the $C \alpha$ and $C \beta$ resonances in the ${ }^{1} \mathrm{H}-{ }^{13} \mathrm{C}$ HSQC spectra of P2R2 and P2R3 fibrils were more dispersed. This simplified their analysis and showed that resonances from proline, glycine and serine residues are present in the 2D INEPT spectra of P2R2 and P2R3 fibrils. Notably, the P2 region contains several proline and serine residues. In contrast, a number of cross peaks were only present in the ${ }^{1} \mathrm{H}-{ }^{13} \mathrm{C}$ HSQC spectra of the monomeric peptides, but were not observed in the ssNMR INEPT spectra. Detailed analysis of the intensities of $\mathrm{C} \alpha$ resonances in the fibrillated $\left(l_{\text {fibrils }}\right)$ and monomeric ( $I_{\text {monomeric }}$ ) spectra identified the regions that remain flexible upon aggregation into amyloid fibrils (Figures 3.17 and 3.18). 


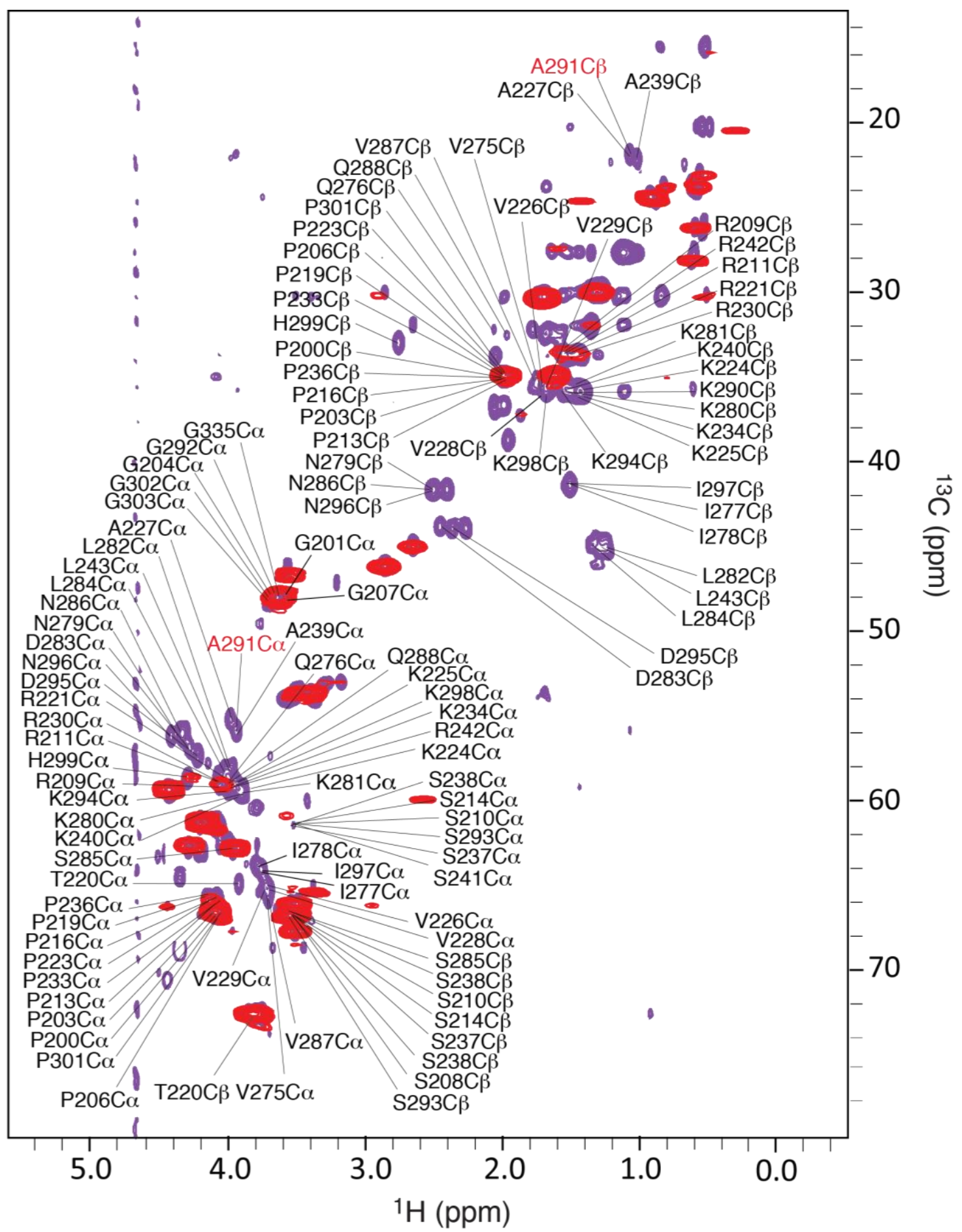

Figure 3.15. Detection of the flexible regions in P2R2 fibrils. a) Superposition of the ${ }^{1} \mathrm{H}-{ }^{13} \mathrm{C} H S Q C$ (purple) and the sSNMR INEPT (red) spectra of monomeric and fibrillized P2R2, respectively. Regions adopting a $\beta$ sheet conformation as a result of the fibrils formation cannot be detected in the INEPT-based SSNMR experiment. The assignments shown refers to the $C \alpha$ and $C \beta$ atoms in the monomeric form of the peptide. The alanine residues in position 291, which is substituting the native cysteine residue, is marked in red. 


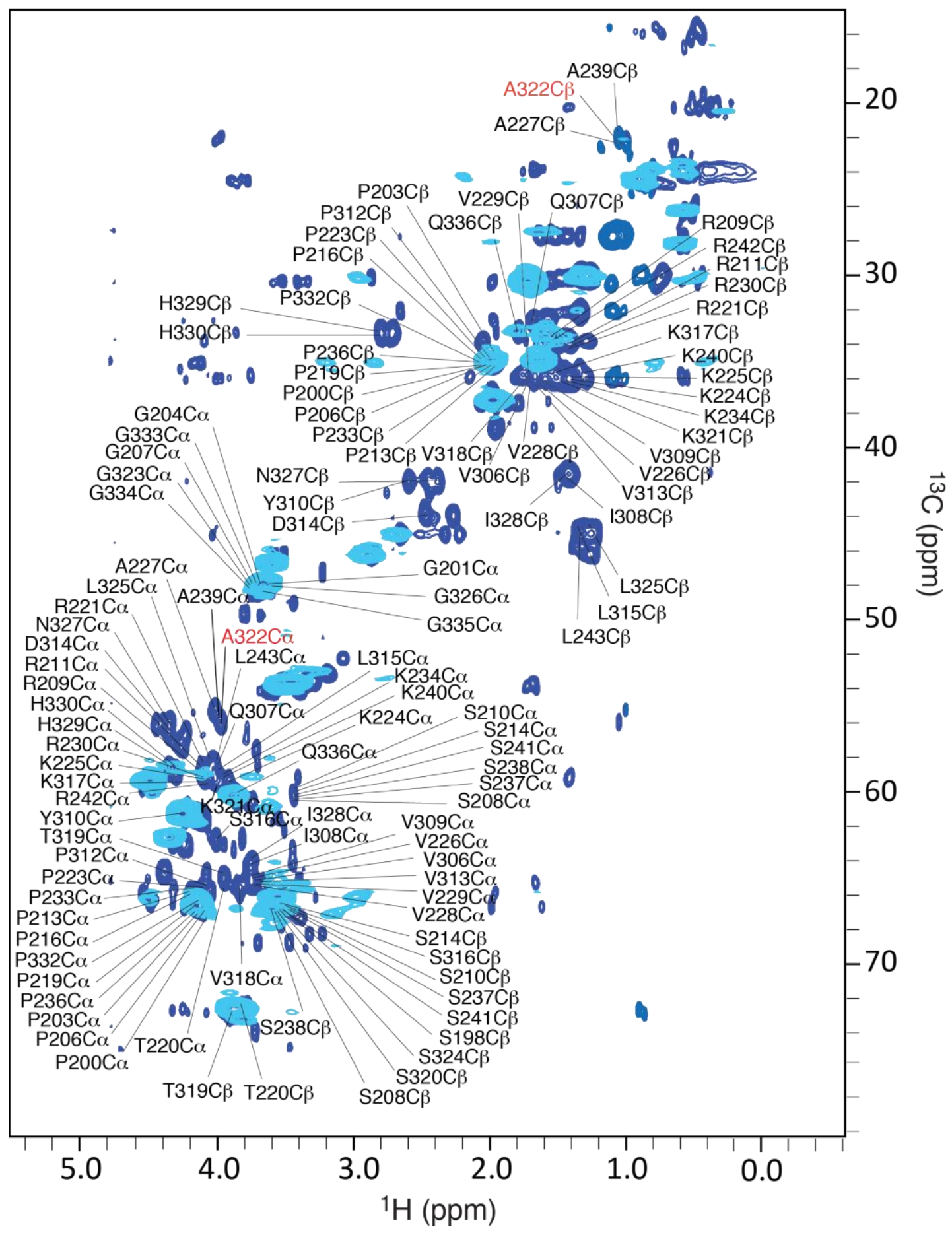

Figure 3.16. Detection of the flexible regions inP2R3 fibrils. a) Superposition of the ${ }^{1} \mathrm{H}-{ }^{13} \mathrm{C} \mathrm{HSQC}$ (light blue) and the sSNMR INEPT (dark blue) spectra of monomeric and fibrillized P2R3, respectively. Regions adopting a $\beta$-sheet conformation as a result of the fibrils formation cannot be detected in the INEPT-based ssNMR experiment. The assignments shown refers to the $C \alpha$ and $C \beta$ atoms in the monomeric form of the peptide. The alanine residues in position 322, which is substituting the native cysteine residue, is marked in red. 
a
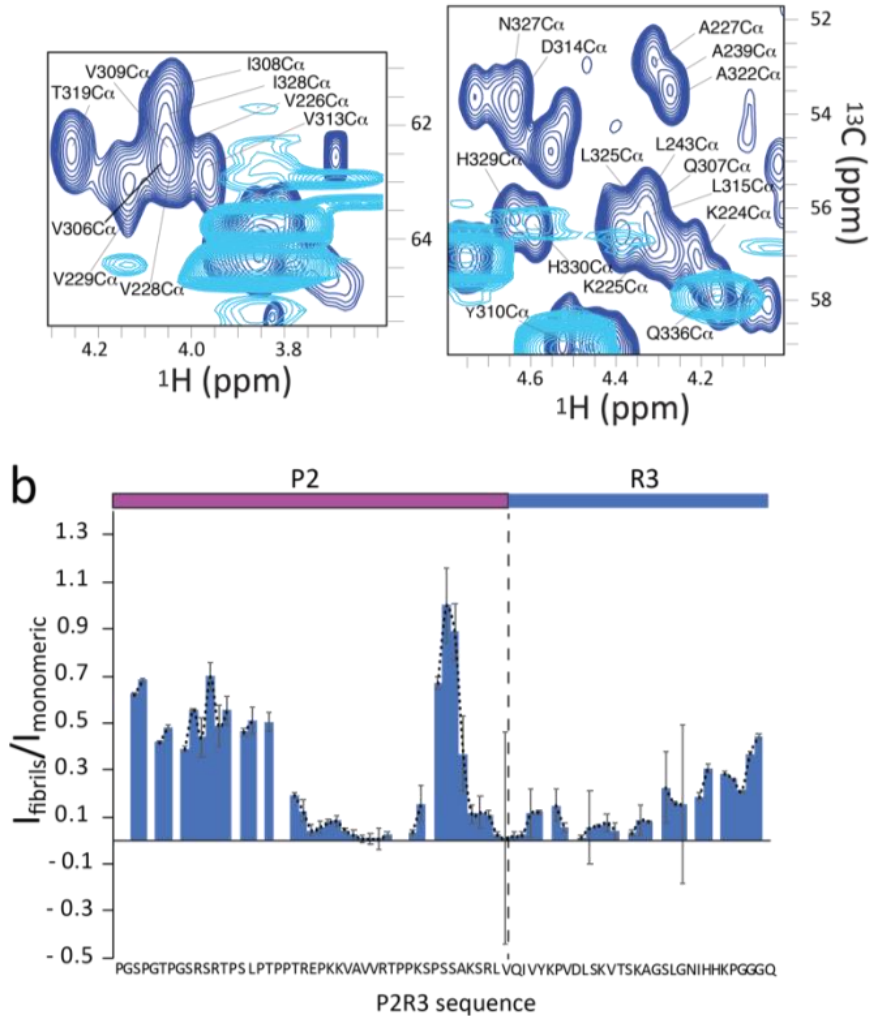

Figure 3.17. Residue-specific mobility in P2R3 fibrils. a) Superposition of the ${ }^{1} \mathrm{H}-{ }^{13} \mathrm{C}$ HSQC (light blue) and the sSNMR INEPT (dark blue) of monomeric and fibrillized P2R3, respectively. Two selected regions of the spectra are shown, corresponding to the regions in the P2 and the R3 domains experiencing loss of NMR signal intensity in the ssNMR INEPT spectrum of the fibrils. b) NMR signal intensity ratios between the fibrillized and the monomeric forms of P2R3. $I_{\text {fibrils }}$ and $I_{\text {monomeric }}$ are cross peak intensities of the fibrils in the SSNMR INEPT spectrum and the monomeric protein in the ${ }^{1} \mathrm{H}-{ }^{13} \mathrm{C}$ HSQC spectrum, respectively. Error bars were calculated on the basis of signal-to-noise ratios in the spectra. The domain organization of P2R3 is represented on top. Asterisks mark residues not assigned in the ${ }^{1} \mathrm{H}^{13} \mathrm{C} \mathrm{HSQC}$. The dashed line shows a threeresidue average.

The residue-specific analysis of P2R3 demonstrated that several residues from the R3 repeat including V306, 1308, V309, D314, N327 and I328 were not observed in the 2D SsNMR INEPT spectrum (Figure 3.17). In addition, K224, V226, A227, V228 and V229, which are part of the ${ }^{225} \mathrm{KVAVVRT}^{231}$ sequence in the $\mathrm{P} 2$ region were broadened beyond detection in the 2D sSNMR INEPT spectrum of P2R3 fibrils (Figure 3.17a-b). In the case of P2R2 (Figure 3.18 ), the cross peaks of the R2 residues 1278, D283 and 1297 were severely attenuated when compared to the HSQC of the monomeric peptide. In addition, the cross peaks of the residues T220, R221, E222 and R230, which are flanking the hydrophobic stretch ${ }^{225} \mathrm{KVAVVRT}^{231}$ in P2, were strongly attenuated (Figure 3.18). The residues V226, A227, 
V228 and V229, located in the ${ }^{225} \mathrm{KVAVVRT}^{231}$ sequence, were completely missing from the 2D sSNMR INEPT spectrum of P2R2 fibrils (Figure 3.18). The analysis further supports a contribution of the P2 domain to the cross- $\beta$ core of amyloid fibrils of P2R2 and P2R3, consistent with the observation of cross peaks in the 2D PDSD spectra of these fibrils that can only be attributed to residues of P2 (Figure 3.14). The linker between the $\beta$-strand in $\mathrm{R} 2$ (or R3) and the rigid residues in P2 (at least residues ${ }^{225} \mathrm{KVAVVRT}^{231}$ ) comprises residues ${ }^{236} \mathrm{PSS}^{238}$ (Figures 3.17 and 3.18). Assuming that all residues that are broadened beyond detection form $\beta$-structure, the analysis suggests the presence of two $\beta$-strands in P2R3 (one in $\mathrm{P} 2$ and one in R3; Figure 3.17). In case of P2R2, an additional short $\beta$-strand might be present at the C-terminus of R3 $\left({ }^{296} \mathrm{NIK}^{298}\right)$ (Figure 3.18).

a
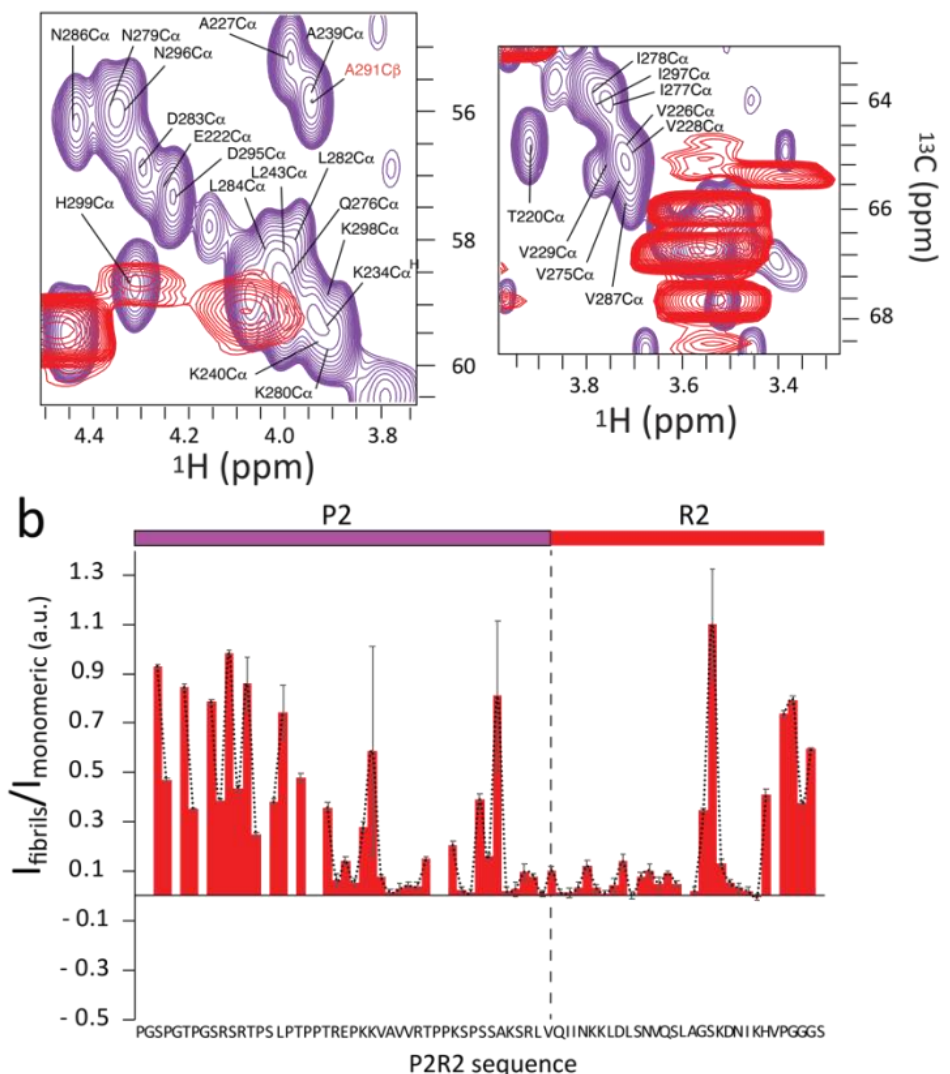

Figure 3.18. Residue-specific mobility inP2R2 fibrils. a) Superposition of the ${ }^{1} \mathrm{H}-{ }^{13} \mathrm{C} H S Q C$ (purple) and the SSNMR INEPT (red) of monomeric and fibrillized P2R2, respectively. Two selected regions of the spectra are shown, corresponding to the regions in the P2 and the R2 domains experiencing loss of NMR signal intensity in the INEPT spectrum of the fibrils. b) NMR signal intensity ratios between fibrillized and monomeric P2R2. $I_{\text {fibrils }}$ and $I_{\text {monomeric }}$ are cross peak intensities of the fibrilis in the INEPT and the monomeric protein in the ${ }^{1} \mathrm{H}$ ${ }^{13} \mathrm{C}$ HSQC spectra, respectively. Error bars were calculated on the basis of signal-to-noise ratios in the 
spectra. The domain organization of P2R2 is represented on top. Asterisks mark residues not assigned in the ${ }^{1} \mathrm{H}^{13} \mathrm{C}$ HSQC. The dashed line shows a three-residue average.

\subsection{Comparison of the PDSD spectra of P2R2, P2R3 and K32}

The P2R2 and P2R3 polypeptides proved to be useful models to probethe contribution of the $\mathrm{P} 2$ domain to the cross- $\beta$ structure of heparin-induced tau fibrils. Especially, the smaller number of residues facilitated a residue-specificanalysis of the ssNMR spectra. The comparative analysis of the PDSD spectra of K19, P2R2 and P2R3 suggested that the signals arising in the $C \alpha / C \beta$ threonine region of the spectra belong to the P2 domain. Then, the K32 PDSD spectrum was superimposed on the PDSD spectra of P2R2 and P2R3 for a similar comparison. As shown in figure 3.19, the cross peaks, which had been assigned to threonine residues in the P2 domain of P2R2 and P2R3, partially matched with cross peaks in the K32 spectrum. This suggested - although the severe signal broadening in the K32 spectrum made further analysis challenging -that the $\mathrm{P} 2$ domain might also be contributing to the cross- $\beta$ structure of K32 fibrils.

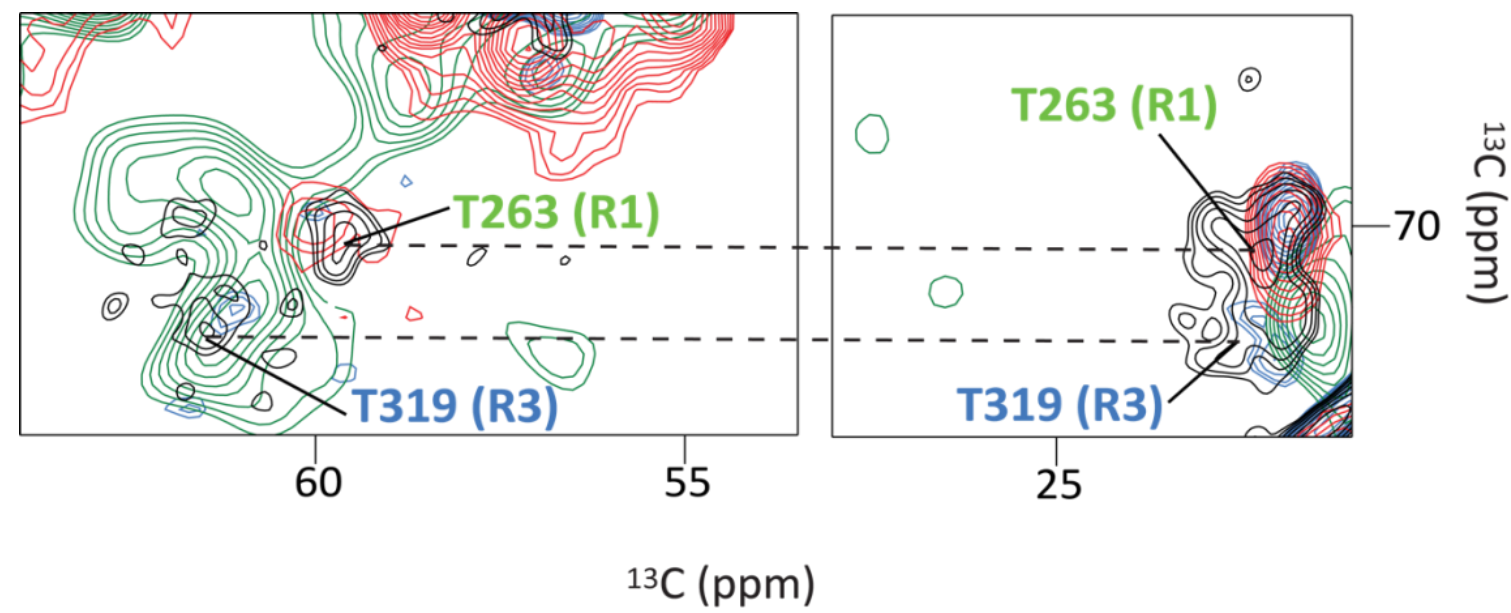

Figure 3.19. Superposition of the threonine region of K19, K32, P2R2 and P2R3 PDSD spectra.The threonine $\mathrm{C} \alpha / C \beta$ and $C \beta / C \gamma$ region of PDSD spectra of K19 (black), K32 (green), P2R2 (red) and P2R3 (blue) is shown. The dashed lines connect the cross-peaks of the $C \alpha / C \beta$ and $c \beta / C \gamma$ region. 


\section{Discussion: project I}

\subsection{The relevance of theproline-rich region of tau in pathological conditions}

The proline-rich region of tau is linked to the protein's role in neurodegenerative diseases ${ }^{36}$. Phosphorylation of the proline-rich region can be detected by several antibodies and is associated with neurodegeneration ${ }^{48,123,125}$. In addition, immunochemistry and X-ray crystallographic studies reported that antibodies present in the blood of healthy individuals recognize epitopes in the P2 domain ${ }^{187,188}$. Despite its importance for tau pathology, however, the proline-region region was not observed in cryo-EM structures of tau fibrils purified from the brains of patients with $A D$ and $P i D^{79,80}$. In addition, the proline-rich region was missing in the cryo-EM structures of amyloid fibrils of htau40 aggregated in vitro in the presence of heparin ${ }^{97}$. The structural role of the proline-rich region in amyloid fibrils of tau is therefore unknown.

\subsection{Structural properties of the rigid core of K32 fibrils}

The use of smaller tau constructs proved to simplify the study of the interaction of tau with microtubules as well as the contribution of different regions to tau aggregation and fibril formation ${ }^{19,50,52}$. For NMR studies, this approach furthermore decreases the signal overlap in the spectra and thus allows a more reliable analysis ${ }^{52}$.

The tau construct K32 was selected, because it contains the P2 domain of the proline-rich region, the four imperfect repeats and the downstream region $R^{\prime}$ of htau $40^{52}$. It thus contains all the parts of tau that were observed in the cryo-EM structures of amyloid fibrils purified from patient brains. Because tau is a highly soluble protein with little tendency to aggregate, heparin and other polyanions are commonly used to induce tau aggregation in vitro. The strong negative charge of these cofactors neutralizes positive charges in tau, thus favoring self assembly ${ }^{51,55,85,86}$. Indeed, fibrils of K32 were obtained upon addition of heparin and subsequently characterized using different biophysical approaches, e.g. ThT fluorescence, CD and electron microscopy (Figure 3.2). Quantification 
of the secondary structure content ${ }^{145}$ detected by $C D$ resulted in the following distribution: $\sim 8 \% \alpha$-helix, $~ 63 \% \beta$-sheet and $28 \%$ turn-like/random-coil conformation (Table 3.1). Because the sequence of $K 32$ comprises 194 residues, the CD analysis suggested that more than 100 residues fold into cross- $\beta$ structure upon aggregation into amyloid fibrils. In the cryo-EM structure of amyloid fibrils of htau40 aggregated in the presence of heparin, i.e. in similar conditions as used here for $\mathrm{K} 32$, residues from the $\mathrm{C}$-terminus of $\mathrm{R} 1$ and all the residues locatedin R2 (31 residues) and R3 (31 residues) folded into rigid cross- $\beta$ structure, thus in total $\sim 65$ residues.

Table 3.1 Secondary structure content calculated with the Dichroweb software ${ }^{189}$ from experimental CD spectra.

\begin{tabular}{lccccc} 
& Helix & Strand & Turn & Unordered & total \\
\hline P2R2 & 0.11 & 0.60 & 0.10 & 0.32 & 0.99 \\
P2R3 & 0.11 & 0.53 & 0.23 & 0.318 & 0.99 \\
K32 & 0.08 & 0.63 & 0.28 & 0.003 & 0.99
\end{tabular}

In order to gain further insight into the structure of K32 fibrils, ssNMR experiments were performed. The superposition of the PDSD spectra of K32 and K19 suggested that residues belonging to the R1 and R3 domains are part of the rigid cross- $\beta$ structure of amyloid fibrils formed by both of these tau constructs in vitro in presence of heparin.

Severe signal overlap in the spectra of K32 precluded a more detailed analysis. At the current stage, it therefore remains unknown if the tau chain folds into a similar conformation in amyloid fibrils of K32 and K19. Because K19 lacks repeat R2 and thus can be regarded as a model for amyloid fibrils formed by three-repeat isoforms of tau, it indeed is more likely that $\mathrm{K} 19$ and K32, which contains four imperfect repeats display a different amyloid fold. Consistent with this hypothesis, the cryo-EM structures of amyloid fibrils purified from patients with $A D$, in which both three-repeat and four-repeat isoforms are detected in insoluble protein deposits ${ }^{79}$, differ from the cryo-EM structure of amyloid 
fibrils purified from $\mathrm{PiD}^{80}$ i.e. a disease that is characterized by the pathological aggregation of three-repeat isoforms of tau ${ }^{190}$. A further factor influencing the structural properties of tau fibrils, is the oxidation of tau's two native cysteine residues C291 and C322. C291 and C322 are located in the R2 and R3 domain, respectively, and can form inter- and intra-molecular disulfide bridges in oxidizing conditions ${ }^{93}$. In the K19 construct used in the current study, the single cysteine residue of K19 was substituted by alanine (C322A). Substitution of cysteine residues to alanine were previously shown to improve the quality of the ssNMR spectra of tau fibrils ${ }^{84,86}$. In the 2D PDSD spectrum of K19, the cross peak of C322A is well isolated from the rest of the resonances (Figure 3.4). In the sequence of $\mathrm{K} 32$, the two native cysteines were maintained and might have contributed to more structural heterogeneity and thus a decreased resolution of its PDSD spectrum.

\subsection{The flexible regions in $\mathrm{K} 32$ fibrils}

A 2D INEPT transfer-based ${ }^{13} \mathrm{C}-{ }^{13} \mathrm{C}$ correlation experiment was used to probe the flexible regions of the fibrils, instead of their rigid core ${ }^{181}$. This approach has been previously applied to K19 fibrils to identify resonances which did not belong to the core of the fibrils ${ }^{84}$. The superposition of the ssNMR INEPT and the assigned ${ }^{1} \mathrm{H}-{ }^{13} \mathrm{C}$ HSQC spectrum of $\mathrm{K} 32$ revealed that proline, glycine and serine residues maintained high flexibility. Residues belonging to the R3 domain, which have been reported to be part of the rigid core of the fibrils ${ }^{79,80}$, the $\mathrm{R} 2$ domain, observed in the core of the heparin-induced fibrils ${ }^{97}$, and to the P2 domain, e.g. V226, A227, V229 and A239, experienced a decrease in their intensity (Figure $3.6 \mathrm{~b}$ and c). Due to the small chemical shift dispersion, many of the amino acids' $\mathrm{C} \alpha / \mathrm{C} \beta$ resonances in the ${ }^{1} \mathrm{H}-{ }^{13} \mathrm{C}$ HSQC of $\mathrm{K} 32$ clustered in the same region, thus the use of this assignment for the identification of the residues in the INEPT spectrum became challenging. On top of that, residual monomeric $\mathrm{K} 32$ in the rotor might have contributed to the signal in the spectrum, making the identification of the residues involved in the fibrils formation difficult. For some few isolated resonances in the $c \beta$ region of the spectrum a complete loss of signal intensity could be observed (Figure 3.6a). The missing peaks belonged to residues located in the R1, the R3 and the R4 domains. The analysis of the PDSD spectrum of $\mathrm{K} 32$ therefore suggested that the R1 and the R3 domain are 
involved in the formation of the rigid core of K32 fibrils. Because of the partial loss of signal intensity observed for the residues in the $\mathrm{P} 2$ domain, a partial rigidification of the $\mathrm{P} 2$ region in amyloid fibrils of $\mathrm{K} 32$ is likely.

\subsection{The use of simplistic models for the study of tau fibrils}

The P2R2 and P2R3 peptides displayed intrinsically disordered properties in solution. The ${ }^{1} \mathrm{H}-{ }^{15} \mathrm{~N}$ HSQC spectra of the peptides showed little chemical shift dispersion (Figure 3.7a and $b$ ). As in the case of full-length htau40, the lack of a distinct secondary structure allows all the amino acids to experience a similar chemical environment ${ }^{56}$. Similar to htau40, the P2R2 and P2R3 peptides retained random coil conformations observed by $C D$ spectroscopy ${ }^{191,192}$ (Figure 3.8c). Their hydrodynamic radii and diffusion rates were determined by diffusion NMR experiments (Figure 3.10). The values obtained (section 3.6) were comparable to previously reported values for unfolded peptides of the same molecular weight ${ }^{171,193}$. Comparison of carbon chemical shifts further showed that residues located in the interior of the $\mathrm{P} 2, \mathrm{R} 2$ and $\mathrm{R} 3$ domains have similar conformational properties in the peptides and in htau40 (Figure 3.9). Together the data show that P2R2 and P2R3 are valid models for the study of tau structure.

\subsection{Biophysical characterization of P2R2/P2R3 fibrils}

The P2R2 and P2R3 peptides formed fibrils upon addition of heparin (Figure 3.11). The P2R3 peptide showed a stronger ThT intensity in comparison to P2R2. The intensity of the ThT fluorescence can be influenced by the conformation of the fibrils with which it interacts $^{185}$. Thus, the difference observed for P2R2 and P2R3 fibrils was suggestive of distinct conformations. Consistent with this, the CD spectra of the fibrils of $P 2 R 2$ and $P 2 R 3$ displayed minima for $\beta$-sheet structure at $\sim 210 \mathrm{~nm}$ and $\sim 220 \mathrm{~nm}$, respectively, and the minimum in the spectrum of P2R2 was less pronounced (Figure 3.11b). The quantification of secondary structure content ${ }^{189}$ resulted in a major contribution from $\beta$-strand ( $60 \%$ ) followed by unordered structure ( $30 \%$ ) and $\alpha$-helical elements ( 10 \%) (Table 3.1). Electron microscopy further revealed different morphologies of P2R2 and P2R3 fibrils 
(Figure 3.11b): P2R2 fibrils were predominantly straight, while P2R3 fibrils displayed a twisted morphology. This suggests that the absence/presence of R2 in three-repeat/fourrepeat tau might influence the morphology of amyloid fibrils in different diseases.

The shorter sequences of P2R2 and P2R3 when compared to K32 or full-length htau40 improved the resolution and decreased the crowding of the ssNMR spectra. Superposition of the ssNMR spectra of P2R3 and K19 further showed good match of many cross peaks, confirming the contribution of the R3 domain in the formation of tau fibrils.

\subsection{The contributionof the $P 2$ domain to the cross- $\beta$ structure of P2R2 and P2R3 fibrils}

The PDSD and INEPT ssNMR spectra of P2R2 fibrils were informativefor two reasons: (i) they showed that the R2 domain isable to form amyloid fibrils, and (ii) the threonine cross peaks observed in the PDSD spectrum demonstrated that part of the P2 domain is contributing to the rigid cross- $\beta$ core of the fibrils. The $R 2$ region is part of the cross $-\beta$ core observed in the cryo-EM structures of heparin-induced htau40 fibrils ${ }^{97}$, but is not present in the cross $-\beta$ core of tau fibrils purified from $A D$ brains ${ }^{101}$. Because both three-repeat and four-repeat isoforms aggregate into insoluble deposits in $A D$, it is currently unclear if the lack of R2 in tau fibrils purified from AD brains is connected to a mixture of different tau isoforms. Notably, an antibody recognizing the hexapeptide in R2 can decreasetau fibrillization, supporting a role of the R2regionin tau aggregation ${ }^{194}$.

Detailed analysis of the INEPT sSNMR spectrum of P2R2 and P2R3 fibrils showed that resonances of residues ${ }^{225} \mathrm{KVAVVRT}^{231}$ of the $\mathrm{P} 2$ domain were strongly attenuated when compared to the monomeric state of the peptides. The ${ }^{225} \mathrm{KVAVVRT}^{231}$ sequence is rich in hydrophobic residues and is homologue to the hexapeptide located in the R2 repeat ${ }^{46}$. The clustal omega ${ }^{195}$ alignment in table 3.2 highlights the similarities of the sequences ${ }^{225} \mathrm{KVAVVRT}^{231},{ }^{275} \mathrm{VIIQNK}^{280},{ }^{306} \mathrm{VQIVYK}^{311}$ : the asterisk indicates the fully conserved valine, while the colon and the dot stand for strong and weak similarities, respectively. Residues marked in red are defined by the program as small and hydrophobic, those in magenta are basic and the amino acids marked in green have a 
hydroxyl group and/or an amine in their side chains. It is clearly visible that the three sequences share a high number of hydrophobic residues, e.g. valine, alanine and/or isoleucine residues. Thus, the establishment of hydrophobic interactions between the ${ }^{225} \mathrm{KVAVVRT}^{231}$ sequence in the P2 domain and the hexapeptides ${ }^{275} \mathrm{VIIQNK}^{280}(\mathrm{R} 2)$ and ${ }^{306}$ VQIVYK $^{311}$ (R3) is likely to be responsible for the recruitment of the proline-rich region into the core of P2R2 and P2R3 fibrils.

Table 3.2 Clustal omega sequence alignment.

\begin{tabular}{l|ccccccc} 
P2(residues 225-231) & K & V & A & V & V & R & T \\
R2(residues 275-280) & K & V & Q & I & I & N & K \\
R3(residues 306-311) & S & V & Q & I & V & Y & K \\
& . & $*$ & & : & : & & .
\end{tabular}

Sequence alignment of the P2, R2 and the R3 domains. The alignment has been performed using the Clustal omega software ${ }^{195}$. Symbol legend: * indicates conserved residues, : indicates high similarity and · indicates weak similarity between amino acids. The colors in the table refer to hydrophobic residues (red), basic amino acids (magenta) and amino acid residues with hydroxyl group and/or an amine (green).

Recently, a sSNMR study on heparin-induced fibrils of the four-repeat tau construct ON4R was published ${ }^{186}$. The assignment reported in this publication was used to identify some of the resonances in the PDSD spectrum of P2R2 fibrils. The authors reported that the R2 and the R3 domains form the rigid core of the fibrils, while the R1 and R4 domains are semi-rigid. In addition, the proline-rich region showed fast anisotropic motions and was defined as semi-mobile by the authors. This supports the evidence reported in this work, where the $\mathrm{P} 2$ domain shows partial mobility and the ${ }^{225} \mathrm{KVAVVRT}^{231}$ sequence is contributing to the rigid fibril core.

At the same time, the data suggest that the semi-mobility of the proline-rich region might be necessary for the regulation of processes at the beginning or right after the aggregation of tau. It might be for example involved in the recruitment of other tau moieties during the aggregation process. Notably, the proline-rich region is an important site of post-translational modifications, i.e. phosphorylation on serine and threonine residues $^{110}$, and is recognized by an anti-tau antibody in the fibrils ${ }^{188}$. Therefore, the partial flexibility and solvent exposition of this region might be involved in aberrant 
phosphorylation events enhancing tau aggregation. A particular attention is given to the T231 comprised in the ${ }^{225} \mathrm{KVAVVRT}^{231}$ sequence, which has been found to be phosphorylated in the CSF of AD patients ${ }^{113,114}$. Therefore, further investigations on the structural properties of the proline-rich region within tau amyloid fibrils and its regulation by phosphorylation might provide insights into the molecular mechanisms of tau aggregation and disease progression. 


\section{Results:project II}

\subsection{Tau interaction with microtubules at different concentrations and temperatures}

Previous NMR studies highlighted four localized regions showing strong signal broadening: $\quad{ }^{225} \mathrm{KVAVVRT}^{231}, \quad{ }^{245} \mathrm{TAPVPMPDL}^{253}, \quad{ }^{275} \mathrm{VQIINKKLDLSNV}^{287}$ ${ }^{306}$ VQIVYKPVDLSKV ${ }^{318}$ 56,98. Additionally, significant signal broadening and chemical shift perturbations were reported for the $\mathrm{P} 1$ and the $\mathrm{R}^{\prime}$ domains ${ }^{56,98} .2 \mathrm{D}{ }^{1} \mathrm{H}-{ }^{15} \mathrm{~N} H S Q C$ spectra of ${ }^{15} \mathrm{~N}$-labeled full-length htau40 were recorded in the presence of paclitaxel-stabilized microtubules. Measurements at $5{ }^{\circ} \mathrm{C}$ and at htau40 and microtubule concentrations of 10 $\mu \mathrm{M}$ and $5 \mu \mathrm{M}$, respectively, showed signal broadening induced in the central domain of htau40 (Figure 5.1a). The P2 domain of the proline-rich region and the pseudo-repeats showed the strongest broadening, in agreement with previously reported NMR data ${ }^{56,98}$.

To gain insight into the temperature dependence of this interaction, $2 \mathrm{D}{ }^{1} \mathrm{H}-{ }^{15} \mathrm{~N}$ HSQC spectra of ${ }^{15} \mathrm{~N}$-labeled htau40 were recorded in presence of paclitaxel-stabilized microtubules at $5{ }^{\circ} \mathrm{C}, 25{ }^{\circ} \mathrm{C}$ and $37{ }^{\circ} \mathrm{C}$. The htau40 concentration used for these experiments was increased to $50 \mu \mathrm{M}$ while the tau to microtubule ratio was kept at 1:0.5. In figure $5.1 \mathrm{~b}$, the intensity plots obtained by measurement of $2 \mathrm{D}{ }^{1} \mathrm{H}-{ }^{15} \mathrm{~N} \mathrm{HSQC}$ spectra at $5{ }^{\circ} \mathrm{C}, 25^{\circ} \mathrm{C}$ and $37{ }^{\circ} \mathrm{C}$ are shown. At $5{ }^{\circ} \mathrm{C}$, the signal broadening started from the $\mathrm{N}$ terminus, where $60 \%$ of the signal intensity was lost: a local minimum in signal intensity could be observed in the region comprising residues from L114 and T123, right before the start of the P1 domain (Figure 5.1b, grey bar). This region was reported to adopt transient $\alpha$-helical structures with amphipatic characteristics in the unbound state ${ }^{56}$. From the $\mathrm{N}$ terminus, the signal broadening gradually strengthened towards the proline-rich region and the pseudo-repeats region, where the intensity decreased below $40 \%$ of the value in the absence of microtubules. Signal intensity was gradually recovered towards the Cterminal region of the sequence (Figure 5.1b). Similar binding profiles were observed at $25^{\circ} \mathrm{C}$ and $37^{\circ} \mathrm{C}$. The differences in the profiles, which were observed at different temperatures, can be due to the temperature-dependence of hydrophobic interactions 
and changes in the exchange rate between the unbound and MT-bound state of tau residues.

a

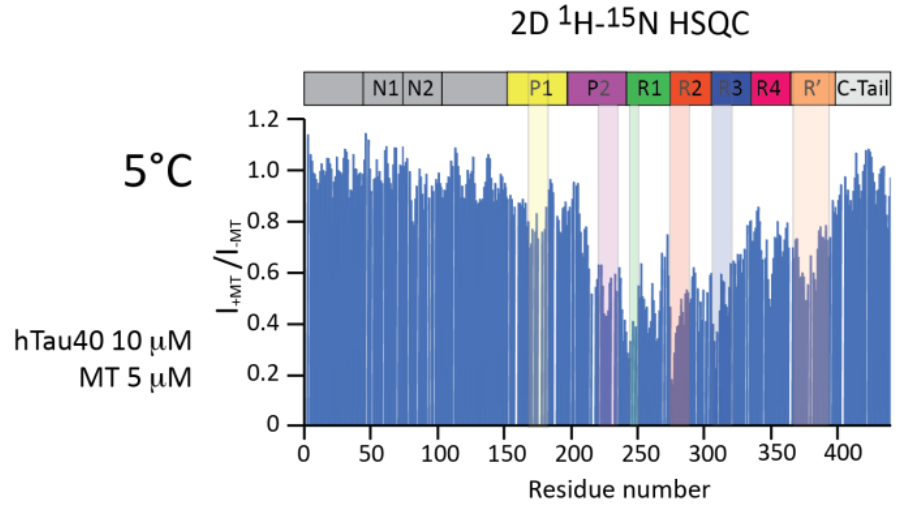

b

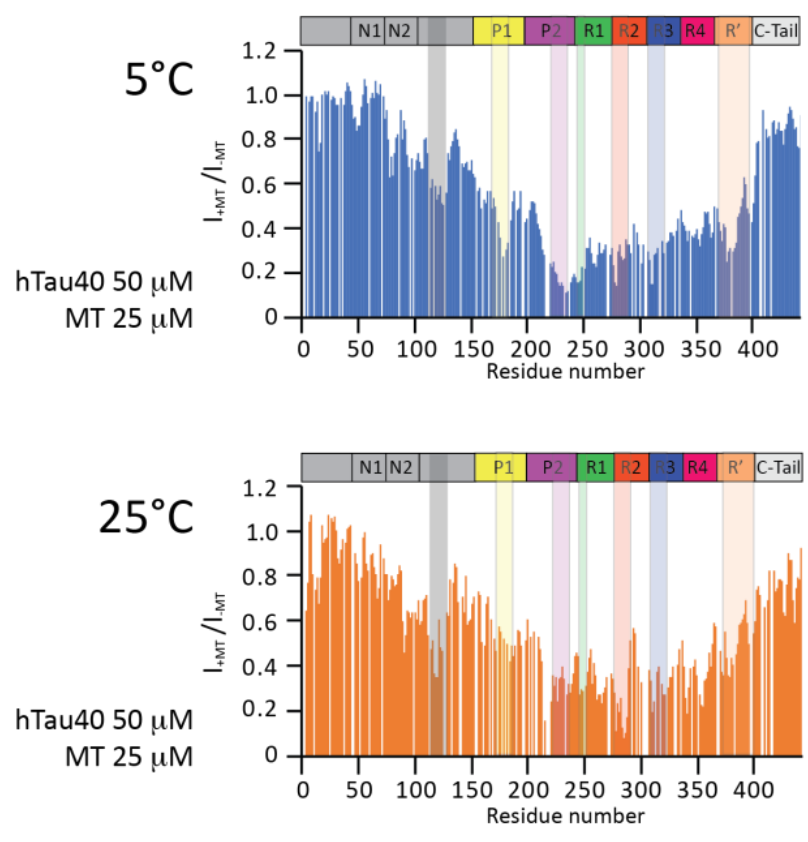

$37^{\circ} \mathrm{C}$

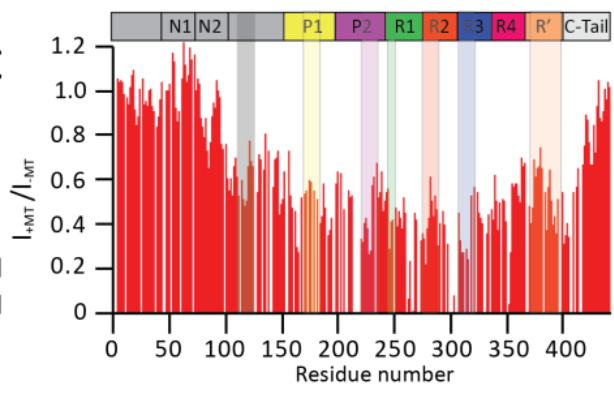

C

$2 D^{1} H_{-15}{ }^{15}$ TROSY

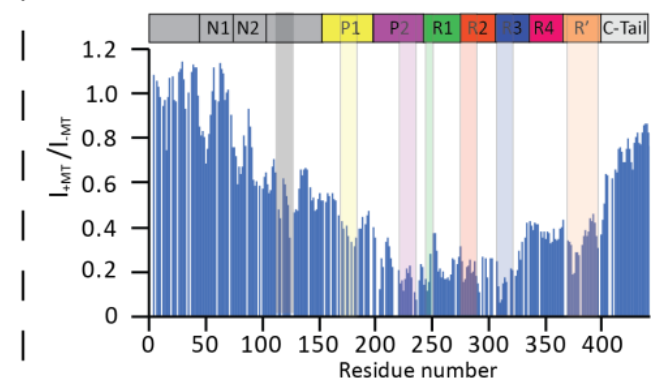

।

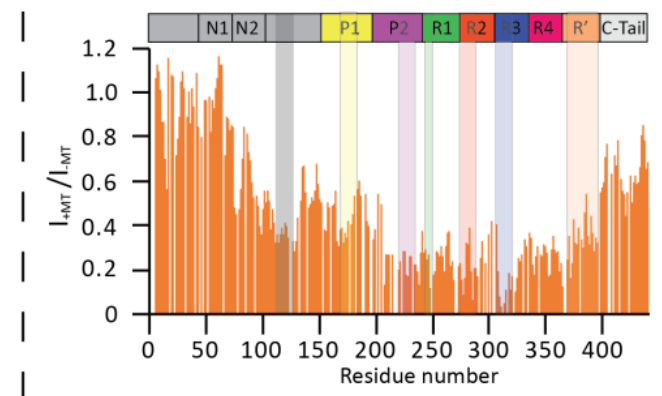

।

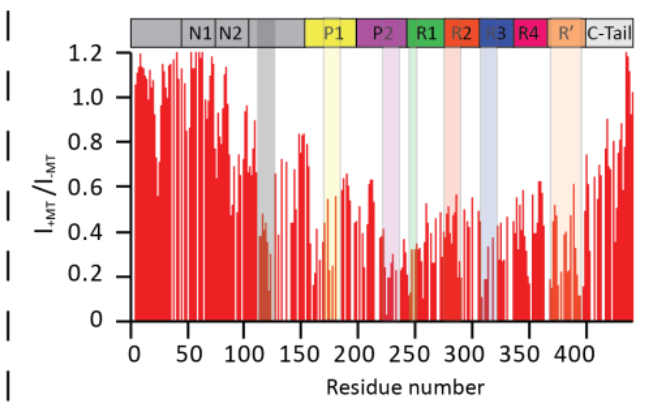

Figure 5.1. NMR profile of tau interacting with paclitaxel-stabilized microtubules at different concentrations and temperatures. a) NMR intensity ratio of htau40 signals in the presence and absence of MTs measured at $5{ }^{\circ} \mathrm{C}$; htau40 concentration of $10 \mu \mathrm{M}$, microtubules $5 \mu \mathrm{M}$ (1:0.5 molar ratio). The binding hot spots reported in previous NMR studies ${ }^{56,98}$ are indicated by color-coded boxes. b-c) Temperature 
dependence of $50 \mu \mathrm{M}$ tau binding to paclitaxel-stabilized microtubules (molar ratio 1:0.5) followed using $2 \mathrm{D}{ }^{1} \mathrm{H}-{ }^{15} \mathrm{~N}$ HSQC (b) and $2 \mathrm{D}{ }^{1} \mathrm{H}-{ }^{15} \mathrm{~N}$ TROSY (c). Temperatures of $5^{\circ} \mathrm{C}, 25^{\circ} \mathrm{C}$ and $37^{\circ} \mathrm{C}$ were used. The region comprising residues L114 to T123, which experiences signal intensity loss, is indicated by the grey bar.

To gain insight into the influence of the transverse relaxation properties of htau40 on the MT-binding profiles, 2D ${ }^{1} \mathrm{H}^{15} \mathrm{~N}$ TROSY experiments were performed at temperatures from $5{ }^{\circ} \mathrm{C}$ to $37^{\circ} \mathrm{C}$ at identical tau/microtubule concentrations (50 $\mu \mathrm{M}$ htau40 and $25 \mu \mathrm{M}$ of microtubules), respectively. Similar to the HSQC experiments, the signal intensity loss started from the $\mathrm{N}$-terminus and expanded towards the middle region of the sequence (Figure 5.1c). At $5^{\circ} \mathrm{C}$ and $25^{\circ} \mathrm{C}$ the $\mathrm{P} 2$, the $\mathrm{R} 1$, the $\mathrm{R} 2$ and the $\mathrm{R} 3$ domains exhibited signal broadening resulting in $40 \%$ of the intensity in the free form. Also in the set of TROSY spectra, the stretch of amino acid residues from L114 to T123 displayed a local minimum in signal intesity (Figure 5.1c).

At $37^{\circ} \mathrm{C}, 60 \%$ of the signal intensity was lost, except for the P2, the R1 and the R3 repeats, whose residues intensities were below $40 \%$ of their values in absence of microtubules. The R2 domain displayed also in this case a loss of $50 \%$ of the intensity. Overall, the HSQC- and TROSY-broadening profiles are similar at the different temperatures, supporting the previously reported location of microtubule-binding hot spots in tau. Notably, microtubule-induced broadening in the P1 region of tau was consistently weaker when compared to the P2 region, suggesting a more pronounced contribution of $\mathrm{P} 2$ of tau to binding to microtubules.

Differences in the binding profile at the same temperature, i.e. $5^{\circ} \mathrm{C}$, but different protein concentration, i.e. $10 \mu \mathrm{M}$ and $50 \mu \mathrm{M}$ emerged, indicated that at higher tau/microtubules concentrations more tau regions are involved in the interaction.

\subsection{Tau interaction with soluble tubulin}

In order to investigate the influence of the polymerization state of tubulin on tau binding, we recorded 2D ${ }^{1} \mathrm{H}-{ }^{15} \mathrm{~N}$ HSQC spectra of $10 \mu \mathrm{M}{ }^{15} \mathrm{~N}$-labeled htau40 in the absence and presence of non-polymerized tubulin heterodimers. The experiments were performed in non-polymerizing conditions, i.e. at $5{ }^{\circ} \mathrm{C}$, and in the absence of GTP. Addition of soluble tubulin caused changes in NMR signal intensities and positions. At a 1:0.5 molar tau-to- 
tubulin ratio, the strongest signal broadening was observed in the $\mathrm{P} 2$, the $\mathrm{R} 1$, the $\mathrm{R} 2$ and the $\mathrm{R} 3$ regions (Figure 5.2). The $\mathrm{P} 1$ and the $\mathrm{R}^{\prime}$ domains also displayed signal loss. At a 1:1 tau to tubulin ratio, the signal intensity in the $\mathrm{P} 1$ domain was below $60 \%$ of its original value, consistent with an increased fraction of tubulin-bound tau. The data shown in this section confirm tau ability to bind both soluble tubulin and microtubule. Tau engages the proline-rich region and the R1, the R2 and the R3 domains of the repeat region to bind to both microtubules and soluble tubulin.

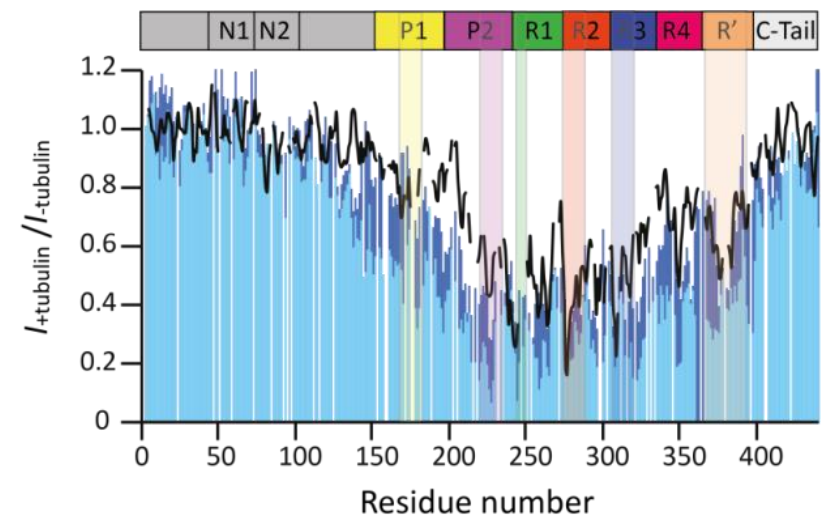

Figure 5.2. NMR profile of htau40 interacting with soluble tubulin. NMR intensity ratio of htau40 signals in the absence and presence of soluble tubulin, measured at $5{ }^{\circ} \mathrm{C}$. The concentration of htau 40 was kept at 10 $\mu \mathrm{M}$, while the tubulin concentration was increased from $5 \mu \mathrm{M}(1: 0.5$ ratio, dark blue) to $10 \mu \mathrm{M}(1: 1$ ratio, light blue). The binding of hTau40 to microtubules at a 1:0.5 molar ratio is shown (black line) for comparison. The binding hot spots suggested in previous NMR studies ${ }^{56,98}$ are indicated by the color-coded boxes.

\subsection{Tau phosphorylation byCdk2/CycA2}

As introduced in section 1.6, phosphorylation is an important post-translational modification, which regulates the cellular activity of tau ${ }^{89,132}$. To gain insight into the effect of phosphorylation on tau interaction with soluble tubulin/microtubules, htau40 was incubated for 24 hours at $30{ }^{\circ} \mathrm{C}$ with catalytic amounts of the proline-directed kinase Cdk2/CycA2 and in presence of ATP (section 2.7). Cdk2/CycA2 phosphorylates serine and threonine residues in the proline-rich region ${ }^{48,130}$. After removal of the kinase, $2 \mathrm{D}{ }^{1} \mathrm{H}-{ }^{15} \mathrm{~N}$ HSQC experiments were recorded on $10 \mu \mathrm{M}$ pTau(Cdk2/CycA2) (Figure 5.3a). Upon phosphorylation, the cross peaks of serine and threonine residues appeared downfield shifted. The cross peaks corresponding to the unmodified residues disappeared or 
partially lost intensity. Based on previously established resonance assignments ${ }^{48,49,56}$, the analysis demonstrated phosphorylation of T153, S202, T205, S210, T220, T231, S235 situated in the proline-rich region and S404 in the R' domain. In addition, the R230 cross peak disappeared from its original position in unmodified tau (Figure 5.3a). In order to further validate this analysis, mass spectrometry was performed on the sample and suggested up to nine phosphorylation sites (Figure 5.3b). On the SDS-PAGE gel, pTau(Cdk2/CycA2) exhibited an increased molecular weight due to the addition of the phosphate groups (Figure 5.3b).

a

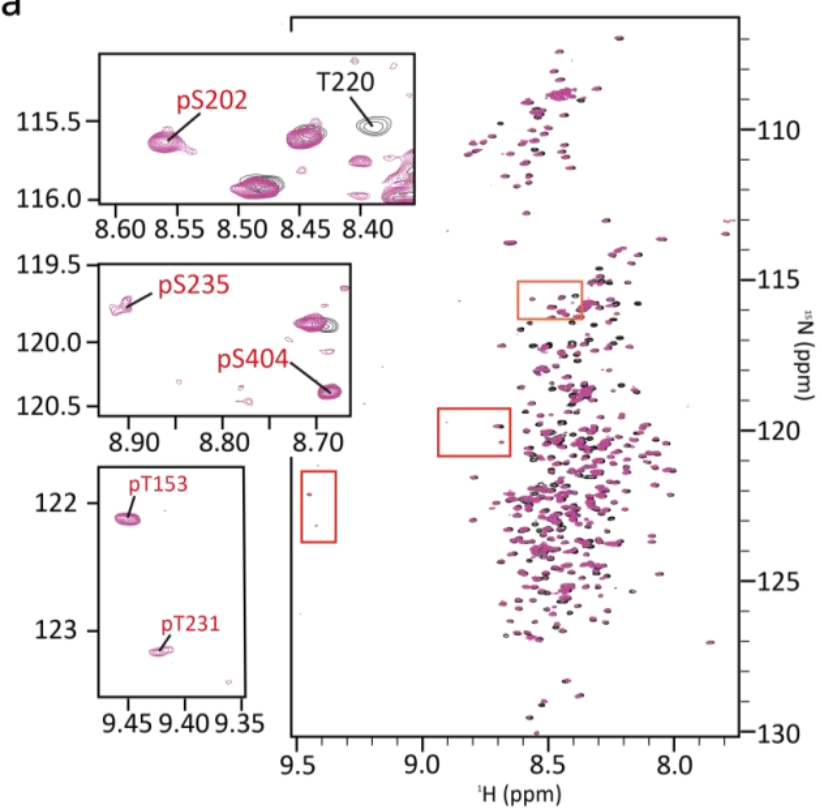

b

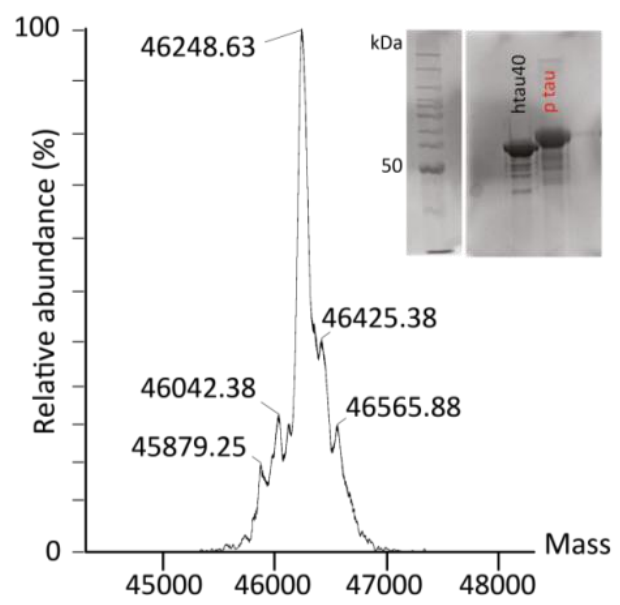

Figure 5.3. Phosphorylation of the proline-rich region of tau by CDK2/CycA2.a) Superposition of ${ }^{1} \mathrm{H}^{-15} \mathrm{~N}$ HSQC spectra of unmodified (black) and phosphorylated htau40 (pink). Targeted serine and threonine residues in the proline-rich region show the typical downfield shift of phosphorylated residues and are shown in the boxes. b) Mass spectrometry of pTau(Cdk2/CycA2) together with SDS PAGE gel analysis. Due to the addition of phosphate group (ca. 80 Da per group), the tau band is shifted upwards.

In order to calculate the degree of phosphorylation in the pTau(Cdk2/CycA2) spectrum, the intensities of the cross peaks for the unphosphorylated and phosphorylated state for each selected residue were determined. For residues S202, T220, S235 and S404, the anaylsis demonstrated nearly complete phosphorylation. For T205 and T220, the degree of phosphorylation was approximately $85-90 \%$, while 60\% phosphorylation was calculated for T231 and T153. Phosphorylated S202 and T205 are recognized by the AD diagnostic antibody AT8, while phosphorylated T231 is detected by the monoclonal 
antibody AT180 ${ }^{196}$. In conclusion, Cdk2/CycA2 phosphorylates the proline-rich region of tau and results in phosphorylated tau that is recognized by AD diagnostic antibodies.

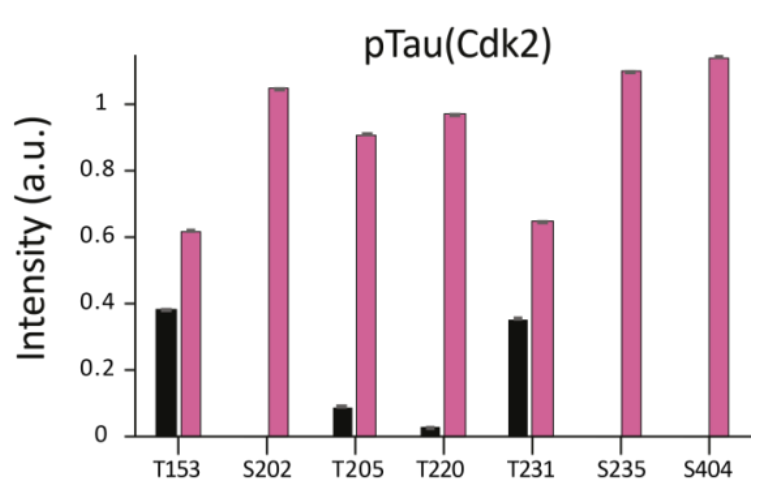

Figure 5.4. Quantification of tau phosphorylation mediated by Cdk2/CycA2. Normalized intensity values of the cross peaks of the unmodified (black bars) and phosphorylated (pink bars) tau residues. Calculation was performed by dividing each cross-peak intensity by the total intensity for any given residue. The total intensity was calculated as the sum of the unmodified and the phosphorylated cross peaks intensities. Error bars where calculated by propagation of the error obtained by the Sparky software.

\subsection{Interaction of phosphorylated tau with tubulin/microtubules}

As shown in the result sections 5.1 and 5.2, tau engages the P2 domain and the pseudorepeat region to interact with both soluble tubulin and microtubules. In order to understand whether phosphorylation in the proline-rich region could interfere with these interactions, 2D ${ }^{1} \mathrm{H}^{15} \mathrm{~N}$ HSQC were recorded on $10 \mu \mathrm{M}$ of pTau(Cdk2/CycA2) in the absence and presence of soluble tubulin heterodimers. Experiments were performed at 5 ${ }^{\circ} \mathrm{C}$ in non-polymerizing conditions. At a pTau(Cdk2/CycA2)-to-tubulin molar ratio of 1:0.5, the strongest signal broadening was observed in the P2 domain and in the pseudo-repeat region (Figure 5.5a-b). Besides, some differences in the P2 and R1 domains could be observed comparing the binding profiles of pTau(Cdk2/CycA2) and unmodified htau40 (Figure 5.5b). The region in which these differences occurred corresponded to the region where the phosphorylated residues cluster (Figure 5.5b). 
a
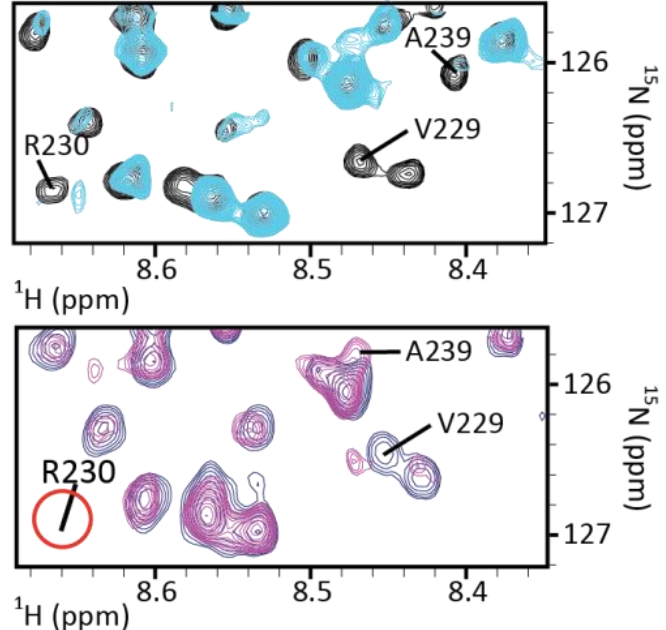

b

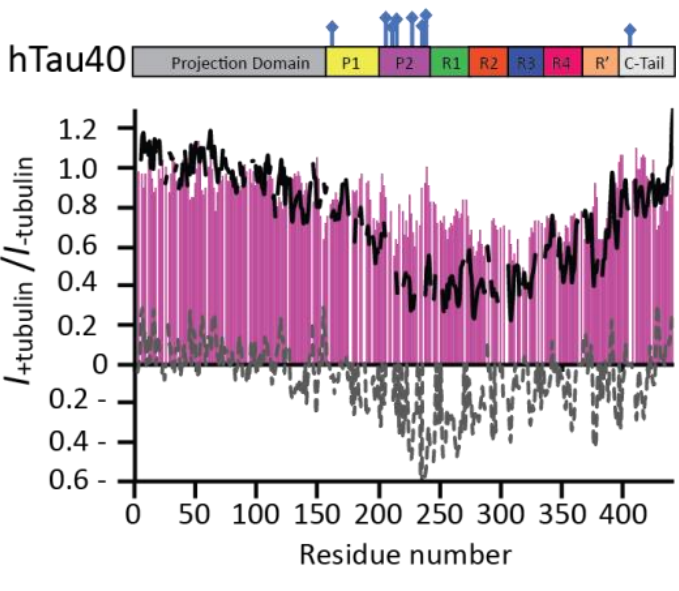

Figure 5.5. Interaction of tau phosphorylated by Cdk2/CycA2 with soluble tubulin. a) Selected region from ${ }^{1} \mathrm{H}-{ }^{-15} \mathrm{~N}$ HSQC spectra of unmodified htau40 in the absence (black) and presence (cyan) of soluble tubulin heterodimers is shown in the upper panel.In the lower panel, a selected region from the ${ }^{1} \mathrm{H}-{ }^{15} \mathrm{~N}$ HSQC spectrum of pTau(Cdk2/CycA2) in its free (pink) and tubulin-interacting state (blue) is shown. Notably, the cross peak of R230 is perturbed upon Cdk2/CycA2phosphorylation. b) Residue-specific tubulin-interaction profile of unmodified hTau40 (black line) and pTau(Cdk2) (pink bars). $I_{\text {-tubulin }}$ and $I_{\text {tubulin }}$ are cross peak intensities in ${ }^{1} \mathrm{H}-{ }^{15} \mathrm{~N}$ HSQC spectra of tau alone and in presence of tubulin, respectively. The grey line shows the difference values between the interaction profile of phosphorylated and unmodified hTau40. On top, the domain organization of tau is shown. Residues phosphorylated in pTau(Cdk2) are marked by blue diamonds.

Similar results were obtained from the analysis of $2 \mathrm{D}{ }^{1} \mathrm{H}-{ }^{15} \mathrm{~N}$ HSQC recorded on $10 \mu \mathrm{M}$ of pTau(Cdk2/CycA2) in the absence and presence of paclitaxel-stabilized microtubules (Figure 5.6a and b). Together the data show that pTau(Cdk2/CycA2) is still able to interact with soluble tubulin and with microtubules. 
a
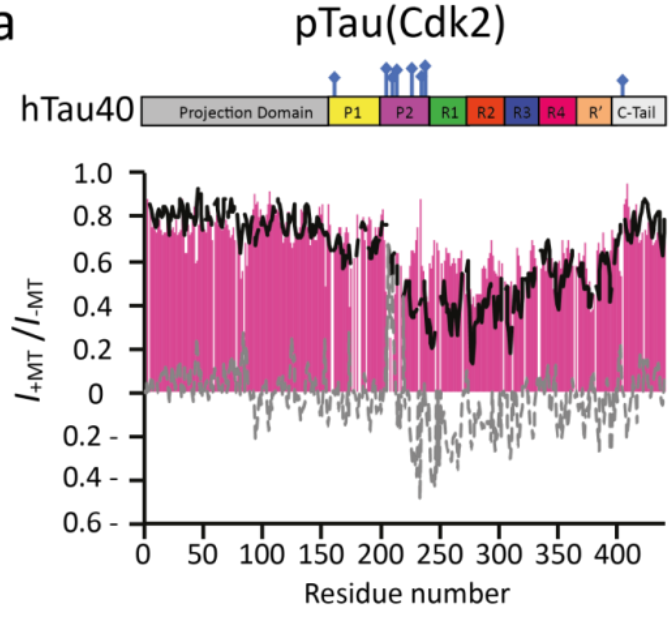

b

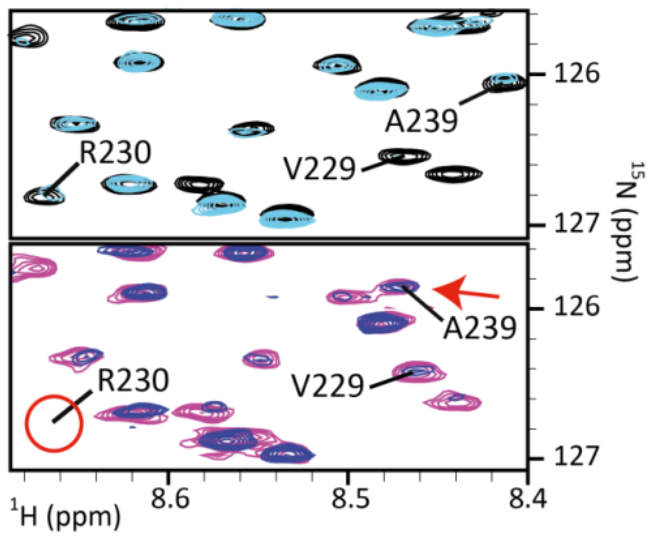

Figure 5.6. Microtubule-interaction of tau phosphorylated by Cdk2/CycA2. a) NMR binding profile of hTau40 (black line) and pTau(Cdk2) (pink bars) in the presence of microtubules. $\mathrm{I}_{-\mathrm{MT}}$ and $\mathrm{I}_{+\mathrm{MT}}$ are cross peak intensities in ${ }^{1} \mathrm{H}-{ }^{15} \mathrm{~N}$ HSQC spectra of tau alone and in presence of microtubules, respectively. The grey line shows the difference values between the interaction profile of phosphorylated and unmodified hTau40. On top, the domain organization of tau is shown. Residues phosphorylated in pTau(Cdk2) are marked by blue diamonds. b) A selected portion of the ${ }^{1} \mathrm{H}_{-}{ }^{15} \mathrm{~N}$ HSQC spectra of htau40 in the free (black) and MT-interacting state (cyan) is shown in the upper panel. Residues disappearing upon interaction are labeled. In the lower panel, a selected portion of the ${ }^{1} \mathrm{H}-{ }^{15} \mathrm{~N}$ HSQC spectrum of pTau(Cdk2/CycA2) in its free (pink) and MTinteracting state (blue) is shown.

\subsection{Tau LLPS and tubulin polymerization}

To gain insight into the contribution of the proline-rich region of tau for tubulin polymerization, an assay based on tubulin polymerization from pre-formed tau droplets was used ${ }^{71}$. Tau droplets were produced by mixing $25 \mu \mathrm{M}$ of htau40 with $10 \%$ dextran in BRB80 buffer (80 mM Pipes, pH 6.8, $1 \mathrm{mM} \mathrm{MgSO}_{4}, 1 \mathrm{mM}$ EDTA, $1 \mathrm{mM}$ DTT). A small concentration of fluorescently labeled htau40 (Alexa 488) was added to the reaction to visualize the presence of tau within the droplets (Figure 5.7a-b). Upon addition of $10 \mu \mathrm{M}$ of tubulin and $1 \mathrm{mM}$ of GTP to the droplets, their immediate deformation could be observed under the microscope(Figure 5.8a). Droplets deformed longitudinally in a bidirectional way and within 30 minutes long microtubule filaments had formed. Red fluorescently labeled tubulin (Alexa 594) was mixed to unlabeled tubulin in order to obtain labeled microtubules. Colocalization of tau on polymerized microtubules was observed by merged red and green fluorescence (Figure 5.8). 

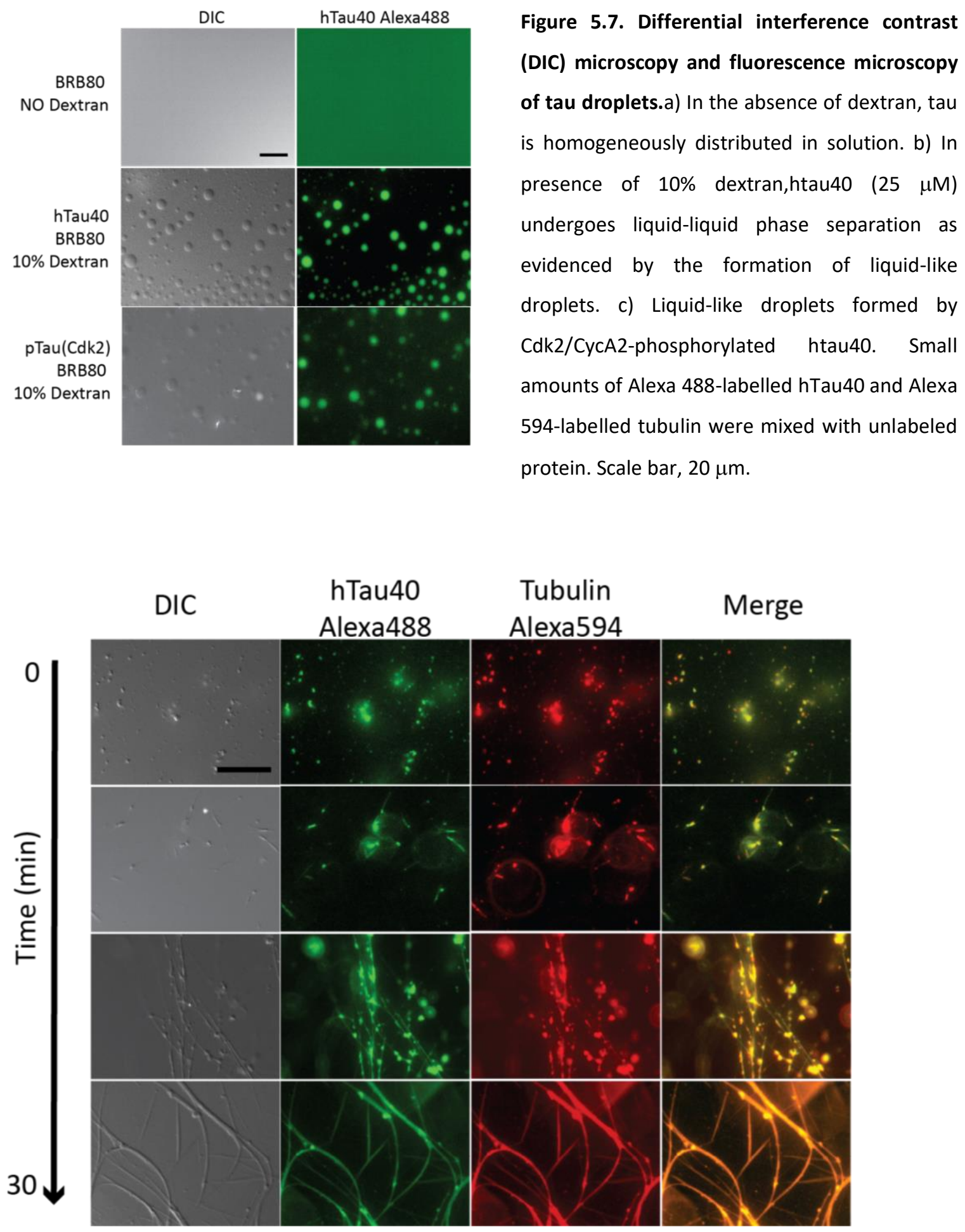

Figure 5.8. Differential interference contrast (DIC) microscopy and fluorescence microscopy of tau droplet-induced tubulin polymerization. Microtubules growth can be observed over time after the addition of tubulin and GTP to htau40 droplets. Small amounts of Alexa 488-labelled htau40 and Alexa 594-labeled tubulin were mixed with unlabeled protein. Superposition of fluorescent htau40 (green) and tubulin (red) images in the merge-column demonstrates colocalization of the two proteins in droplets and for microtubules. Scale bars $20 \mu \mathrm{m}$. 


\subsection{Impact of phosphorylation of tau's proline-rich region on LLPS- mediated tubulin polymerization}

Next, the ability of pTau(Cdk2/CycA2) to undergo LLPS and to polymerize tubulin was addressed. After mixing pTau(Cdk2/CycA2) at room temperature in $10 \%$ dextran, liquidlike droplets were observed by DIC and fluorescent microscopy (Figure 5.7c). This indicated that the phosphorylation did not inhibit tau phase separation. After the addition of tubulin and GTP, fluorescently labeled tubulin was recruited into the droplets but no polymerization of tubulin was observed (Figure 5.9). This showed that phosphorylation of tau in the proline-rich region does neither interfere with tau phase separation in crowded conditions nor recruitment of tubulin to tau condensates, but inhibits tubulin polymerization within the condensed tau phase.

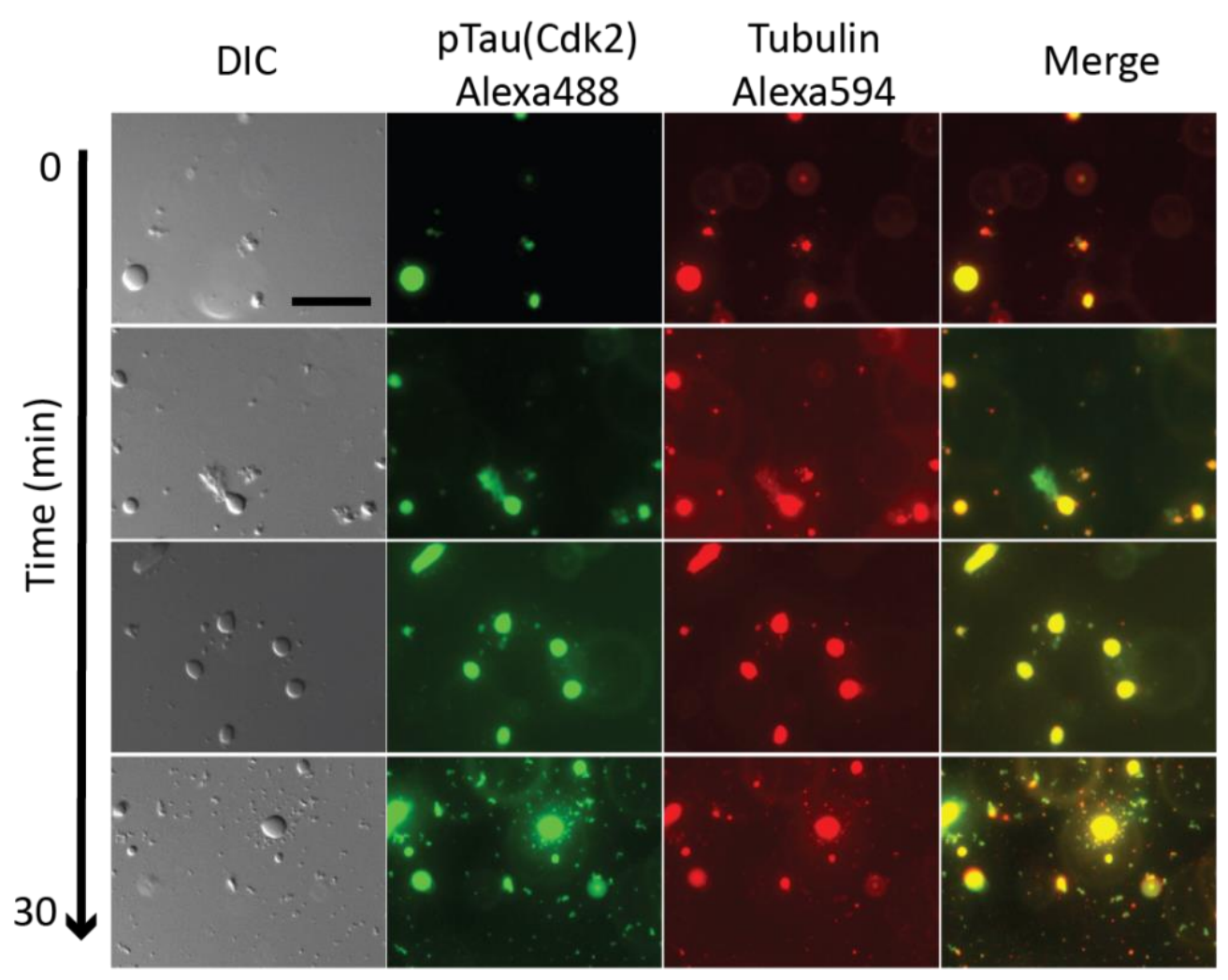

Figure 5.9 Differential interference contrast (DIC) microscopy and fluorescence microscopy of pTau (Cdk2/CycA2) droplets upon addition of tubulin. Upon addition of tubulin and GTP to pTau(Cdk2/CycA2) droplets, polymerization into microtubules was inhibited although tubulin was still recruited into the droplets. Small amounts of Alexa 488-labelled pTau(Cdk2/CycA2) and Alexa 594-labeled tubulin were mixed 
with unlabeled protein. Superposition of fluorescent pTau(Cdk2/CycA2) (green) and tubulin (red) images in the merge-column demonstrates colocalization of the two proteins in droplets. Scale bars, $20 \mu \mathrm{m}$.

\subsection{Tau phosphorylation by MARK2 and interaction with microtubules}

To better interpret the results so far described, phosphorylation of tau in a region different from the proline-rich region was performed. To this extent, htau40 was incubated over night with catalytic amounts of MARK2 and in presence of ATP. MARK2 phosphorylates serine residues in the KGXS motif, conserved in each pseudo-repeat, and attenuates the binding of tau to microtubules ${ }^{90,118}$ (see section 1.6). After removal of the kinase, $2 \mathrm{D}{ }^{1} \mathrm{H}-{ }^{15} \mathrm{~N}$ HSQC experiments were performed on $10 \mu \mathrm{M}$ tau phosphorylated by MARK2 (pTau(MARK2)). New cross peaks appeared downfield shifted in the spectrum of pTau(MARK2) (Figure 5.10a). Their identification was possible using the previously reported resonance assignment ${ }^{118}$. From the analysis, the cross peaks of phosphorylated S262, S305, S324, S356 and S416 were identified (Figure 5.10a, zoomed in box). The degree of phosphorylation was calculated as for pTau(Cdk2/CycA2). Residual intensity for the cross peaks in the unphosphorylated state suggested that the phosphorylation reaction was incomplete. As shown in the histogram plot of Figure 5.11, S356 yielded the highest degree of phosphorylation ( 80\%), S262 and S324 were phosphorylated 50\% and $\sim 60 \%$, respectively. S302 and S416 exhibited the lowest degree of phosphorylation, below $20 \%$. Besides, mass spectrometry suggested up to 10 phosphorylation sites (Figure 5.10c), consistent with previous observations ${ }^{118}$. Other serine residues, e.g. S293, S352 and S413 for which no unambiguous assignment could be found, might have been phosphorylated as well. Next, 2D ${ }^{1} \mathrm{H}-{ }^{15} \mathrm{~N}$ HSQC spectra were recorded on $10 \mu \mathrm{M}$ of pTau(MARK2) in presence of paclitaxel-stabilized microtubules (1:0.5 molar ratio). The NMR binding profile revealed signal broadening in the P2 domain and the pseudo-repeat region (Figure 5.12), with intensities below $80 \%$ of the values in the free form. When compared to the NMR binding profile of unmodified htau40 (Figure 5.12, black line) the signal broadening in the pseudo-repeats region was attenuated, i.e. the signal intensities in the absence and presence of microtubules were more similar, in particular in proximity to the strongly phosphorylated residues S262, S324 and S356 (Figure 5.12, dashed grey 
line). This observation together with the quantification of the degree of phosphorylation, suggested that although the binding to the microtubules was weakened by the phosphorylation with MARK2, residual unphosphorylated htau40 might have contributed to the interaction with the microtubules. In contrast, signal broadening in the $\mathrm{P} 2$ region remained unaffected by MARK2-phosphorylation, suggesting that the P2 region and the pseudo-repeats bind in a largely independent manner to microtubules (Figure 5.12, dashed grey line).
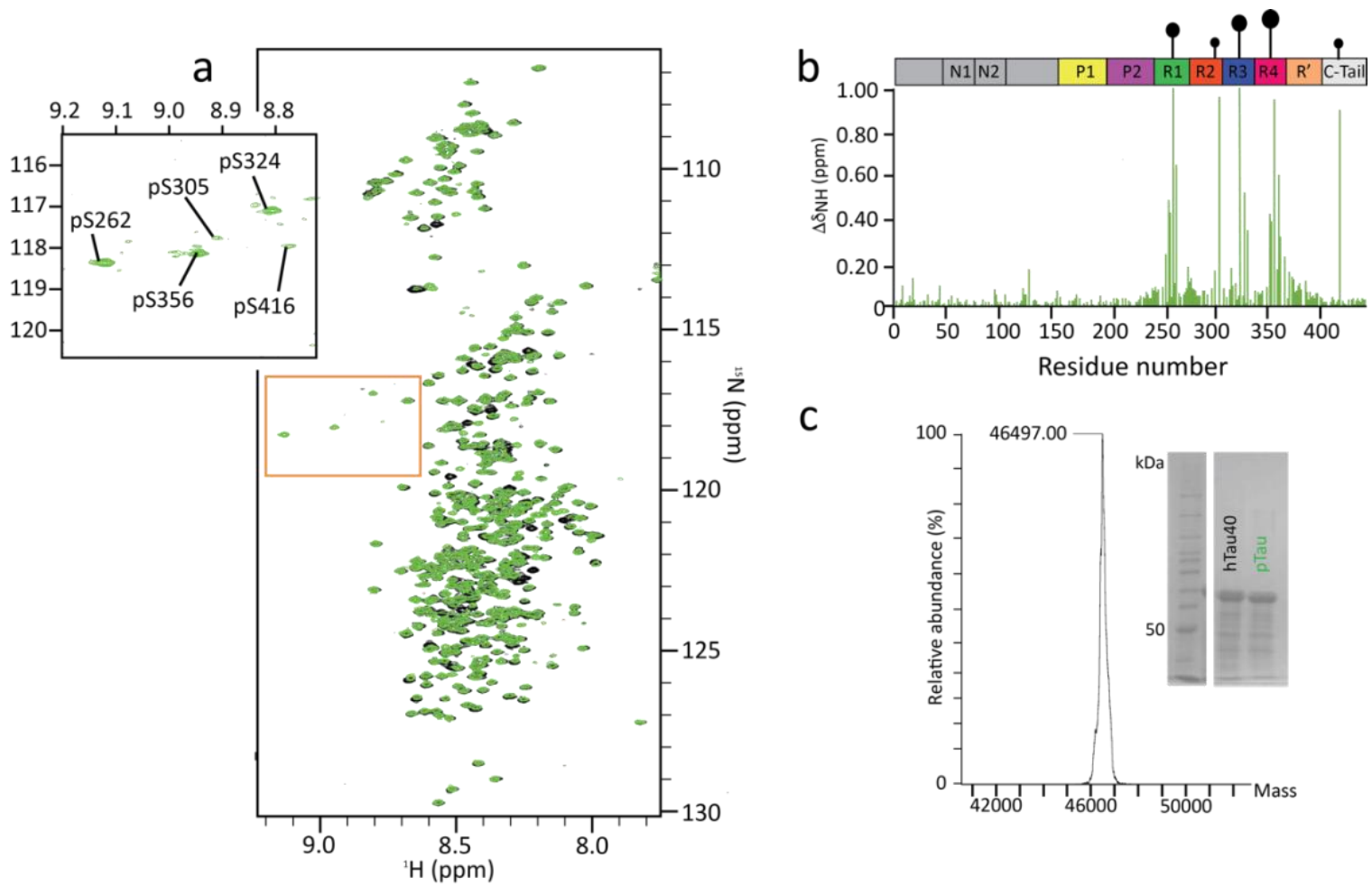

C

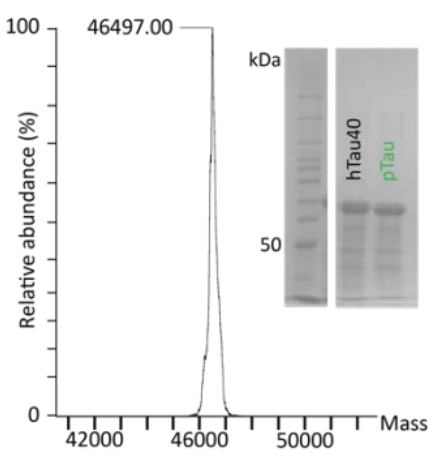

Figure 5.10. Phosphorylation of tau mediated by the kinase MARK2.a) Superposition of ${ }^{1} \mathrm{H}-{ }^{15} \mathrm{~N}$ HSQC spectra of htau40 (black) and pTau(MARK2) (green); phosphorylated serine residues are shown in the box. b) Chemical shift perturbation plot together with tau domain organization. The phosphorylation in the pseudo-repeat region is indicated by black circles. c) Electrospray mass spectrum of the phosphorylated tau indicating one population of 9 to 10 times phosphorylated protein. Next to it, SDS PAGE electrophoresis gel of htau40 (control) and pTau(MARK2). 


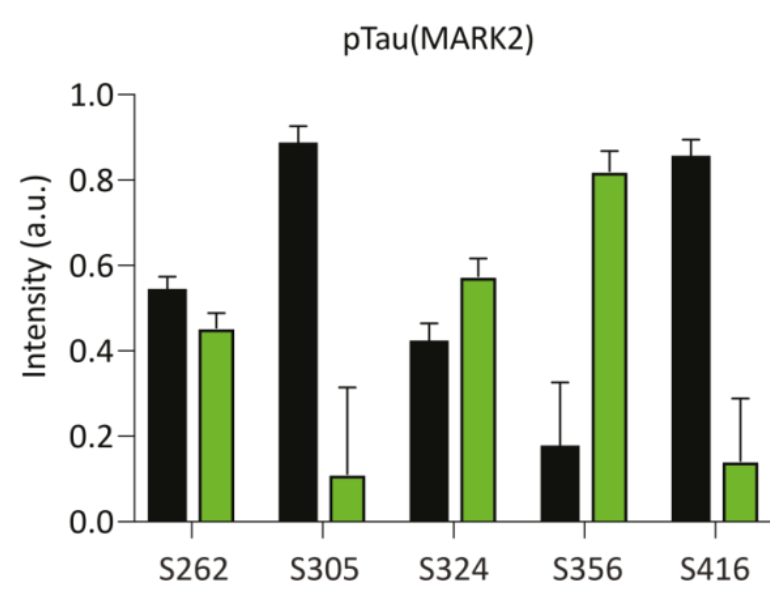

Figure 5.11. Quantification of tau phosphorylation mediated by MARK2. Histogram plot with intensity values for the cross peaks of the unmodified (black bars) and phosphorylated (green bars) selected residues. Calculation was performed by dividing each cross-peak intensity by the total intensity for any given residue. The total intensity was calculated as the sum of the unmodified and the phosphorylated cross peak intensities. Error bars where calculated by propagation of the error obtained from the SPARKY software.

\subsection{Impact of phosphorylation of tau'spseudo-repeat region on}

\section{LLPS-mediated tubulin polymerization.}

The ability of pTau(MARK2) to undergo LLPS and to polymerize tubulin was investigated and compared to that of pTau(Cdk2/CycA2). For this purpose, $25 \mu \mathrm{M}$ of pTau(MARK2) was mixed at room temperature in $10 \%$ dextran. Liquid-like droplets were observed by DIC and fluorescent microscopy (Figure 5.13a), demonstrating that the MARK2phosphorylated protein can phase separate in conditions of molecular crowding. After the addition of tubulin and GTP, microtubules filaments started to appear under the microscope and werevisualizedwith fluorescent microscopy (Figure 5.13b). This suggested that despite the phosphorylated state, pTau(MARK2) could still promote tubulin polymerization. This result further supported the relevance of the proline-rich region in the regulation of tubulin polymerization. 


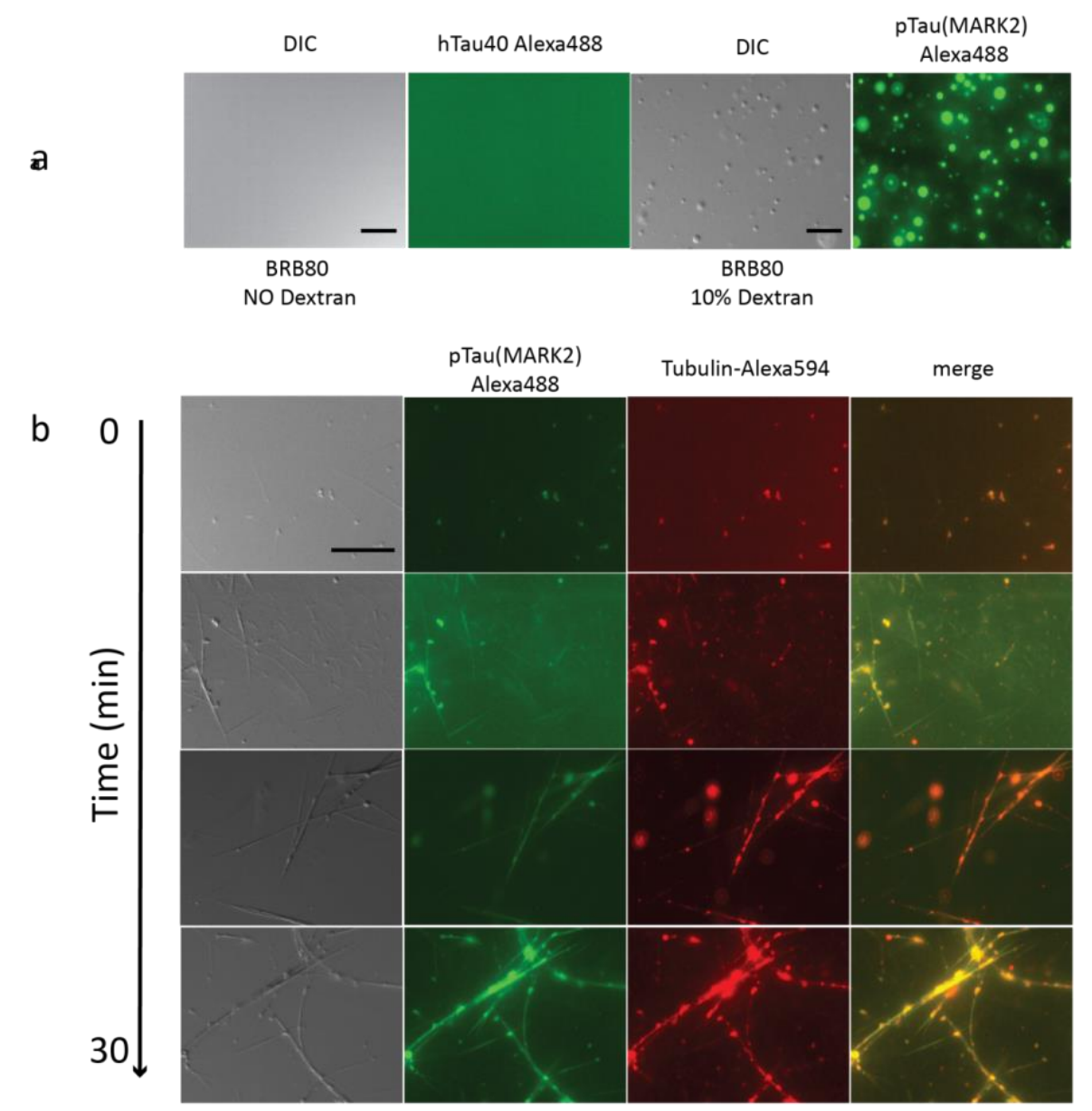

Figure5.13. Differential interference contrast (DIC) microscopy and fluorescence microscopy of pTau(MARK2) droplet-induced tubulin polymerization.a) In absence of dextran tau is homogeneously distributed in solution; in presence of $10 \%$ dextran the protein phase separates at a concentration of 25 $\mu \mathrm{M}$. Upon MARK2 phosphorylation, tau maintains its ability to phase separate. b) Microtubule growth can be observed over time after the addition of tubulin and GTP to pTau(MARK2) droplets. Small amounts of Alexa 488-labeled hTau40 and Alexa 594-labeled tubulin were mixed with unlabeled protein. Superposition of fluorescent pTau(MARK2) (green) and tubulin (red) images in the merge-column demonstrates colocalization of the two proteins in droplets and on microtubules. Scale bars, $20 \mu \mathrm{m}$.

\subsection{Binding of tau'sproline-rich region to soluble tubulin}

As shown so far, the phosphorylation mediated by $\mathrm{Cdk} 2 / \mathrm{CycA} 2$ in the proline-rich region impaired tubulin polymerization from tau droplets, while phosphorylation in the pseudorepeat region mediated by MARK2 did not. To gain further insight into the involvement of the proline-rich region in the process of tubulin polymerization, a peptide corresponding to the P2 region of tau, was prepared by solid-phase synthesis. As shown in figure 5.14, 
the peptide $\operatorname{Tau}(\mathrm{P} 2)$ comprises residues $\mathrm{R} 211-\mathrm{R} 242$ of the $\mathrm{P} 2$ region, including the sequence ${ }^{225} \mathrm{KVAVVRT}^{231}$ involved in the binding to tubulin/microtubules ${ }^{56}$. Saturationtransfer difference (STD) NMR ${ }^{167}$ was used to quantify the interaction strength of Tau(P2) with unpolymerized tubulin. As introduced in section 2.11.7, STD-NMR is a powerful NMR method to study ligand/protein-interactions and depends on the fast off-rate of a ligand from the protein (or high molecular weight binding partner) (Figure 2.5). STD NMR experiments were recorded on $800 \mu \mathrm{M}$ Tau(P2) at different irradiation times, in order to create a build-up curve for the STD signal (Figure 5.14b and c). The magnetization transfer from tubulin to several residues of Tau(P2), including T220, A227, A239 and T231 (Figure $5.14 b)$, indicates that these residues get in direct contact with tubulin residues upon binding of Tau(P2) to tubulin.

a

b

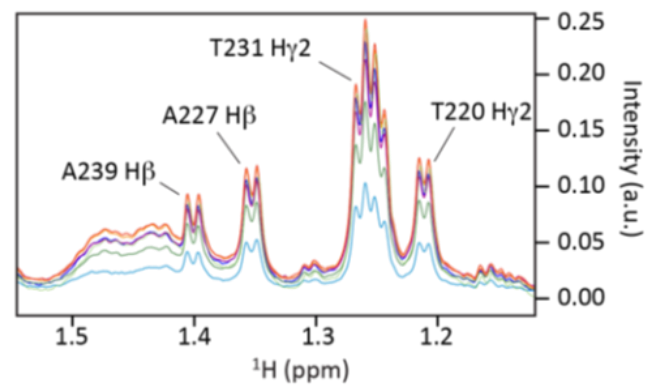

C

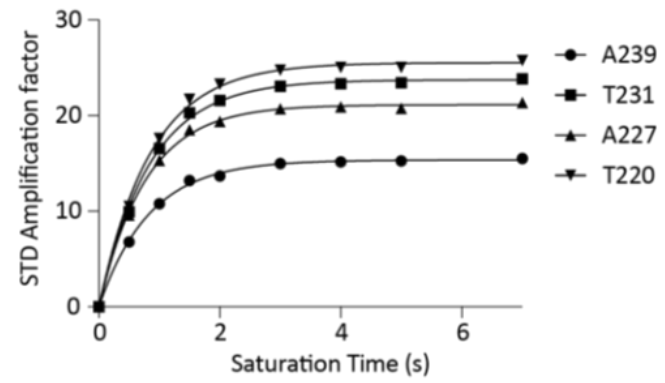

Figure 5.14. STD NMR measurements on Tau(P2). a) Schematic representation of Tau(P2) and its sequence. b) Superposition of ${ }^{1} \mathrm{H}$ 1D STD spectra for different irradiation times, showing the buildup of the STD signal for Tau(P2): $0.5 \mathrm{~s}$ (light blue), $1.0 \mathrm{~s}$ (green), $1.5 \mathrm{~s}$ (blue), $2 \mathrm{~s}$ (purple), $3 \mathrm{~s}$ (light green), $4 \mathrm{~s}$ (orange), $5 \mathrm{~s}$ (yellow) and $7 \mathrm{~s}(\mathrm{red})$. The proton assignment is indicated. c) STD buildup curves obtained by measurement of ${ }^{1} \mathrm{H} 1 \mathrm{D}$ STD spectra at different irradiation times. Measurements were performed at $5{ }^{\circ} \mathrm{C}$ in a non-polymerizing BRB80 buffer ( 80 mM Pipes, pH 6.8, 1 mM MgSO 4,1 mM EDTA, 1 mM DTT). Error bars were calculated according to the spectral signal-to-noise ratio.

Next, the saturation transfer was quantified as a function of Tau(P2) concentration and $\mathrm{K}_{\mathrm{D}}$ values of $\sim 1-2 \mathrm{mM}$ were obtained (Figure 5.15 and Table 5.1). Previous studies showed that isolated, individual pseudo-repeats bind with an affinity of $\sim 200-500 \mu \mathrm{M}$ to tubulin $^{108}$. The affinity of the $\mathrm{P} 2$ region of tau for unpolymerized tubulin is thus a factor of 2-10 lower when compared to the four pseudo-repeats. 
a

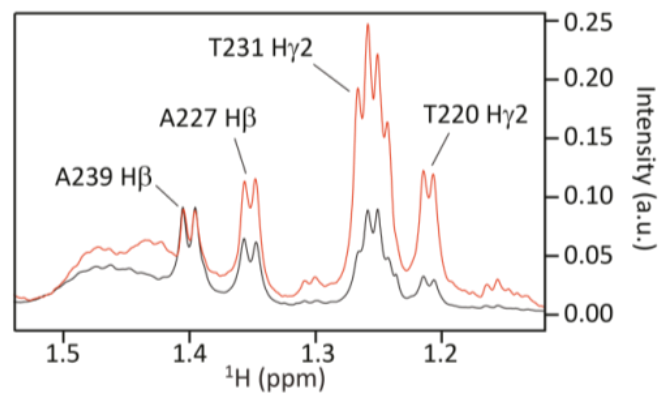

b

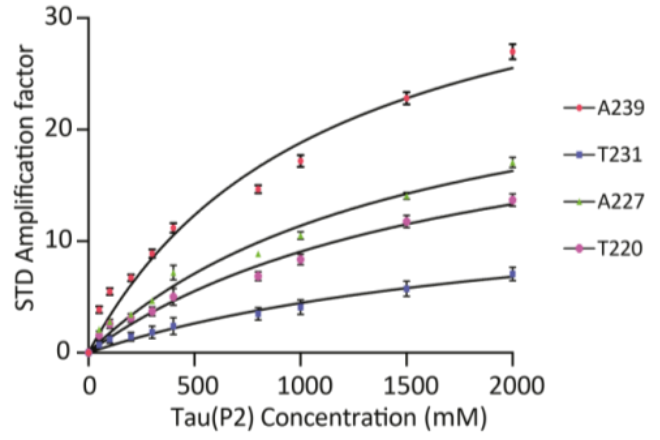

Figure 5.15. Tau(P2) binding affinity to soluble tubulin. a) Superposition of the ${ }^{1} \mathrm{H} 1 \mathrm{D}$ spectrum (red) and ${ }^{1} \mathrm{H}$ 1D STD spectrum (black) of Tau(P2). The proton resonance assignments for the selected residues are indicated. b) Quantification of Tau(P2) saturation transfer as a function of peptide concentration. The $K_{D}$ values were calculated by titrating increasing concentrations of Tau(P2)to a fixed concentration of tubulin $(5 \mu \mathrm{M})$. Measurements were performed at $5^{\circ} \mathrm{C}$ in non-polymerizing BRB80 Buffer ( $80 \mathrm{mM}$ Pipes, pH 6.8 1 $\mathrm{mM} \mathrm{MgSO}_{4}, 1 \mathrm{mM}$ EDTA, $1 \mathrm{mM}$ DTT). Error bars were calculated on the basis of the spectral signal-to-noise ratio.

Table 5.1. $K_{D}$ values for selected residues of the proline-rich region.

\begin{tabular}{|c|c|c|}
\hline Residue & Tau(P2) $K_{\mathrm{D}}(\mathrm{mM})$ & $\mathrm{Tau}(\mathrm{P} 2)-\mathrm{T} 231 \mathrm{p} K_{\mathrm{D}}(\mathrm{mM})$ \\
\hline $\mathrm{T} 220$ & $(1.78 \pm 0.67)$ & $(7.07 \pm 5.07)$ \\
$\mathrm{A} 227$ & $(1.52 \pm 0.40)$ & $(5.22 \pm 1.90)$ \\
$\mathrm{T} 231$ & $(2.25 \pm 0.51)$ & $(13.37 \pm 21.51)$ \\
$\mathrm{A} 239$ & $(1.11 \pm 0.28)$ & $(3.65 \pm 1.62)$ \\
\hline
\end{tabular}

\subsection{Structural insights into the interaction of $\operatorname{Tau}(\mathrm{P} 2)$ with microtubules}

To gain insight into the structural basis of the interaction of the proline-rich region with tubulin and thus the mechanistic basis of LLPS-mediated tubulin polymerization, twodimensional transfer Nuclear Overhauser Effect (trNOE) experiments (see section 2.11.5) were performed. Similar to STD-NMR, the trNOE effect depends on the fast release of a ligand/protein from a high-molecular binding partner, but can additionally provide insight into the structure of the bound ligand ${ }^{162}$. The intensity of trNOE signals strongly depends on the excess of ligand over protein and the molecular weight of the protein, therefore trNOE experiments were performed on Tau(P2) in presence of microtubules. 2D NOESY 
spectra have been measured on $1 \mathrm{mM} \operatorname{Tau}(\mathrm{P} 2)$ in the absence and presence of microtubules. In the absence of microtubules, mostly intra-residual and sequential contacts were present (Figure 5.16a, yellow spectrum). Upon addition of microtubules, new cross peaks appeared due to the formation of medium and long-range NOE contacts (Figure 5.16a, black spectrum).
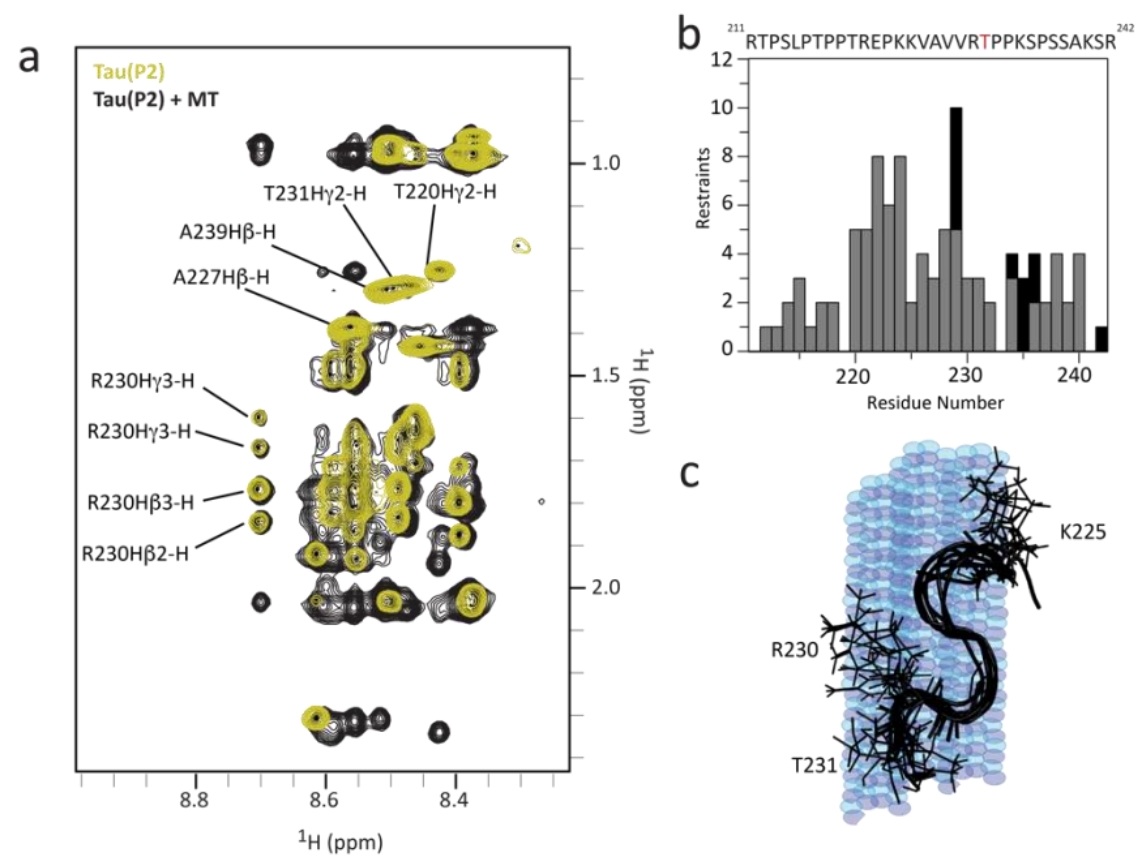

Figure 5.16. Structure of the proline-rich region of Tau bound to MTs. a) Superposition of 2D NOESY spectra of $1 \mathrm{mM} \operatorname{tau}(\mathrm{P} 2)$ in the absence (yellow) and presence of $50 \mu \mathrm{M}$ microtubule (black). The NOE mixing time was $100 \mathrm{~ms}$. b) Distribution of distance constraints as a function of residue number in tau(P2) upon addition of microtubules; at the bottom of the plot are the residue numbers, on the top is the amino acidic sequence of the peptide. Distance constraints were classified as medium-range $(1<|\mathrm{i}-\mathrm{j}| \leq 4$; grey) and long-range ( $\mid \mathrm{i}-$ $\mathrm{j} \mid \geq 5$; black), respectively. c) Statistics for the final conformers of microtubules-bound tau(P2). d) Conformation adopted by tau(P2) upon binding to microtubules: ensemble of the 10 lowest-energy conformers, residues 225-231 aligned, is shown; conformers were calculated on the basis of transferred NOEs observed within tau(P2) in the presence of microtubules (schematically shown in the background).

This demonstrates that upon binding of $\operatorname{Tau}(\mathrm{P} 2)$ to microtubules the conformational heterogeneity of the peptide is decreased. The structure-specific contacts between protons, which are less than $\sim 0.6 \mathrm{~nm}$ apart, are stable on the NOE time scale. Residue-specific analysis showed that the intra-molecular long-range contacts in presence of microtubules connected V229 with P233-S235 of Tau(P2) (Figure 5.16b). The medium- and long-range NOEs observed within Tau(P2) in presence of 
microtubules (Figure 5.16b and Table 5.2) were further used to calculate the structural ensemble adopted by Tau(P2) in the microtubule-bound state (Figure 5.16c). A single conformation for Tau(P2) could not be identified, which was not surprising, since previous NMR binding studies and mutational analysis reported that only part of the P2 region contributes to the interaction with tubulin/microtubules ${ }^{56,105}$. Within the functionally important region 224-237, however, the NMR-based structures clustered around a common conformation (Figure 5.16c). The data shown here indicate that the P2 domain in the proline-rich region of tau locally adopts a stable conformation upon binding to tubulin molecules.

Table 5.2 Structural statistics for the conformation of Tau(P2) bound to microtubules.

\begin{tabular}{|c|c|}
\hline $\begin{array}{l}\text { Structural statistics for the } 20 \text { final conformers of } \\
\text { MT-bound Tau peptide }\end{array}$ & $\begin{array}{l}\text { Tau }(211-242) \\
\text { (32 residues) }\end{array}$ \\
\hline Number of restraints & 321 \\
\hline Intra residue NOE $(|i-j|=0)$ & 134 \\
\hline Sequential $\operatorname{NOE}(|i-j|=1)$ & 136 \\
\hline Medium range NOE $(1<|i-j|<5)$ & 45 \\
\hline Long range NOE $(|i-j| \quad \geq 5)$ & 6 \\
\hline NOE violations $>0.5 \AA /$ structure & 0 \\
\hline \multicolumn{2}{|l|}{ Ramachandran plot statistics } \\
\hline Residues in most favored regions (\%) & 60.0 \\
\hline Residues in additionally allowed regions (\%) & 27.0 \\
\hline Residues in generously allowed regions (\%) & 9.6 \\
\hline Residues in disallowed regions $(\%)$ & 3.4 \\
\hline \multicolumn{2}{|l|}{ RMS deviations from the average structure } \\
\hline Backbone atoms $(\AA)$ & 0.78 \\
\hline Heavy atoms $(\AA)$ & 1.75 \\
\hline \multirow[t]{7}{*}{ (Residue range) } & $(224-227)$ \\
\hline & 0.72 \\
\hline & 1.48 \\
\hline & $(229-237)$ \\
\hline & 1.39 \\
\hline & 2.38 \\
\hline & $(224-237)$ \\
\hline
\end{tabular}

\subsection{Effect of Tau(P2) phosphorylation atT231 on the interaction with tubulin/microtubules}

As shown in the result section 5.3, the residues S202, T205, S210, T220, T231 and S235 in the proline-rich region P2 of tau are phosphorylated by Cdk2/CycA (Figure 5.3a). The phosphorylation in this region blocked LLPS-mediated tubulin polymerization (Figure 5.9). The residues T231 and S235 are located in the region that folds into a stable structure upon binding to microtubules (Figure 5.16c). The phosphorylation on T231 is recognized by the monoclonal antibody AT180 ${ }^{48}$ and has been found in the CSF of AD patients ${ }^{113}$. To gain insight into why Cdk2/CycA-phosphorylation blocks LLPS-mediated tubulin polymerization, a Tau(P2) peptide that is phosphorylated at T231 was prepared by solidphase synthesis (Figure 5.17a). 
STD NMR experiments recorded on Tau(P2)-T231p revealed that magnetization transfer was less efficient (Figure 5.17b and c), indicative of a slight decrease in tubulin affinity of Tau(P2)-T231p. The quantification of the saturation transfer as a function of Tau(P2)-T231p concentration showed a change in $K_{D}$ values, which were of $\sim 5 \mathrm{mM}$ ( Figure 5.18b and Table 5.1).

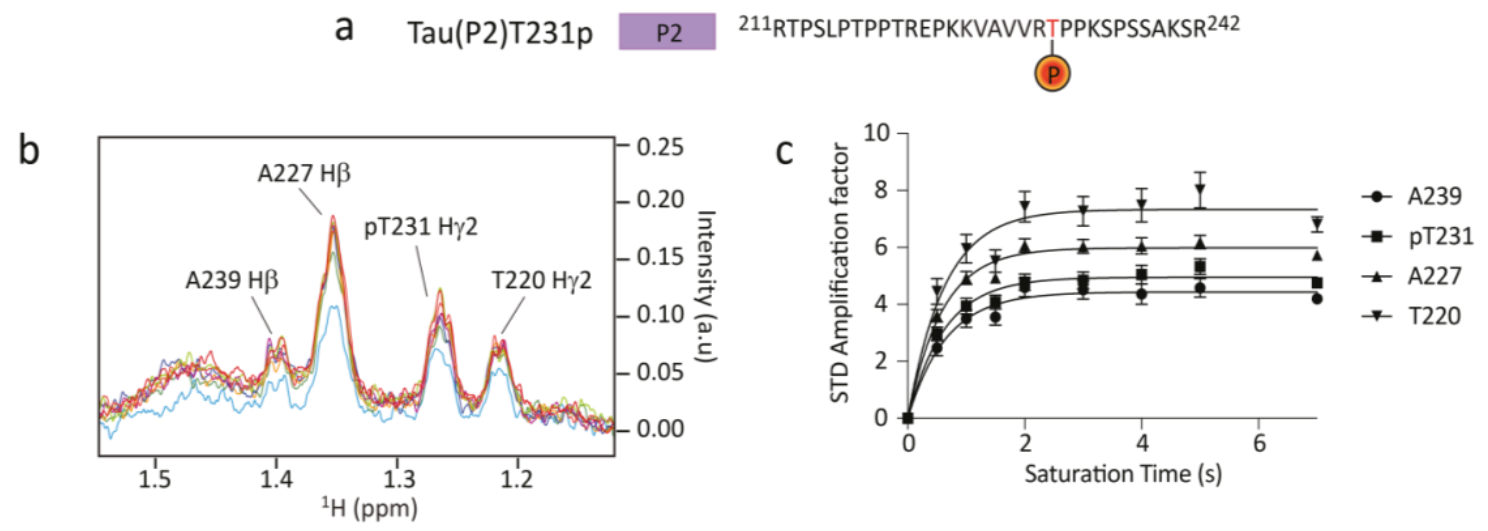

Figure 5.17.STD NMR on Tau(P2)-T231p.a) Schematic representation of the amino acid sequence of Tau(P2), the phosphorylation of threonine 231 is marked in red. b) STD NMR buildup curve performed at $5{ }^{\circ} \mathrm{C}$ on Tau(P2)-T231p (800 $\mu \mathrm{M}$ ) in presence of $5 \mu \mathrm{M}$ of soluble tubulin in non-polymerizing BRB80 buffer ( $80 \mathrm{mM}$ Pipes, pH 6.8, $1 \mathrm{mM} \mathrm{MgSO}_{4}, 1 \mathrm{mM}$ EDTA, $1 \mathrm{mM}$ DTT); irradiation times of 0.5, 1, 1.5, 2, 3, 4, 5 and 7 seconds were used. Measurements were performed at $5{ }^{\circ} \mathrm{C}$ and in non-polymerizing BRB80 buffer ( $80 \mathrm{mM}$ Pipes, $\mathrm{pH}$ 6.8, $1 \mathrm{mM} \mathrm{MgSO}_{4}, 1 \mathrm{mM}$ EDTA, $1 \mathrm{mM} \mathrm{DTT)}$. c) STD buildup curves obtained by measurement of ${ }^{1} \mathrm{H}$ 1D STD spectra at different irradiation times. Measurements were performed at $5{ }^{\circ} \mathrm{C}$ in a non-polymerizing BRB80 buffer ( 80 mM Pipes, pH 6.8, 1 mM MgSO 1 , 1 mM EDTA, 1 mM DTT). Error bars were calculated according to the spectral signal-to-noise ratio.

Despite the only modest change in affinity, the acquisition of $2 \mathrm{D} \operatorname{trNOE}$ experiments on Tau(P2)-T231p in presence of microtubules revealed no appearance of additional NOE cross peaks (Figure 5.18c). This lead to the conclusion that the phosphorylation at T231 does not inhibit binding of tau to tubulin, consistent with the ability of Cdk2/CyCA-phosphorylated tau to bind to tubulin and microtubules. Instead phosphorylation at $\mathrm{T} 231$ blocks a conformational change in the proline-rich region $\mathrm{P} 2$ that is required for LLPS-mediated nucleation of microtubule bundles. 
a

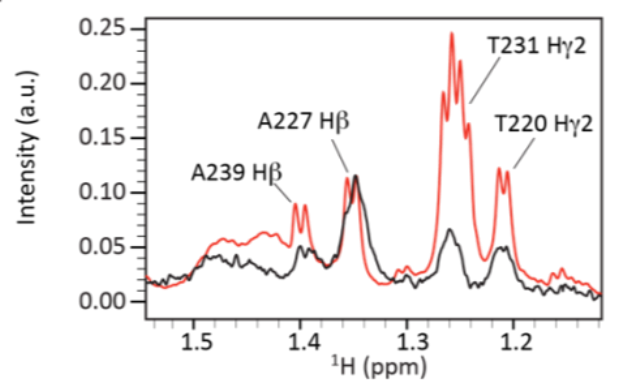

b

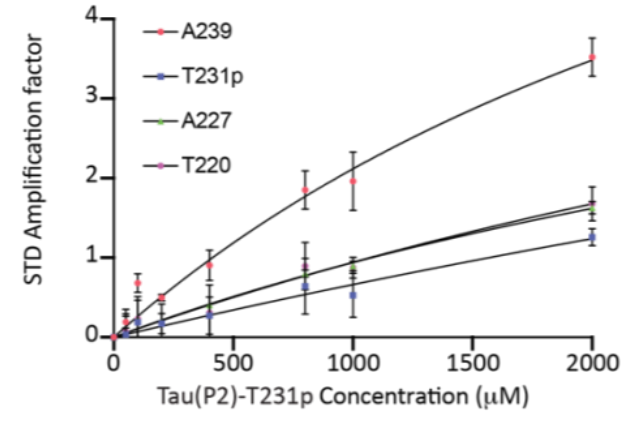

Tau(P2)-T231p

Tau(P2)-T231p+ microtubules

C

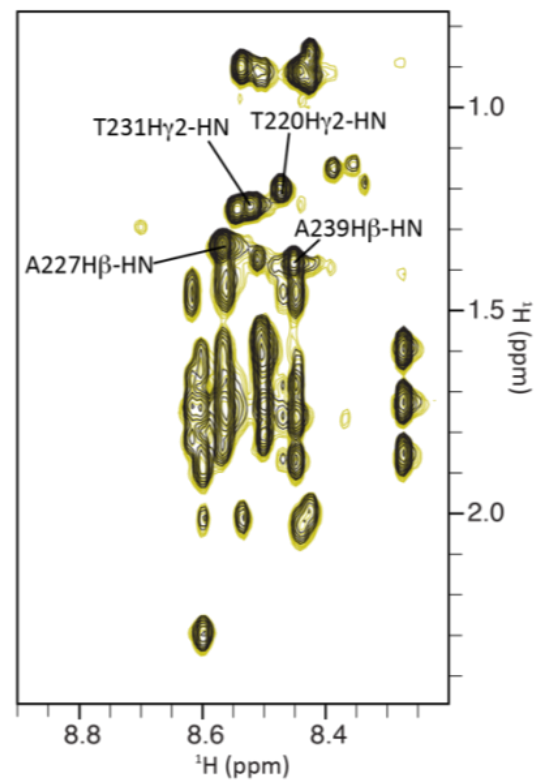

Figure 5.18.Effect of the phosphorylation of T231 on Tau(P2).a) Superposition of ${ }^{1} \mathrm{H} 1 \mathrm{D}$ spectrum (red) and ${ }^{1} \mathrm{H}$ 1D STD spectrum (black) of Tau(P2)-T231p, the proton resonances assignment for the selected residues is indicated. b) Quantification of tau(P2) saturation transfer as a function of the peptide concentration. The $K_{D}$ values were calculated by titrating increasing concentration of Tau(P2)-T231p to a fixed concentration of tubulin $(5 \mu \mathrm{M})$. Measurements were performed at $5{ }^{\circ} \mathrm{C}$ in non-polymerizing BRB80 Buffer (80 mM Pipes, pH 6.8, $1 \mathrm{mM} \mathrm{MgSO}_{4}, 1 \mathrm{mM}$ EDTA, $\left.1 \mathrm{mM} \mathrm{DTT}\right)$. Error bars were calculated on the basis of the spectral signalto-noise ratio. c) Superposition of 2D NOESY spectra of $1 \mathrm{mM}$ tau(P2) in the absence (yellow) and presence of $50 \mu \mathrm{M}$ microtubule (black). The NOE mixing time was $100 \mathrm{~ms}$.

\subsection{Structural changes induced by phosphorylation in the proline-rich region of tau}

It has been previously reported that upon phosphorylation of T231 a salt bridge with its preceding R230 is formed ${ }^{49}$. To observe whether this salt bridge was also present in pTau(Cdk2/CycA2) a $2 \mathrm{D}^{1} \mathrm{H}-{ }^{15} \mathrm{~N}$ HSQC was acquired on $80 \mu \mathrm{M}$ of unmodified htau40 and pTau(Cdk2/CycA2). To probe the changes in the arginine side chain region of the spectrum, a spectral width of $8.5 \mathrm{ppm}$ and $2 \mathrm{ppm}$ for ${ }^{1} \mathrm{H}$ and ${ }^{15} \mathrm{~N}$, respectively, was used. In order to mitigate the exchange of the labile side-chain guanidinium protons, a buffer at $\mathrm{pH} 6$ instead of the usual pH 6.8 was used. In the spectrum of htau40, a large cluster of 
unresolved signals together with a broad, slightly-downfield shifted signal was detected (Figure 5.19b).

In addition to the cluster of unresolved signals, new sharp, downshifted signals appeared in the spectrum of pTau(Cdk2) (Figure 5.19b). In order to determine the identity of these new peaks, a tau peptide phosphorylated at T231 and S235 and comprising residues 225-246 was used. $2 \mathrm{pTau}(225-246)$ comprises the sequence ${ }^{225}$ KVAVVRTPPKSPSSAKSRLQTA ${ }^{246}$ of the proline-rich region. This peptide was selected, because it contains the tubulin-binding region and only two arginine residues, i.e. R230 and R242, which facilitated their identification. By superposition of the spectrum of $2 \mathrm{pTau}(225-246)$ withthat of $\mathrm{pTau}(\mathrm{Cdk} 2 / \mathrm{CycA2})$ the two arginine residues present in $2 \mathrm{pTau}(225-246)$ were located in the broad cluster of guanidinium proton resonances, which are not involved in salt bridges, and in the sharp signal at $~ 7.5 \mathrm{ppm}$, respectively (Figure 5.19). Residue-specific assignment identified this signal as that of the $\mathrm{H} \varepsilon$ of $\mathrm{R} 230^{49}$. In conclusion, the observation that only $\mathrm{R} 230$ but not $\mathrm{R} 242$ is involved in a salt bridge suggests that the htau40, salt bridges are preferentially formed between phosphorylated residues and directly preceding arginine residues such as R230/T231. The combined data demonstrate that phosphorylation of serine/threonine residues in the proline-rich region of tau results in formation of intramolecular salt bridges between the phosphate groups and the side-chain guanidinium protons of arginine residues.

a

MAEPRQEFEVMEDHAGTYGL GDRKDQGGYTMHQDQEGDTD AGLKESPLQT

PTEDGSEEPG SETSDAKSTP TAEDVTAPLV DEGAPGKQAA AQPHTEIPEG

TTAEEAGIGD TPSLEDEAAG HVTQARMVSK SKDGTGSDDKKAKGADGKTK \begin{tabular}{lll}
11 & 198 \\
\hline IATPRGAAPP GQKGQANATR IPAKTPPAPK TPPSSGEPPK SGDRSGYSSP
\end{tabular} GSPGTPGSRS RTPSLPTPPT REPKKVAVVR TPPKSPSSAK SRLQTAPVPM PDLKNVKSKI GSTENLKHQP GGGKVQIINK KLDLSNVQSK CGSKDNIKHV

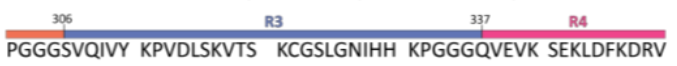

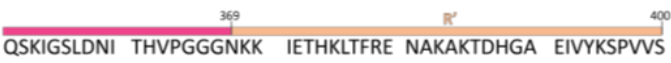
GDTSPRHLSN VSSTGSIDMV DSPQLATLAD EVSASLAKQG L b

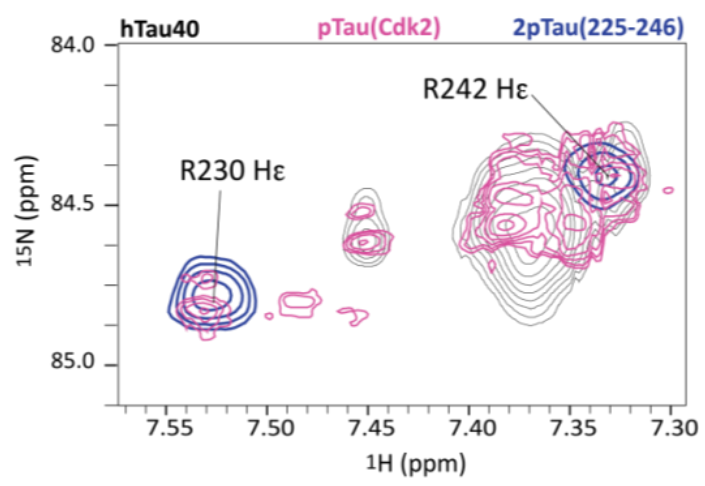

Figure 5.19. Structural impact of tau phosphorylation mediated by Cdk2/CycA2.a) Amino acid sequence of htau40 with domain organization. Serine and threonine residues phosphorylated by Cdk2/CycA2 are indicated by blue diamonds. The arginine residues (four in total) located in the P2 domain are highlighted in green. b) Superposition of the selected region of the ${ }^{1} \mathrm{H}^{15} \mathrm{~N}$ HSQC spectra of htau40 (black), pTau(Cdk2/CycA2) (pink) and 2pTau(225-246) (blue) for the guanidinium region of arginine residues. 
Results: project II

Depicted is the appearance of additional peaks for the guanidinium group of arginine residues due to the formation of a salt bridge with phosphorylated of threonine residues. 


\section{Discussion: project II}

The IDP tau has been intensively studied over the last decades due its direct connection with the onset with neurodegenerative diseases $35,36,125$. Because of its intrinsically disordered nature in solution, tau can interact with different partners (e.g. tubulin and actin) by adopting different conformations ${ }^{19,25}$. Regarding tau interaction with tubulin and the microtubules, it has been reported that the protein engages the P2 domain in the proline-rich region as well as the R1, R2 and the R3 pseudo-repeats ${ }^{52,56,98}$. The recent model obtained by cryo-EM of simplified tau constructs bound to microtubules could provide information regarding the pseudo-repeat region but no information on either the proline-rich region northe interrepeat PGGG motifs was provided ${ }^{101}$. A unified model to explain tau interaction with tubulin and microtubules is yet to be found.

\subsection{Tau interaction with paclitaxel-stabilized microtubules and soluble tubulin}

The loss of intensity upon addition of paclitaxel-stabilized microtubules/tubulin indicates that the exchange between tau microtubules/tubulin-bound and unbound conformation is intermediate on the NMR time scale ${ }^{197}$. In agreement with previously reported evidences, the NMR data presented in this work show that residues belonging to the P2 domain, the R1, R2 and R3 domains are engaged in the interaction with microtubules ${ }^{52,56,98}$ (Figure 5.1a). These regions are similarly involved in the binding to soluble tubulin (Figure 5.2).

The temperature dependence of the tau-microtubule interaction was investigated by measuring 2D ${ }^{1} \mathrm{H}-{ }^{15} \mathrm{~N}$ HSQC and $2 \mathrm{D}{ }^{1} \mathrm{H}_{-}{ }^{15} \mathrm{~N}$ TROSY experiments at three different temperatures, i.e. $5^{\circ} \mathrm{C}, 25^{\circ} \mathrm{C}$ and $37^{\circ} \mathrm{C}$. $2 \mathrm{D}$ NMR experiments were performed on $50 \mu \mathrm{M}$ htau40 in absence and presence of paclitaxel-stabilized microtubules. The NMR binding profiles obtained from the measurement of $2 \mathrm{D}{ }^{1} \mathrm{H}-{ }^{15} \mathrm{~N} \mathrm{HSQC}$ and $2 \mathrm{D}{ }^{1} \mathrm{H}-{ }^{15} \mathrm{~N}$ TROSY (Figure $5.1 \mathrm{~b}$ and $5.1 \mathrm{c}$ ) displayed loss of signal intensity for the same regions.

In both sets of experiments, the increase in htau40 concentration caused an expanded signal broadening, which involved regions in the $\mathrm{N}$ - and $\mathrm{C}$-terminal regions in addition to the P2, R1, R2 and R3 domains. For example, the residues S114-T123 in the N- 
terminal region and G164-G186 in the P1 domain displayed a significant loss of signal intensity. This observation suggested that at high tau-microtubule concentrations more tau regions are engaged in the binding. In particular, the $\alpha$-helix formed by the residue patch from L114 to T123, located before the proline-rich region, could provide additional contacts via its hydrophobic residues ${ }^{56}$

At $37^{\circ} \mathrm{C}$, the $\mathrm{P} 2$, the $\mathrm{R} 1$ and the $\mathrm{R} 3$ domains, remained involved in the interaction while the R2 domain exhibited an attenuated signal broadening, suggesting that upon increase of temperature its interaction with the microtubules is attenuated. Mukrasch et al., reported hydrophobicity values for the tau sequence ${ }^{56}$, according to which the residues ${ }^{225} \mathrm{KVAVVRT}^{231}$ (in P2), ${ }^{245} \mathrm{TAPVPMPDL}^{253}$ (in R1), ${ }^{275}$ VQIINKKLDLSNV ${ }^{287}$ (in R2) and ${ }^{306}$ VQIVYKPVDLSKV ${ }^{318}$ (in R3) showed positive values. These amino acid stretches contribute to the interaction with microtubules via hydrophobic interactions ${ }^{56,98}$. Since the hydrophobic force has a peak between $30{ }^{\circ} \mathrm{C}$ and $80{ }^{\circ} \mathrm{C}{ }^{198}$, these regions remain involved in the interaction with microtubules even as the temperature increases. Compared to the others, the ${ }^{275}$ VQIINKKLDLSNV ${ }^{287}$ sequence, in the R2 domain, is the least hydrophobic, which would explain the weakening of its interaction at $37^{\circ} \mathrm{C}$. It is noteworthy that these regions are positively charged (Figure 1.8), thus electrostatic interactions play together with hydrophobic interactions an important role in maintaining the binding with the microtubules.

\subsection{Tau phosphorylation in different regions of the sequence}

In this work it has been shown that phosphorylation of the proline-rich region of tau mediated by $\mathrm{Cdk} 2 / \mathrm{CycA} 2$ is responsible for the inhibition of the tubulin polymerization in tau phaseseparated conditions. Cdk2 is a member of the family of cyclin-dependent kinases like the ADrelated Cdk5 (see section 1.6) ${ }^{22}$. So far, no direct involvement of Cdk2 in AD has been reported, but via phosphorylation of serine and threonine residues in the proline-rich region it recreates the epitope recognized by the antibody AT180. To obtain a similar phosphorylation pattern, Cdk5 and GSK3 $\beta$ need to operate in a sequential manner ${ }^{48,112}$. This makes Cdk2 a practical enzyme for in vitro studies and it has been reported that tau phosphorylation with this kinase does not impair its binding to microtubules ${ }^{48}$. Phosphorylation of htau40 by incubation with Cdk2/CycA2 was validated using NMR 
spectroscopy, SDS-PAGE electrophoresis and mass spectrometry. The binding of pTau(Cdk2/CycA2) to microtubules/soluble tubulin was not inhibited and involved the P2 domain as well as the pseudo-repeats region (Figure 5.5b and 5.6b). Nevertheless, slight differences in the regions targeted by the kinase could be noted in the NMR binding profiles of htau40 and pTau(Cdk2/CycA2) in presence of microtubules/soluble tubulin.

In order to compare different phosphorylation patterns, tau was additionally phosphorylated using MARK2, which phosphorylates serine residues in the pseudo-repeat region ${ }^{118}$. Phosphorylation of htau40 by MARK2 is considered to inhibit the binding to microtubules and has been correlated to aggregation of tau ${ }^{90,118}$. In presence of paclitaxelstabilized microtubules, the NMR binding profile of pTau(MARK2) showed a weakened signal intensity loss, with major effects in the pseudo-repeats region. Mass spectrometry analysis further suggested a total of 9-10phosphorylation sites (Figure 5.10c), but only five cross peaks appeared with downfield shifts indicating the addition of the phosphate group. Despite the NMR and mass spectrometry analysis confirmed the phosphorylation, $\sim 50 \%$ of the targeted residues remained unphosphorylated (Figure 5.12). Consistent with a previous study by Schwalbe and collaborators ${ }^{118}$, the residues S262, S324 and S356 showed the highest degree of phosphorylation (Figure 5.11), and the regions surrounding these residues exhibited the strongest signal intensities in the NMR binding profile, as shown in figure 5.12. The residues S305 and S416 identified in the spectrum of pTau(MARK2) had a significant lower degree of phosphorylation (Figure 5.11), which might be explained by the fact that these residues are not located in the canonical consensus motif (KXGS) for MARK2. The residues S293, S352 and S416, which are in vitro phosphorylated by MARK2 $2^{118}$, could not be identified. Consistent with what so far discussed, it is possible that the phosphorylation reaction conditions, e.g. amounts of the kinase used, incubation time, might have not been sufficient to phosphorylated these residues.

\subsection{Tubulin polymerization in tau phase separation conditions}

The previous work by Ambadipudi and co-workers reported tau's ability to undergo LLPS in vitro ${ }^{70}$. This is due to the intrinsically disordered properties of tau, as well as the rich composition in positively charged residues. Arginine and lysine residues are important for 
the establishment of important interactions driving tau phase separation ${ }^{70,199}$. Hernández-Vega and co-workers reported that tubulin can be recruited into droplets of phase-separated tau and that in this environment tubulin efficiently polymerizes into microtubules ${ }^{71}$. In pTau(Cdk2/CycA2) phase separation conditions, tubulin polymerization did not occur, indicating that the recruitment in high tau-concentrated environment is not sufficient for the polymerization process to happen (Figure 5.9).

Such an effect was not observed when tau was phosphorylated with MARK2 (Figure 5.13). Because the phosphorylation mediated by MARK2 is located in the pseudorepeat region, this observation further supports the relevance of the proline-rich region in tubulin polymerization occurring under tau phase separation conditions.

\subsection{Interaction between the proline-rich region of tau and tubulin/microtubules}

In the models so far proposed to describe tau interaction with tubulin and microtubules, not much information is provided for the proline-rich region ${ }^{101,200}$. Being an IDP, tau adopts a wide spectrum of conformations and can interact with different partners. STD NMR and TrNOE are NMR methods optimized for the observation of dynamic protein interactions $^{166}$. STD NMR experiments performed on Tau(P2), comprising the ${ }^{225} \mathrm{KVAVVRT}^{231}$, reported binding affinity to tubulin with $\mathrm{K}_{\mathrm{D}}$ values 1-2 $\mathrm{mM}$ (Table 5.1). Despite this weak affinity, TrNOE experiments performed on Tau(P2) indicated that the proline-rich region adopted a stable structure upon binding to microtubules. In particular, residues belonging to the ${ }^{225} \mathrm{KVAVVRT}^{231}$ sequence were included in the structure. This amino acid stretch is the most hydrophobic in the $\mathrm{P} 2$ domain $^{56}$ and is important for the binding to tubulin/microtubules 56,105 .

By comparison of the structure presented in this work and other structures calculated for tau/tau constructs interacting with cytoskeleton elements, it is clear that different conformational rearrangements of the tau regions are responsible for the protein multivalency. The structure calculation for tau(267-312) peptide (PDB: 2MZ7) evidenced that residues 269-284 in the $\mathrm{R} 2$ and residues 300-311 in the R3 domains maintain flexibility when contacting microtubules ${ }^{100}$. This highlights the importance of transient interactions 
for supporting fast cytoskeleton rearrangements when tau contacts the microtubule surface. Cabrales et al. reported a three-dimensional structure of the residues 254-290 in the R2 domain bound to filamentous actin (F-actin) ${ }^{25}$ (PDB: 3JOS). In order to efficiently bind F-actin, this region adopts an $\alpha$-helical conformation.

\subsection{Effect of T231 phosphorylation on tau interaction with tubulin/microtubules}

T231 is located in the P2 domain, right at the end of the ${ }^{225} \mathrm{KVAVVRT}^{231}$ sequence. The phosphorylation atthis site has been correlated with early onset $A D$ and was found elevatedin CSF-tau ${ }^{113}$. The phosphorylation ofT231 did not drastically change the affinity of Tau(P2) for soluble tubulin (Figure 5.17 and table 5.1) but upon interaction with the microtubules the acquisition of the stable structure, observed in absence of phosphorylation, was inhibited (Figure 5.18).

Schwalbe and co-workers reported that upon phosphorylation of T231, a salt bridge is formed with the preceding $\mathrm{R} 230^{49}$. Consistent with this, in the $2 \mathrm{D}^{1} \mathrm{H}-{ }^{15} \mathrm{~N} H S Q C$ spectrum of tau phosphorylated by Cdk2/CycA2, the appearance of additional cross peaks was indicative of conformational changes (Figure 5.19b). It is possible that upon phosphorylation of htau40, the residues R230 and T231 form an intramolecular salt bridge, engaging the guanidinium side chain protons of R230 and the phosphate group of T231. The formation of this salt bridge is then responsible for restraining the conformation of residues 224-237, which become no longer available to establish contacts with soluble tubulin. In addition, the formation of a tau-tubulin complex might require the side chain of R230 for the formation of a salt bridge with residues on the tubulin surface. It is not to exclude that intermolecular salt bridges might be relevant for tau interaction with tubulin and that the arginine residues located in the P2 domain (Figure 5.19a) could serve for the establishment of more intermolecular salt bridges. Thus, the formation of intramolecular salt bridges could hamper the conformational transition of tau required for binding to tubulin and its polymerization into microtubules. 


\section{Bibliography}

1. Przedborski, S., Vila, M. \& Jackson-Lewis, V. Neurodegeneration: what is it and where are we? J Clin Invest111, 3-10 (2003).

2. Jellinger, K.A. Basic mechanisms of neurodegeneration: a critical update. I Cell Mol Med14, 457-87 (2010).

3. Chi, H., Chang, H.Y. \& Sang, T.K. Neuronal Cell Death Mechanisms in Major Neurodegenerative Diseases. Int J Mol Sci19(2018).

4. David L. Nelson, M.M.C. Lehninger Principles of Biochemistry, Seventh Edition 2017.

5. Sweeney, P. et al. Protein misfolding in neurodegenerative diseases: implications and strategies. Transl Neurodegener6, 6 (2017).

6. DiFiglia, M. et al. Aggregation of huntingtin in neuronal intranuclear inclusions and dystrophic neurites in brain. Science277, 1990-3 (1997).

7. Hane, F.T., Lee, B.Y. \& Leonenko, Z. Recent Progress in Alzheimer's Disease Research, Part 1: Pathology. J Alzheimers Dis57, 1-28 (2017).

8. Ahmad, S.I. Neurodegenerative diseases, xxxi, 393 p. (Landes Bioscience, New York Springer Science+Business Media ; Austin, Tex., 2012).

9. Brown, R.C., Lockwood, A.H. \& Sonawane, B.R. Neurodegenerative diseases: an overview of environmental risk factors. Environ Health Perspect113, 1250-6 (2005).

10. Association, A.s. Alzheimer's Association. 2019 Alzheimer's Disease Facts and Figures. Alzheimers Dement15, 321-87 (2019).

11. International, A.s.D. World Alzheimer Report 2019. Attitudes to dementia. (2019).

12. (ADI), A.s.D.I. World Alzheimer Report 2018. The state of the art of dementia research: New frontiers. (2018).

13. Solanki, I., Parihar, P. \& Parihar, M.S. Neurodegenerative diseases: From available treatments to prospective herbal therapy. Neurochem Int95, 100-8 (2016).

14. Somnath Pal, B.P., MBA, PhD. Selected Neurodegenerative Disorders. in US Pharmacist Vol. 376 (2012).

15. Kovacs, G.G. Tauopathies. Handb Clin Neuro/145, 355-368 (2017).

16. Mandelkow, E.M. \& Mandelkow, E. Biochemistry and cell biology of tau protein in neurofibrillary degeneration. Cold Spring Harb Perspect Med2, a006247 (2012).

17. Kolarova, M., Garcia-Sierra, F., Bartos, A., Ricny, J. \& Ripova, D. Structure and pathology of tau protein in Alzheimer disease. Int J Alzheimers Dis2012, 731526 (2012).

18. Binder, L.I., Frankfurter, A. \& Rebhun, L.I. The distribution of tau in the mammalian central nervous system. J Cell Bio/101, 1371-8 (1985).

19. Trinczek, B., Biernat, J., Baumann, K., Mandelkow, E.M. \& Mandelkow, E. Domains of tau protein, differential phosphorylation, and dynamic instability of microtubules. Mol Biol Cell6, 1887-902 (1995).

20. Wang, J.Z. \& Liu, F. Microtubule-associated protein tau in development, degeneration and protection of neurons. Prog Neurobio/85, 148-75 (2008).

21. Qiang, L. et al. Tau Does Not Stabilize Axonal Microtubules but Rather Enables Them to Have Long Labile Domains. Curr Bio/28, 2181-2189 e4 (2018).

22. Baumann, K., Mandelkow, E.M., Biernat, J., Piwnica-Worms, H. \& Mandelkow, E. Abnormal Alzheimer-like phosphorylation of tau-protein by cyclin-dependent kinases cdk2 and cdk5. FEBS Lett336, 417-24 (1993).

23. Martin, L. et al. Tau protein kinases: involvement in Alzheimer's disease. Ageing Res Rev12, 289-309 (2013).

24. Li, B., Chohan, M.O., Grundke-Iqbal, I. \& Iqbal, K. Disruption of microtubule network by Alzheimer abnormally hyperphosphorylated tau. Acta Neuropathol113, 501-11 (2007). 
25. Cabrales Fontela, Y. et al. Multivalent cross-linking of actin filaments and microtubules through the microtubule-associated protein Tau. Nat Commun8, 1981 (2017).

26. Arendt, T., Stieler, J.T. \& Holzer, M. Tau and tauopathies. Brain Res Bull126, 238-292 (2016).

27. Williams, D.R. Tauopathies: classification and clinical update on neurodegenerative diseases associated with microtubule-associated protein tau. Intern Med J36, 652-60 (2006).

28. Lebouvier, T., Pasquier, F. \& Buee, L. Update on tauopathies. Curr Opin Neuro/30, 589-598 (2017).

29. Alzheimer, A., Stelzmann, R.A., Schnitzlein, H.N. \& Murtagh, F.R. An English translation of Alzheimer's 1907 paper, "Uber eine eigenartige Erkankung der Hirnrinde". Clin Anat8, 429-31 (1995).

30. Robinson, M., Lee, B.Y. \& Hane, F.T. Recent Progress in Alzheimer's Disease Research, Part 2: Genetics and Epidemiology. J Alzheimers Dis57, 317-330 (2017).

31. Sahoo, A.K., Dandapat, J., Dash, U.C. \& Kanhar, S. Features and outcomes of drugs for combination therapy as multi-targets strategy to combat Alzheimer's disease. J Ethnopharmaco/215, 42-73 (2018).

32. Long, J.M. \& Holtzman, D.M. Alzheimer Disease: An Update on Pathobiology and Treatment Strategies. Cell (2019).

33. Lee, H.E., Lim, D., Lee, J.Y., Lim, S.M. \& Pae, A.N. Recent tau-targeted clinical strategies for the treatment of Alzheimer's disease. Future Med Chem11, 1845-1848 (2019).

34. Brion, J.P., Couck, A.M., Passareiro, E. \& Flament-Durand, J. Neurofibrillary tangles of Alzheimer's disease: an immunohistochemical study. J Submicrosc Cyto/17, 89-96 (1985).

35. Wischik, C.M. et al. Structural characterization of the core of the paired helical filament of Alzheimer disease. Proc Natl Acad Sci U S A85, 4884-8 (1988).

36. Wischik, C.M. et al. Isolation of a fragment of tau derived from the core of the paired helical filament of Alzheimer disease. Proc Natl Acad Sci U S A85, 4506-10 (1988).

37. Goedert, M., Wischik, C.M., Crowther, R.A., Walker, J.E. \& Klug, A. Cloning and sequencing of the CDNA encoding a core protein of the paired helical filament of Alzheimer disease: identification as the microtubule-associated protein tau. Proc Natl Acad Sci U S A85, 40515 (1988).

38. Grundke-Iqbal, I. et al. Microtubule-associated protein tau. A component of Alzheimer paired helical filaments. J Biol Chem261, 6084-9 (1986).

39. Braak, H. \& Braak, E. Staging of Alzheimer's disease-related neurofibrillary changes. Neurobiol Aging16, 271-8; discussion 278-84 (1995).

40. Cleveland, D.W., Hwo, S.Y. \& Kirschner, M.W. Purification of tau, a microtubule-associated protein that induces assembly of microtubules from purified tubulin. J Mol Bio/116, 20725 (1977).

41. Cleveland, D.W., Hwo, S.Y. \& Kirschner, M.W. Physical and chemical properties of purified tau factor and the role of tau in microtubule assembly. J Mol Bio/116, 227-47 (1977).

42. Grover, A. et al. 5' splice site mutations in tau associated with the inherited dementia FTDP-17 affect a stem-loop structure that regulates alternative splicing of exon 10. J Biol Chem274, 15134-43 (1999).

43. Neve, R.L., Harris, P., Kosik, K.S., Kurnit, D.M. \& Donlon, T.A. Identification of cDNA clones for the human microtubule-associated protein tau and chromosomal localization of the genes for tau and microtubule-associated protein 2. Brain Res387, 271-80 (1986).

44. Goedert, M., Spillantini, M.G., Potier, M.C., Ulrich, J. \& Crowther, R.A. Cloning and sequencing of the cDNA encoding an isoform of microtubule-associated protein tau containing four tandem repeats: differential expression of tau protein mRNAs in human brain. EMBO J8, 393-9 (1989). 
45. Takuma, H., Arawaka, S. \& Mori, H. Isoforms changes of tau protein during development in various species. Brain Res Dev Brain Res142, 121-7 (2003).

46. Melkova, K. et al. Structure and Functions of Microtubule Associated Proteins Tau and MAP2c: Similarities and Differences. Biomolecules9(2019).

47. Ball, L.J., Kuhne, R., Schneider-Mergener, J. \& Oschkinat, H. Recognition of proline-rich motifs by protein-protein-interaction domains. Angew Chem Int Ed Engl44, 2852-69 (2005).

48. Amniai, L. et al. Alzheimer disease specific phosphoepitopes of Tau interfere with assembly of tubulin but not binding to microtubules. FASEB J23, 1146-52 (2009).

49. Schwalbe, M. et al. Structural Impact of Tau Phosphorylation at Threonine 231. Structure23, 1448-1458 (2015).

50. Gustke, N., Trinczek, B., Biernat, J., Mandelkow, E.M. \& Mandelkow, E. Domains of tau protein and interactions with microtubules. Biochemistry33, 9511-22 (1994).

51. Barghorn, S. \& Mandelkow, E. Toward a unified scheme for the aggregation of tau into Alzheimer paired helical filaments. Biochemistry41, 14885-96 (2002).

52. Mukrasch, M.D. et al. The "jaws" of the tau-microtubule interaction. J Biol Chem282, 12230-9 (2007).

53. Tompa, P. Intrinsically disordered proteins: a 10-year recap. Trends Biochem Sci37, 50916 (2012).

54. Dunker, A.K. et al. Intrinsically disordered protein. J Mol Graph Mode/19, 26-59 (2001).

55. Jeganathan, S., von Bergen, M., Mandelkow, E.M. \& Mandelkow, E. The natively unfolded character of tau and its aggregation to Alzheimer-like paired helical filaments. Biochemistry47, 10526-39 (2008).

56. Mukrasch, M.D. et al. Structural polymorphism of 441-residue tau at single residue resolution. PLoS Biol7, e34 (2009).

57. Bertini, I., McGreevy, K.S. \& Parigi, G. NMR of biomolecules : towards mechanistic systems biology, xxviii, 612 p. (Wiley-VCH, Weinheim, Germany, 2012).

58. Hyman, A.A., Weber, C.A. \& Julicher, F. Liquid-liquid phase separation in biology. Annu Rev Cell Dev Bio/30, 39-58 (2014).

59. Ukmar-Godec, T., Wegmann, S. \& Zweckstetter, M. Biomolecular condensation of the microtubule-associated protein tau. Semin Cell Dev Biol (2019).

60. Brangwynne, C.P., Tompa P., Pappu, R.V. Polymer physics of intracellular phase transitions. nature physics11, 899-904 (2015).

61. Li, P. et al. Phase transitions in the assembly of multivalent signalling proteins. Nature483, 336-40 (2012).

62. Banani, S.F., Lee, H.O., Hyman, A.A. \& Rosen, M.K. Biomolecular condensates: organizers of cellular biochemistry. Nat Rev Mol Cell Bio/18, 285-298 (2017).

63. Brangwynne, C.P. Phase transitions and size scaling of membrane-less organelles. J Cell Bio/203, 875-81 (2013).

64. Lee, C.F., Brangwynne, C.P., Gharakhani, J., Hyman, A.A. \& Julicher, F. Spatial organization of the cell cytoplasm by position-dependent phase separation. Phys Rev Lett111, 088101 (2013).

65. Boyko, S., Qi, X., Chen, T.H., Surewicz, K. \& Surewicz, W.K. Liquid-liquid phase separation of tau protein: The crucial role of electrostatic interactions. J Biol Chem (2019).

66. Ukmar-Godec, T. et al. Lysine/RNA-interactions drive and regulate biomolecular condensation. Nat Commun10, 2909 (2019).

67. Burke, K.A., Janke, A.M., Rhine, C.L. \& Fawzi, N.L. Residue-by-Residue View of In Vitro FUS Granules that Bind the C-Terminal Domain of RNA Polymerase II. Mol Cel/60, 231-41 (2015).

68. Lin, Y., Protter, D.S., Rosen, M.K. \& Parker, R. Formation and Maturation of PhaseSeparated Liquid Droplets by RNA-Binding Proteins. Mol Cell60, 208-19 (2015). 
69. Nott, T.J. et al. Phase transition of a disordered nuage protein generates environmentally responsive membraneless organelles. Mol Cell57, 936-947 (2015).

70. Ambadipudi, S., Biernat, J., Riedel, D., Mandelkow, E. \& Zweckstetter, M. Liquid-liquid phase separation of the microtubule-binding repeats of the Alzheimer-related protein Tau. Nat Commun8, 275 (2017).

71. Hernandez-Vega, A. et al. Local Nucleation of Microtubule Bundles through Tubulin Concentration into a Condensed Tau Phase. Cell Rep20, 2304-2312 (2017).

72. Tan, R. et al. Microtubules gate tau condensation to spatially regulate microtubule functions. Nat Cell Bio/21, 1078-1085 (2019).

73. Siahaan, V. et al. Kinetically distinct phases of tau on microtubules regulate kinesin motors and severing enzymes. Nat Cell Bio/21, 1086-1092 (2019).

74. Wegmann, S. et al. Tau protein liquid-liquid phase separation can initiate tau aggregation. EMBO J37(2018).

75. Eisenberg, D.S. \& Sawaya, M.R. Structural Studies of Amyloid Proteins at the Molecular Level. Annu Rev Biochem86, 69-95 (2017).

76. Tycko, R. Amyloid polymorphism: structural basis and neurobiological relevance. Neuron86, 632-45 (2015).

77. Tycko, R. Physical and structural basis for polymorphism in amyloid fibrils. Protein Sci23, 1528-39 (2014).

78. Goedert, M., Falcon, B., Zhang, W., Ghetti, B. \& Scheres, S.H.W. Distinct Conformers of Assembled Tau in Alzheimer's and Pick's Diseases. Cold Spring Harb Symp Quant Biol (2019).

79. Fitzpatrick, A.W.P. et al. Cryo-EM structures of tau filaments from Alzheimer's disease. Nature547, 185-190 (2017).

80. Falcon, B. et al. Structures of filaments from Pick's disease reveal a novel tau protein fold. Nature561, 137-140 (2018).

81. Ramachandran, G. \& Udgaonkar, J.B. Understanding the kinetic roles of the inducer heparin and of rod-like protofibrils during amyloid fibril formation by Tau protein. J Biol Chem286, 38948-59 (2011).

82. Mukrasch, M.D. et al. Sites of tau important for aggregation populate $\{$ beta\}-structure and bind to microtubules and polyanions. J Biol Chem280, 24978-86 (2005).

83. Bibow, S. et al. The dynamic structure of filamentous tau. Angew Chem Int Ed Eng/50, 11520-4 (2011).

84. Daebel, V. et al. beta-Sheet core of tau paired helical filaments revealed by solid-state NMR. J Am Chem Soc134, 13982-9 (2012).

85. von Bergen, $M$. et al. Assembly of tau protein into Alzheimer paired helical filaments depends on a local sequence motif ((306)VQIVYK(311)) forming beta structure. Proc Natl Acad Sci U S A97, 5129-34 (2000).

86. Andronesi, O.C. et al. Characterization of Alzheimer's-like paired helical filaments from the core domain of tau protein using solid-state NMR spectroscopy. J Am Chem Soc130, 59228 (2008).

87. Goux, W.J. et al. The formation of straight and twisted filaments from short tau peptides. J Biol Chem279, 26868-75 (2004).

88. Horowitz, P.M., LaPointe, N., Guillozet-Bongaarts, A.L., Berry, R.W. \& Binder, L.I. Nterminal fragments of tau inhibit full-length tau polymerization in vitro. Biochemistry45, 12859-66 (2006).

89. Martin, L., Latypova, X. \& Terro, F. Post-translational modifications of tau protein: implications for Alzheimer's disease. Neurochem Int58, 458-71 (2011).

90. Biernat, J., Gustke, N., Drewes, G., Mandelkow, E.M. \& Mandelkow, E. Phosphorylation of Ser262 strongly reduces binding of tau to microtubules: distinction between PHF-like immunoreactivity and microtubule binding. Neuron11, 153-63 (1993). 
91. Barghorn, S. et al. Structure, microtubule interactions, and paired helical filament aggregation by tau mutants of frontotemporal dementias. Biochemistry39, 11714-21 (2000).

92. Gorsky, M.K., Burnouf, S., Dols, J., Mandelkow, E. \& Partridge, L. Acetylation mimic of lysine 280 exacerbates human Tau neurotoxicity in vivo. Sci Rep6, 22685 (2016).

93. Schweers, O., Mandelkow, E.M., Biernat, J. \& Mandelkow, E. Oxidation of cysteine-322 in the repeat domain of microtubule-associated protein tau controls the in vitro assembly of paired helical filaments. Proc Natl Acad Sci U S A92, 8463-7 (1995).

94. Landino, L.M., Skreslet, T.E. \& Alston, J.A. Cysteine oxidation of tau and microtubuleassociated protein- 2 by peroxynitrite: modulation of microtubule assembly kinetics by the thioredoxin reductase system. J Biol Chem279, 35101-5 (2004).

95. Margittai, M. \& Langen, R. Side chain-dependent stacking modulates tau filament structure. J Biol Chem281, 37820-7 (2006).

96. Xiang, S. et al. A Two-Component Adhesive: Tau Fibrils Arise from a Combination of a WellDefined Motif and Conformationally Flexible Interactions. J Am Chem Soc139, 2639-2646 (2017).

97. Zhang, W. et al. Heparin-induced tau filaments are polymorphic and differ from those in Alzheimer's and Pick's diseases. Elife8(2019).

98. Kadavath, $\mathrm{H}$. et al. Tau stabilizes microtubules by binding at the interface between tubulin heterodimers. Proc Natl Acad Sci U S A112, 7501-6 (2015).

99. Butner, K.A. \& Kirschner, M.W. Tau protein binds to microtubules through a flexible array of distributed weak sites. J Cell Bio/115, 717-30 (1991).

100. Kadavath, H. et al. Folding of the Tau Protein on Microtubules. Angew Chem Int Ed Eng/54, 10347-51 (2015).

101. Kellogg, E.H. et al. Near-atomic model of microtubule-tau interactions. Science360, 12421246 (2018).

102. Nogales, E. Structural insights into microtubule function. Annu Rev Biochem69, 277-302 (2000).

103. Vleugel, M., Kok, M. \& Dogterom, M. Understanding force-generating microtubule systems through in vitro reconstitution. Cell Adh Migr10, 475-494 (2016).

104. Mitchison, T. \& Kirschner, M. Dynamic instability of microtubule growth. Nature312, 23742 (1984).

105. Goode, B.L. et al. Functional interactions between the proline-rich and repeat regions of tau enhance microtubule binding and assembly. Mol Biol Cell8, 353-65 (1997).

106. Goode, B.L., Chau, M., Denis, P.E. \& Feinstein, S.C. Structural and functional differences between 3-repeat and 4-repeat tau isoforms. Implications for normal tau function and the onset of neurodegenetative disease. J Biol Chem275, 38182-9 (2000).

107. Goode, B.L. \& Feinstein, S.C. Identification of a novel microtubule binding and assembly domain in the developmentally regulated inter-repeat region of tau. J Cell Bio/124, 76982 (1994).

108. Kadavath, H. et al. The Binding Mode of a Tau Peptide with Tubulin. Angew Chem Int Ed Eng/57, 3246-3250 (2018).

109. Weingarten, M.D., Lockwood, A.H., Hwo, S.Y. \& Kirschner, M.W. A protein factor essential for microtubule assembly. Proc Natl Acad Sci U S A72, 1858-62 (1975).

110. Landrieu, I. et al. NMR spectroscopy of the neuronal tau protein: normal function and implication in Alzheimer's disease. Biochem Soc Trans38, 1006-11 (2010).

111. An, Y. et al. The nuclear GSK-3beta regulated post-transcriptional processing of mRNA through phosphorylation of SC35. Mol Cell Biochem451, 55-67 (2019).

112. Engmann, O. \& Giese, K.P. Crosstalk between Cdk5 and GSK3beta: Implications for Alzheimer's Disease. Front Mol Neurosci2, 2 (2009). 
113. Buerger, K. et al. CSF tau protein phosphorylated at threonine 231 correlates with cognitive decline in $\mathrm{MCl}$ subjects. Neurology59, 627-9 (2002).

114. Kidemet-Piskac, S. et al. Evaluation of cerebrospinal fluid phosphorylated tau231 as a biomarker in the differential diagnosis of Alzheimer's disease and vascular dementia. CNS Neurosci Ther24, 734-740 (2018).

115. Cho JH, J.G. Primed phosphorylation of tau at Thr231 by glycogen synthase kinase 3beta (GSK3beta) plays a critical role in regulating tau's ability to bind and stabilize microtubules. Journal of Neurochemistry88, 349-358 (2004).

116. Lefevre, J. et al. The $C$ terminus of tubulin, a versatile partner for cationic molecules: binding of Tau, polyamines, and calcium. J Biol Chem286, 3065-78 (2011).

117. Serrano, L., Montejo de Garcini, E., Hernandez, M.A. \& Avila, J. Localization of the tubulin binding site for tau protein. Eur J Biochem153, 595-600 (1985).

118. Schwalbe, M. et al. Phosphorylation of human Tau protein by microtubule affinityregulating kinase 2. Biochemistry52, 9068-79 (2013).

119. Cohen, T.J. et al. The acetylation of tau inhibits its function and promotes pathological tau aggregation. Nat Commun2, 252 (2011).

120. Makrides, V., Massie, M.R., Feinstein, S.C. \& Lew, J. Evidence for two distinct binding sites for tau on microtubules. Proc Natl Acad Sci U S A101, 6746-51 (2004).

121. Melo, A.M. et al. A functional role for intrinsic disorder in the tau-tubulin complex. Proc Natl Acad Sci U S A113, 14336-14341 (2016).

122. Lee, V.M., Balin, B.J., Otvos, L., Jr. \& Trojanowski, J.Q. A68: a major subunit of paired helical filaments and derivatized forms of normal Tau. Science251, 675-8 (1991).

123. Alonso, A.D. et al. Phosphorylation of tau at Thr212, Thr231, and Ser262 combined causes neurodegeneration. J Biol Chem285, 30851-60 (2010).

124. Daly, N.L., Hoffmann, R., Otvos, L., Jr. \& Craik, D.J. Role of phosphorylation in the conformation of tau peptides implicated in Alzheimer's disease. Biochemistry39, 9039-46 (2000).

125. Grundke-lqbal, l. et al. Abnormal phosphorylation of the microtubule-associated protein tau (tau) in Alzheimer cytoskeletal pathology. Proc Natl Acad Sci U S A83, 4913-7 (1986).

126. Liu, F. et al. Site-specific effects of tau phosphorylation on its microtubule assembly activity and self-aggregation. Eur J Neurosci26, 3429-36 (2007).

127. Mercken, M. et al. Monoclonal antibodies with selective specificity for Alzheimer Tau are directed against phosphatase-sensitive epitopes. Acta Neuropatho/84, 265-72 (1992).

128. Cao, L. et al. Pseudo-phosphorylation at AT8 epitopes regulates the tau truncation at aspartate 421. Exp Cell Res370, 103-115 (2018).

129. Malia, T.J. et al. Epitope mapping and structural basis for the recognition of phosphorylated tau by the anti-tau antibody AT8. Proteins84, 427-34 (2016).

130. Amniai, L., Lippens, G. \& Landrieu, I. Characterization of the AT180 epitope of phosphorylated Tau protein by a combined nuclear magnetic resonance and fluorescence spectroscopy approach. Biochem Biophys Res Commun412, 743-6 (2011).

131. Hanger, D.P., Anderton, B.H. \& Noble, W. Tau phosphorylation: the therapeutic challenge for neurodegenerative disease. Trends Mol Med15, 112-9 (2009).

132. Kimura, T., Ishiguro, K. \& Hisanaga, S. Physiological and pathological phosphorylation of tau by Cdk5. Front Mol Neurosci7, 65 (2014).

133. Li, T., Hawkes, C., Qureshi, H.Y., Kar, S. \& Paudel, H.K. Cyclin-dependent protein kinase 5 primes microtubule-associated protein tau site-specifically for glycogen synthase kinase 3beta. Biochemistry45, 3134-45 (2006).

134. Hanger, D.P. \& Noble, W. Functional implications of glycogen synthase kinase-3-mediated tau phosphorylation. Int J Alzheimers Dis2011, 352805 (2011). 
135. Hernandez, F. et al. Glycogen synthase kinase-3 plays a crucial role in tau exon 10 splicing and intranuclear distribution of SC35. Implications for Alzheimer's disease. J Biol Chem279, 3801-6 (2004).

136. Yamaguchi, H. et al. Preferential labeling of Alzheimer neurofibrillary tangles with antisera for tau protein kinase (TPK) 1/glycogen synthase kinase-3 beta and cyclin-dependent kinase 5, a component of TPK II. Acta Neuropatho/92, 232-41 (1996).

137. Timm, T., Marx, A., Panneerselvam, S., Mandelkow, E. \& Mandelkow, E.M. Structure and regulation of MARK, a kinase involved in abnormal phosphorylation of Tau protein. BMC Neurosci9 Suppl 2, S9 (2008).

138. Schlatterer, S.D., Acker, C.M. \& Davies, P. c-Abl in neurodegenerative disease. J Mol Neurosci45, 445-52 (2011).

139. Derkinderen, P. et al. Tyrosine 394 is phosphorylated in Alzheimer's paired helical filament tau and in fetal tau with c-Abl as the candidate tyrosine kinase. J Neurosci25, 6584-93 (2005).

140. Jing, Z., Caltagarone, J. \& Bowser, R. Altered subcellular distribution of c-Abl in Alzheimer's disease. J Alzheimers Dis17, 409-22 (2009).

141. Vega, I.E. et al. Increase in tau tyrosine phosphorylation correlates with the formation of tau aggregates. Brain Res Mol Brain Res138, 135-44 (2005).

142. Cohen, S.S. \& Lichtenstein, J. The isolation of deoxyribonucleic acid from bacterial extracts by precipitation with streptomycin. J Biol Chem235, PC55-PC56 (1960).

143. Wingfield, P. Protein precipitation using ammonium sulfate. Curr Protoc Protein SciAppendix 3, Appendix 3F (2001).

144. Anthis, N.J. \& Clore, G.M. Sequence-specific determination of protein and peptide concentrations by absorbance at $205 \mathrm{~nm}$. Protein Sci22, 851-8 (2013).

145. Whitmore, L. \& Wallace, B.A. Protein secondary structure analyses from circular dichroism spectroscopy: methods and reference databases. Biopolymers89, 392-400 (2008).

146. Kelly, S.M., Jess, T.J. \& Price, N.C. How to study proteins by circular dichroism. Biochim Biophys Acta1751, 119-39 (2005).

147. Xue, C., Lin, T.Y., Chang, D. \& Guo, Z. Thioflavin T as an amyloid dye: fibril quantification, optimal concentration and effect on aggregation. $R$ Soc Open Sci4, 160696 (2017).

148. Markwick, P.R., Malliavin, T. \& Nilges, M. Structural biology by NMR: structure, dynamics, and interactions. PLoS Comput Bio/4, e1000168 (2008).

149. Levitt, M.H. Spin Dynamics: Basics of Nuclear Magnetic Resonance, 2nd edition. (2008).

150. Keeler, J. Understanding NMR spectroscopy, xiii, 511 p. (John Wiley and Sons, Chichester, U.K., 2010).

151. Hore, P.J. Nuclear magnetic resonance, viii, 112 pages (Oxford University Press, Oxford, 2015).

152. Michael Sattler, J.S., Christian Griesinger. Heteronuclear multidimensional NMR experiments for the structure determination of proteins in solution employing pulsed field gradients. Progress in Nuclear Magnetic Resonance Spectroscopy 34 93-158 (1998).

153. Morris G. A., F.R. Enhancement of Nuclear Magnetic Resonance Signals by Polarization Transfer. Journal of the American Chemical Society101, 760-762 (1978).

154. Lee, W., Tonelli, M. \& Markley, J.L. NMRFAM-SPARKY: enhanced software for biomolecular NMR spectroscopy. Bioinformatics31, 1325-7 (2015).

155. Vranken, W.F. et al. The CCPN data model for NMR spectroscopy: development of a software pipeline. Proteins59, 687-96 (2005).

156. Delaglio, F. et al. NMRPipe: a multidimensional spectral processing system based on UNIX pipes. J Biomol NMR6, 277-93 (1995).

157. Pervushin, K., Riek, R., Wider, G. \& Wuthrich, K. Attenuated T2 relaxation by mutual cancellation of dipole-dipole coupling and chemical shift anisotropy indicates an avenue 
to NMR structures of very large biological macromolecules in solution. Proc Natl Acad Sci U S A94, 12366-71 (1997).

158. Cavanagh, J. Protein NMR spectroscopy : principles and practice, xxv, 885 p. (Academic Press, Amsterdam ; Boston, 2007).

159. Fernandez, C. \& Wider, G. TROSY in NMR studies of the structure and function of large biological macromolecules. Curr Opin Struct Bio/13, 570-80 (2003).

160. Neuhaus, D. \& Williamson, M.P. The nuclear Overhauser effect in structural and conformational analysis, xxvii, 619 p. (Wiley, New York, 2000).

161. O'Connell, M.R., Gamsjaeger, R. \& Mackay, J.P. The structural analysis of protein-protein interactions by NMR spectroscopy. Proteomics9, 5224-32 (2009).

162. Clore, G.M. \& Gronenborn, A.M. Theory and Applications of the Transferred Nuclear Overhauser Effect to the Study of the Conformations of Small Ligands Bound to Proteins. J Magn Reson48, 402-417 (1982).

163. Schwieters, C.D., Kuszewski, J.J., Tjandra, N. \& Clore, G.M. The Xplor-NIH NMR molecular structure determination package. J Magn Reson160, 65-73 (2003).

164. Koradi, R., Billeter, M. \& Wuthrich, K. MOLMOL: a program for display and analysis of macromolecular structures. J Mol Graph14, 51-5, 29-32 (1996).

165. Lepre, C.A., Moore, J.M. \& Peng, J.W. Theory and applications of NMR-based screening in pharmaceutical research. Chem Rev104, 3641-76 (2004).

166. Meyer, B. \& Peters, T. NMR spectroscopy techniques for screening and identifying ligand binding to protein receptors. Angew Chem Int Ed Eng/42, 864-90 (2003).

167. Mayer, M. \& Meyer, B. Characterization of Ligand Binding by Saturation Transfer Difference NMR Spectroscopy. Angew Chem Int Ed Eng/38, 1784-1788 (1999).

168. Li, Y. \& Kang, C. Solution NMR Spectroscopy in Target-Based Drug Discovery. Molecules22(2017).

169. A. Viegas, J.M., F. L. Nobrega, E. J. Cabrita. Saturation-Transfer Difference (STD) NMR: A Simple and Fast Method for Ligand Screening and Characterization of Protein Binding. J. Chem. Educ88 (7), 990-994 (2011).

170. Jones J. A., W.D.K., Smith L. J., Dobson C. M. Characterisation of protein unfolding by NMR diffusion measurements. Journal of Biomolecular NMR10, 199-203 (1997).

171. Nygaard, M., Kragelund, B.B., Papaleo, E. \& Lindorff-Larsen, K. An Efficient Method for Estimating the Hydrodynamic Radius of Disordered Protein Conformations. Biophys J113, 550-557 (2017).

172. jr, J.C.S. Diffusion ordered nuclear magnetic resonance spectroscopy: principles and applications. Progress in Nuclear Magnetic Resonance Spectroscopy34 203-256 (1999).

173. Zheng, G., Stait-Gardner, T., Anil Kumar, P.G., Torres, A.M. \& Price, W.S. PGSTEWATERGATE: an STE-based PGSE NMR sequence with excellent solvent suppression. $J$ Magn Reson191, 159-63 (2008).

174. Wilkins, D.K. et al. Hydrodynamic radii of native and denatured proteins measured by pulse field gradient NMR techniques. Biochemistry38, 16424-31 (1999).

175. Duer, M.J. Introduction to solid-state NMR spectroscopy, xiv, 349 p. (Blackwell, Oxford, UK ; Malden, MA, 2004).

176. Szeverenyi N.M., S.M.J., Maciel G.E. . Observation of spin exchange by two-dimensional fourier transform $13 \mathrm{C}$ cross polarization-magic-angle spinning. Journal of Magnetic Resonance 47, 462-475 (1969).

177. Grommek A., M.B.H., Ernst M. Distance information from proton-driven spin diffusion under MAS. Chemical Physics Letters427, 404-409 (2006).

178. Fyfe C. A., W.-M.K.C., Huang Y., Grondey H. INEPT experiments in Solid-State NMR. Journal of American Chemical Society117, 10397-10398 (1995).

179. Andronesi, O.C. et al. Determination of membrane protein structure and dynamics by magic-angle-spinning solid-state NMR spectroscopy. J Am Chem Soc127, 12965-74 (2005). 
180. Falk, A.S. \& Siemer, A.B. Dynamic domains of amyloid fibrils can be site-specifically assigned with proton detected 3D NMR spectroscopy. J Biomol NMR66, 159-162 (2016).

181. Linser, R., Fink, U. \& Reif, B. Proton-detected scalar coupling based assignment strategies in MAS solid-state NMR spectroscopy applied to perdeuterated proteins. J Magn Reson193, 89-93 (2008).

182. Zhu, H.L. et al. Quantitative characterization of heparin binding to Tau protein: implication for inducer-mediated Tau filament formation. J Biol Chem285, 3592-9 (2010).

183. Fritzsching, K.J., Yang, Y., Schmidt-Rohr, K. \& Hong, M. Practical use of chemical shift databases for protein solid-state NMR: 2D chemical shift maps and amino-acid assignment with secondary-structure information. J Biomol NMR56, 155-67 (2013).

184. Pan, H., Barany, G. \& Woodward, C. Reduced BPTI is collapsed. A pulsed field gradient NMR study of unfolded and partially folded bovine pancreatic trypsin inhibitor. Protein Sci6, 1985-92 (1997).

185. Leite, J.P.G., L. Fluorescence properties of the amyloid indicator dye thioflavin $T$ in constrained environments. Fluorescence properties of the amyloid indicator dye thioflavin $T$ in constrained environments 160, 64-70 (2019).

186. Dregni, A.J. et al. In vitro ON4R tau fibrils contain a monomorphic beta-sheet core enclosed by dynamically heterogeneous fuzzy coat segments. Proc Natl Acad Sci U S A (2019).

187. Pascual, G. et al. Immunological memory to hyperphosphorylated tau in asymptomatic individuals. Acta Neuropatho/133, 767-783 (2017).

188. Zhang, H. et al. Structural Basis for Recognition of a Unique Epitope by a Human Anti-tau Antibody. Structure26, 1626-1634 e4 (2018).

189. Whitmore, L. \& Wallace, B.A. DICHROWEB, an online server for protein secondary structure analyses from circular dichroism spectroscopic data. Nucleic Acids Res32, W66873 (2004).

190. Zhukareva, V. et al. Sporadic Pick's disease: a tauopathy characterized by a spectrum of pathological tau isoforms in gray and white matter. Ann Neuro/51, 730-9 (2002).

191. Dasari, A.K.R., Kayed, R., Wi, S. \& Lim, K.H. Tau Interacts with the C-Terminal Region of alpha-Synuclein, Promoting Formation of Toxic Aggregates with Distinct Molecular Conformations. Biochemistry (2019).

192. von Bergen, M., Barghorn, S., Biernat, J., Mandelkow, E.M. \& Mandelkow, E. Tau aggregation is driven by a transition from random coil to beta sheet structure. Biochim Biophys Acta1739, 158-66 (2005).

193. Sherman, E. \& Haran, G. Coil-globule transition in the denatured state of a small protein. Proc Natl Acad Sci U S A103, 11539-43 (2006).

194. Seidler, P.M. et al. Structure-based inhibitors of tau aggregation. Nat Chem10, 170-176 (2018).

195. Li, W. et al. The EMBL-EBI bioinformatics web and programmatic tools framework. Nucleic Acids Res43, W580-4 (2015).

196. Goedert, M. et al. Epitope mapping of monoclonal antibodies to the paired helical filaments of Alzheimer's disease: identification of phosphorylation sites in tau protein. Biochem J301 ( Pt 3), 871-7 (1994).

197. Williamson, M.P. Using chemical shift perturbation to characterise ligand binding. Prog Nucl Magn Reson Spectrosc73, 1-16 (2013).

198. van Dijk, E., Hoogeveen, A. \& Abeln, S. The hydrophobic temperature dependence of amino acids directly calculated from protein structures. PLoS Comput Bio/11, e1004277 (2015).

199. Chong, P.A., Vernon, R.M. \& Forman-Kay, J.D. RGG/RG Motif Regions in RNA Binding and Phase Separation. J Mol Bio/430, 4650-4665 (2018).

200. Li, X.H. \& Rhoades, E. Heterogeneous Tau-Tubulin Complexes Accelerate Microtubule Polymerization. Biophys J112, 2567-2574 (2017). 
201. Wilkins, M.R. et al. Protein identification and analysis tools in the ExPASy server. Methods Mol Bio/112, 531-52 (1999). 


\section{APPENDIX}

\subsection{Tau parameters}

Table 9.1Tau Fasta sequence (from the uniprot entry: P10636).

\begin{tabular}{|c|c|c|c|c|}
\hline 10 & 20 & 30 & 40 & 50 \\
\hline MAE PRQEFEV & MEDHAGTYGL & GDRKDQGGYT & MHQDQEGDTD & AGLKESPLQT \\
\hline 60 & 70 & 80 & 90 & 100 \\
\hline PTEDGSEEPG & SETSDAKSTP & TAEDVTAPLV & DEGAPGKQAA & AQPHTEIPEG \\
\hline 110 & 120 & 130 & 140 & 150 \\
\hline TTAEEAGIGD & TPSLEDEAAG & HVTQARMVSK & SKDGTGSDDK & KAKGADGKTK \\
\hline 160 & 170 & 180 & 190 & 200 \\
\hline IAT PRGAAPP & GQKGQANATR & IPAKTPPAPK & TPPSSGEPPK & SGDRSGYSSP \\
\hline 210 & 220 & 230 & 240 & 250 \\
\hline GSP GTPGSRS & RTPSLPTPPT & REPKKVAVVR & TPPKSPSSAK & SRLQTAPVPM \\
\hline 260 & 270 & 280 & 290 & 300 \\
\hline PDLKNVKSKI & GSTENLKHQP & GGGKVQI INK & KLDLSNVQSK & CGSKDN IKHV \\
\hline 310 & 320 & 330 & 340 & 350 \\
\hline PGGGSVQIVY & KPVDLSKVTS & KCGSLGNIHH & KPGGGQVEVK & SEKLDFKDRV \\
\hline 360 & 370 & 380 & 390 & 400 \\
\hline QSKIGSLDNI & THVPGGGNKK & IETHKLTFRE & NAKAKTDHGA & EIVYKSPVVS \\
\hline 410 & 420 & 430 & 440 & \\
\hline GDTSPRHLSN & VSSTGSIDMV & DSPQLATLAD & EVSASLAKQG & $\mathrm{L}$ \\
\hline
\end{tabular}

\subsection{1 htau40 physical and chemical parameters}

$\begin{array}{ll}\text { Number of amino acids } & 441 \\ \text { Molecular weight (Da) } & 45849.91 \\ \text { Theoretical pl } & 8.24\end{array}$

These values can be obtained using the ExPAsy protparam software ${ }^{201}$.

\subsection{P2R2 and P2R3 polypeptide parameters}

Table 9.2 P2R2 FASTA sequence.

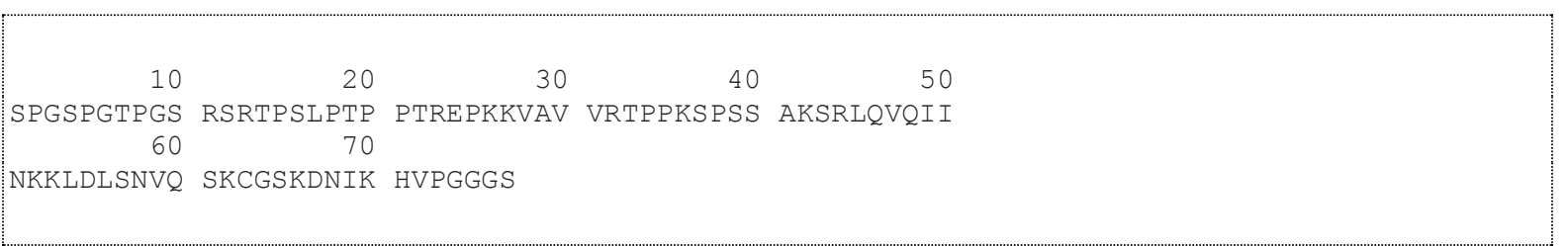

Table 9.3 P2R3 FASTA sequence.

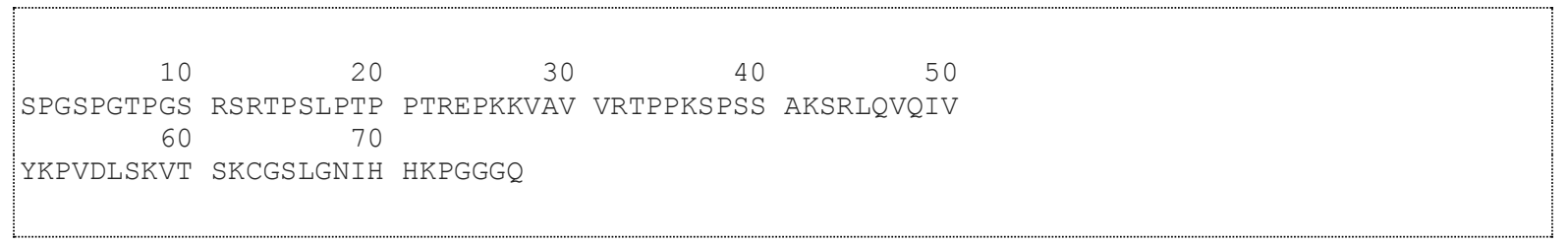


Table 9.4 P2R2 and P2R3 physical and chemical parameters.

$\begin{array}{lll} & \mathrm{P} 2 \mathrm{R} 2 & \mathrm{P} 2 \mathrm{R} 3 \\ \text { Number of amino acids } & 77 & 77 \\ \text { Molecular weight (Da) } & 7939.89 & 7919.9 \\ \text { Theoretical pl } & 11.14 & 11.14 \\ \text { Extinction coefficient at } 205 \mathrm{~nm} & 225,230 \mathrm{M}^{-1} \mathrm{~cm}^{-1} & 235,710 \mathrm{M}^{-1} \mathrm{~cm}^{-1}\end{array}$

These values have been obtained using the ExPAsy protparam software ${ }^{201}$.The molar absorptivity at $205 \mathrm{~nm}$ has been calculated using the method presented by Anthis and Clore $^{144}$

\section{$9.3 \mathrm{~K} 32$ construct parameters}

Table 9.5 K32 FASTA sequence.

\begin{tabular}{|c|c|c|c|c|}
\hline 10 & 20 & 30 & 40 & 50 \\
\hline MSSPGSPGTP & GSRSRTPSLP & TPPTREPKKV & AVVRTPPKSP & SSAKSRLQTA \\
\hline 60 & 70 & 80 & 90 & 100 \\
\hline PVPMPDLKNV & KSKIGSTENL & KHQPGGGKVQ & IINKKLDLSN & VQSKCGSKDN \\
\hline 110 & 120 & 130 & 140 & 150 \\
\hline I KHVPGGGSV & QIVYKPVDLS & KVTSKCGSLG & NI HHKP GGGQ & VEVKSEKLDF \\
\hline 160 & 170 & 180 & 190 & \\
\hline KDRVQSKI GS & LDNITHVPGG & GNKKIETHKL & TFRENAKAKT & DHGAEIVY \\
\hline
\end{tabular}

\subsubsection{K32 physical and chemical parameters}

$\begin{array}{ll}\text { Number of amino acids } & 198 \\ \text { Molecular weight (Da) } & 21030.11 \\ \text { Theoretical pl } & 10.09\end{array}$

\subsection{STD NMR of tau peptides binding to soluble tubulin}

Here are reported additional STD NMR experiments performed on tau peptides, shown in Figure 9.1. The affinity of the Tau(P2) peptide has been reported and discussed in the previous section 5.9. The peptides Tau(P2)short, Tau(P2-R3) and Tau(R3)short have been used to study the contribution of different tau domains in the interaction with tubulin. Their sequences are indicated below: 
Tau(P2)short. (K224-S238) Ac-KKVAVVRTPPKSPSS-NH2

Tau(P2-R3) (K224-V313) Ac-KKVAVVRTPPKSPSSVPGGGSVQIVYKPV-NH2

Tau(R3)short. (V300-V313) Ac-VPGGGSVQIVYKPV-NH2

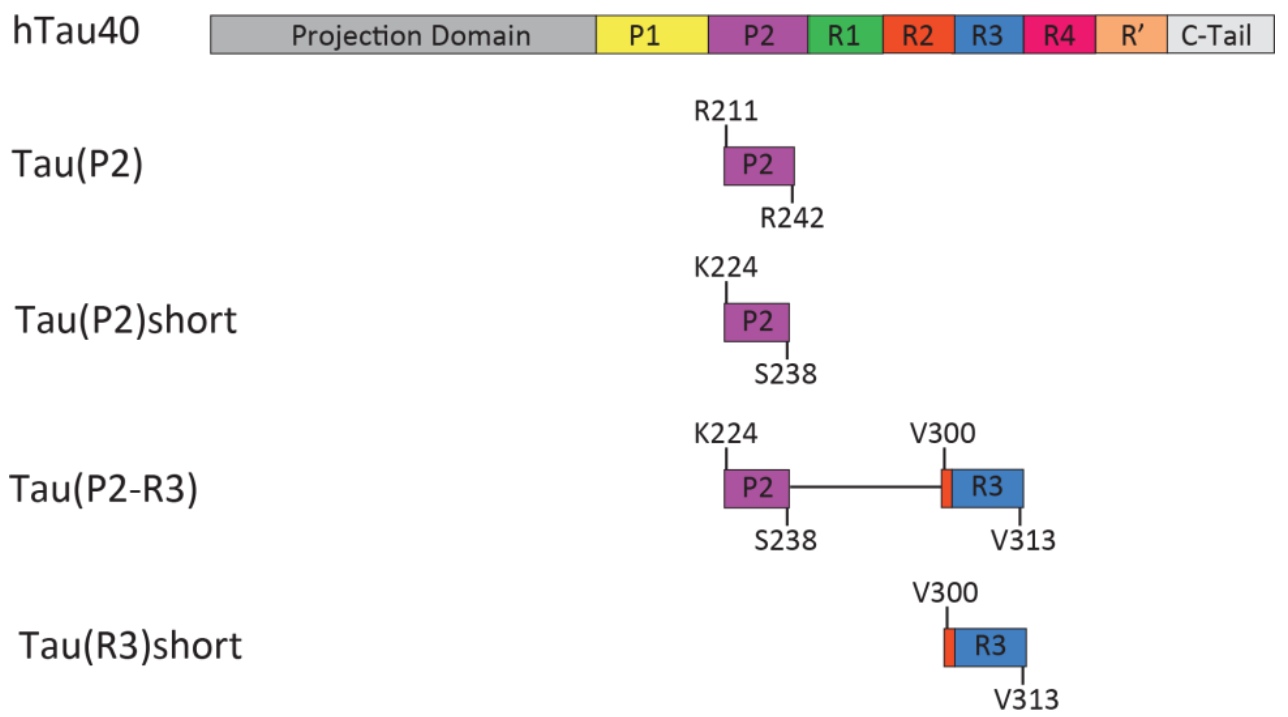

Figure 9.1. Tau peptides used for additional STD NMR experiments. Schematic representation of the domain organization of the four peptides, which combine residues from the P2 and the R3 domain. For comparison, the domain organization of htau40 and Tau(P2) is represented on top.

${ }^{1} \mathrm{H}$ 1D STD-NMR experiments have been recorded at $5{ }^{\circ} \mathrm{C}$ for each peptide using a fixed tubulin concentration of $5 \mu \mathrm{M}$. For the general parameter settings of the STD experiment see section 2.10.6.1. The quantification of the binding affinities has been performed by integration ofselected isolated peaks in the amide and methyl region of the ${ }^{1} \mathrm{H} 1 \mathrm{D}$ spectra, similar to Tau(P2) (section 5.9). Integrals of the cross peaks assigned to T231 (0.97 ppm) and A227 (1.06 ppm) were taken into consideration for the binding affinity of the P2 domain. For the R3 domain, the integral of the unique $\mathrm{Y} 310(6.71 \mathrm{ppm})$ in the sequence was selected (Figure 9.2). 


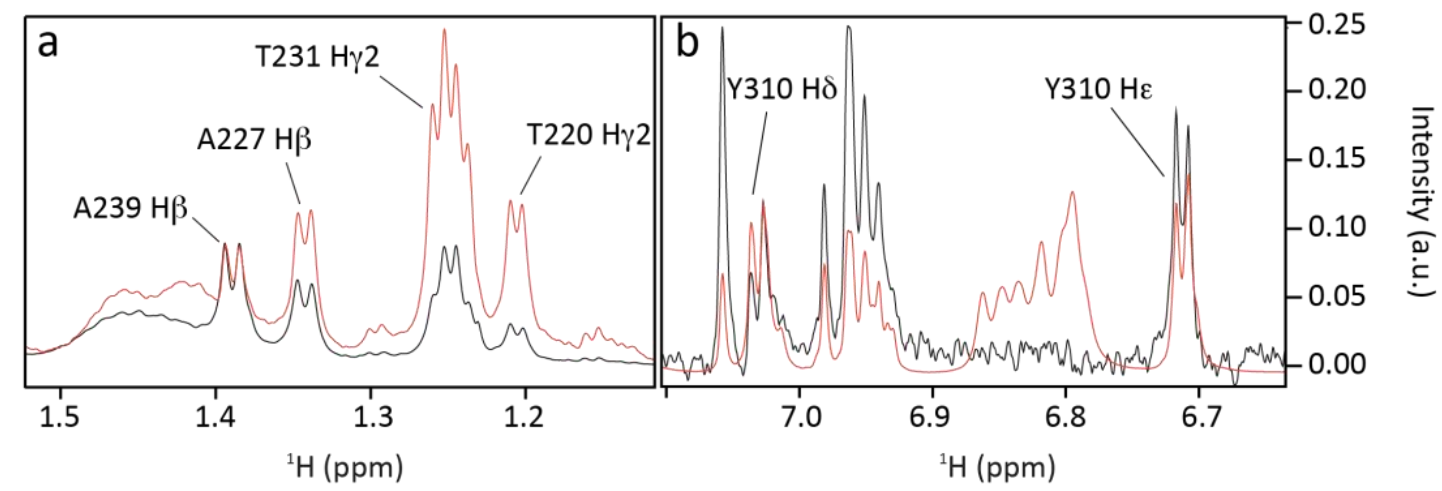

Figure 9.2.Selection of peaks in the1H 1D STD-NMR spectra for the analysis of the binding affinites. Superposition of the methyl region (a) and amide region (b) of the ${ }^{1} \mathrm{H} 1 \mathrm{D}$ spectrum (red) and ${ }^{1} \mathrm{H}$ 1D STD spectrum (black) of Tau(P2-R3) to indicate the signals used for the analysis of the spectra.

As already reported in section 5.9, the P2 domain binds weakly to soluble tubulin. The Tau(P2)short, which comprises the residues K224-S238 flanking the hydrophobic ${ }^{225} \mathrm{KVAVVRT}^{231}$ stretch, also displayeda similar weak binding. The $\mathrm{K}_{\mathrm{D}}$ values calculated for the A227 and T231 resonances in this peptide were $\sim 1 \mathrm{mM}$ and $~ 1.5 \mathrm{mM}$ (Table 9.6). In combination with the R3 domain, i.e. in Tau(P2-R3), the affinity of the P2 domain remained weak, displaying a $K_{D}$ value of $\sim 2-3 \mathrm{mM}$. This suggested that the P2 domain interacted weakly with soluble tubulin, independently from the presence of an element of the pseudo-repeat region. In contrast, the affinity of Y310 in the R3 domain was 363.3 $\pm 135.7 \mu \mathrm{M}$ and $\sim 268.8 \pm 80.4 \mu \mathrm{M}$ for Tau(R3)short and for Tau(P2-R3), respectively (Table 9.2 and Figure 9.3). This observation was consistent with what was previously reported by biochemical analyses on different tau constructs, that the repeat regions can bind tubulin in a modular way, but the presence of the P2 domain enhances their affinity ${ }^{50}$. In tubilin polymerization assays,they showed to have small effect in absence of the proline-rich region as well. This suggests that a combination of at least one pseudo-repeat domain and the proline-rich region is necessary for efficient microtubule formation ${ }^{50,52,117}$. The $K_{D}$ values reported for the $\mathrm{R} 3$ domain, alone or in combination with the P2 domain, were weaker when compared to binding affinities reported for tau constructs, e.g. K19 and K18, measured by co-sedimentation assays ${ }^{50}$. Nevertheless, Kadavath and co-workers studied the binding of the $\mathrm{R}^{\prime}$ domain of htau40 using peptides of similar length to the peptides used in this study, i.e. 15-30 residues. The $K_{D}$ values reported for F378 and Y394 in the R' 
domains were of $381.7 \mu \mathrm{M}$ and $356.3 \mu \mathrm{M}$ respectively and were comparable to those reported for the R3 domain in Tau(R3)short and Tau(P2-R3) ${ }^{108}$.

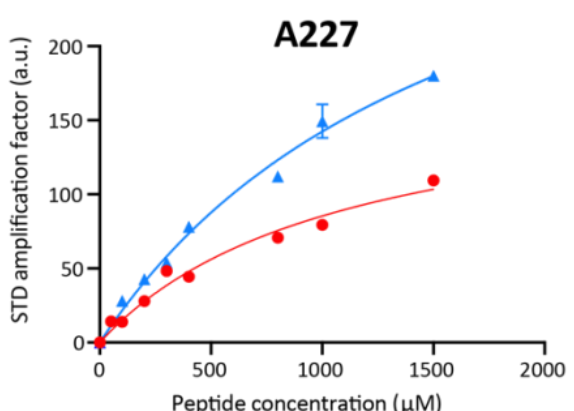

T231

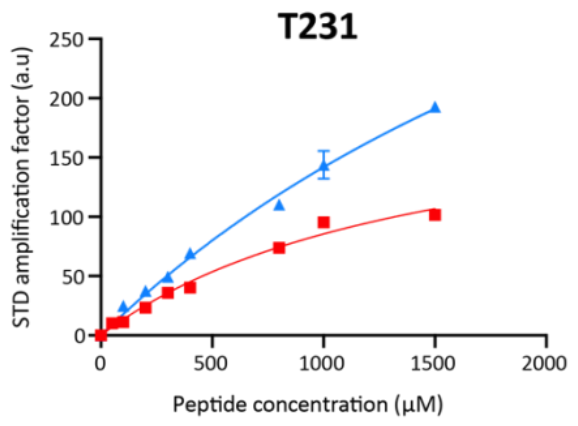

Figure 9.3. Binding affinities of selected aminoacid residues in Tau(P2)short and Tau(P2-R3).Quantification of saturation transfer as a function of the Tau(P2)short/Tau(P2-R3) concentration. Binding curves are shown for A227 and T231, located in the proline-rich region, and Y310, located in the R3 domain. The $K_{D}$ values were calculated by titrating increasing concentration of each peptide to a fixed concentration of tubulin ( 5 $\mu \mathrm{M}$ ). Measurements were performed at $5^{\circ} \mathrm{C}$ in non- polymerizing BRB80 Buffer (80 mM Pipes, pH 6.8, 1 $\mathrm{mM} \mathrm{MgSO}_{4}, 1 \mathrm{mM}$ EDTA, $1 \mathrm{mM}$ DTT). Error bars were calculated on the basis of the spectral signal-to-noise ratio.

Table 9.6 KD values reported for selected residues located in two different domains of tau.

$$
\text { Y310(R3) }
$$$$
\text { A227(PRR) }
$$

Tau(P2)short

$$
\mathrm{X}
$$

$1095.0 \pm 306.4 \mu \mathrm{M}$

$1477.3 \pm 382.3 \mu \mathrm{M}$

$\mathrm{Tau}(\mathrm{P} 2-\mathrm{R} 3)$

$268.8 \pm 80.4 \mu \mathrm{M}$

$1661.0 \pm 368.9 \mu \mathrm{M}$

$3339.2 \pm 867.6 \mu \mathrm{M}$

Tau(R3)short

$$
363.3 \pm 135.7 \mu \mathrm{M}
$$

$x$

X 


\section{5 ${ }^{1} \mathrm{H}-{ }^{15} \mathrm{~N}$ HSQC pulse program}

;hsqc15N.new

;D. Lee. Nov. 2002

;15N-1H HSQC correlations without water saturation ;The delay for 3-9-19 watergate ( $d 5$ ) should be matched ; with $1 / d$; d=distance of next null point (in $\mathrm{Hz}$ ).

;S. Mori et al. JMR B108. 94-98 (1995)

;pl1 : power for $1 \mathrm{H}$

;pl2 : power for $13 \mathrm{C}$

;pl3 : power for $15 \mathrm{~N}$

;pl13 : power for $15 \mathrm{~N}$ waltz16 decoupling

;p1 : 90 degree hard pulse $1 \mathrm{H}$

;p3 : 90 degree hard pulse $13 \mathrm{C}$

;p4 : 180 degree hard $13 C$ pulse (225d for $5 / 600$ )

;p5 : 90 degree hard pulse $15 \mathrm{~N}$

;pcpd3 : 90 deg cpd-pulse15N(waltz16.160u)

;d1 : relaxation delay

;d2 : INEPT delay ( $2.7 \mathrm{~m})$

;d5 : delay for $3-9-19=1 /(\mathrm{Hz}$ between nulls)

;in0 : $1 /(2 \mathrm{SW})(\mathrm{Hz})$

;p21 : 500u (Gradient in first INEPT)

;p22 : 500u (Gradient for z-filter)

;p23 : 1m (Gradient for second INEPT)

;gpz1 : 19\%

;gpz2 : 30\%

;gpz3 : 65\%

; CLASS=HighRes Incl

;\$COMMENT=

;\$ld: Avance3.incl.v 1.6.2.1 2007/09/14 16:17:35 ber Exp \$

define delay INEPT_W

define delay INEPT_D

"p2=2*p1"

"p6=2*p5"

"in $0=$ inf $1 / 2$ "

"d0=in0/2-p5*2/3.14159"

"d3=d5/2-p5"

"INEPT_D=d2-p21-210u"

"INEPT_W=d2-(p23+210u+p1*2.3846+d5*2.5)" 


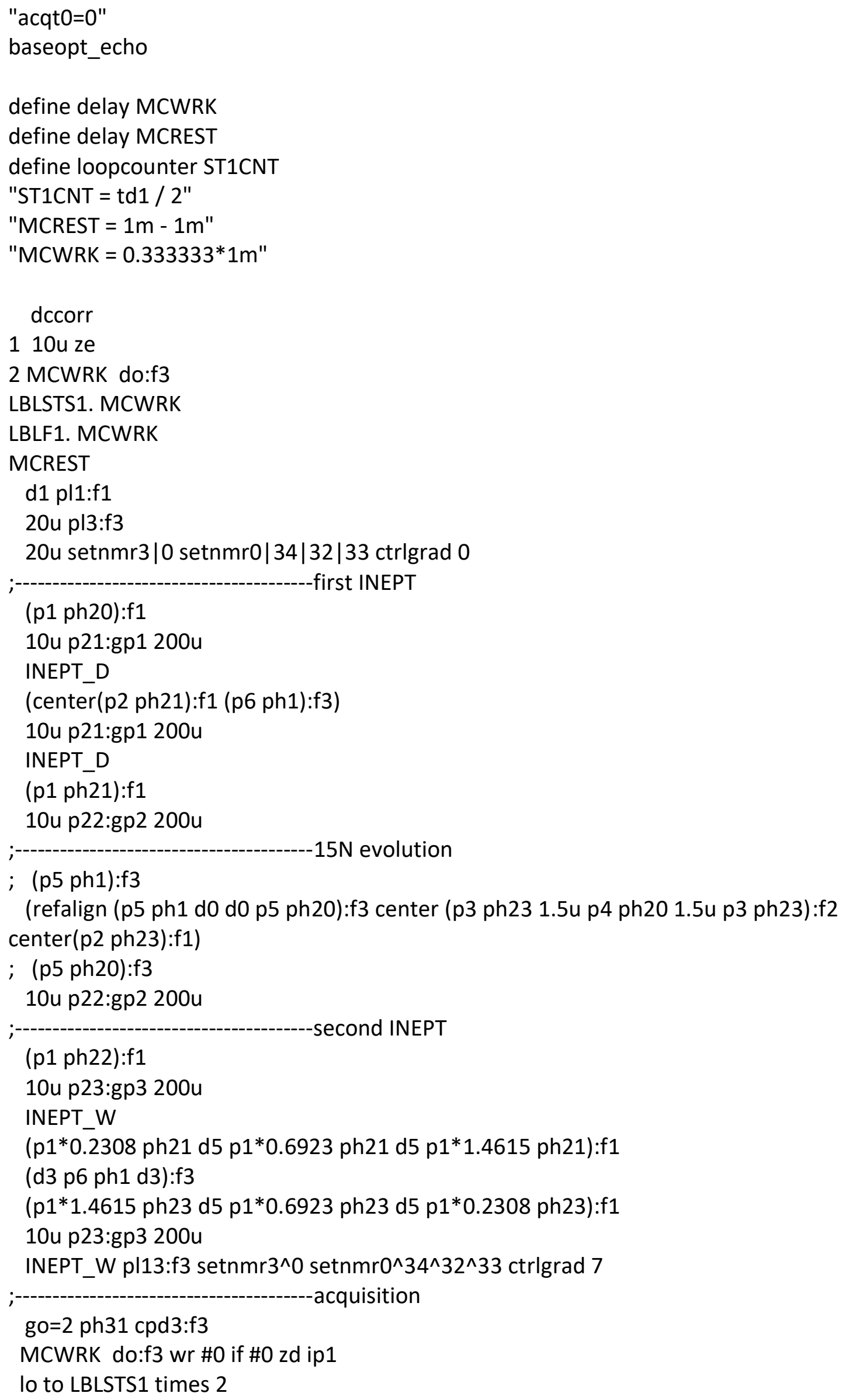




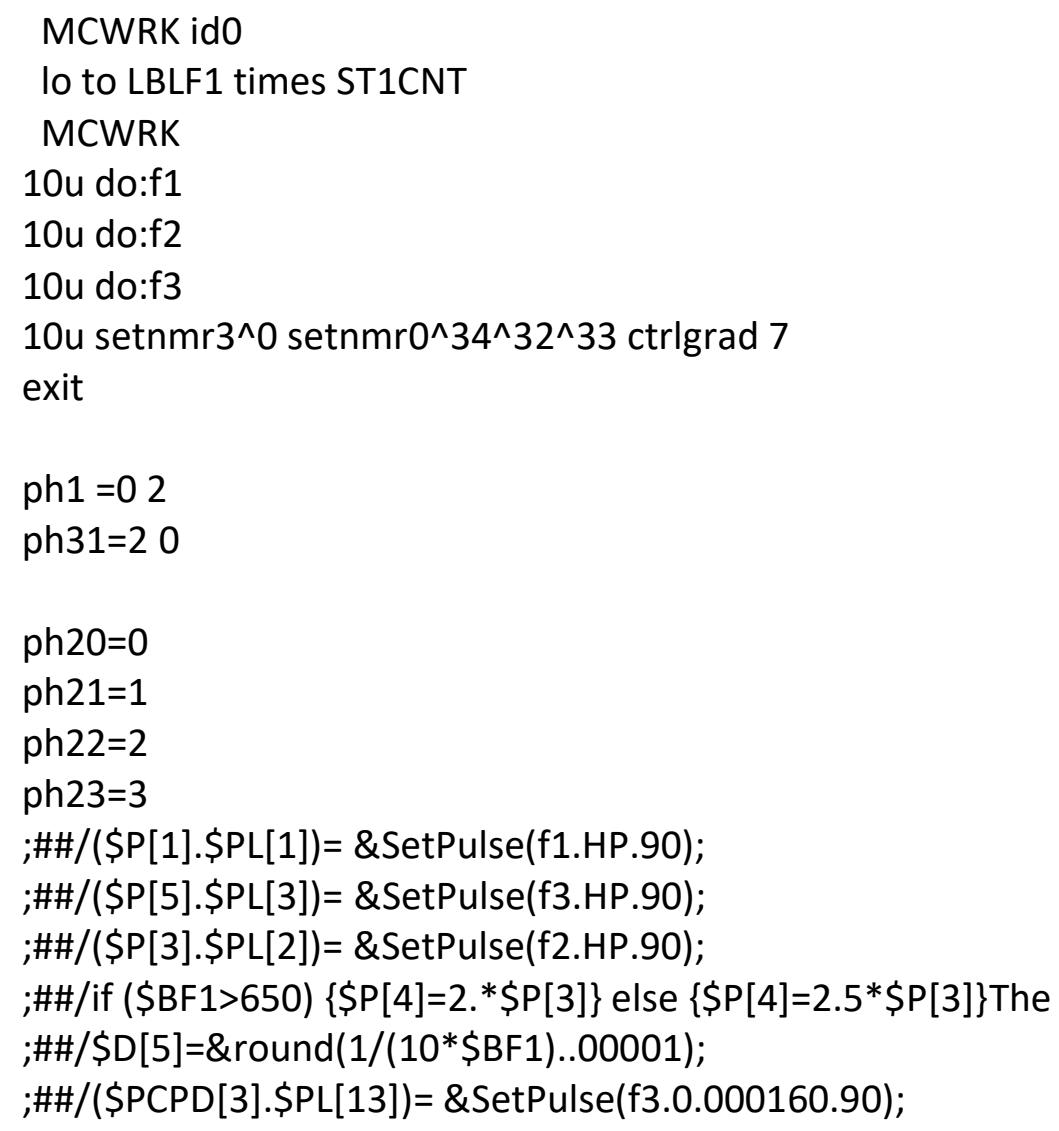

\section{9. ${ }^{1} \mathrm{H}$ 1D STD NMR experiment pulse program}

;stddiffgp19.2

;avance-version (13/11/08)

;pseudo 2D sequence

; for saturation transfer difference

; with shaped pulse train for saturation on $\mathrm{f} 2$ channel

;alternating between on and off resonance

; to be defined by fq2list

; with spoil sequence to destroy unwanted magnetization ;water suppression using 3-9-19 pulse sequence with gradients ;(use parameterset STDDIFFGP19.2)

;M. Mayer \& B. Meyer. Angew. Chem. Int. Ed. 38. 1784-1788 (1999)

;M. Mayer \& B. Meyer. Angew. Chem. 111. 1902-1906 (1999)

;M. Piotto. V. Saudek \& V. Sklenar. J. Biomol. NMR 2. 661 - 666 (1992)

;V. Sklenar. M. Piotto. R. Leppik \& V. Saudek. J. Magn. Reson..

; Series A 102. 241 -245 (1993)

; CLASS=HighRes

;\$DIM=2D

;\$TYPE= 
;\$SUBTYPE= ;\$COMMENT=

\#include <Avance.incl $>$

\#include <Grad.incl $>$

\#include <Delay.incl $>$

define list $<$ frequency $>$ stdlist $=<\$$ FQ2LIST $>$

"d11=30m"

"I5=d20/p42"

"d31=p42*15"

"DELTA1=d1-d31"

"TAU=p1*2/3.1416-(p0-p27)*0.231-de+46u"

"acqt $0=0 "$

baseopt_echo

1 ze

$3 \mathrm{~m}$ stdlist:f2 st0

$26 \mathrm{~m}$

$36 \mathrm{~m}$

$4 \mathrm{~d} 11$

$6 \mathrm{~m}$

5 50u UNBLKGRAD

4u pl10:f1

(p17 ph2)

(p17*2 ph3)

$4 \mathrm{u}$

p30:gp1

10m pl1:f1

4u BLKGRAD

DELTA1

6 (p42:sp9 ph4):f2

$4 \mathrm{u}$

lo to 6 times 15

p1 ph1

50u UNBLKGRAD

p16:gp2 
d16 pl18:f1

p27*0.231 ph5

d19*2

p27*0.692 ph5

d19*2

p27*1.462 ph5

d19*2

p27*1.462 ph 6

d $19 * 2$

p27*0.692 ph 6

d19*2

p0*0.231 ph6

TAU

p16:gp2

d16

4u BLKGRAD

goscnp ph31

$3 m$ stdlist.inc

$3 \mathrm{~m}$ stdlist: $\mathrm{f} 2 \mathrm{st}$

lo to 3 times $\mathrm{nbl}$

3m ipp1 ipp5 ipp6 ipp31

$3 \mathrm{~m}$ stdlist.res

lo to 4 times ns

d11 wr \#0

$3 \mathrm{~m}$ rppall

$3 \mathrm{~m} z d$

lo to 5 times 14

exit

ph1 $1=02$

ph2 $=0$

ph3 $=1$

ph4 $=0$

ph5=0 01112233

ph6=2 23300011

ph31=0 220

;pl1 : f1 channel - power level for pulse (default)

;pl2 : f2 channel - power level for pulse (default)

$[120 \mathrm{~dB}]$

;pl10: f1 channel - power level for TOCSY-spinlock

;pl18: f1 channel - power level for 3-9-19-pulse (watergate)

;sp9 : $\mathrm{f} 2$ channel - shaped pulse for saturation [40-60 dB]

;p0 : f1 channel - 90 degree pulse at pl18

; $\quad$ use for fine adjustment

;p1 : f1 channel - 90 degree high power pulse 
;p16: homospoil/gradient pulse

;p17: f1 channel - trim pulse

[2.5 msec]

;p27: $f 1$ channel - 90 degree pulse at pl18

;p30: gradient pulse

[3 msec]

;p42: $\mathrm{f} 2$ channel - shaped pulse for saturation [50 msec]

;d1 : relaxation delay; $1-5 * \mathrm{~T} 1$

;d11: delay for disk $1 / 0$

[30 msec]

;d16: delay for homospoil/gradient recovery

;d19: delay for binomial water suppression

; $\quad$ d19 $=(1 /(2 * d)) \cdot d=$ distance of next null (in $\mathrm{Hz}$ )

;d20: saturation time

;d31: saturation time as executed

;14: 14 = number of averages = (total number of scans) / NS

;15: loop for saturation: $\mathrm{p} 42 * 15=$ saturation time

;ns: $8 * \mathrm{n}$

;ds: 4

;td1: number of experiments

;NBL: NBL = number of irradiation frequencies

;define FQ2LIST (irradiation frequencies)

; $\quad$ (list has to be stored in "/u/exp/stan/nmr/lists/f1")

;use gradient ratio: gp 1 : gp 2

; $40: 30$

;for z-only gradients:

;gpz1: $40 \%$

;gpz2: $30 \%$

; use gradient files:

;gpnam1: SMSQ10.100

;gpnam2: SMSQ10.100

;this pulse program produces a ser-file (PARMOD = 2D)

; The STD experiment is protected by international patents owned by:

;Alepharma Licensing. Raamfeld 67. 22397 Hamburg. Germany.

;For commercial use (direct or indirect) please contact the company for

;licensing information at:

;E-mail: info@alepharma-licensing.com.

;Fax: +49 4060847812.

;Tel: +49 1701685158 or +49 1712788867 .

;\$ld: stddiffgp19.2.v 1.9.2.1 2014/01/17 15:19:20 ber Exp \$ 


\subsection{NMR diffusion PGSTE-WATERGATE pulse program}

;PGSTE WATERGATE 2D

;set up by sebi (March 2010)

;modified by nare

;2D sequence for diffusion measurement with

;water suppression using watergate W5 pulse sequence with gradients

;G. Zheng. T. Stait-Gardner. P.G. Anil Kumar. A.M. Torres and W.S. Price ;J. Magn. Reson. 191 (2008). pp. 159163

;d1: 7 to 10 seconds (avoid sample heating)

;p30: little delta

; delta2 is fixed at $2 \mathrm{~ms}$

;gpz5: around 20\%

;gpz6: incremented from $25-95 \%$

;gpz7: $6.66 \%$

;d20: specifies big Delta (around 100ms)

;d19: delay for binomial water suppression

; $\mathrm{d} 19=$ distance of next null (200us $\sim 5000 \mathrm{~Hz}$ )

;ns $=4 * n$

; use "Difftrap" as gradient pulses

;type "difftrap" before the start of the experiment

;use AU-program dosy to calculate gradient ramp-file Difframp

;\$CLASS=HighRes Incl

;\$COMMENT=

\section{8 ssNMR PDSD pulse program}

;hxinept2D

;2D heteronuclear shift correlation ; with refocussing of chem. shifts ;phase sensitive

;A. Bax \& G.A. Morris. J. Magn. Reson. 42. 501 (1981)

;sewe 31.01.2013

;TS 3.X Version

;pl1 : f1 channel - power level for pulse

;pl2 : =0W. not used

;pl12: f2 channel - power level for Decoupling and p3

;p1 : f1 channel - 90 degree high power pulse

;p2 : f1 channel - 180 degree high power pulse 
;p3 : f2 channel - 90 degree high power pulse

;p4 : f2 channel - 180 degree high power pulse

;d0 : incremented delay (2D)

;d1 : relaxation delay; $1-5 * \mathrm{~T} 1$

;d3 : 1/(6J(XH)) XH. XH2. XH3 positive

; $1 /(4 \mathrm{~J}(\mathrm{XH})) \times \mathrm{XH}$ only

; $1 /(3 \mathrm{~J}(\mathrm{XH})) \mathrm{XH} . \mathrm{XH} 3$ positive. $\mathrm{XH} 2$ negative

;d4 : 1/(4J(XH))

;cnst2: $=\mathrm{J}(\mathrm{XH})$

;cnst11: $=6 \mathrm{XH}$. XH2. XH3 positive

; $4 \mathrm{XH}$ only

; $3 \mathrm{XH}$. $\mathrm{XH} 3$ positive. $\mathrm{XH} 2$ negative

;inf1: $1 / \mathrm{SW}(\mathrm{H})=2 * \mathrm{DW}(\mathrm{H})$

;in0: $1 /(2 * S W(H))=D W(H)$

;nd0: 2

;ns: $2 * n$

;td1: number of experiments

;FnMODE: States-TPPI. TPPI. States or QSEQ

;cpd2: decoupling according to sequence defined by cpdprg2

;pcpd2: f2 channel - 180deg on $1 \mathrm{H}$

;cpdprg2: decoupling sequence. e.g spinal64_12

;CLASS=Solids

;\$DIM=2D

;\$TYPE=Heteronucelar correlation

;\$SUBTYPE=

;\$COMMENT=use hxinept1Dopt for setup

\# 1 "/opt/topspin3.5pl6/exp/stan/nmr/lists/pp/Avance.incl" 1 ;Avance3.incl

; for AV III

;avance-version (13/06/17)

;\$CLASS=HighRes Incl ;\$COMMENT=

\# 169 "/opt/topspin3.5pl6/exp/stan/nmr/lists/pp/Avance.incl"

;\$ld: Avance3.incl.v 1.10.2.2 2013/08/30 09:43:33 ber Exp \$

\# 1 "/opt/topspin3.5pl6/exp/stan/nmr/lists/pp/Delay.incl" 1

;Delay.incl - include file for commonly used delays

;version (13/08/07)

;\$CLASS=HighRes Incl

;\$COMMENT= 
\# 9 "/opt/topspin3.5pl6/exp/stan/nmr/lists/pp/Delay.incl" ;general delays

define delay DELTA

define delay DELTA1

define delay DELTA2

define delay DELTA3

define delay DELTA4

define delay DELTA5

define delay DELTA6

define delay DELTA7

define delay DELTA8

define delay DELTA9

define delay DELTA10

define delay DELTA11

define delay DELTA12

define delay DELTA13

define delay DELTA14

define delay DELTA15

define delay DELTA16

define delay TAU

define delay TAU1

define delay TAU2

define delay TAU3

define delay TAU4

define delay TAU5

define delay TAU6

define delay TAU7

define delay TAU8

define delay TAU9

\# 40 "/opt/topspin3.5pl6/exp/stan/nmr/lists/pp/Delay.incl"

define delay INCR1

define delay INCR2

define delay INCR3

define delay INCR4

define delay INCR5

define delay INCR6

;delays for centering pulses

\# 50 "/opt/topspin3.5pl6/exp/stan/nmr/lists/pp/Delay.incl" define delay CEN_HN1

define delay CEN_HN2

define delay CEN_HN3

define delay CEN_HC1 
define delay CEN_HC2

define delay CEN_HC3

define delay CEN_HC4

define delay CEN_HP1

define delay CEN_HP2

define delay CEN_CN1

define delay CEN_CN2

define delay CEN_CN3

define delay CEN_CN4

define delay CEN_CP1

define delay CEN_CP2

;loop counters

\# 69 "/opt/topspin3.5pl6/exp/stan/nmr/lists/pp/Delay.incl"

define loopcounter COUNTER

define loopcounter SCALEF

define loopcounter FACTOR1

define loopcounter FACTOR2

define loopcounter FACTOR3

define loopcounter FACTOR4

define loopcounter FACTOR5

define loopcounter FACTOR6

\# 80 "/opt/topspin3.5pl6/exp/stan/nmr/lists/pp/Delay.incl"

;\$ld: Delay.incl.v 1.14.2.1 2013/08/30 09:43:33 ber Exp \$

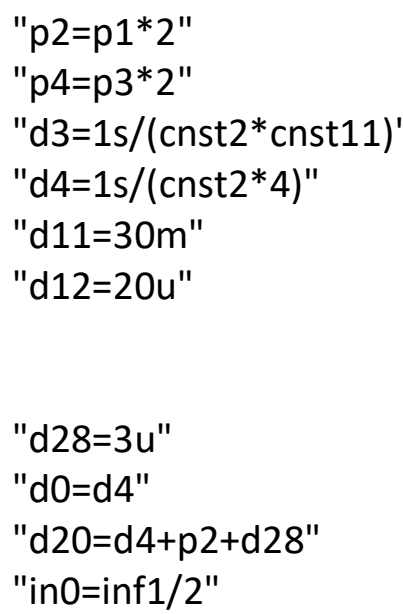

"DELTA=d3+(p1*2/PI)-de-1u"

"FACTOR1 $=d 20 * 10000000 * 2 / \mathrm{td} 1 "$

"in20=FACTOR1/10000000" 
"if ( in20 > in $)$ ) in28=0; $\}$ else $\{$ in28=in0-in20; $\}$ "

"if ( in20 > in0 ) \{ in20=in0; $\}$ "

"acqt0 $=0 "$

baseopt_echo

; dimension 2D; AQ_mode (F1) States-TPPI

define delay MCWRK

define delay MCREST

define delay d0orig

"d0orig=d0"

define delay d20orig

"d20orig=d20"

define delay d28orig

"d28orig=d28"

define loopcounter t1loop

"t1loop=0"

define loopcounter ph1loop

"ph1loop=0"

define loopcounter ST1CNT

"ST1CNT = td1 / 2"

"MCREST $=10 \mathrm{~m}-10 \mathrm{~m} "$

"MCWRK $=0.200000 * 10 \mathrm{~m} "$

dccorr

1 ze

LBLAV. MCWRK

"d0=d0orig + t1loop * in $"$

"d20=d20orig - t1loop * in20 "

"d28=d28orig + t1loop * in28 "

"phval3 = (ph1loop \% 4) * 90"

MCWRK ip3 + phval3

2 MCWRK * 2 do:f2

LBLF1. MCWRK * 2

LBLST1. MCWRK

MCREST

$3 \mathrm{~d} 1$

(p3 pl2 ph1):f2

d0

(p2 pl1 ph4):f1

d28

(p4 pl2 ph2):f2

d20

(p3 pl2 ph3):f2

(p1 pl1 ph5):f1

d3

(center (p4 pl2 ph2):f2 (p2 pl1 ph6):f1 ) 
DELTA

1u pl12:f2

go=2 ph31 cpds2:f2

MCWRK do:f2 wr \#0 if \#0 zd

"ph1loop+=1"

"d0=d0orig + t1loop * in $"$

"d20=d20orig - t1loop * in20 "

"d28=d28orig + t1loop * in28 "

"phval3 = (ph1loop \% 4) * 90"

MCWRK ip3 + phval3

lo to LBLF1 times 2

MCWRK

"t1loop+=1"

"d0=d0orig + t1loop * in0 "

"d20=d20orig - t1loop * in20 "

"d28=d28orig + t1loop * in28 "

"phval3 = (ph1loop \% 4) * 90"

MCWRK ip3 + phval3

lo to LBLST1 times ST1CNT

MCWRK

"t1loop=0"

"ph1loop=0"

MCWRK rf \#0

lo to LBLAV times tdav

exit

ph1 $=0$

ph2=0 022

ph3 $=13$

ph4=0 022

ph5=0000111122223333

ph6=0 0221133

ph31=0202131320203131

;\$ld: hxinept2D.v 1.1.4.1 2014/02/11 09:04:53 ber Exp \$

\subsection{INEPT-based ${ }^{1} \mathrm{H}^{13} \mathrm{C}$ correlation pulse program}

;2D heteronuclear shift correlation ; with refocussing of chem. shifts ;phase sensitive

;A. Bax \& G.A. Morris. J. Magn. Reson. 42. 501 (1981) 
;sewe 31.01.2013

;TS 3.X Version

;pl1 : f1 channel - power level for pulse

;pl2 : =0W. not used

;pl12: f2 channel - power level for Decoupling and $p 3$

;p1 : f1 channel - 90 degree high power pulse

;p2 : f1 channel - 180 degree high power pulse

;p3 : f2 channel - 90 degree high power pulse

;p4 : f2 channel - 180 degree high power pulse

;d0 : incremented delay (2D)

;d1 : relaxation delay; $1-5 * \mathrm{~T} 1$

;d3 : 1/(6J(XH)) XH. XH2. XH3 positive

; $1 /(4 \mathrm{~J}(\mathrm{XH})) \mathrm{XH}$ only

; $1 /(3 \mathrm{~J}(\mathrm{XH})) \mathrm{XH}$. XH3 positive. $\mathrm{XH} 2$ negative

;d4 : 1/(4J(XH))

;cnst2: $=\mathrm{J}(\mathrm{XH})$

;cnst11: $=6 \mathrm{XH}$. XH2. XH3 positive

; $4 \mathrm{XH}$ only

; $3 \mathrm{XH} . \mathrm{XH} 3$ positive. $\mathrm{XH} 2$ negative

;inf1: $1 / S W(H)=2 * D W(H)$

;in0: $1 /(2 * S W(H))=D W(H)$

;nd0: 2

;ns: $2 * n$

;td1: number of experiments

;FnMODE: States-TPPI. TPPI. States or QSEQ

;cpd2: decoupling according to sequence defined by cpdprg2

;pcpd2: f2 channel - 180deg on $1 \mathrm{H}$

;cpdprg2: decoupling sequence. e.g spinal64_12

; $\$$ CLASS=Solids

;\$DIM=2D

;\$TYPE=Heteronucelar correlation

;\$SUBTYPE=

; $\$$ COMMENT=use hxinept1Dopt for setup

\# 1 "/opt/topspin3.5pl6/exp/stan/nmr/lists/pp/Avance.incl" 1

;Avance3.incl

; for AV III

;avance-version (13/06/17)

;\$CLASS=HighRes Incl

;\$COMMENT= 
\# 169 "/opt/topspin3.5pl6/exp/stan/nmr/lists/pp/Avance.incl" ;\$Id: Avance3.incl.v 1.10.2.2 2013/08/30 09:43:33 ber Exp \$

\# 1 "/opt/topspin3.5pl6/exp/stan/nmr/lists/pp/Delay.incl" 1 ;Delay.incl - include file for commonly used delays

;version (13/08/07)

;CLASS=HighRes Incl ;\$COMMENT=

\# 9 "/opt/topspin3.5pl6/exp/stan/nmr/lists/pp/Delay.incl" ;general delays

define delay DELTA define delay DELTA1 define delay DELTA2 define delay DELTA3 define delay DELTA4 define delay DELTA5 define delay DELTA6 define delay DELTA7 define delay DELTA8 define delay DELTA9 define delay DELTA10 define delay DELTA11 define delay DELTA12 define delay DELTA13 define delay DELTA14 define delay DELTA15 define delay DELTA16

define delay TAU define delay TAU1 define delay TAU2 define delay TAU3 define delay TAU4 define delay TAU5 define delay TAU6 define delay TAU7 define delay TAU8 define delay TAU9 \# 40 "/opt/topspin3.5pl6/exp/stan/nmr/lists/pp/Delay.incl" define delay INCR1 define delay INCR2 define delay INCR3 define delay INCR4 
define delay INCR5

define delay INCR6

;delays for centering pulses

\# 50 "/opt/topspin3.5pl6/exp/stan/nmr/lists/pp/Delay.incl"

define delay CEN_HN1

define delay CEN_HN2

define delay CEN_HN3

define delay CEN_HC1

define delay CEN_HC2

define delay CEN_HC3

define delay CEN_HC4

define delay CEN_HP1

define delay CEN_HP2

define delay CEN_CN1

define delay CEN_CN2

define delay CEN_CN3

define delay CEN_CN4

define delay CEN_CP1

define delay CEN_CP2

;loop counters

\# 69 "/opt/topspin3.5pl6/exp/stan/nmr/lists/pp/Delay.incl" define loopcounter COUNTER

define loopcounter SCALEF

define loopcounter FACTOR1

define loopcounter FACTOR2

define loopcounter FACTOR3

define loopcounter FACTOR4

define loopcounter FACTOR5

define loopcounter FACTOR6

\# 80 "/opt/topspin3.5pl6/exp/stan/nmr/lists/pp/Delay.incl"

;\$ld: Delay.incl.v 1.14.2.1 2013/08/30 09:43:33 ber Exp \$

"p2=p1*2"

"p4=p3*2"

"d3=1s/(cnst2* cnst11)"

"d4=1s/(cnst2*4)"

"d11=30m"

"d12=20u"

"d28=3u"

"d0=d4" 
"d20=d4+p2+d28"

"in0=inf1/2"

"DELTA=d3+(p1*2/PI)-de-1u"

"FACTOR $1=\mathrm{d} 20 * 10000000 * 2 / \mathrm{td} 1 "$

"in20=FACTOR1/10000000"

"if ( in20 > in0 ) $\{$ in28=0; $\}$ else $\{$ in28=in0-in20; $\} "$

"if ( in $20>$ in 0 ) $\{$ in20=in 0 ; $\} "$

"acqt $0=0$ "

baseopt_echo

; dimension 2D; AQ_mode (F1) States-TPPI

define delay MCWRK

define delay MCREST

define delay d0orig

"d0orig=d0"

define delay d20orig

"d20orig=d20"

define delay d28orig

"d28orig=d28"

define loopcounter t1loop

"t1loop=0"

define loopcounter ph1loop

"ph1loop=0"

define loopcounter ST1CNT

"ST1CNT = td1 / 2"

"MCREST $=10 \mathrm{~m}-10 \mathrm{~m} "$

"MCWRK $=0.200000 * 10 \mathrm{~m} "$

dccorr

1 ze

LBLAV. MCWRK

"d0=d0orig + t1loop * in0 "

"d20=d20orig - t1loop * in20 "

"d28=d28orig + t1loop * in28 "

"phval3 = (ph1loop \% 4) * 90"

MCWRK ip3 + phval3

2 MCWRK * 2 do:f2

LBLF1. MCWRK * 2

LBLST1. MCWRK

MCREST

$3 \mathrm{~d} 1$

(p3 pl2 ph1):f2

d0 


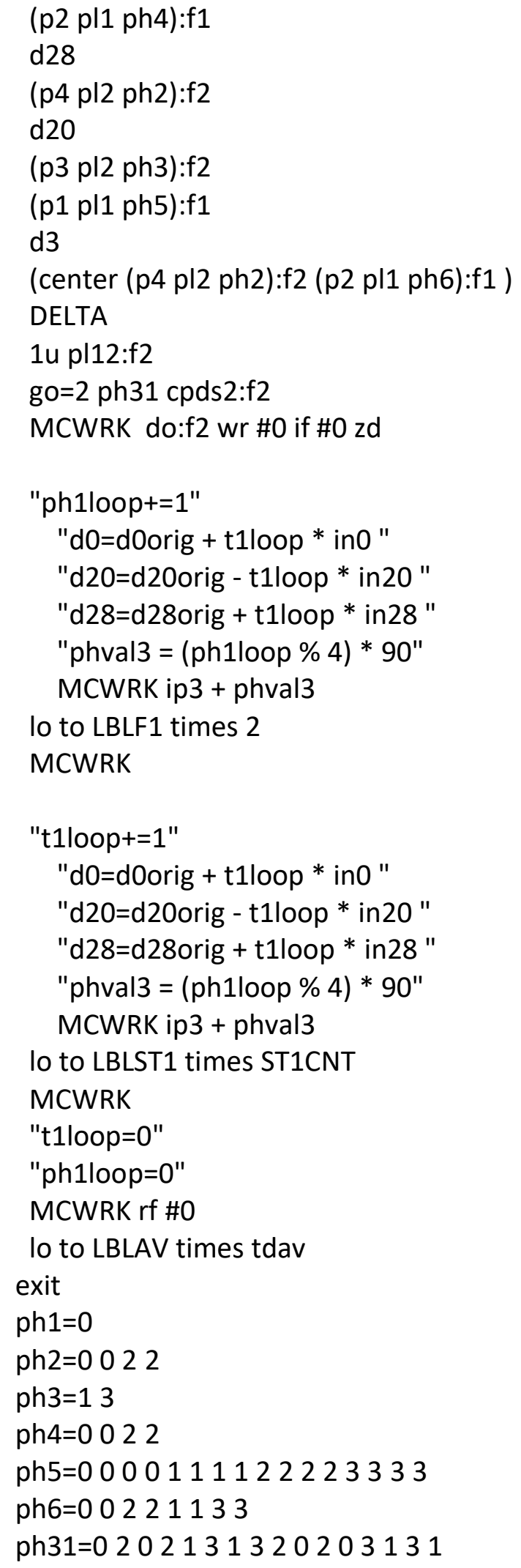

;\$ld: hxinept2D.v 1.1.4.1 2014/02/11 09:04:53 ber Exp \$ 


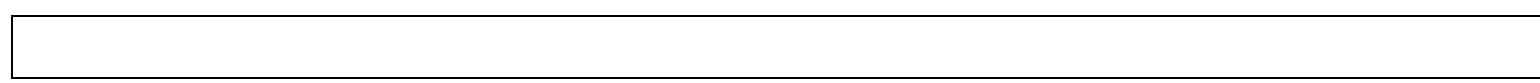

